\title{
Menthols as Chiral Auxiliaries for Asymmetric Cycloadditive Oligomerization: Syntheses and Studies of $\beta$-Proline Hexamers
}

Konstantin V. Kudryavtsev, ${ }^{*}$ Polina M. Ivantcova, Claudia Muhle-Goll, Andrei V. Churakov, Mikhail N. Sokolov, Artem V. Dyuba, Alexander M. Arutyunyan, Judith A.K. Howard, Chia-Chun Yu, Jih-Hwa Guh, Nikolay S. Zefirov, and Stefan Bräse*

Contents

Page Number

General Remarks ...S2

X-ray studies .S3

Table S1 .S5

Figure S1 .56

Figure S2. .56

Figure S3 .S7

Figure S4. ..S7

Figure S5 .S8

Figure S6. .58

Figure S7. ..S9

Figure S8. .S9

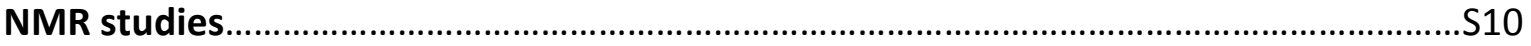

Table S2

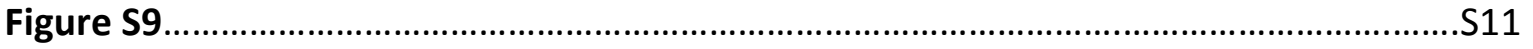

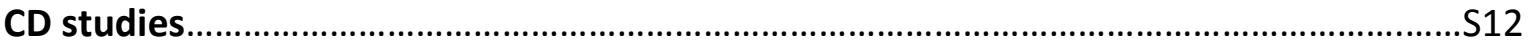

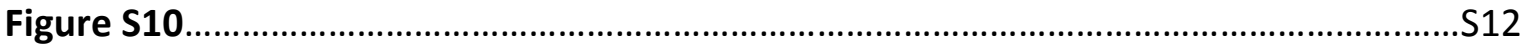

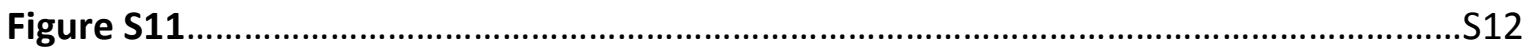

Figure S12

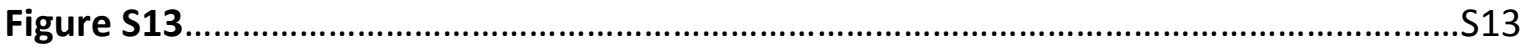

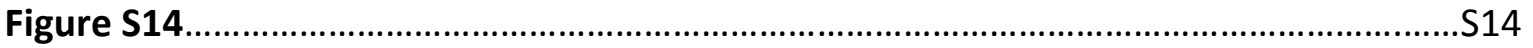

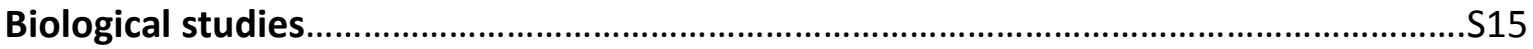

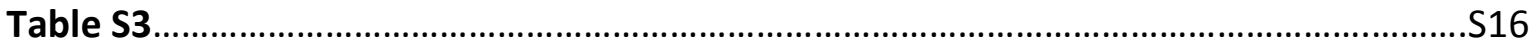

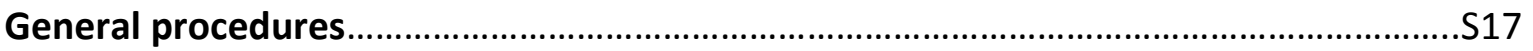

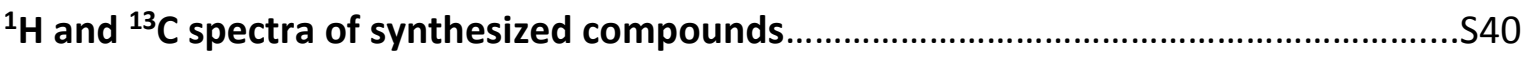




\section{GENERAL REMARKS}

${ }^{1} \mathrm{H}$ NMR spectra were recorded on a Bruker AM-400, Agilent 400 MR, Bruker AVANCE III 600 $\mathrm{MHz}$ spectrometer for solutions in $\mathrm{CDCl}_{3}$ and $\mathrm{DMSO}-\mathrm{d}_{6}$ with tetramethylsilane (TMS) as internal standard. In 2D-NMR experiments tetramer D-4a, pentamer D-5a and hexamer D-6a were studied in DMSO solutions). 2D-1H-1H TOCSY, and NOESY spectra and 2D-1H-13C HSQC spectra were recorded on a Bruker AVANCE III $600 \mathrm{MHz}$ spectrometer at $23{ }^{\circ} \mathrm{C}$. Typical settings were $1024 \mathrm{t}_{1}$ transients with 4 scans per transient for the $2 \mathrm{D}-{ }^{1} \mathrm{H}$ spectra with mixing times of 200 , 400 , and $700 \mathrm{~ms}$ for NOESY spectra, and $60 \mathrm{~ms}$ in the $2 \mathrm{D}-{ }^{1} \mathrm{H}$ TOCSY spectrum. Spectra were analyzed with TOPSPIN 3.0 (Bruker Biospin, Rheinstetten, Germany).

High resolution mass spectra (HRMS) were recorded with a HP-5989 instrument (EI, FAB; Karlsruhe Institute of Technology, Germany) and a Bruker maXis instrument (electrospray ionization (ESI); Zelinsky Institute of Organic Chemistry, Russian Academy of Science, Moscow).

Optical rotations were determined at $589 \mathrm{~nm}$ (sodium D line) by using a Perkin-Elmer-341 MC digital polarimeter and a Jasco DIP-360 polarimeter; $[\alpha]_{D}$-values are given in unit of $10 \mathrm{deg}^{-1} \mathrm{~cm}^{2} \mathrm{~g}^{-1}$.

CD-spectroscopy was performed on Jasco J-810 spectropolarimeter (Jasco, Tokyo, Japan). The spectrum of the solvent was subtracted and spectra analysis software (Jasco, Easton, MD) was used to smoothen the spectra by an adaptive smoothing method.

CD spectra of monomers L-1a and D-1a and temperature dependency of pentamer D-5a were recorded using Chirascan dichrograph («Applied Photophysics», England) in the 185-260 nm range with $1 \mathrm{~nm}$ step, $1 \mathrm{~nm}$ spectral bandwidth, and scan speed of 1s/point, in $0.2 \mathrm{~cm}$ quartz cuvette («Hellma», Germany). Absorption spectra were recorded simultaneously by the same instrument. Temperature in the range of $20{ }^{\circ} \mathrm{C}$ to $70{ }^{\circ} \mathrm{C}$ was set and maintained by built-in Peltier element with temperature sensor inserted in the cuvette department. Measurements at $80^{\circ} \mathrm{C}$ were performed by heating the cuvette department containing a cuvette with the sample to $80^{\circ} \mathrm{C}$ and recording several spectra with $5 \mathrm{~min}$ intervals. Then spectra were normalized to the absorption magnitude of the first record and averaged. All spectra were smoothed using moving average with 3-7 points window and normalized to concentration of oligomer. Acetonitrile was purchased from «AppliChem», Germany. 


\section{$\underline{\text { X-RAY STUDIES }}$}

The molecule D-2d comprises two substituted pyrrolidine cycles linked by nearly planar Zamide bond (Fig. S1). According to CSD data, all bond lengths and angles in D-2d exhibit traditional values for this type of compounds [1]. Terminal $(1 S, 2 R, 5 S)$-menthyl group adopts chair conformation. Amino hydrogen atom $\mathrm{H}^{21}$ is not involved in hydrogen bonding. However, this amino group forms $\mathrm{O}-\mathrm{H}$...N hydrogen bond with solvent methanol molecule.

The molecular backbone of L-3a consists of three substituted pyrrolidine rings linked by two amide group (Fig. S2). The chain is terminated by $(1 R, 2 S, 5 R)$-menthyl group. All bond lengths and angles in L-3a demonstrate ordinary values for organic compounds [1]. Both amide groups are planar within $0.007(2) \AA$ and exhibit Z-configuration. All pyrrolidine cycles adopt an envelope conformation. Flap atoms $\mathrm{C}^{3}, \mathrm{C}^{20}$, and $\mathrm{C}^{35}$ are displaced from the base planes of other four atoms in pyrrolidine cycles by $0.577(3), 0.571(3)$, and $0.567(3) \AA$, respectively. Terminal menthol ring adopts chair conformation with all substituents occupying equatorial positions. Of interest, amino group $\mathrm{N}^{3}-\mathrm{H}^{3}$ does not participate in any hydrogen bonding. The adjacent molecules in the structure L-3a are linked by weak van der Waals interactions only. In crystal, there are wide channels passing along $a$-axis (Fig. S3). These channels are filled by disordered solvent DMSO molecules.

The crystals of D-4aA were obtained from methanol solution on cooling. The molecular structure of tetramer D-4aA (Fig. S4) is similar to that of trimetric L-3a. Amide group with central N(1) atom exhibits E-configuration, while two others adopt Z-arrangement. As for L-3a, amino group $\mathrm{N}^{4}-\mathrm{H}^{42}$ does not form any hydrogen bond. In crystal, there are wide channels passing along a-axis (Fig. S5). These channels are filled by solvent methanol and partially occupied water molecules. Solvent molecules form donor hydrogen bonds $\mathrm{OH}$... $\mathrm{O}=\mathrm{C}$ with all amide groups of the main molecule $\left(\mathrm{O}^{2}, \mathrm{O}^{5}\right.$ and $\mathrm{O}^{8}$ atoms).

The crystals of $\mathbf{D}-\mathbf{4 a B}$ were obtained from ethyl acetate solution by slow evaporation. The molecular structures D-4aA and D-4aB (Fig. S6) are very close to each other. The orthogonal fitting of both chemically identical molecules are shown on Fig. S7. The main difference is observed for conformation of terminal menthol substituent. In crystal D-4aB, we also observe wide channels passing along $a$-axis (Fig. S8). These channels are filled by solvent ethyl acetate molecules. Comparing cell dimension from Table S1, one can assume that the structures D-4aA and D-4aB may be treated as quasi-isostructural ( $a=11.6250$ vs. $11.6415 \AA, b=18.3893$ vs. 
$18.4064 \AA$, doubling $c=14.4844 \times 2=28.9688$ vs. $30.1665 \AA, \beta=92.410$ vs. $90^{\circ}$ ). Surprisingly, that is not so. In both structures there is just one crystallographically independent molecule ( $Z^{\prime}$ =1). Thus, in crystal D-4aA any interatomic vector for all molecules form the same angle with $b$ axis since $12_{1} 1$ is the only nonidentical symmetry operation. Contrarily, in $\mathbf{D}-\mathbf{4 a B}$ there are three distinct symmetry operations 21 along all three axis. For instance, $\mathrm{N}^{2} \rightarrow \mathrm{N}^{4}$ vectors in four translationally independent molecules form the angles 58.9, 121.1, 58.9 and 121.1 with $b$-axis. It should be noted that such pseudo-isomorphism is quite rare $[2,3]$.

Experimental data for D-2d and L-3a was measured on a Bruker SMART APEX II diffractometer using graphite monochromatized MoK $\alpha$ radiation $(\lambda=0.71073 \AA$ ) at $180 \mathrm{~K}$. Intensities for $\mathbf{D}-4 \mathrm{aA}$ and D-4aB were collected on a Bruker D8 Venture machine using graphite monochromatized CuK $\alpha$ radiation $(\lambda=1.54178 \AA$ ) at $100 \mathrm{~K}$. The structures were solved by direct methods and refined by full matrix least-squares on $F^{2}$ [4] with anisotropic thermal parameters for all nonhydrogen atoms (except partially occupied water positions in D-4aA). In D-4aB, solvent ethyl acetate molecules were not located and their contribution was suppressed by the SQUEEZE procedure [5]. For these structures, all hydrogen atoms were placed in calculated positions and refined using a riding model. In the structure L-3a, sulfur atom of solvent DMSO molecule is disordered over two positions with occupancy ratio 0.82 / 0.18.

The crystallographic data for D-2d, L-3a, D-4aA and D-4aB (Table S1) have been deposited with the Cambridge Crystallographic Data Centre as supplementary publications under the CCDC numbers 1032603, 981128, 981129 and 981130, respectively. They can be obtained free of charge from the Cambridge Crystallographic Data Centre via www.ccdc.cam.ac.uk/data request/cif. 
Table S1. Crystallographic data and refinement details for compounds D-2d, L-3a, D-4a.

\begin{tabular}{|c|c|c|c|c|}
\hline & $D-2 d$ & L-3a & $\begin{array}{c}\text { D-4aA } \\
\text { (methanol/water } \\
\text { solvate) }\end{array}$ & $\begin{array}{c}\text { D-4aB (ethyl } \\
\text { acetate solvate) }\end{array}$ \\
\hline \multirow[t]{2}{*}{ Formula } & $\mathrm{C}_{37} \mathrm{H}_{47} \mathrm{ClN}_{2} \mathrm{O}_{8}$ & $\mathrm{C}_{49} \mathrm{H}_{59} \mathrm{~N}_{3} \mathrm{O}_{10}$. & $\mathrm{C}_{62} \mathrm{H}_{72} \mathrm{~N}_{4} \mathrm{O}_{13}$ & $\mathrm{C}_{62} \mathrm{H}_{72} \mathrm{~N}_{4} \mathrm{O}_{13}$ \\
\hline & $2 \mathrm{CH}_{3} \mathrm{OH}$ & $\mathrm{C}_{2} \mathrm{H}_{6} \mathrm{OS}$ & $\mathrm{CH}_{3} \mathrm{OH} \cdot 1.6 \mathrm{H}_{2} \mathrm{O}$ & \\
\hline Formula weight & 747.30 & 928.12 & 1142.10 & 1081.24 \\
\hline $\begin{array}{l}\text { Crystal size } \\
/ \mathrm{mm}^{3}\end{array}$ & $0.30 \times 0.15 \times 0.05$ & $0.30 \times 0.20 \times 0.20$ & $0.06 \times 0.03 \times 0.005$ & $0.18 \times 0.08 \times 0.01$ \\
\hline Crystal system & orthorhombic & orthorhombic & monoclinic & orthorhombic \\
\hline Space group & $P 2{ }_{1}{ }_{1} 2_{1}$ & $P 2_{1} 2_{1} 2_{1}$ & $P 2_{1}$ & $P 2_{1} 2_{1} 2_{1}$ \\
\hline Z & 4 & 4 & 2 & 4 \\
\hline$a / \AA$ & $10.6843(11)$ & $10.0307(7)$ & $11.6250(5)$ & $11.6415(2)$ \\
\hline$b / \AA ̊$ & $11.0107(11)$ & $20.7683(15)$ & $18.3893(8)$ & $18.4064(3)$ \\
\hline$c / \AA ̊$ & $33.664(4)$ & $24.3956(18)$ & $14.4844(7)$ & $30.1665(5)$ \\
\hline$\beta /^{\circ}$ & 90 & 90 & $92.410(3)$ & 90 \\
\hline Volume $/ \AA^{3}$ & $3960.3(7)$ & $5082.1(6)$ & $3093.7(2)$ & $6464.02(19)$ \\
\hline Total reflections & 33990 & 48292 & 17693 & 39336 \\
\hline $\begin{array}{l}\text { Unique data } \\
\left.\text { ( } R_{\text {int }}\right)\end{array}$ & $9550(0.0414)$ & $11067(0.0376)$ & 9191 (0.1091) & $10427(0.0542)$ \\
\hline$\vartheta /{ }^{\circ}$ & 2.21 to 28.00 & 1.94 to 26.99 & 3.05 to 64.98 & 2.81 to 64.99 \\
\hline $\begin{array}{l}\text { Data/restraints/ } \\
\text { parameters }\end{array}$ & 9550 / 0 / 479 & 11067 / 7 / 613 & 9191 / 1 / 750 & 10427 / 6 / 723 \\
\hline GoF on $F^{2}$ & 1.026 & 1.068 & 1.018 & 1.037 \\
\hline$R_{1}[I>2 \sigma(I)]$ & 0.0573 & 0.0449 & 0.0782 & 0.0401 \\
\hline$w R_{2}$ (all data) & 0.1558 & 0.0999 & 0.1955 & 0.0958 \\
\hline $\begin{array}{l}\text { Largest diff. } \\
\text { peak and hole, } \\
e \cdot \AA^{-3}\end{array}$ & 0.985 / -0.295 & $0.392 /-0.503$ & $0.575 /-0.274$ & $0.289 /-0.133$ \\
\hline
\end{tabular}

References

(1) Allen, F.H. Acta Cryst. 2002, B58, Part 3, No 1, 380-388.

(2) Centore, R.; Fusco, S.; Jazbinsek, M.; Capobianco, A.; Peluso, A. CrystEngComm 2013, 15, 3318-3325.

(3) Vogt, F. G.; Copley, R. C. B.; Mueller, R. L.; Spoors, G. P.; Cacchio, T. N.; Carlton, R. A.; Katrincic, L. M.; Kennady, J. M.; Parsons, S.; Chetina, O. V. Cryst. Growth Des. 2010, 10, 2713-2733.

(4) Sheldrick, G. M. Acta Cryst. 2008, A64, No 1, 112-122.

(5) Spek, A. L. J. Appl. Cryst. 2003, 36, No 1, 7-13. 


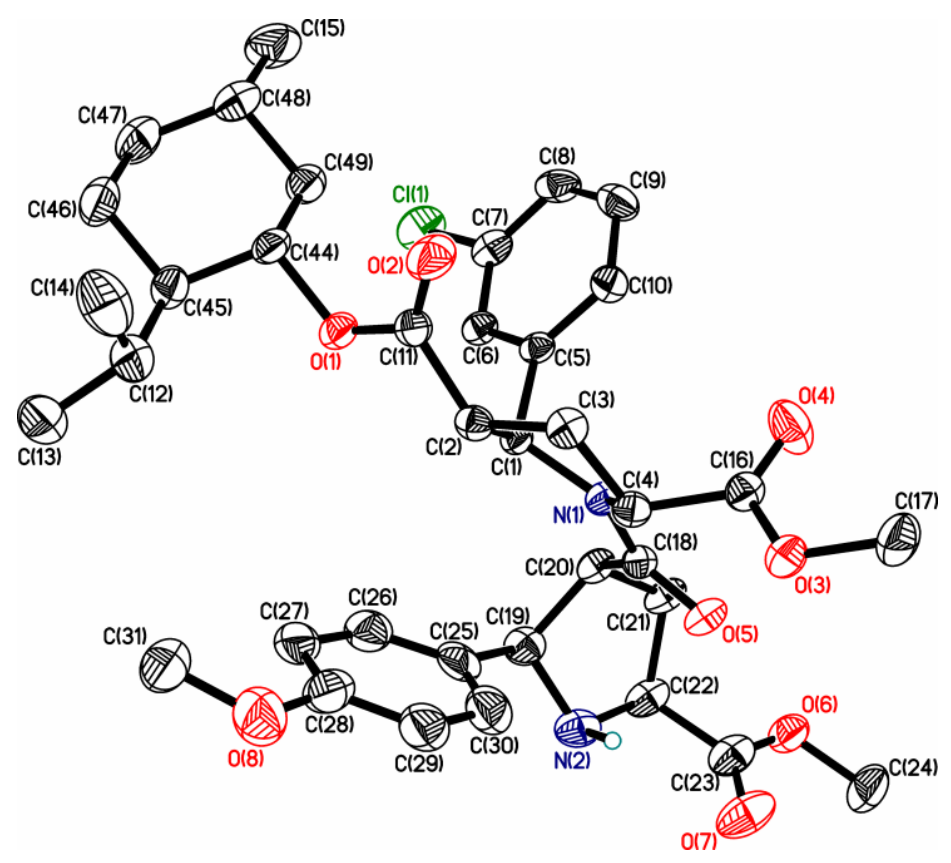

Figure S1. Molecular structure of D-2d. Displacement ellipsoids are shown at 50\% probability level. Hydrogen atoms are omitted for clarity.

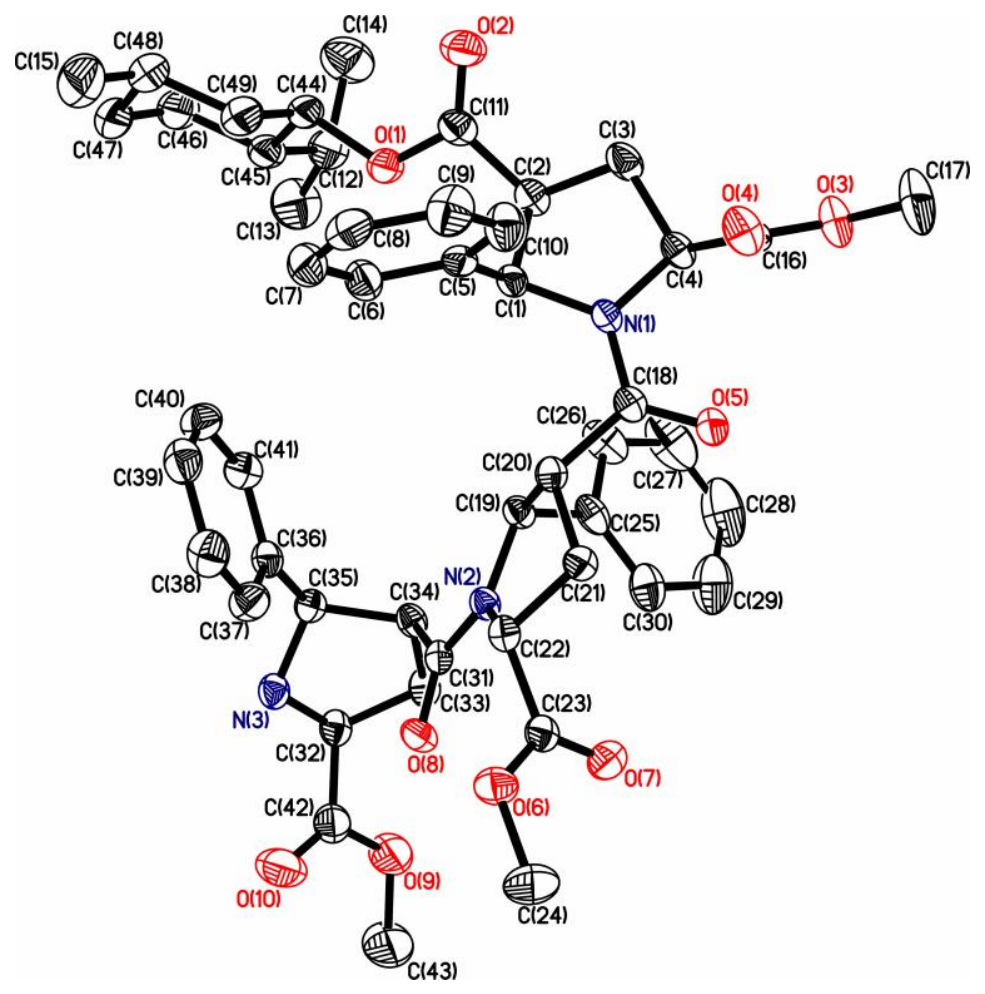

Figure S2. Molecular structure of L-3a. Displacement ellipsoids are shown at 50\% probability level. Hydrogen atoms are omitted for clarity. 


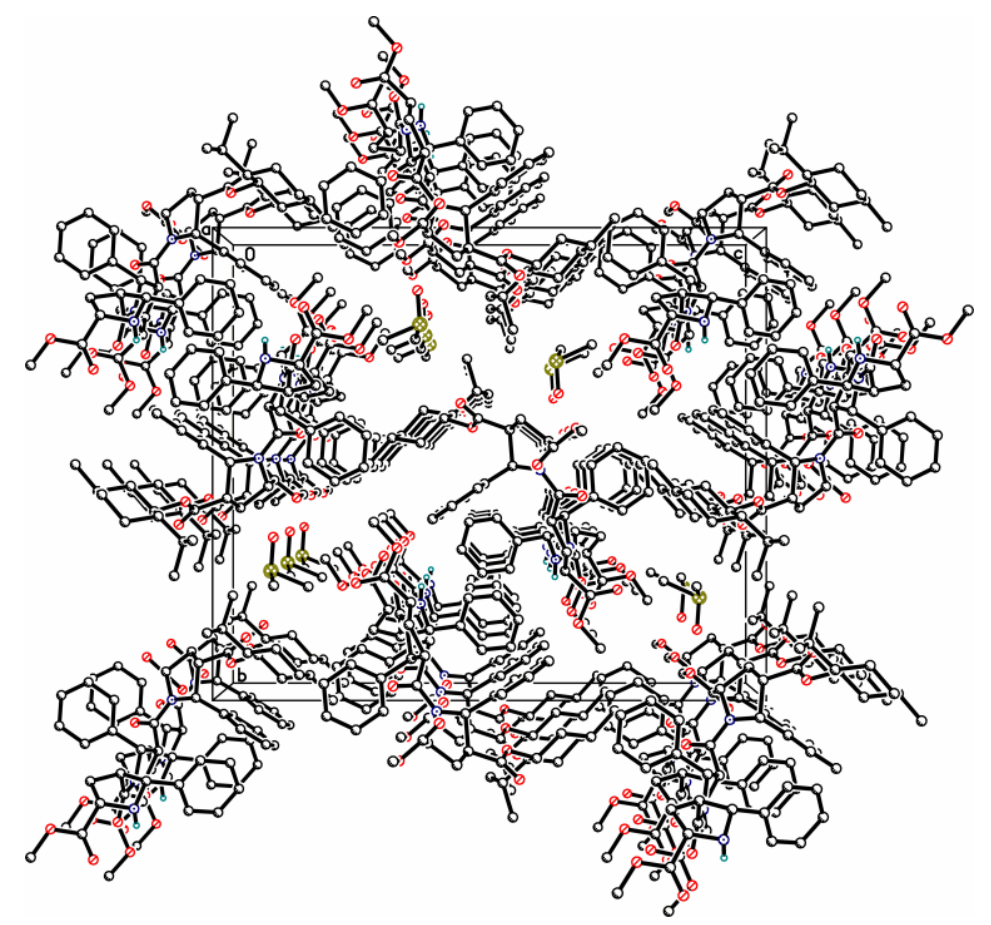

Figure S3. Crystal packing in the structure L-3a.

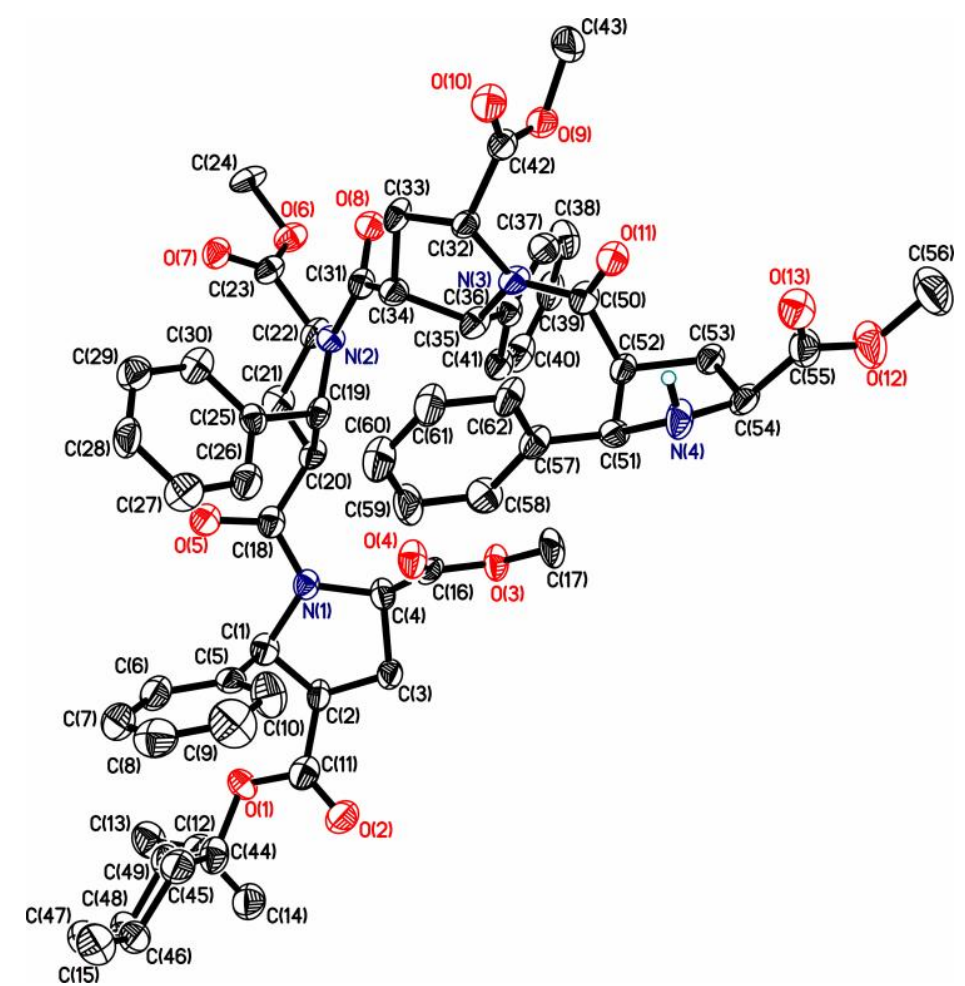

Figure S4. Molecular structure of D-4aA. Displacement ellipsoids are shown at $50 \%$ probability level. Hydrogen atoms (except amino $\mathrm{H}^{42}$ ) are omitted for clarity. 


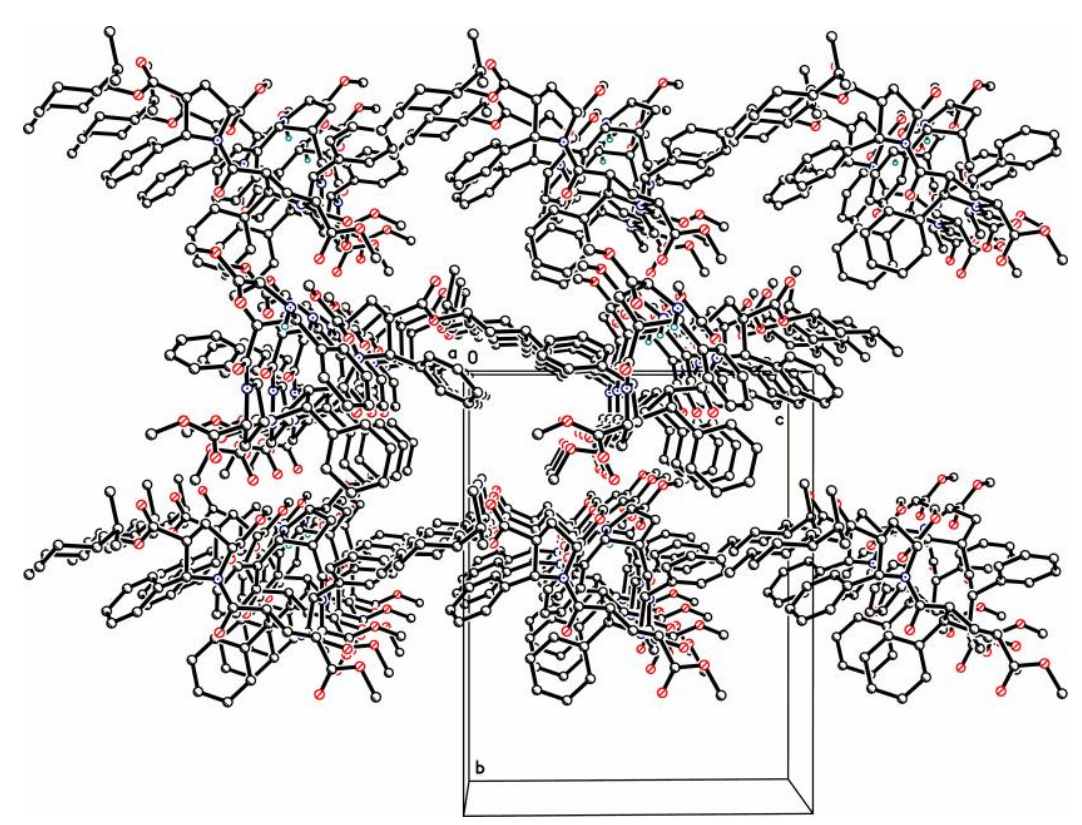

Figure S5. Channels passing along $a$-axis in the structure D-4aA. Solvent methanol and partially occupied water molecules are not shown for clarity.

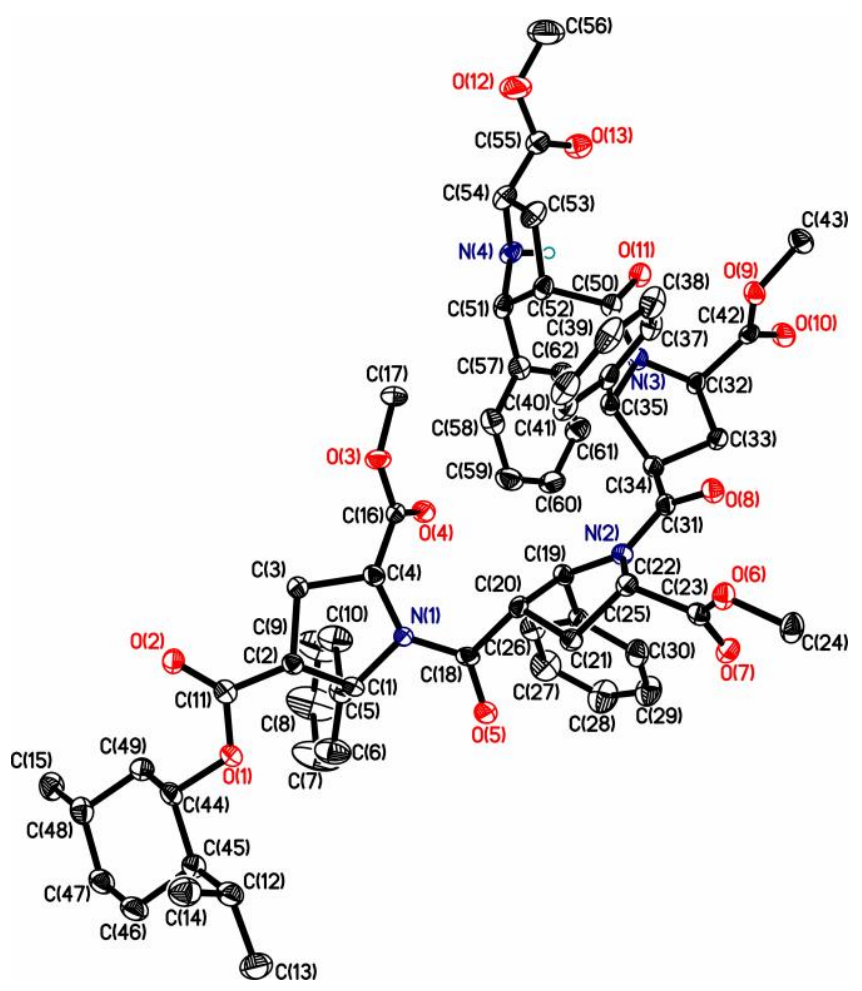

Figure S6. Molecular structure of D-4aB. Displacement ellipsoids are shown at $50 \%$ probability level. Hydrogen atoms (except amino $\mathrm{H}^{42}$ ) are omitted for clarity. 


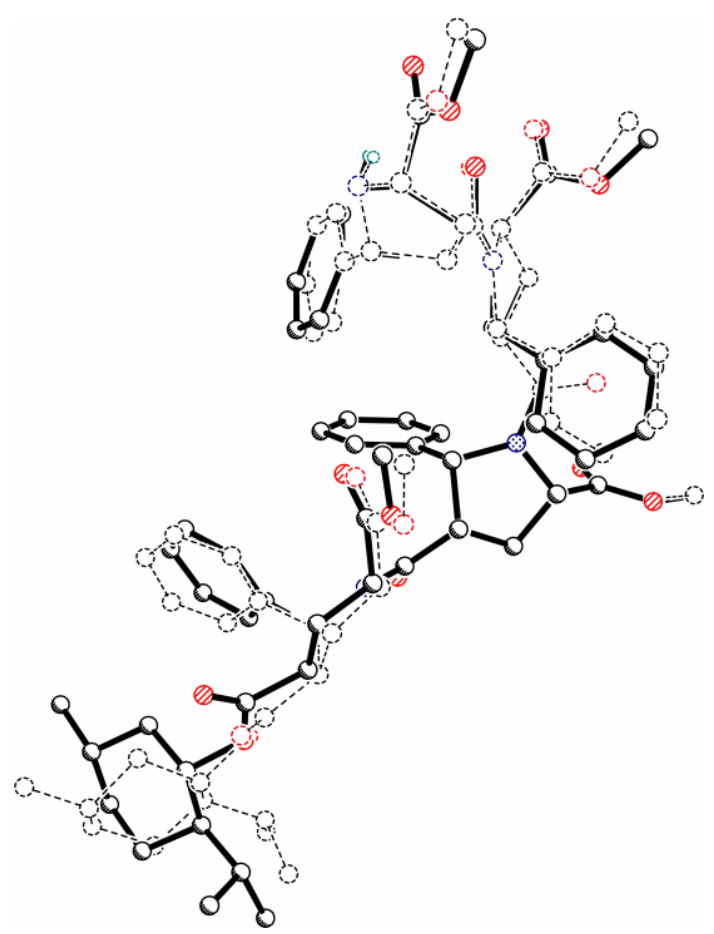

Figure S7. Orthogonal fitting of the molecules D-4aA and D-4aB.

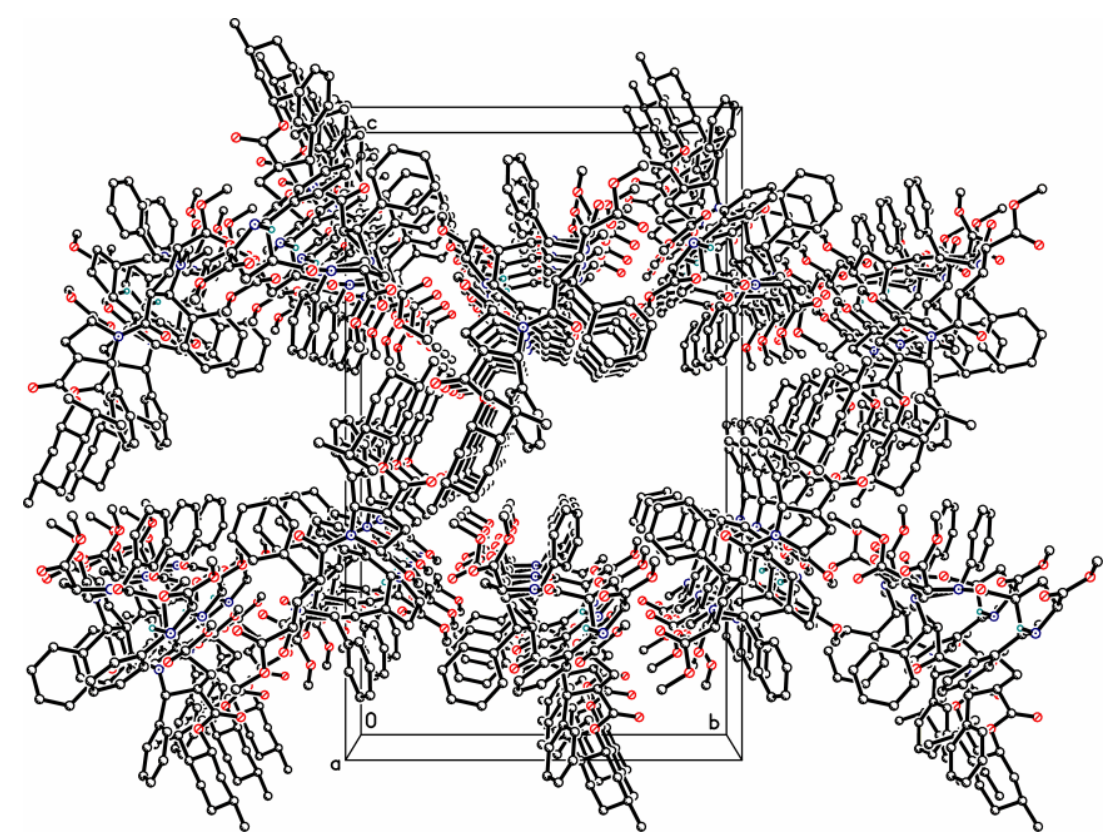

Figure S8. Channels passing along $a$-axis in the structure D-4aB. Solvent ethyl acetate molecules are not shown. 


\section{NMR STUDIES}

Table S2. Pyrrolidine rings signals assignment in ${ }^{1} \mathrm{H}$ NMR spectra of the dominant conformers of oligomers D-4a, D-5a, D-6a.

\begin{tabular}{|c|c|c|c|c|c|}
\hline $\begin{array}{l}\text { Major conformer } \\
\text { of tetramer } \mathbf{D}-\mathbf{4} a^{*}\end{array}$ & $\mathrm{H}^{\alpha}(\mathrm{HCPh})$ & $\begin{array}{l}\mathrm{H}^{\delta} \\
\left(\mathrm{HCCO}_{2} \mathrm{Me}\right)\end{array}$ & $\begin{array}{l}\mathrm{H}^{\beta}(\mathrm{HCC}(=\mathrm{O}) \mathrm{N} \\
\text { or } \\
\left.\mathrm{HCO}_{2} \text { Menthyl }\right)\end{array}$ & $\mathrm{H}^{\gamma^{1}}(\mathrm{HCH})$ & $\mathrm{H}^{\gamma^{2}}(\mathrm{HCH})$ \\
\hline $\begin{array}{l}\text { Pyrrolidine ring } \\
\text { (4), C-end }\end{array}$ & 5.58 & 3.52 & 3.82 & 2.36 & 2.21 \\
\hline Pyrrolidine ring (3) & 5.49 & 3.36 & 3.20 & 2.03 & 1.79 \\
\hline Pyrrolidine ring (2) & 4.94 & 3.38 & 2.63 & 2.08 & 1.61 \\
\hline $\begin{array}{l}\text { Pyrrolidine ring } \\
\text { (1), N-end }\end{array}$ & 4.42 & 3.75 & 3.03 & 2.02 & 1.85 \\
\hline $\begin{array}{l}\text { Major conformer } \\
\text { of pentamer } D \text { - } \\
5 a^{*}\end{array}$ & $\mathrm{H}^{\alpha}(\mathrm{HCPh})$ & $\begin{array}{l}\mathrm{H}^{\delta} \\
\left(\mathrm{HCCO}_{2} \mathrm{Me}\right)\end{array}$ & $\begin{array}{l}\mathrm{H}^{\beta}(\mathrm{HCC}(=\mathrm{O}) \mathrm{N} \\
\text { or } \\
\left.\mathrm{HCO}_{2} \text { Menthyl }\right)\end{array}$ & $\mathrm{H}^{\gamma^{1}}(\mathrm{HCH})$ & $\mathrm{H}^{\mathrm{V}^{2}}(\mathrm{HCH})$ \\
\hline $\begin{array}{l}\text { Pyrrolidine ring } \\
\text { (5), C-end }\end{array}$ & 4.85 & 3.63 & 2.53 & 2.07 & 2.00 \\
\hline Pyrrolidine ring (4) & 5.45 & 5.51 & 3.15 & 2.54 & 2.31 \\
\hline Pyrrolidine ring (3) & 5.67 & 3.66 & 2.83 & 2.17 & 1.98 \\
\hline Pyrrolidine ring (2) & 4.85 & 3.64 & 2.5 & 2.08 & 1.99 \\
\hline $\begin{array}{l}\text { Pyrrolidine ring } \\
\text { (1), N-end }\end{array}$ & 4.75 & 3.22 & 2.98 & 2.10 & 1.72 \\
\hline $\begin{array}{l}\text { Major conformer } \\
\text { of hexamer D-6a* }\end{array}$ & $\mathrm{H}^{\alpha}(\mathrm{HCPh})$ & $\begin{array}{l}\mathrm{H}^{\delta} \\
(\mathrm{HCCO} \\
2 \mathrm{Me})\end{array}$ & $\begin{array}{l}\mathrm{H}^{\beta}(\mathrm{HCC}(=\mathrm{O}) \mathrm{N} \\
\text { or } \\
\left.\mathrm{HCO}_{2} \text { Menthyl }\right)\end{array}$ & $\mathrm{H}^{\gamma 1}(\mathrm{HCH})$ & $\mathrm{H}^{\mathrm{Y} 2}(\mathrm{HCH})$ \\
\hline $\begin{array}{l}\text { Pyrrolidine ring } \\
\text { (6), C-end }\end{array}$ & 5.87 & 3.83 & 3.68 & 2.32 & 2.20 \\
\hline Pyrrolidine ring (5) & 5.65 & 3.21 & 2.88 & 1.98 & 1.53 \\
\hline Pyrrolidine ring (4) & 5.71 & 5.38 & 3.11 & 2.58 & 2.25 \\
\hline Pyrrolidine ring (3) & 5.69 & 3.60 & 3.16 & 2.21 & 2.08 \\
\hline Pyrrolidine ring (2) & 5.22 & 3.67 & 2.70 & 2.06 & 1.68 \\
\hline $\begin{array}{l}\text { Pyrrolidine ring } \\
\text { (1), N-end }\end{array}$ & 4.74 & 3.64 & 2.96 & 2.08 & 1.72 \\
\hline $\begin{array}{l}\text { Major conformer } \\
\text { of hexamer } D- \\
6 a^{* *}\end{array}$ & $\begin{array}{l}H^{\alpha}(H C P h) \\
\text { (d) }\end{array}$ & $\begin{array}{l}\mathrm{H}^{\delta} \\
\left(\mathrm{HCCO}_{2} \mathrm{Me}\right) \\
\text { (dd) }\end{array}$ & $\begin{array}{l}\mathrm{H}^{\beta}(\mathrm{HCC}(=\mathrm{O}) \mathrm{N} \\
\text { or } \\
\mathrm{HCO}_{2} \text { Menthyl) } \\
\text { (ddd) }\end{array}$ & $\begin{array}{l}\mathrm{H}^{\gamma^{1}}(\mathrm{HCH}) \\
\text { (ddd) }\end{array}$ & $\begin{array}{l}\mathrm{H}^{\mathrm{Y}^{2}}(\mathrm{HCH}) \\
\text { (ddd) }\end{array}$ \\
\hline $\begin{array}{l}\text { Pyrrolidine ring } \\
\text { (6), C-end }\end{array}$ & 5.23 & 3.80 & 3.22 & 2.35 & 2.25 \\
\hline \multicolumn{6}{|c|}{ J-couplings: $\mathrm{H}^{\alpha}-\mathrm{H}^{\beta} 8.5 \mathrm{~Hz} ; \mathrm{H}^{\beta}-\mathrm{H}^{\gamma^{1}} 12.9 \mathrm{~Hz} ; \mathrm{H}^{\beta}-\mathrm{H}^{{ }^{2}} 6.5 \mathrm{~Hz}$} \\
\hline Pyrrolidine ring (5) & 5.03 & 3.72 & 2.91 & 2.27 & 1.65 \\
\hline \multicolumn{6}{|c|}{ J-couplings: $\mathrm{H}^{\alpha}-\mathrm{H}^{\beta} 8.4$} \\
\hline Pyrrolidine ring (4) & 5.49 & 4.82 & 3.14 & 2.90 & 2.39 \\
\hline
\end{tabular}




\begin{tabular}{|c|c|c|c|c|c|}
\hline Pyrrolidine ring (3) & 5.70 & 3.75 & 3.28 & 2.32 & 2.18 \\
\hline \multicolumn{6}{|c|}{ J-couplings: $\mathrm{H}^{\alpha}-\mathrm{H}^{\beta} 7.3 \mathrm{~Hz} ; \mathrm{H}^{\beta}-\mathrm{H}^{\gamma 1} 12.7 ; \mathrm{H}^{\beta}-\mathrm{H}^{\gamma^{2}} 5.4 \mathrm{~Hz}$} \\
\hline Pyrrolidine ring (2) & 5.16 & 3.37 & 2.76 & 2.32 & 1.76 \\
\hline \multicolumn{6}{|c|}{ J-couplings: $\mathrm{H}^{\alpha}-\mathrm{H}^{\beta} 8.8 \mathrm{~Hz} ; \mathrm{H}^{\delta}-\mathrm{H}^{\gamma^{1}} 11.2 \mathrm{~Hz} ; \mathrm{H}^{\delta}-\mathrm{H}^{\gamma^{2}} 7.0 \mathrm{~Hz} ; \mathrm{H}^{\beta}-\mathrm{H}^{\gamma^{1}} 12.1 \mathrm{~Hz} ; \mathrm{H}^{\beta}-\mathrm{H}^{\gamma^{2}} 6.6 \mathrm{~Hz}$} \\
\hline $\begin{array}{l}\text { Pyrrolidine ring } \\
(1), N \text {-end }\end{array}$ & 4.70 & 3.69 & 3.06 & 2.25 & 1.93 \\
\hline
\end{tabular}

* - DMSO as a solvent

$* *-\mathrm{CD}_{2} \mathrm{Cl}_{2}$ as a solvent

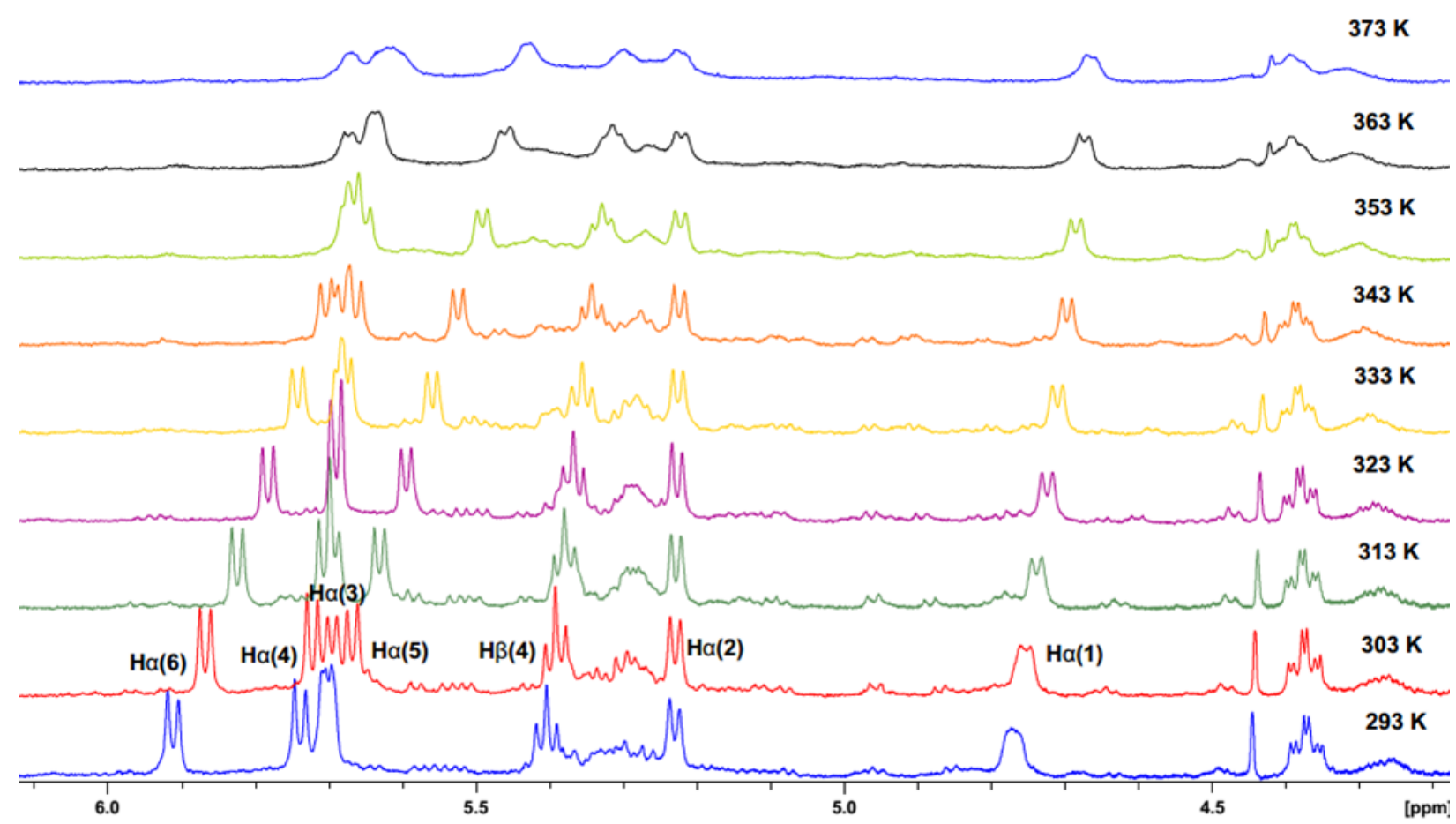

Figure S9. Region of ${ }^{1} \mathrm{H}$ NMR spectrum of hexamer $\mathbf{D}-6 \mathrm{a}$ at different temperatures with signals of $\mathrm{H}^{\alpha}$-protons. 


\section{$\underline{\text { CD STUDIES }}$}

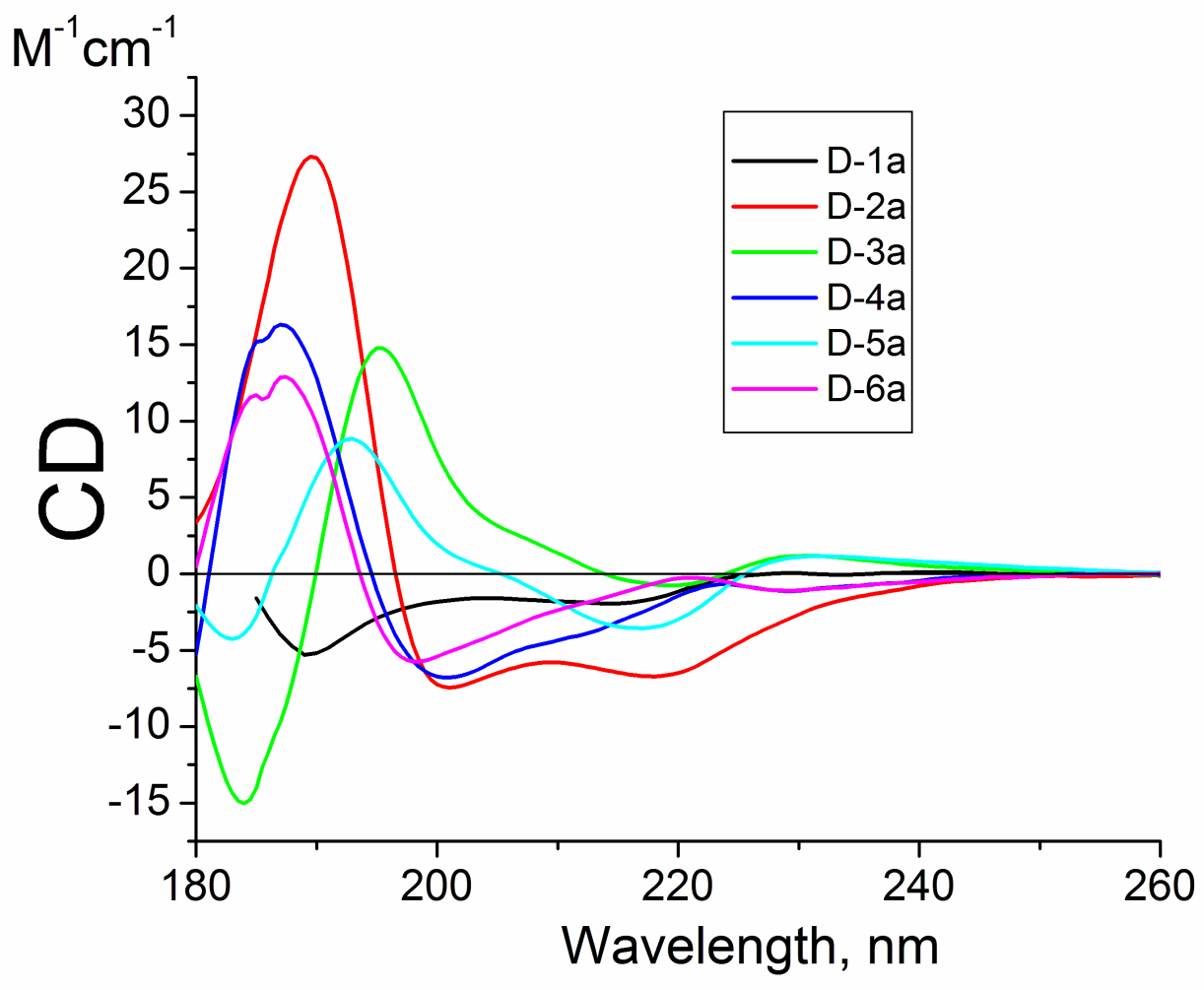

Figure S10. CD spectra of oligomers D-na in acetonitrile solution normalized with respect to concentration and number of monomeric units.

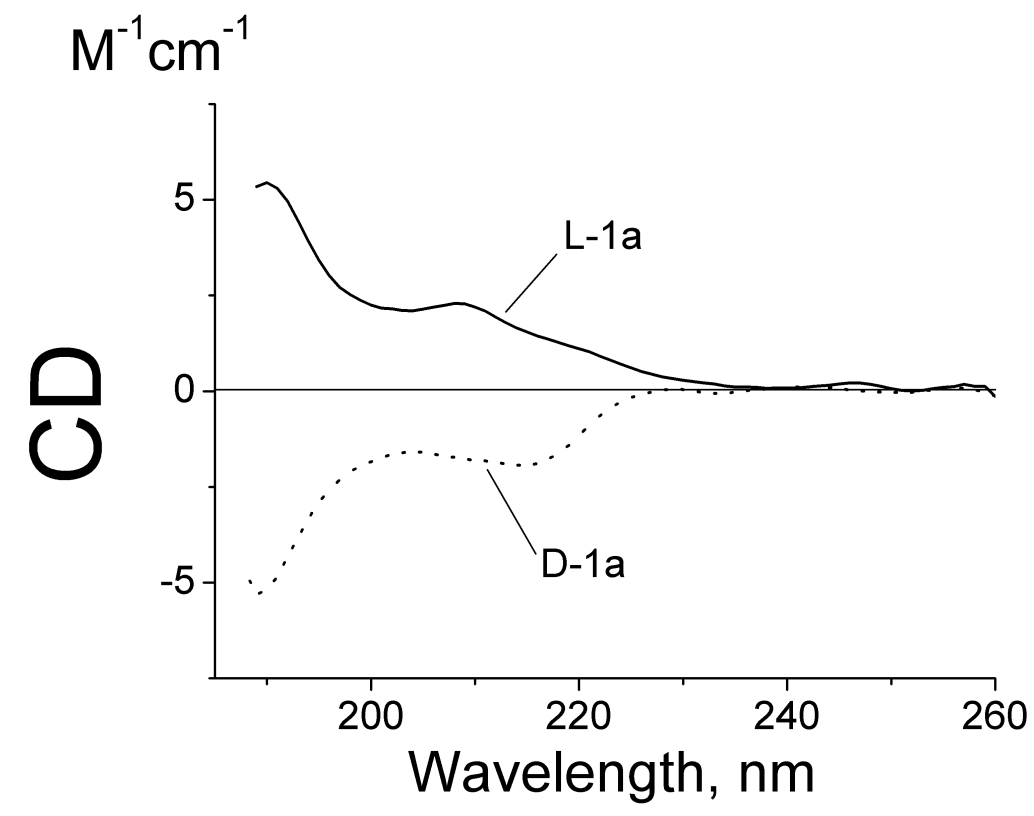

Figure S11. CD spectra of compounds D-1a and L-1a in acetonitrile normalized to samples concentration. 

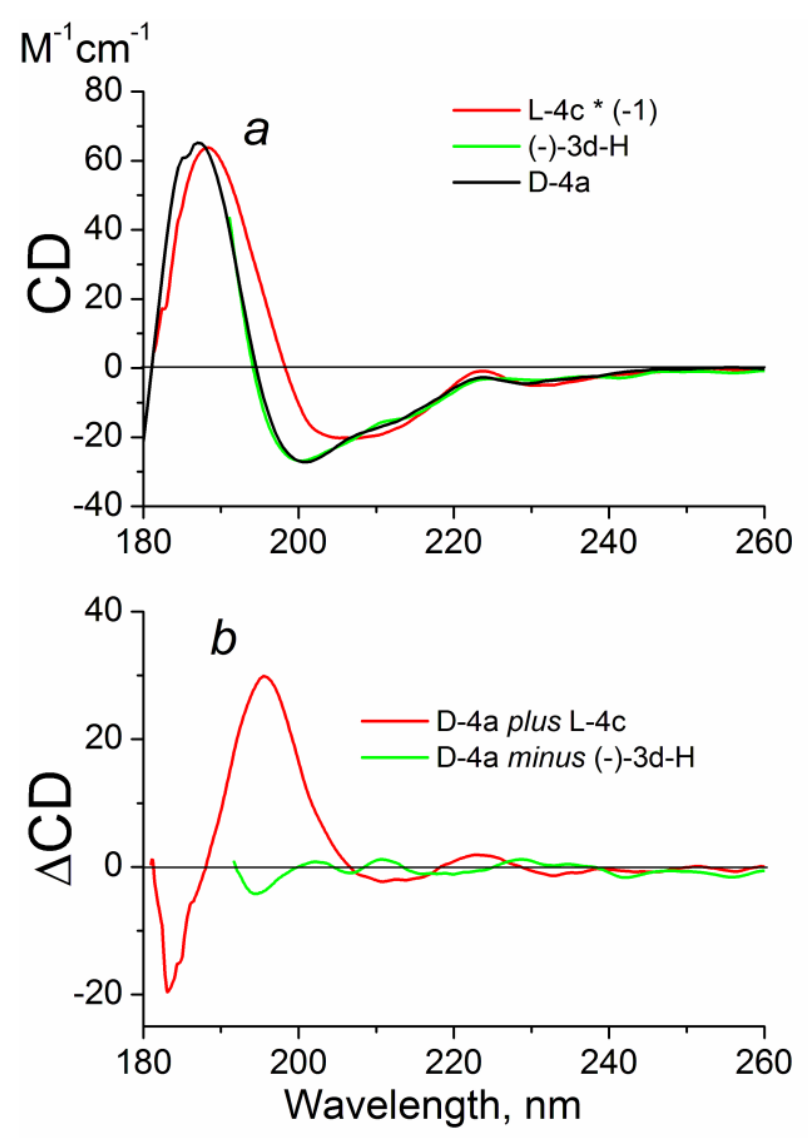

Figure S12. (a) CD spectra of tetramers D-4a, L-4c (multiplied by -1) and (-)-3d-H [Kudryavtsev, K. V.; Ivantcova, P. M.; Churakov, A. V.; Wiedmann, S.; Luy, B.; Muhle-Goll, C.; Zefirov, N. S.; Bräse, S. Angew. Chem. Int. Ed. 2013, 52, 12736-12740] in acetonitrile normalized to samples concentration; $(b)$ difference curves obtained by subtracting spectrum lines $\mathbf{L}-\mathbf{4 c}$ and $(-\mathbf{-}-\mathbf{- 3 d} \mathbf{d}-\mathbf{H}$ from D-4a spectrum line.

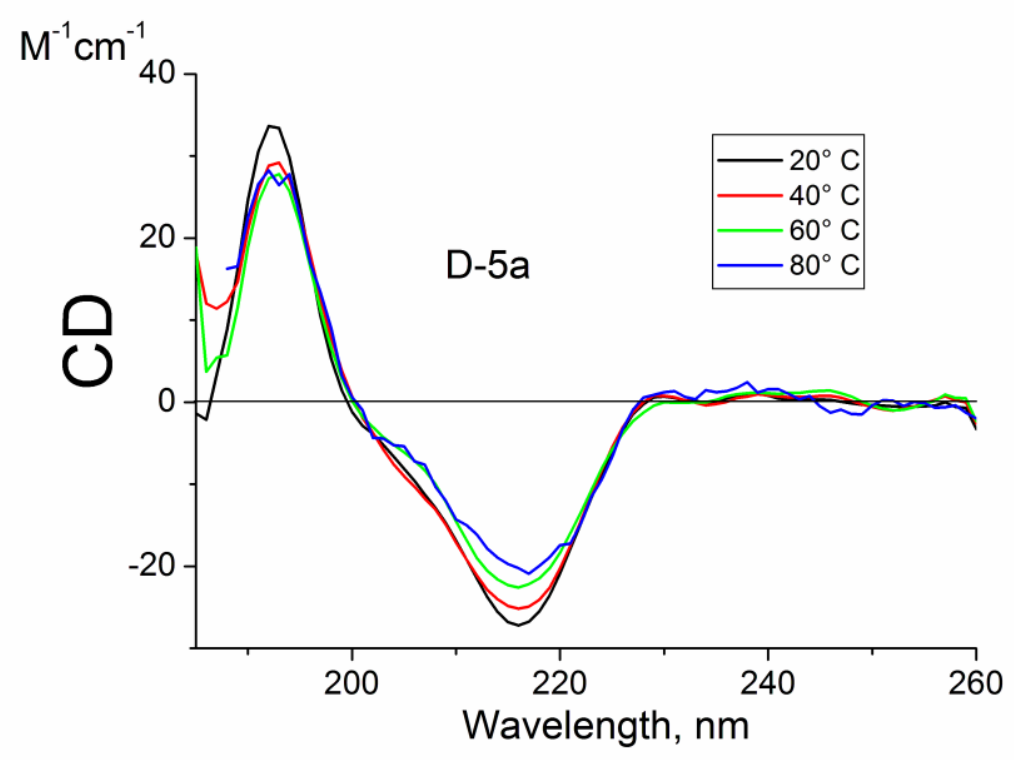

Figure S13. CD spectra of pentamer D-5a at different temperatures in acetonitrile solution. 

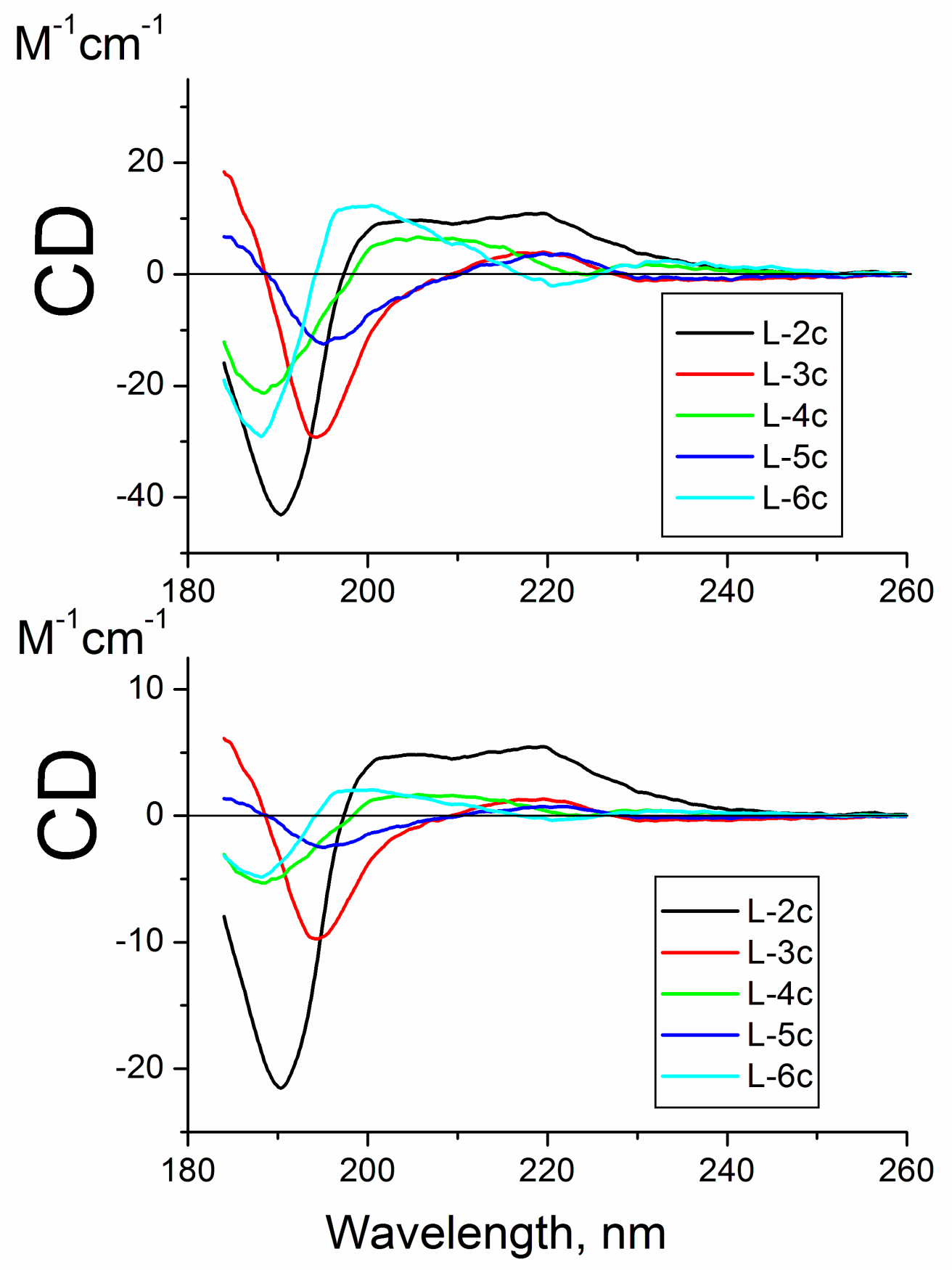

Figure S14. CD spectra of oligomers L-cn in acetonitrile normalized only with respect to concentration (top) and with respect to both concentration and number of monomeric units (bottom). 


\section{BIOLOGICAL STUDIES}

\section{Cell line and cell culture}

PC-3 cells were from American Type Culture Collection (Rockville, MD). Cells were cultured in RPMI-1640 medium supplemented with $10 \%$ heat-inactive fetal bovine serum $(\mathrm{v} / \mathrm{v})$, penicillin $(100$ units $/ \mathrm{ml})$ and streptomycin $(100 \mu \mathrm{g} / \mathrm{ml})$. Cultures were maintained in an incubator with $5 \% \mathrm{CO}_{2}$ at $37^{\circ} \mathrm{C}$.

\section{Sulforhodamine B assay}

Cells were seeded in 96 -well plates in medium with $10 \%$ fetal bovine serum. After 24 hours, cells were fixed with $10 \%$ trichloroacetic acid to represent cell population at the time of compound addition ( $\left.\mathrm{T}_{0}\right)$. After additional incubation of $0.1 \%$ dimethylsulfoxide (DMSO) or the indicated compound for 48 hours, the cells were fixed with $10 \%$ trichloroacetic acid and sulforhodamine $B$ at $0.4 \%(\mathrm{w} / \mathrm{v})$ in $1 \%$ acetic acid was added to stain cells. Unbound sulforhodamine B was washed out by $1 \%$ acetic acid. Sulforhodamine B bound cells were solubilized with $10 \mathrm{mM}$ Trizma base. The absorbance was read at a wavelength of $515 \mathrm{~nm}$. Using the following absorbance measurements, such as time zero $\left(T_{0}\right)$, control growth $(C)$, and cell growth in the presence of the indicated compound (Tx), the percentage growth was calculated at each of the compound concentrations levels. Percentage growth inhibition was calculated as: [1- $\left.\left(\mathrm{Tx}-\mathrm{T}_{0}\right) /\left(\mathrm{C}-\mathrm{T}_{0}\right)\right] \times 100 \%$. Growth inhibition of $50 \%\left(\mathrm{Gl}_{50}\right)$ is determined at the compound concentration which results in $50 \%$ reduction of total protein increase in control cells during the compound incubation.

\section{Flow cytometric assay of propidium iodide staining}

After the exposure to the indicated agent, cells were harvested by trypsinization, fixed with 70 $\%(v / v)$ alcohol at $4^{\circ} \mathrm{C}$ for 30 minutes and washed with phosphate-buffered saline (PBS). The cells were centrifuged and resuspended with $0.5 \mathrm{ml}$ propidium iodide solution containing Triton $\mathrm{X}$-100 $(0.1 \%, v / \mathrm{v})$, RNase $(100 \mu \mathrm{g} / \mathrm{ml})$ and propidium iodide $(80 \mu \mathrm{g} / \mathrm{ml})$. DNA content was analyzed with the FACScan and CellQuest software (Becton Dickinson, Mountain View, CA). 
Table S3. Antiproliferative activity of 3-PCA oligomers

\begin{tabular}{|c|c|c|c|c|c|}
\hline $\begin{array}{c}\text { Compo } \\
\text { und } \\
\text { code }\end{array}$ & $\begin{array}{c}\text { Gl } 50 \text { in PC-3 } \\
\text { cells, } \mu \mathrm{M}^{a}\end{array}$ & $\begin{array}{c}\text { Arrest of cell cycle } \\
\text { progression, } 10 \mu \mathrm{M}, \\
24 \mathrm{~h}\end{array}$ & $\begin{array}{c}\text { Compo } \\
\text { und } \\
\text { code }\end{array}$ & $\begin{array}{c}\text { Gl50 in PC-3 } \\
\text { cells, } \mu \mathrm{M}^{a}\end{array}$ & $\begin{array}{c}\text { Arrest of cell cycle } \\
\text { progression, } 10 \mu \mathrm{M}, \\
24 \mathrm{~h}\end{array}$ \\
\hline L-2a & 6.3 & Go/G1 & L-4a & 9.1 & - \\
\hline L-2b & 7.4 & Go/G1 & L-4b & $>30.0$ & - \\
\hline L-2c & 6.9 & - & L-4c & $>30.0$ & - \\
\hline L-2d & 6.3 & Go/G1 & D-4a & $>30.0$ & - \\
\hline D-2a & 10.2 & Go/G1 & L-5a & 6.6 & - \\
\hline D-2d & 6.4 & Go/G1 & L-5b & $>30.0$ & NT \\
\hline L-3a & 6.4 & subG1; Go/G1 & L-5c & 19.5 & - \\
\hline L-3b & 30.0 & - & D-5a & 25.6 & - \\
\hline L-3c & $>30.0$ & - & L-6a & $>30.0$ & - \\
\hline L-3d & 5.3 & Go/G1 & L-6b & $>30.0$ & NT $^{b}$ \\
\hline D-3a & 4.4 & subG1 & L-6c & 15.2 & - \\
\hline D-3d & 6.0 & G0/G1 & D-6a & $>30.0$ & - \\
\hline
\end{tabular}

${ }^{a}$ Data are expressed as the mean of three determinations. Standard deviation in each case is less than $15 \%$ of mean value. ${ }^{b} \mathrm{NT}-$ not tested. 


\section{GENERAL PROCEDURES}

\section{Synthesis of menthyl acrylates}

Triethylamine (TEA) $(6.70 \mathrm{ml}, 4.86 \mathrm{~g}, 48.0 \mathrm{mmol})$ was added to a stirred solution of L-menthol or D-menthol $\left(5.17 \mathrm{~g}, 33.0 \mathrm{mmol}\right.$ ) in $50 \mathrm{ml} \mathrm{THF}$ at $0{ }^{\circ} \mathrm{C}$. Then the solution of acryloyl chloride $(3.2 \mathrm{ml}, 3.65 \mathrm{~g}, 39 \mathrm{mmol}$ ) in $30 \mathrm{ml} \mathrm{THF}$ was added dropwise to the reaction mixture with the rate not allowing the reaction temperature exceed $5{ }^{\circ} \mathrm{C}$. The mixture was stirred for additional $24 \mathrm{~h}$ at $\mathrm{rt}$. The solution was concentrated under reduced pressure. The residue was diluted by $100 \mathrm{ml} \mathrm{CH}{ }_{2} \mathrm{Cl}_{2}$ and washed subsequently by $\mathrm{H}_{2} \mathrm{O}(50 \mathrm{ml})$ and saturated $\mathrm{NH}_{4} \mathrm{Cl}$ solution $(50 \mathrm{ml})$. Organic phase was dried over $\mathrm{Na}_{2} \mathrm{SO}_{4}$ and concentrated under reduced pressure. The crude products were purified by column chromatography on silica gel using hexane/AcOEt $=10: 1$ as an eluent.

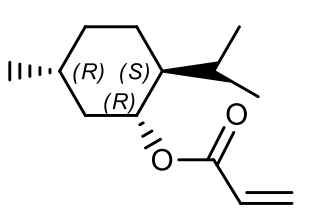

(1R,2S,5R)-2-isopropyl-5-methylcyclohexyl acrylate (L-menthyl acrylate)

Yield $88 \%, 4.800 \mathrm{~g}$, colorless liquid.

${ }^{1} \mathrm{H}$ NMR $\left(400 \mathrm{MHz}, \mathrm{CDCl}_{3}, 293 \mathrm{~K}\right): \delta 0.78(\mathrm{~d}, J=7.0 \mathrm{~Hz}, 3 \mathrm{H}), 0.89-0.93(\mathrm{~m}$, $7 \mathrm{H}), 1.01-1.08(\mathrm{~m}, 2 \mathrm{H}), 1.38-1.59(\mathrm{~m}, 2 \mathrm{H}), 1.67-1.75(\mathrm{~m}, 2 \mathrm{H}), 1.83-1.94(\mathrm{~m}$, 1H), 1.99-2.08 (m, 1H), $4.78(\mathrm{td}, J 10.4,4.4 \mathrm{~Hz}, 1 \mathrm{H}), 5.81$ (dd, J 10.4, $1.5 \mathrm{~Hz}, 1 \mathrm{H}), 6.12$ (dd, J 17.3, $10.4 \mathrm{~Hz}, 1 \mathrm{H}), 6.39$ (dd, J 17.3, $1.5 \mathrm{~Hz}, 1 \mathrm{H})$.

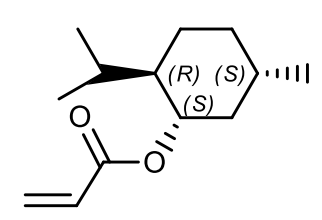

(1S,2R,5S)-2-isopropyl-5-methylcyclohexyl acrylate (D-menthyl acrylate)

Yield 95\%, $7.350 \mathrm{~g}$, colorless liquid.

${ }^{1} \mathrm{H}$ NMR $\left(400 \mathrm{MHz}, \mathrm{CDCl}_{3}, 293 \mathrm{~K}\right): \delta 0.78(\mathrm{~d}, J=7.0 \mathrm{~Hz}, 3 \mathrm{H}), 0.89-0.93(\mathrm{~m}$, $7 \mathrm{H}), 1.01-1.08(\mathrm{~m}, 2 \mathrm{H}), 1.38-1.59(\mathrm{~m}, 2 \mathrm{H}), 1.67-1.75(\mathrm{~m}, 2 \mathrm{H}), 1.83-1.94(\mathrm{~m}$, 1H), 1.99-2.08 (m, 1H), 4.78 (td, J 10.4, $4.4 \mathrm{~Hz}, 1 \mathrm{H}), 5.81$ (dd, J 10.4, 1.5 Hz, 1H), 6.12 (dd, J 17.3, $10.4 \mathrm{~Hz}, 1 \mathrm{H}), 6.39$ (dd, J 17.3, $1.5 \mathrm{~Hz}, 1 \mathrm{H})$.

\section{General procedure for the synthesis of monomers L-1a, L-1b, L-1C, L-1d, D-1a and D-1d.}

A solution of TEA $(1.90 \mathrm{ml}, 1.40 \mathrm{~g}, 14 \mathrm{mmol})$ in $15 \mathrm{ml}$ of toluene was added dropwise to the mixture of corresponding iminoester $\mathrm{ArCH}=\mathrm{NCH}_{2} \mathrm{COOR}(11 \mathrm{mmol}), \mathrm{AgOAc}(2.50 \mathrm{~g}, 14 \mathrm{mmol}), \mathrm{L}-$ menthyl acrylate (or D-menthyl acrylate) $(2.31 \mathrm{~g}, 11 \mathrm{mmol})$ in $75 \mathrm{ml}$ of dry toluene under an inert atmosphere. Reaction flask was protected from the light with aluminium foil. Reaction mixture was stirred at room temperature for $12-48 \mathrm{~h}$. Then the reaction mixture was filtered through Celite. Filtrate was washed with $\mathrm{H}_{2} \mathrm{O}(50 \mathrm{ml})$ and saturated $\mathrm{NH}_{4} \mathrm{Cl}$ solution $(50 \mathrm{ml})$. Organic phase was dried over $\mathrm{Na}_{2} \mathrm{SO}_{4}$ and concentrated under reduced pressure. The crude products were purified by column chromatography on silica gel using hexane/AcOEt 3:1 as an eluent. 
4-((1R,2S,5R)-2-isopropyl-5-methylcyclohexyl) 2-methyl $\quad(2 S, 4 S, 5 R)-5$-phenylpyrrolidine-2,4dicarboxylate L-1a

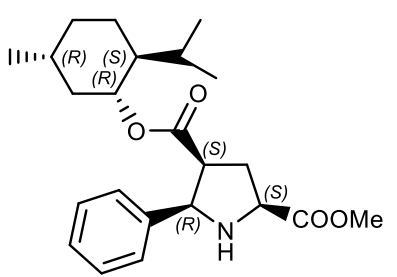

Yield $73 \%, 6.600$ g, colorless crystals, m.p. $103-105{ }^{\circ} \mathrm{C},[\alpha]_{D}{ }^{33}+9.3^{\circ}(c$ 1.13, MeOH). Lit. [1]: m.p. $110^{\circ} \mathrm{C},[\alpha]_{D}-10.6^{\circ}$ (c 1.0, $\left.\mathrm{CH}_{2} \mathrm{Cl}_{2}\right)$.

${ }^{1} \mathrm{H}$ NMR $\left(400 \mathrm{MHz}, \mathrm{DMSO}-\mathrm{d}_{6}, 293 \mathrm{~K}\right): \delta 0.19-0.28(\mathrm{~m}, 1 \mathrm{H}), 0.52(\mathrm{~d}, J 6.9$ $\mathrm{Hz}, 3 \mathrm{H}), 0.60-0.70(\mathrm{~m}, 1 \mathrm{H}), 0.70(\mathrm{~d}, J 6.9 \mathrm{~Hz}, 3 \mathrm{H}), 0.75(\mathrm{~d}, J 6.9 \mathrm{~Hz}, 3 \mathrm{H})$, 0.79-0.86 (m, $1 \mathrm{H}), 0.87-0.95(\mathrm{~m}, 1 \mathrm{H}), 1.04-1.21(\mathrm{~m}, 2 \mathrm{H}), 1.46-1.53(\mathrm{~m}$, $3 \mathrm{H}), 2.17$ (ddd, J 12.8, 8.1, 6.2 Hz, 1H), $2.34(\mathrm{dt}, J 12.8,8.1 \mathrm{~Hz}, 1 \mathrm{H})$, 3.27-3.33 (m, 1H), $3.69(\mathrm{~s}, 3 \mathrm{H}), 3.88(\mathrm{t}, J 8.0 \mathrm{~Hz}, 1 \mathrm{H}), 4.20(\mathrm{td}, J 10.8,4.3 \mathrm{~Hz}, 1 \mathrm{H}), 4.43(\mathrm{~d}, J 8.0$ $\mathrm{Hz}, 1 \mathrm{H}), 7.17-7.21(\mathrm{~m}, 1 \mathrm{H}), 7.24-7.28(\mathrm{~m}, 2 \mathrm{H}), 7.29-7.31(\mathrm{~m}, 2 \mathrm{H})$.

${ }^{13} \mathrm{C}$ NMR (100 MHz, DMSO-d, $\left.293 \mathrm{~K}\right): \delta 16.00,20.48,21.67,22.72,25.45,30.47,32.94,33.55$, $39.36,46.01,48.36,51.74,58.77,64.22,72.86,126.91,127.23$ (2C), 127.74 (2C), 140.18, $171.86,173.47$.

4-((1R,2S,5R)-2-isopropyl-5-methylcyclohexyl)

2-methyl

$(2 S, 4 S, 5 R)-5-(4-$ bromophenyl)pyrrolidine-2,4-dicarboxylate L-1b

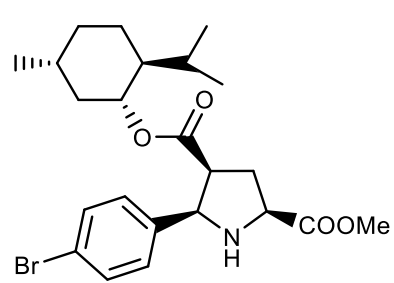

Yield $65 \%, 7.760 \mathrm{~g}$, colorless crystals, m.p. $120-122{ }^{\circ} \mathrm{C},[\alpha]_{D}{ }^{20}+7.1^{\circ}(\mathrm{c}$ 1.13, $\mathrm{MeOH})$

${ }^{1} \mathrm{H}$ NMR $\left(400 \mathrm{MHz}, \mathrm{DMSO}-\mathrm{d}_{6}, 293 \mathrm{~K}\right): \delta 0.17-0.26(\mathrm{~m}, 1 \mathrm{H}), 0.53(\mathrm{~d}, J 6.9$ $\mathrm{Hz}, 3 \mathrm{H}), 0.61-0.70(\mathrm{~m}, 1 \mathrm{H}), 0.75(\mathrm{~d}, J 6.9 \mathrm{~Hz}, 6 \mathrm{H}), 0.81-0.91(\mathrm{~m}, 2 \mathrm{H})$, 1.04-1.11 $(\mathrm{m}, 1 \mathrm{H}), 1.13-1.23(\mathrm{~m}, 1 \mathrm{H}), 1.45-1.53(\mathrm{~m}, 3 \mathrm{H}), 2.17$ (ddd, J $12.7,8.1,6.2 \mathrm{~Hz}, 1 \mathrm{H}), 2.31(\mathrm{dt}, J 12.7,8.1 \mathrm{~Hz}, 1 \mathrm{H}), 3.27-3.33(\mathrm{~m}, 1 \mathrm{H})$, $3.68(\mathrm{~s}, 3 \mathrm{H}), 3.87(\mathrm{t}, J 7.8 \mathrm{~Hz}, 1 \mathrm{H}), 4.21(\mathrm{td}, J 10.8,4.4 \mathrm{~Hz}, 1 \mathrm{H}), 4.44(\mathrm{~d}, J 7.8 \mathrm{~Hz}, 1 \mathrm{H}), 7.25-7.29$ $(\mathrm{m}, 2 \mathrm{H}), 7.43-7.47(\mathrm{~m}, 2 \mathrm{H})$.

${ }^{13} \mathrm{C}$ NMR (100 MHz, DMSO-d 6 , $\left.293 \mathrm{~K}\right): \delta$ 16.01, 20.44, 21.82, 22.73, 25.45, 30.41, 32.44, 33.53, 39.35, 45.97, 48.10, 51.69, 58.67, 63.30, 72.94, 120.13, 129.59 (2C), 130.62 (2C), 139.94, $171.63,173.37$.

Anal. Calcd for $\mathrm{C}_{23} \mathrm{H}_{32} \mathrm{BrNO}_{4}$ : C 59.23, H 6.92, N 3.00. Found: C 59.23, H 7.08, N 2.74.

2-(tert-butyl) 4-((1R,2S,5R)-2-isopropyl-5-methylcyclohexyl) $(2 S, 4 S, 5 R)-5$-phenylpyrrolidine-2,4dicarboxylate $\mathbf{L}-\mathbf{1 C}$

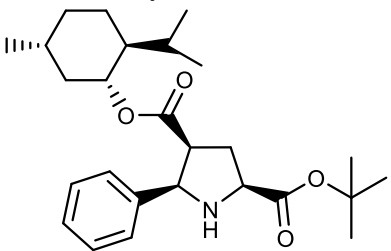

Yield $85 \%, 9.000 \mathrm{~g}$, colorless crystals, m.p. $133-135{ }^{\circ} \mathrm{C},[\alpha]_{D}{ }^{20}+1.4^{\circ}(\mathrm{c}$ 1.13, MeOH). Lit. [2]: m.p. $134-135{ }^{\circ} \mathrm{C},[\alpha]_{D}-29.5^{\circ}(c=2.0 \mathrm{mg} / \mathrm{mL}$, $\left.\mathrm{CHCl}_{3}\right)$.

${ }^{1} \mathrm{H}$ NMR $\left(400 \mathrm{MHz}, \mathrm{CDCl}_{3}, 293 \mathrm{~K}\right): \delta 0.26-0.35(\mathrm{~m}, 1 \mathrm{H}), 0.52(\mathrm{~d}, J 6.9 \mathrm{~Hz}$, $3 \mathrm{H}), 0.62-0.71(\mathrm{~m}, 1 \mathrm{H}), 0.70(\mathrm{~d}, J 6.9 \mathrm{~Hz}, 3 \mathrm{H}), 0.74(\mathrm{~d}, J 6.9 \mathrm{~Hz}, 3 \mathrm{H})$, 0.78-0.89 (m, $1 \mathrm{H}), 1.04-1.13(\mathrm{~m}, 2 \mathrm{H}), 1.14-1.25(\mathrm{~m}, 1 \mathrm{H}), 1.38-1.46(\mathrm{~m}, 1 \mathrm{H}), 1.49(\mathrm{~s}, 9 \mathrm{H}), 1.49-$ $1.53(\mathrm{~m}, 2 \mathrm{H}), 2.24$ (ddd, J 13.3, 8.0, $5.9 \mathrm{~Hz}, 1 \mathrm{H}), 2.43(\mathrm{dt}, J$ 13.3, $8.0 \mathrm{~Hz}, 1 \mathrm{H}), 3.29$ (td, J 8.0, 5.9 $\mathrm{Hz}, 1 \mathrm{H}), 3.81(\mathrm{t}, J 8.3 \mathrm{~Hz}, 1 \mathrm{H}), 4.34(\mathrm{td}, J 10.9,4.4 \mathrm{~Hz}, 1 \mathrm{H}), 4.44(\mathrm{~d}, J 7.8 \mathrm{~Hz}, 1 \mathrm{H}), 7.17-7.21(\mathrm{~m}$, $1 \mathrm{H}), 7.24-7.28(\mathrm{~m}, 2 \mathrm{H}), 7.30-7.33(\mathrm{~m}, 2 \mathrm{H})$.

${ }^{13} \mathrm{C}$ NMR $\left(100 \mathrm{MHz}, \mathrm{CDCl}_{3}, 293 \mathrm{~K}\right): \delta 15.99,20.75,21.75,23.10,25.83,28.07$ (3C), 31.03, 34.01, $34.75,39.79,46.55,49.38,60.66,65.74,73.99,81.39,127.09$ (2C), 127.31, 128.22 (2C), 139.31, $172.30,172.47$.

[1] Barr, D. A.; Dorrity, M. J.; Grigg, R.; Hargreaves, S.; Malone, J. F.; Montgomery, J.; Redpath, J.; Stevenson, P.; Thornton-Pett, M. Tetrahedron, 1995, 51, 273-294.

[2] Jovanovic, P.; Randelovic, J.; Ivkovic, B.; Suteu, C.; Tokic Vujosevic, Z.; Savic, V. J. Serb. Chem. Soc. 2014, 79, 767-778. 
4-((1S,2R,5S)-2-isopropyl-5-methylcyclohexyl) $\quad$ 2-methyl $\quad(2 R, 4 R, 5 S)$-5-phenylpyrrolidine-2,4dicarboxylate D-1a

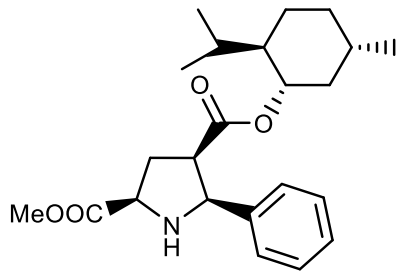

Yield $73 \%, 11.000$ g, colorless crystals, m.p. $103-105{ }^{\circ} \mathrm{C},[\alpha]_{D}^{33}-8.3^{\circ}(c$ 1.13, $\mathrm{MeOH})$.

${ }^{1} \mathrm{H}$ NMR $\left(400 \mathrm{MHz}, \mathrm{CDCl}_{3}, 293 \mathrm{~K}\right): \delta 0.17-0.26(\mathrm{~m}, 1 \mathrm{H}), 0.53(\mathrm{~d}, J 6.9 \mathrm{~Hz}$, $3 \mathrm{H}), 0.62-0.70(\mathrm{~m}, 1 \mathrm{H}), 0.70(\mathrm{~d}, J 6.9 \mathrm{~Hz}, 3 \mathrm{H}), 0.75(\mathrm{~d}, J 6.9 \mathrm{~Hz}, 3 \mathrm{H})$, 0.79-0.89 $(\mathrm{m}, 1 \mathrm{H}), 1.03-1.22(\mathrm{~m}, 3 \mathrm{H}), 1.42-1.53(\mathrm{~m}, 3 \mathrm{H}), 2.34$ (ddd, J 13.0, 8.0, $5.4 \mathrm{~Hz}, 1 \mathrm{H}), 2.46(\mathrm{dt}, J 13.0,8.0 \mathrm{~Hz}, 1 \mathrm{H}), 3.30$ (td, J 7.6, 5.4 $\mathrm{Hz}, 1 \mathrm{H}), 3.79(\mathrm{~s}, 3 \mathrm{H}), 3.94-3.98(\mathrm{~m}, 1 \mathrm{H}), 4.31(\mathrm{td}, J 10.8,4.3 \mathrm{~Hz}, 1 \mathrm{H}), 4.47(\mathrm{~d}, J 7.6 \mathrm{~Hz}, 1 \mathrm{H}), 7.18-$ $7.22(\mathrm{~m}, 1 \mathrm{H}), 7.24-7.29(\mathrm{~m}, 2 \mathrm{H}), 7.32-7.33(\mathrm{~m}, 2 \mathrm{H})$.

${ }^{13} \mathrm{C}$ NMR $\left(100 \mathrm{MHz}, \mathrm{DMSO}-\mathrm{d}_{6}, 293 \mathrm{~K}\right): \delta$ 16.00, 20.48, 21.67, 22.72, 25.45, 30.47, 32.94, 33.55, $39.36,46.01,48.36,51.74,58.77,64.22,72.86,126.91,127.23$ (2C), 127.74 (2C), 140.18, $171.86,173.47$.

Anal. Calcd for $\mathrm{C}_{23} \mathrm{H}_{33} \mathrm{NO}_{4}$ : C 71.29, H 8.58, N 3.61. Found: C 71.42, H 8.65, N 3.49.

4-((1R,2S,5R)-2-isopropyl-5-methylcyclohexyl) 2-methyl

$(2 S, 4 S, 5 R)-5-(3-$ chlorophenyl)pyrrolidine-2,4-dicarboxylate L-1d

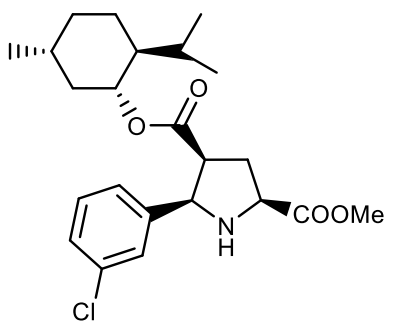

Yield $44 \%, 1.250 \mathrm{~g}$, colorless crystals, m.p. $69-72{ }^{\circ} \mathrm{C},[\alpha]_{D^{20}}+0.7^{\circ}$ (c 1.13, $\mathrm{MeOH})$.

${ }^{1} \mathrm{H}$ NMR (400 MHz, CDCl, $\left.293 \mathrm{~K}\right): \delta 0.34$ (q, 1H, J $12.0 \mathrm{~Hz}$ ), 0.57 (d, J 6.9 $\mathrm{Hz}, 3 \mathrm{H}), 0.68-0.78(\mathrm{~m}, 1 \mathrm{H}), 0.76(\mathrm{~d}, J 6.9 \mathrm{~Hz}, 3 \mathrm{H}), 0.79(\mathrm{~d}, J 6.9 \mathrm{~Hz}, 3 \mathrm{H})$, 0.81-0.92 $(\mathrm{m}, 1 \mathrm{H}), 1.12-1.27(\mathrm{~m}, 3 \mathrm{H}), 1.49-1.57(\mathrm{~m}, 3 \mathrm{H}), 2.36$ (ddd, J 13.2, 8.0, $5.8 \mathrm{~Hz}, 1 \mathrm{H}$ ), 2.46 (dt, J 13.2, $8.0 \mathrm{~Hz}, 1 \mathrm{H}$ ), 3.30 (td, J 8.0, $5.4 \mathrm{~Hz}$, $1 \mathrm{H}), 3.80(\mathrm{~s}, 3 \mathrm{H}), 3.97(\mathrm{t}, J 8.0 \mathrm{~Hz}, 1 \mathrm{H}), 4.37(\mathrm{td}, J 10.8,4.3 \mathrm{~Hz}, 1 \mathrm{H}), 4.46$ (d, J 8.0 Hz, 1H), 7.18-7.27 (m, 3H), $7.33(\mathrm{~s}, 1 \mathrm{H})$.

${ }^{13} \mathrm{C}$ NMR $\left(100 \mathrm{MHz}, \mathrm{CDCl}_{3}, 293 \mathrm{~K}\right): \delta 16.12,20.72,21.77,23.24,26.10,31.09,34.03,39.92$, $46.61,49.04,52.29,59.75,65.06,74.50,125.35,127.55,127.64,129.58,134.20,141.30$, $172.03,173.41$.

Anal. Calcd for $\mathrm{C}_{23} \mathrm{H}_{32} \mathrm{ClNO}_{4}$ : C 65.47, H 7.64, N 3.32. Found: C 65.19, H 7.58, N 3.25.

4-((1S,2R,5S)-2-isopropyl-5-methylcyclohexyl)

2-methyl

$(2 R, 4 R, 5 S)-5-(3-$ chlorophenyl)pyrrolidine-2,4-dicarboxylate D-1d

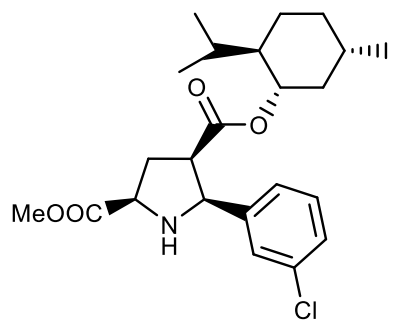

Yield $61 \%, 1.340 \mathrm{~g}$, colorless crystals, m.p. $69-72{ }^{\circ} \mathrm{C},[\alpha]_{D^{20}}-1.2^{\circ}$ (c 1.13, $\mathrm{MeOH})$.

${ }^{1} \mathrm{H}$ NMR $\left(400 \mathrm{MHz}, \mathrm{CDCl}_{3}, 293 \mathrm{~K}\right): \delta 0.34$ (q, 1H, J $\left.12.0 \mathrm{~Hz}\right), 0.57$ (d, J 6.9 $\mathrm{Hz}, 3 \mathrm{H}), 0.68-0.78(\mathrm{~m}, 1 \mathrm{H}), 0.76(\mathrm{~d}, J 6.9 \mathrm{~Hz}, 3 \mathrm{H}), 0.79(\mathrm{~d}, J 6.9 \mathrm{~Hz}, 3 \mathrm{H})$, 0.81-0.92 (m, 1H), 1.12-1.27 (m, 3H), 1.49-1.57 (m, 3H), 2.36 (ddd, J 13.2, 8.0, $5.8 \mathrm{~Hz}, 1 \mathrm{H}$ ), 2.46 (dt, J 13.2, $8.0 \mathrm{~Hz}, 1 \mathrm{H}$ ), 3.30 (td, J 8.0, $5.4 \mathrm{~Hz}$, $1 \mathrm{H}), 3.80(\mathrm{~s}, 3 \mathrm{H}), 3.97(\mathrm{t}, J 8.0 \mathrm{~Hz}, 1 \mathrm{H}), 4.37(\mathrm{td}, J 10.8,4.3 \mathrm{~Hz}, 1 \mathrm{H}), 4.46$ (d, J $8.0 \mathrm{~Hz}, 1 \mathrm{H}), 7.18-7.27(\mathrm{~m}, 3 \mathrm{H}), 7.33(\mathrm{~s}, 1 \mathrm{H})$.

${ }^{13} \mathrm{C}$ NMR $\left(100 \mathrm{MHz}, \mathrm{CDCl}_{3}, 293 \mathrm{~K}\right): \delta 16.12,20.72,21.77,23.24,26.10,31.09,34.03,39.92$, 46.61 , 49.04, 52.29, 59.75, 65.06, 74.50, 125.35, 127.55, 127.64, 129.58, 134.20, 141.30, $172.03,173.41$.

Anal. Calcd for $\mathrm{C}_{23} \mathrm{H}_{32} \mathrm{ClNO}_{4}$ : C 65.47, H 7.64, N 3.32. Found: C 65.68, H 7.30, N 3.39. 


\section{General procedure of cycloadditive oligomerization}

\section{Synthesis of acrylamides L-n_A and D-n_A from monomers L-1 and D-1 and oligomers L-n and D-n}

TEA (1.16 ml, $0.84 \mathrm{~g}, 8.4 \mathrm{mmol})$ was added to a stirred solution of corresponding monomer or oligomer $(5.2 \mathrm{mmol})$ in $70 \mathrm{ml}$ of $\mathrm{CH}_{2} \mathrm{Cl}_{2}$ at $0{ }^{\circ} \mathrm{C}$. Then acryloyl chloride $(0.62 \mathrm{ml}, 0.70 \mathrm{~g}, 7.8$ $\mathrm{mmol}$ ) was added dropwise to the reaction mixture under inert atmosphere. Cooling bath was removed after $15 \mathrm{~min}$ of completion of acryloyl chloride addition. The reaction mixture was stirred at $\mathrm{rt}$ for additional $24 \mathrm{~h}$, then washed with $\mathrm{H}_{2} \mathrm{O}(40 \mathrm{~mL})$, brine $(40 \mathrm{~mL})$, dried over $\mathrm{Na}_{2} \mathrm{SO}_{4}$ and concentrated under reduced pressure. Acrylamides were isolated by column chromatography on silica gel using hexane/AcOEt from 3:1 to 1:2 as an eluent.

\section{Synthesis of oligomers $L-(n+1)$ and $D-(n+1)$ from acrylamides $L-n \_A$ and $D-n \_A$}

A solution of TEA $(0.39 \mathrm{ml}, 0.28 \mathrm{~g}, 2.8 \mathrm{mmol})$ in $5 \mathrm{ml}$ of toluene was added dropwise to the stirred mixture of corresponding iminoester $\mathrm{ArCH}=\mathrm{NCH}_{2} \mathrm{COOR}(2.2 \mathrm{mmol}), \mathrm{AgOAc}(0.50 \mathrm{~g}, 2.8$ mmol), acrylamide L-n_A or D-n_A $(1.9 \mathrm{mmol})$ in $40 \mathrm{ml}$ of dry toluene under inert atmosphere. Reaction flask was protected from the light with aluminium foil. The reaction mixture was stirred at room temperature for 12-48 $\mathrm{h}$. Then toluene was removed by evaporation under reduced pressure. The solid residue was suspended in $50 \mathrm{ml} \mathrm{CH} \mathrm{Cl}_{2}$ and the precipitate was removed by filtration. Organic phase was washed with $25 \mathrm{ml}$ of water, $25 \mathrm{ml}$ of brine and dried over $\mathrm{Na}_{2} \mathrm{SO}_{4}$, filtered and volatiles were evaporated under reduced pressure. The products were isolated by column chromatography on silica gel using hexane/AcOEt from 3:1 to 1:2 as an eluent.

4-((1R,2S,5R)-2-isopropyl-5-methylcyclohexyl)

2-methyl

$(2 S, 4 S, 5 R)-1$-acryloyl-5phenylpyrrolidine-2,4-dicarboxylate L-1a_A

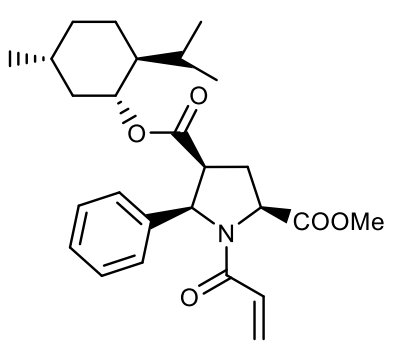

Yield $89 \%, 4.100 \mathrm{~g}$, colorless crystals, m.p. $61-63{ }^{\circ} \mathrm{C},[\alpha]_{D}{ }^{21}-71.0^{\circ}$ (c $0.925, \mathrm{MeOH})$.

${ }^{1} \mathrm{H}$ NMR $\left(400 \mathrm{MHz}, \mathrm{CDCl}_{3}, 293 \mathrm{~K}\right): \delta 0.48(\mathrm{~d}, J 6.9 \mathrm{~Hz}, 3 \mathrm{H}), 0.74$ (d, J 6.9 $\mathrm{Hz}, 3 \mathrm{H}), 0.74-0.82(\mathrm{~m}, 2 \mathrm{H}), 0.82(\mathrm{~d}, J 6.9 \mathrm{~Hz}, 3 \mathrm{H}), 0.86-0.95(\mathrm{~m}, 1 \mathrm{H})$, 1.18-1.34 (m, 3H), 1.55-1.61 (m, 3H), 2.36-2.43 (m, 1H), 2.49-2.58 (m, $1 \mathrm{H}), 3.43-3.53(\mathrm{~m}, 1 \mathrm{H}), 3.85(\mathrm{~s}, 3 \mathrm{H}), 4.39-4.49(\mathrm{~m}, 2 \mathrm{H}), 5.30(\mathrm{~d}, J 8.4 \mathrm{~Hz}$, $1 \mathrm{H}), 5.52$ (dd, J 10.3, 1.6 Hz, 1H), 6.06 (dd, J 16.6, $10.3 \mathrm{~Hz}, 1 \mathrm{H}), 6.31$ (dd, J 16.6, 1.6 Hz, 1H), 7.25-7.33 (m, 3H), 7.63 (d, J 7.0 Hz, 2H).

${ }^{13} \mathrm{C}$ NMR (100 MHz, DMSO-d6, $\left.293 \mathrm{~K}\right): \delta$ 16.08, 20.54, 21.77, 22.74, 25.31, 28.55, 30.61, 33.51, $38.87,46.10,48.50,51.97,58.66,61.82,73.92,127.87,127.97$ (2C), 128.12 (2C), 128.29, $128.36,139.20,164.07,168.24,172.04$

Anal. Calcd for $\mathrm{C}_{26} \mathrm{H}_{35} \mathrm{NO}_{5}$ : C 70.72, H 7.99, N 3.17. Found: C 70.58, H 7.72, N 3.17.

4-((1R,2S,5R)-2-isopropyl-5-methylcyclohexyl) $\quad$ 2-methyl $\quad(2 S, 4 S, 5 R)$-1-acryloyl-5-(4bromophenyl)pyrrolidine-2,4-dicarboxylate L-1b_A

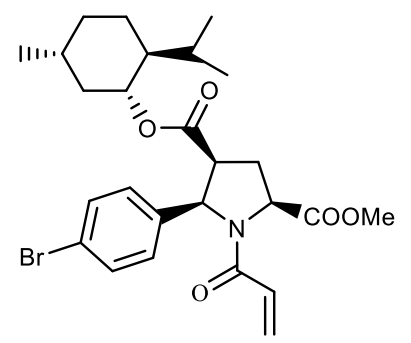

Yield $90 \%, 5.800 \mathrm{~g}$, colorless crystals, m.p. $70-72{ }^{\circ} \mathrm{C},[\alpha]_{D^{20}}-32.0^{\circ}(\mathrm{c}$ $1.13, \mathrm{MeOH})$. 
${ }^{1} \mathrm{H}$ NMR (400 MHz, DMSO-d $\left.6,293 \mathrm{~K}\right): \delta 0.48(\mathrm{~d}, J 7.0 \mathrm{~Hz}, 3 \mathrm{H}), 0.71-0.77(\mathrm{~m}, 1 \mathrm{H}), 0.74(\mathrm{~d}, 3 \mathrm{H}, J 7.0$ $\mathrm{Hz}, 3 \mathrm{H}), 0.81(\mathrm{~d}, J 7.0 \mathrm{~Hz}, 3 \mathrm{H}), 0.84-0.93(\mathrm{~m}, 1 \mathrm{H}), 1.15-1.40(\mathrm{~m}, 4 \mathrm{H}), 1.50-1.58(\mathrm{~m}, 2 \mathrm{H}), 2.15-2.25$ (m, 1H), 2.15-2.25 (m, 1H), 2.35-2.41 (m, 1H), $3.72(\mathrm{~s}, 3 \mathrm{H}), 3.72-3.79(\mathrm{~m}, 1 \mathrm{H}), 4.32(\mathrm{td}, J 11.0,4.3$ $\mathrm{Hz}, 1 \mathrm{H}), 4.32$ (dd, J 11.0, $7.0 \mathrm{~Hz}, 1 \mathrm{H}), 5.49$ (d, J $9.0 \mathrm{~Hz}, 1 \mathrm{H}$ ), 5.55 (dd, J 10.0, $2.6 \mathrm{~Hz}, 1 \mathrm{H}$ ), 6.06 (dd, J 16.6, $2.6 \mathrm{~Hz}, 1 \mathrm{H}), 6.14$ (dd, J 16.6, $10.0 \mathrm{~Hz}, 1 \mathrm{H}), 7.50-7.57(\mathrm{~m}, 4 \mathrm{H})$.

${ }^{13} \mathrm{C}$ NMR $\left(100 \mathrm{MHz}, \mathrm{CDCl}_{3}, 293 \mathrm{~K}\right): \delta$ 16.01, 20.70, 21.91, 23.05, 25.81, 28.65, 31.19, 33.96, 40.76, 46.67, 49.93, 52.48, 59.14, 62.31, 75.36, 122.66, 127.36, 129.71, 129.84 (2C), 131.79 (2C), 137.23, 165.27, 167.93, 172.03 .

Anal. Calcd for $\mathrm{C}_{26} \mathrm{H}_{34} \mathrm{BrNO}_{5}: 60.00, \mathrm{H} 6.58$, N 2.69. Found: 60.25, H 6.53, N 2.60.

2-(tert-butyl) 4-((1R,2S,5R)-2-isopropyl-5-methylcyclohexyl)

(2S,4S,5R)-1-acryloyl-5phenylpyrrolidine-2,4-dicarboxylate L-1c_A

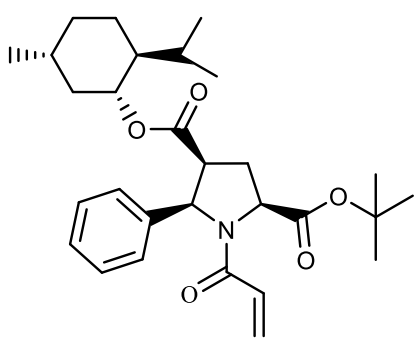

Yield $95 \%, 8.800 \mathrm{~g}$, yellowish crystals, m.p. $64-66^{\circ} \mathrm{C},[\alpha]_{D}{ }^{20}-60.7^{\circ}(\mathrm{c}$ 1.13, $\mathrm{MeOH})$.

${ }^{1} \mathrm{H}$ NMR $\left(400 \mathrm{MHz}, \mathrm{CDCl}_{3}, 293 \mathrm{~K}\right): \delta 0.52(\mathrm{~d}, J 7.0 \mathrm{~Hz}, 3 \mathrm{H}), 0.76(\mathrm{~d}, J 7.0$ $\mathrm{Hz}, 3 \mathrm{H}), 0.79-0.84(\mathrm{~m}, 1 \mathrm{H}), 0.84(\mathrm{~d}, J 7.0 \mathrm{~Hz}, 3 \mathrm{H}), 0.86-0.95(\mathrm{~m}, 1 \mathrm{H})$, 1.22-1.38 (m, 3H), 1.50-1.59 (m, 3H), $1.54(\mathrm{~s}, 9 \mathrm{H}), 2.34-2.41(\mathrm{~m}, 1 \mathrm{H})$, 2.45-2.54 (m, 1H), 3.42-3.49 (m, 1H), $4.33(\mathrm{dd}, J 11.6,6.6 \mathrm{~Hz}, 1 \mathrm{H})$, 4.41-4.48 (m, 1H), $5.26(\mathrm{~d}, J 8.7 \mathrm{~Hz}, 1 \mathrm{H}), 5.49(\mathrm{dd}, J 10.4,1.8 \mathrm{~Hz}, 1 \mathrm{H})$, 6.04 (dd, J 16.8, 10.4 Hz, 1H), 6.29 (dd, J 16.8, 1.8 Hz, 1H), 7.25-7.33 (m, 3H), 7.61-7.64 (m, 2H). ${ }^{13} \mathrm{C}$ NMR $\left(100 \mathrm{MHz} \mathrm{CDCl}_{3}, 293 \mathrm{~K}\right): \delta 16.18,20.75,21.90,23.21,25.86,28.05(3 \mathrm{C}), 28.73,31.22$, $34.03,40.73,46.79,49.66,60.25,63.06,75.12,81.67,128.13$ (3C), 128.29, 128.47 (2C), 128.79, $138.30,165.43,168.28,170.25$.

Anal. Calcd for $\mathrm{C}_{29} \mathrm{H}_{41} \mathrm{NO}_{5}$ : C 72.02, H 8.55, N 2.90. Found: 71.78, H 8.34, N 2.74.

4-((1S,2R,5S)-2-isopropyl-5-methylcyclohexyl)

2-methyl

$(2 R, 4 R, 5 S)-1$-acryloyl-5phenylpyrrolidine-2,4-dicarboxylate D-1a_A

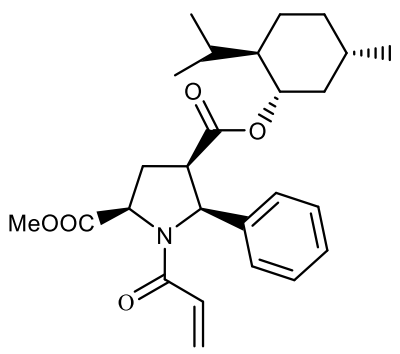

Yield $91 \%, 10.400 \mathrm{~g}$, yellowish crystals, m.p. $61-62^{\circ} \mathrm{C},[\alpha]_{D}{ }^{21}+71.4^{\circ}(c$ $1.05, \mathrm{MeOH})$.

${ }^{1} \mathrm{H}$ NMR $\left(400 \mathrm{MHz}, \mathrm{DMSO}-\mathrm{d}_{6}, 293 \mathrm{~K}\right): \delta 0.49(\mathrm{~d}, J 7.0 \mathrm{~Hz}, 3 \mathrm{H}), 0.74(\mathrm{~d}, J$ $7.0 \mathrm{~Hz}, 3 \mathrm{H}), 0.75-0.80(\mathrm{~m}, 2 \mathrm{H}), 0.80(\mathrm{~d}, J 7.0 \mathrm{~Hz}, 3 \mathrm{H}), 0.85-0.95(\mathrm{~m}, 1 \mathrm{H})$, 1.17-1.32 (m, 2H), 1.17-1.32 (m, 2H), 1.34-1.46 (m, 2H), 1.52-1.58 (m, $2 \mathrm{H}), 2.20-2.29(\mathrm{~m}, 1 \mathrm{H}), 2.33-2.39(\mathrm{~m}, 1 \mathrm{H}), 3.72(\mathrm{~s}, 3 \mathrm{H}), 3.75-3.78(\mathrm{~m}$, $1 \mathrm{H}), 4.27-4.34(\mathrm{~m}, 1 \mathrm{H}), 4.37-4.41(\mathrm{~m}, 1 \mathrm{H}), 5.45(\mathrm{~d}, J 8.6 \mathrm{~Hz}, 1 \mathrm{H}), 5.53$ (dd, J 9.8, 2.6 Hz, 1H), 6.05 (dd, J 16.7, $2.6 \mathrm{~Hz}, 1 \mathrm{H}$ ), 6.13 (dd, J 16.7, 9.8 $\mathrm{Hz}, 1 \mathrm{H}), 7.27-7.33(\mathrm{~m}, 3 \mathrm{H}), 7.63(\mathrm{~d}, \mathrm{~J} 7.0 \mathrm{~Hz}, 2 \mathrm{H})$.

${ }^{13} \mathrm{C}$ NMR $(100 \mathrm{MHz}$, DMSO-d $6,293 \mathrm{~K}): \delta 16.08,20.54,21.77,22.74,25.31,28.55,30.61,33.51$, $38.87,46.10,48.50,51.97,58.66,61.82,73.92,127.87,127.97$ (2C), 128.12 (2C), 128.29, $128.36,139.20,164.07,168.24,172.04$.

Anal. Calcd for $\mathrm{C}_{26} \mathrm{H}_{35} \mathrm{NO}_{5}$ : C 70.72, H 7.99, N 3.17. Found: C 70.72, H 7.69, N 2.87.

4-((1R,2S,5R)-2-isopropyl-5-methylcyclohexyl) 2-methyl

$(2 S, 4 S, 5 R)-1$-acryloyl-5-(3chlorophenyl)pyrrolidine-2,4-dicarboxylate L-1d_A

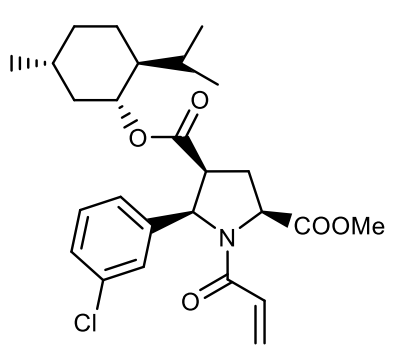

Yield $82 \%, 1.150 \mathrm{~g}$, colorless crystals, m.p. $84-86{ }^{\circ} \mathrm{C},[\alpha]_{D}{ }^{20}+52.3^{\circ}(\mathrm{c}$ $1.13, \mathrm{MeOH})$.

${ }^{1} \mathrm{H}$ NMR $\left(400 \mathrm{MHz}, \mathrm{DMSO}-\mathrm{d}_{6}, 293 \mathrm{~K}\right): \delta 0.52(\mathrm{~d}, J 7.0 \mathrm{~Hz}, 3 \mathrm{H}), 0.77$ (d, J $7.0 \mathrm{~Hz}, 3 \mathrm{H}), 0.78-0.84(\mathrm{~m}, 2 \mathrm{H}), 0.83(\mathrm{~d}, J 7.0 \mathrm{~Hz}, 3 \mathrm{H}), 0.88-0.96(\mathrm{~m}, 1 \mathrm{H})$, 
1.23-1.31 (m, 2H), 1.43-1.48 (m, 2H), 1.53-1.60 (m, 2H), 2.15-2.24 (m, 1H), 2.34-2.41 (m, $1 \mathrm{H})$, $3.73(\mathrm{~s}, 3 \mathrm{H}), 3.74-3.80(\mathrm{~m}, 1 \mathrm{H}), 4.31-4.41(\mathrm{~m}, 2 \mathrm{H}), 5.53(\mathrm{~d}, J 8.8 \mathrm{~Hz}, 1 \mathrm{H}), 5.56(\mathrm{dd}, J 9.8,2.6 \mathrm{~Hz}$, $1 \mathrm{H}), 6.07$ (dd, J 16.6, $2.6 \mathrm{~Hz}, 1 \mathrm{H}), 6.15(\mathrm{dd}, J 16.6,9.8 \mathrm{~Hz}, 1 \mathrm{H}), 7.31-7.36(\mathrm{~m}, 2 \mathrm{H}), 7.62-7.66(\mathrm{~m}$, $2 \mathrm{H})$.

${ }^{13} \mathrm{C}$ NMR (100 MHz, DMSO-d, $\left.293 \mathrm{~K}\right): \delta 16.04,20.47,21.75,22.73,25.41,28.49,30.59,33.51$, $40.18,46.11,48.38,51.97,58.66,61.01,74.06,126.47,127.87,127.89,128.11,128.62,130.02$, $132.86,141.70,164.01,168.10,172.09$.

Anal. Calcd for $\mathrm{C}_{26} \mathrm{H}_{34} \mathrm{ClNO}_{5}$ : C 65.60, H 7.20, N 2.94. Found: C 65.81, H 7.17, N 2.79.

4-((1S,2R,5S)-2-isopropyl-5-methylcyclohexyl)

2-methyl

$(2 R, 4 R, 5 S)-1$-acryloyl-5-(3-

chlorophenyl)pyrrolidine-2,4-dicarboxylate D-1d_A

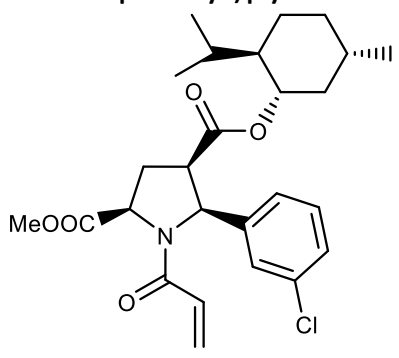

Yield $76 \%, 1.280 \mathrm{~g}$, colorless crystals, m.p. $84-86{ }^{\circ} \mathrm{C},[\alpha]_{D}{ }^{20}-46.2^{\circ}(\mathrm{c}$ $1.07, \mathrm{MeOH})$.

${ }^{1} \mathrm{H}$ NMR $\left(400 \mathrm{MHz}, \mathrm{DMSO}-\mathrm{d}_{6}, 293 \mathrm{~K}\right): \delta 0.52(\mathrm{~d}, J 7.0 \mathrm{~Hz}, 3 \mathrm{H}), 0.77$ (d, J $7.0 \mathrm{~Hz}, 3 \mathrm{H}), 0.78-0.84(\mathrm{~m}, 2 \mathrm{H}), 0.83(\mathrm{~d}, J 7.0 \mathrm{~Hz}, 3 \mathrm{H}), 0.88-0.96(\mathrm{~m}, 1 \mathrm{H})$, 1.23-1.31 (m, 2H), 1.43-1.48 (m, 2H), 1.53-1.60 (m, 2H), 2.15-2.24 (m, $1 \mathrm{H}), 2.34-2.41(\mathrm{~m}, 1 \mathrm{H}), 3.73(\mathrm{~s}, 3 \mathrm{H}), 3.74-3.80(\mathrm{~m}, 1 \mathrm{H}), 4.31-4.41(\mathrm{~m}$, $2 \mathrm{H}), 5.53(\mathrm{~d}, J 8.8 \mathrm{~Hz}, 1 \mathrm{H}), 5.56(\mathrm{dd}, J 9.8,2.6 \mathrm{~Hz}, 1 \mathrm{H}), 6.07(\mathrm{dd}, J$ 16.6, 2.6 Hz, 1H), 6.15 (dd, J 16.6, 9.8 Hz, 1H), 7.31-7.36 (m, 2H), 7.62-7.66 (m, 2H).

${ }^{13} \mathrm{C}$ NMR (100 MHz, DMSO-d6, $\left.293 \mathrm{~K}\right): \delta$ 16.04, 20.47, 21.75, 22.73, 25.41, 28.49, 30.59, 33.51, $40.18,46.11,48.38,51.97,58.66,61.01,74.06,126.47,127.87,127.89,128.11,128.62,130.02$, $132.86,141.70,164.01,168.10,172.09$.

Anal. Calcd for $\mathrm{C}_{26} \mathrm{H}_{34} \mathrm{ClNO}_{5}$ : C 65.60, H 7.20, N 2.94. Found: C 65.91, H 7.25, N 2.85.

4-((1R,2S,5R)-2-isopropyl-5-methylcyclohexyl) $\quad$ 2-methyl $\quad(2 S, 4 S, 5 R)-1-((2 S, 3 R, 5 R)-5-$ (methoxycarbonyl)-2-phenylpyrrolidine-3-carbonyl)-5-phenylpyrrolidine-2,4-dicarboxylate L-2a

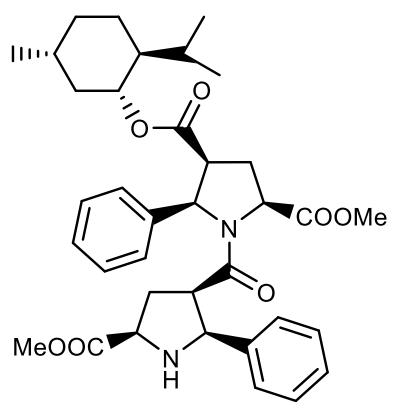

Yield $76 \%, 3.000 \mathrm{~g}$, colorless crystals, m.p. $96-98{ }^{\circ} \mathrm{C},[\alpha]_{D^{21}}-39.3^{\circ}(\mathrm{c}$ $0.915, \mathrm{MeOH})$.

${ }^{1} \mathrm{H}$ NMR $\left(400 \mathrm{MHz}, \mathrm{DMSO}-\mathrm{d}_{6}, 293 \mathrm{~K}\right): \delta 0.51(\mathrm{~d}, J 6.8 \mathrm{~Hz}, 3 \mathrm{H}), 0.70-0.77$ $(\mathrm{m}, 1 \mathrm{H}), 0.80(\mathrm{~d}, J 6.8 \mathrm{~Hz}, 3 \mathrm{H}), 0.82(\mathrm{~d}, J 6.8 \mathrm{~Hz}, 3 \mathrm{H}), 0.85-0.95(\mathrm{~m}, 1 \mathrm{H})$, $1.21-1.29(\mathrm{~m}, 2 \mathrm{H}), 1.31-1.36(\mathrm{~m}, 1 \mathrm{H}), 1.39-1.48(\mathrm{~m}, 1 \mathrm{H}), 1.54-1.60(\mathrm{~m}$, $2 \mathrm{H}), 1.85-1.97(\mathrm{~m}, 2 \mathrm{H}), 1.99-2.08(\mathrm{~m}, 2 \mathrm{H}), 2.27(\mathrm{ddd}, J 13.0,8.2,6.0 \mathrm{~Hz}$, $1 \mathrm{H}), 2.88-2.95(\mathrm{~m}, 2 \mathrm{H}), 3.65(\mathrm{~s}, 3 \mathrm{H}), 3.66(\mathrm{~s}, 3 \mathrm{H}), 3.66-3.70(\mathrm{~m}, 2 \mathrm{H})$, $4.23(\mathrm{dd}, J 10.8,4.2 \mathrm{~Hz}, 1 \mathrm{H}), 4.37-4.40(\mathrm{~m}, 1 \mathrm{H}), 4.61(\mathrm{~d}, J 8.4 \mathrm{~Hz}, 1 \mathrm{H})$, 7.27-7.32 (m, $4 \mathrm{H}), 7.38-7.44(\mathrm{~m}, 4 \mathrm{H}), 7.48-7.51(\mathrm{~m}, 2 \mathrm{H})$.

${ }^{13} \mathrm{C}$ NMR (100 MHz, DMSO-d $\left.6,293 \mathrm{~K}\right): \delta$ 16.17, 20.52, 21.74, 22.80, 25.43, 27.73, 30.55, 33.15, $33.50,40.08,46.19,46.46,48.20,51.66,51.87,58.21,58.88,61.65,65.41,73.91,127.29$ (2C), $127.62,128.00$ (3C), 128.08 (2C), 128.23 (2C), 137.97, 139.23, 167.58, 171.65 (2C), 173.20. Anal. Calcd for $\mathrm{C}_{36} \mathrm{H}_{46} \mathrm{~N}_{2} \mathrm{O}_{7}$ : C 69.88, H 7.49, N 4.53. Found: $\mathrm{C} 70.14, \mathrm{H} 7.51, \mathrm{~N} 4.58$.

4-((1R,2S,5R)-2-isopropyl-5-methylcyclohexyl) 2-methyl $\quad(2 S, 4 S, 5 R)-5$-(4-bromophenyl)-1((2S,3R,5R)-2-(4-bromophenyl)-5-(methoxycarbonyl)pyrrolidine-3-carbonyl)pyrrolidine-2,4dicarboxylate $\mathbf{L}-\mathbf{2} \mathbf{b}$

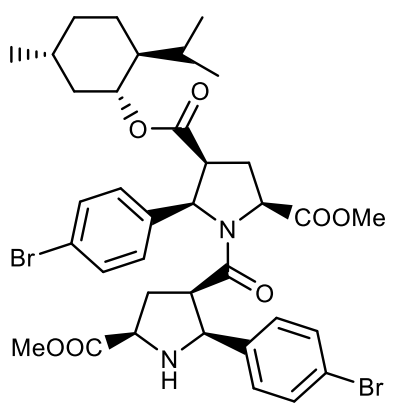

Yield $79 \%, 6.000 \mathrm{~g}$, colorless crystals, m.p. $110-108{ }^{\circ} \mathrm{C},[\alpha]_{D}{ }^{20}+2.6^{\circ}(\mathrm{c}$ 1.13, $\mathrm{MeOH})$.

${ }^{1} \mathrm{H} \mathrm{NMR}\left(400 \mathrm{MHz}, \mathrm{CDCl}_{3}, 293 \mathrm{~K}\right): \delta 0.50(\mathrm{~d}, J 6.8 \mathrm{~Hz}, 3 \mathrm{H}), 0.67-0.78(\mathrm{~m}$, $2 \mathrm{H}), 0.80(\mathrm{~d}, J 6.8 \mathrm{~Hz}, 3 \mathrm{H}), 0.84(\mathrm{~d}, J 6.8 \mathrm{~Hz}, 3 \mathrm{H}), 0.84-0.95(\mathrm{~m}, 1 \mathrm{H})$, 
1.17-1.37 (m, 3H), 1.52-1.63 (m, 3H), 2.01-2.20 (m, 3H), 2.27-2.40 (m, 2H), 2.86-2.91 (m, $1 \mathrm{H})$, $3.74(\mathrm{~s}, 3 \mathrm{H}), 3.74-3.78(\mathrm{~m}, 1 \mathrm{H}), 3.76(\mathrm{~s}, 3 \mathrm{H}), 3.83(\mathrm{dd}, J 10.6,7.0 \mathrm{~Hz}, 1 \mathrm{H}), 4.25-4.31(\mathrm{~m}, 1 \mathrm{H}), 4.38$ (td, J 10.8, 4.2 Hz, 1H), 4.49 (d, J $8.2 \mathrm{~Hz}, 1 \mathrm{H}), 7.30$ (d, J $8.3 \mathrm{~Hz} 4 \mathrm{H}), 7.41(\mathrm{~s}, 4 \mathrm{H}), 7.53$ (d, J $8.3 \mathrm{~Hz}$, $2 \mathrm{H})$.

${ }^{13} \mathrm{C}$ NMR $\left(100 \mathrm{MHz}, \mathrm{CDCl}_{3}, 293 \mathrm{~K}\right): \delta 15.95,20.84,21.90,22.99,25.76,28.16,31.14,33.95$, $34.11,40.72,46.78,47.43,49.16,52.21,52.36,58.63,59.94,61.97,66.29,75.22,122.22$, 122.69, 129.12 (2C), 129.77 (2C), 131.72 (2C), 131.75 (2C), 136.44, 137.34, 167.64, 171.82, $171.87,173.15$.

HRMS (FAB) calculated for $\mathrm{C}_{36} \mathrm{H}_{44} \mathrm{O}_{7} \mathrm{~N}_{2}{ }^{79} \mathrm{Br}_{2} 774.1510$, found 774.1510 .

2-(tert-butyl) $\quad 4-((1 R, 2 S, 5 R)$-2-isopropyl-5-methylcyclohexyl) $\quad(2 S, 4 S, 5 R)-1-((2 S, 3 R, 5 R)-5$-(tertbutoxycarbonyl)-2-phenylpyrrolidine-3-carbonyl)-5-phenylpyrrolidine-2,4-dicarboxylate L-2C

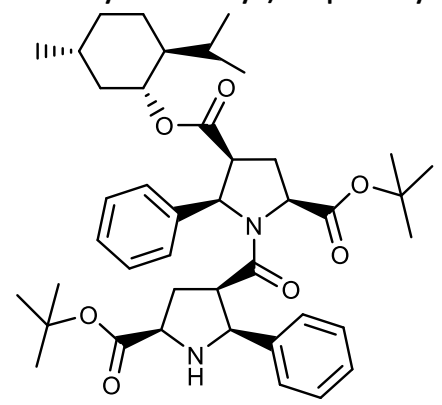

Yield $80 \%, 9.900 \mathrm{~g}$, colorless crystals, m.p. $86-88^{\circ} \mathrm{C},[\alpha]_{D}{ }^{21}-36.9^{\circ}$ (c $0.83, \mathrm{MeOH})$.

${ }^{1} \mathrm{H}$ NMR $\left(400 \mathrm{MHz}, \mathrm{DMSO}-\mathrm{d}_{6}, 293 \mathrm{~K}\right): \delta 0.53(\mathrm{~d}, J 6.8 \mathrm{~Hz}, 3 \mathrm{H}), 0.70$ $0.77(\mathrm{~m}, 1 \mathrm{H}), 0.79(\mathrm{~d}, J 6.8 \mathrm{~Hz}, 3 \mathrm{H}), 0.83(\mathrm{~d}, J 6.8 \mathrm{~Hz}, 3 \mathrm{H}), 0.83-0.95$ $(\mathrm{m}, 1 \mathrm{H}), 1.21-1.35(\mathrm{~m}, 3 \mathrm{H}), 1.38-1.51(\mathrm{~m}, 1 \mathrm{H}), 1.41(\mathrm{~s}, 18 \mathrm{H}), 1.54-$ $1.58(\mathrm{~m}, 2 \mathrm{H}), 1.84-2.07(\mathrm{~m}, 4 \mathrm{H}), 2.31$ (ddd, J 13.0, 8.2, $6.2 \mathrm{~Hz}, 1 \mathrm{H})$, 2.77-2.85 (m, 2H), 3.54-3.60 (m, 1H), $3.62(\mathrm{dd}, J 11.2,6.6 \mathrm{~Hz}, 1 \mathrm{H})$, $4.22(\mathrm{td}, J) 10.8,4.3 \mathrm{~Hz}, 1 \mathrm{H}), 4.27-4.34(\mathrm{~m}, 1 \mathrm{H}), 4.45(\mathrm{~d}, J 8.5 \mathrm{~Hz}, 1 \mathrm{H})$,

7.26-7.32 (m, 4H), 7.39-7.48 (m, 6H).

${ }^{13} \mathrm{C}$ NMR (100 MHz, DMSO-d, $\left.293 \mathrm{~K}\right): \delta$ 16.23, 20.49, 20.71, 21.74, 22.87, 25.51, 27.55 (3C), 27.71 (3C), 30.54, 33.51, 34.15, 40.47, 46.25, 46.55, 48.14, 59.38, 59.72, 61.56, 65.91, 73.89, $80.11,80.60,127.02$ (2C), 127.59, 127.92, 127.96 (2C), 128.03 (2C), 128.35 (2C), 138.17, 139.14, $167.65,169.95,171.66,172.18$.

HRMS (EI) calculated for $\mathrm{C}_{42} \mathrm{H}_{58} \mathrm{~N}_{2} \mathrm{O}_{7} 702.4239$, found 702.4236 .

4-((1S,2R,5S)-2-isopropyl-5-methylcyclohexyl) 2-methyl $\quad(2 R, 4 R, 5 S)-1-((2 R, 3 S, 5 S)-5-$ (methoxycarbonyl)-2-phenylpyrrolidine-3-carbonyl)-5-phenylpyrrolidine-2,4-dicarboxylate D-2a

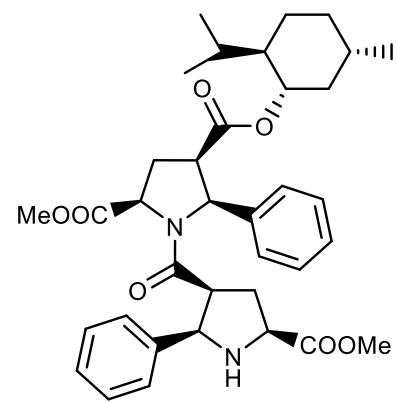
Yield $80 \%, 12.000 \mathrm{~g}$, colorless crystals, m.p. $96-98^{\circ} \mathrm{C},[\alpha]_{D^{21}}+40.4^{\circ}(\mathrm{c}$ $0.955, \mathrm{MeOH})$.

${ }^{1} \mathrm{H}$ NMR $\left(400 \mathrm{MHz}, \mathrm{DMSO}-\mathrm{d}_{6}, 293 \mathrm{~K}\right): \delta 0.51(\mathrm{~d}, J 6.8 \mathrm{~Hz}, 3 \mathrm{H}), 0.70-0.77$ (m, $1 \mathrm{H}), 0.80(\mathrm{~d}, J 6.8 \mathrm{~Hz}, 3 \mathrm{H}), 0.82(\mathrm{~d}, J 6.8 \mathrm{~Hz}, 3 \mathrm{H}), 0.85-0.95(\mathrm{~m}, 1 \mathrm{H})$, 1.21-1.29 (m, 2H), 1.31-1.36 $(\mathrm{m}, 1 \mathrm{H}), 1.39-1.48(\mathrm{~m}, 1 \mathrm{H}), 1.54-1.60(\mathrm{~m}$, $2 \mathrm{H}), 1.85-1.97(\mathrm{~m}, 2 \mathrm{H}), 1.99-2.08(\mathrm{~m}, 2 \mathrm{H}), 2.27$ (ddd, J 13.0, 8.2, $6.0 \mathrm{~Hz}$, $1 \mathrm{H}), 2.88-2.95(\mathrm{~m}, 2 \mathrm{H}), 3.65(\mathrm{~s}, 3 \mathrm{H}), 3.66(\mathrm{~s}, 3 \mathrm{H}), 3.66-3.70(\mathrm{~m}, 2 \mathrm{H})$, $4.23(\mathrm{dd}, J 10.8,4.2 \mathrm{~Hz}, 1 \mathrm{H}), 4.37-4.40(\mathrm{~m}, 1 \mathrm{H}), 4.61(\mathrm{~d}, J 8.4 \mathrm{~Hz}, 1 \mathrm{H})$,

7.27-7.32 (m, 4H), 7.38-7.44 (m, 4H), 7.48-7.51 (m, 2H).

${ }^{13} \mathrm{C}$ NMR $\left(100 \mathrm{MHz}, \mathrm{DMSO}-\mathrm{d}_{6}, 293 \mathrm{~K}\right): \delta 16.17,20.52,21.74,22.80,25.43,27.73,30.55,33.15$, $33.50,40.08,46.19,46.46,48.20,51.66,51.87,58.21,58.88,61.65,65.41,73.91,127.29$ (2C), $127.62,128.00(3 \mathrm{C}), 128.08(2 \mathrm{C}), 128.23$ (2C), 137.97, 139.23, 167.58, 171.65 (2C), 173.20.

Anal. Calcd for $\mathrm{C}_{36} \mathrm{H}_{46} \mathrm{~N}_{2} \mathrm{O}_{7}$ : C 69.88, H 7.49, N 4.53. Found: C 69.53, H 7.49, N 4.36.

4-((1R,2S,5R)-2-isopropyl-5-methylcyclohexyl) 2-methyl $\quad(2 S, 4 S, 5 R)-5$-(3-chlorophenyl)-1((2S,3R,5R)-5-(methoxycarbonyl)-2-(4-methoxyphenyl)pyrrolidine-3-carbonyl)pyrrolidine-2,4dicarboxylate $\mathbf{L - 2 d}$ 


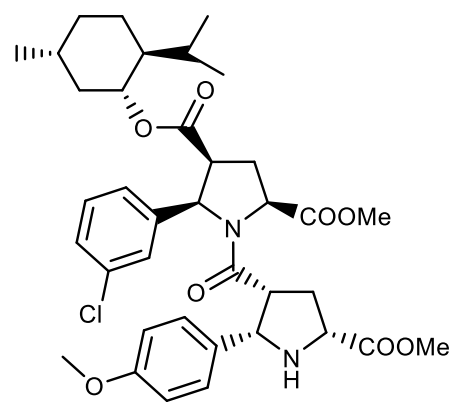

Yield $60 \%, 0.890 \mathrm{~g}$, colorless crystals, m.p. $97-99^{\circ} \mathrm{C},[\alpha]_{D^{21}}+14.1^{\circ}(\mathrm{c}$ 1.13, $\left.\mathrm{CHCl}_{3}\right)$.

${ }^{1} \mathrm{H}$ NMR (400 MHz, DMSO-d $\left.6,293 \mathrm{~K}\right): \delta 0.53(\mathrm{~d}, J 6.7 \mathrm{~Hz}, 3 \mathrm{H}), 0.75-$ $0.81(\mathrm{~m}, 1 \mathrm{H}), 0.82(\mathrm{~s}, 3 \mathrm{H}), 0.84(\mathrm{~s}, 3 \mathrm{H}), 0.86-0.98(\mathrm{~m}, 2 \mathrm{H}), 1.26-1.32$ $(\mathrm{m}, 2 \mathrm{H}), 1.39-1.59(\mathrm{~m}, 4 \mathrm{H}), 1.87-1.94(\mathrm{~m}, 2 \mathrm{H}), 1.99-2.04(\mathrm{~m}, 1 \mathrm{H})$, 2.04-2.14 $(\mathrm{m}, 1 \mathrm{H}), 2.35-2.42(\mathrm{~m}, 1 \mathrm{H}), 2.84-2.89(\mathrm{~m}, 1 \mathrm{H}), 3.65(\mathrm{~s}$, $3 \mathrm{H}), 3.67(\mathrm{~s}, 3 \mathrm{H}), 3.69-3.76(\mathrm{~m}, 2 \mathrm{H}), 3.75(\mathrm{~s}, 3 \mathrm{H}), 4.28(\mathrm{td}, J 10.8$, $4.5 \mathrm{~Hz}, 1 \mathrm{H}), 4.37(\mathrm{~d}, J 7.4 \mathrm{~Hz}, 1 \mathrm{H}), 4.66(\mathrm{~d}, J 8.6 \mathrm{~Hz}, 1 \mathrm{H}), 6.92-6.97$ $(\mathrm{m}, 2 \mathrm{H}), 7.31-7.35(\mathrm{~m}, 4 \mathrm{H}), 7.53-7.58(\mathrm{~m}, 2 \mathrm{H})$.

${ }^{13} \mathrm{C}$ NMR (100 MHz, DMSO-d 6 , $\left.293 \mathrm{~K}\right): \delta 16.01,20.58,21.78,22.69,25.42,27.80,30.60,33.09$, $33.53,40.13,46.18,46.41,48.14,51.64,51.93,55.10,58.30,58.83,61.01,64.77,74.11,113.59$ (2C), 116.16, 126.45, 127.88, 128.12, 128.35 (2C), 130.08, 130.90, 132.86, 140.52, 158.79, $167.56,171.83,173.21$.

HRMS (ESI) calculated for $\mathrm{C}_{37} \mathrm{H}_{48} \mathrm{ClN}_{2} \mathrm{O}_{8}[\mathrm{M}+\mathrm{H}]^{+} 683.3094$, found 683.3120 .

4-((1S,2R,5S)-2-isopropyl-5-methylcyclohexyl) 2-methyl $\quad(2 \mathrm{R}, 4 \mathrm{R}, 5 \mathrm{~S})$-5-(3-chlorophenyl)-1$((2 R, 3 S, 5 S)-5$-(methoxycarbonyl)-2-(4-methoxyphenyl)pyrrolidine-3-carbonyl)pyrrolidine-2,4dicarboxylate $\mathbf{D}-\mathbf{2 d}$

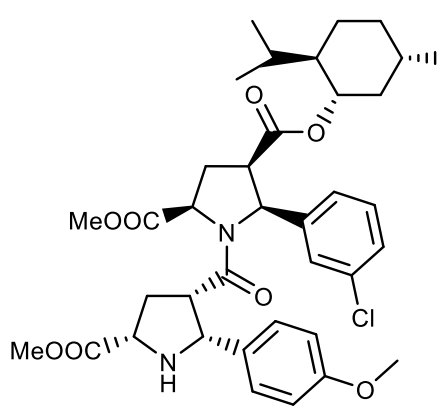

Yield $78 \%, 0.960 \mathrm{~g}$, colorless crystals, m.p. $97-99^{\circ} \mathrm{C},[\alpha]_{D^{21}}-17.1^{\circ}(\mathrm{c}$ $\left.1.13, \mathrm{CHCl}_{3}\right)$.

${ }^{1} \mathrm{H}$ NMR $\left(400 \mathrm{MHz}, \mathrm{DMSO}-\mathrm{d}_{6}, 293 \mathrm{~K}\right): \delta 0.53(\mathrm{~d}, J 6.7 \mathrm{~Hz}, 3 \mathrm{H}), 0.75-$ $0.81(\mathrm{~m}, 1 \mathrm{H}), 0.82(\mathrm{~s}, 3 \mathrm{H}), 0.84(\mathrm{~s}, 3 \mathrm{H}), 0.86-0.98(\mathrm{~m}, 2 \mathrm{H}), 1.26-1.32$ $(\mathrm{m}, 2 \mathrm{H}), 1.39-1.59(\mathrm{~m}, 4 \mathrm{H}), 1.87-1.94(\mathrm{~m}, 2 \mathrm{H}), 1.99-2.04(\mathrm{~m}, 1 \mathrm{H})$, 2.04-2.14 $(\mathrm{m}, 1 \mathrm{H}), 2.35-2.42(\mathrm{~m}, 1 \mathrm{H}), 2.84-2.89(\mathrm{~m}, 1 \mathrm{H}), 3.65(\mathrm{~s}$, $3 \mathrm{H}), 3.67(\mathrm{~s}, 3 \mathrm{H}), 3.69-3.76(\mathrm{~m}, 2 \mathrm{H}), 3.75(\mathrm{~s}, 3 \mathrm{H}), 4.28(\mathrm{td}, J \mathrm{~J} 10.8,4.5$ $\mathrm{Hz}, 1 \mathrm{H}), 4.37(\mathrm{~d}, J 7.4 \mathrm{~Hz}, 1 \mathrm{H}), 4.66(\mathrm{~d}, J 8.6 \mathrm{~Hz}, 1 \mathrm{H}), 6.92-6.97(\mathrm{~m}$, $2 \mathrm{H})$, 7.31-7.35 (m, 4H), 7.53-7.58 (m, $2 \mathrm{H})$.

${ }^{13} \mathrm{C}$ NMR $(100 \mathrm{MHz}$, DMSO-d $6,293 \mathrm{~K}): \delta 16.01,20.58,21.78,22.69,25.42,27.80,30.60,33.09$, $33.53,40.13,46.18,46.41,48.14,51.64,51.93,55.10,58.30,58.83,61.01,64.77,74.11,113.59$ (2C), 116.16, 126.45, 127.88, 128.12, 128.35 (2C), 130.08, 130.90, 132.86, 140.52, 158.79, $167.56,171.83,173.21$.

HRMS (ESI) calculated for $\mathrm{C}_{37} \mathrm{H}_{48} \mathrm{ClN}_{2} \mathrm{O}_{8}[\mathrm{M}+\mathrm{H}]^{+} 683.3094$, found 683.3120 .

4-((1R,2S,5R)-2-isopropyl-5-methylcyclohexyl) 2-methyl $\quad(2 S, 4 S, 5 R)-1-((2 S, 3 R, 5 R)-1$-acryloyl-5-

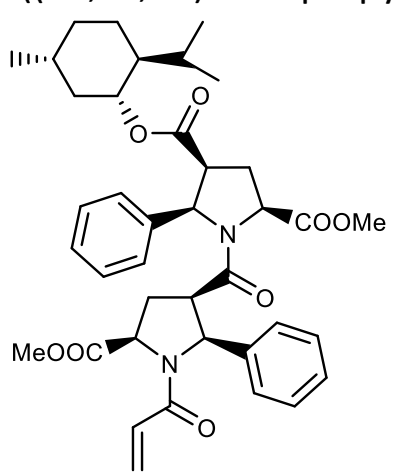
(methoxycarbonyl)-2-phenylpyrrolidine-3-carbonyl)-5phenylpyrrolidine-2,4-dicarboxylate L-2a_A

Yield $94 \%, 2.470 \mathrm{~g}$, colorless crystals, m.p. $117-119^{\circ} \mathrm{C},[\alpha]_{D}^{21}+52.8^{\circ}(\mathrm{c}$ $0.64, \mathrm{MeOH})$.

${ }^{1} \mathrm{H}$ NMR (400 MHz, DMSO-d $\left.6,293 \mathrm{~K}\right): \delta 0.50(\mathrm{~d}, J 6.8 \mathrm{~Hz}, 3 \mathrm{H}), 0.72-$ $0.81(\mathrm{~m}, 1 \mathrm{H}), 0.76(\mathrm{~d}, J 6.8 \mathrm{~Hz}, 3 \mathrm{H}), 0.83(\mathrm{~d}, J 6.8 \mathrm{~Hz}, 3 \mathrm{H}), 0.84-0.93(\mathrm{~m}$, $2 \mathrm{H}), 1.25-1.31(\mathrm{~m}, 2 \mathrm{H}), 1.40-1.50(\mathrm{~m}, 2 \mathrm{H}), 1.53-1.62(\mathrm{~m}, 2 \mathrm{H}), 1.76-1.82$ $(\mathrm{m}, 1 \mathrm{H}), 2.14-2.26(\mathrm{~m}, 2 \mathrm{H}), 2.30-2.37(\mathrm{~m}, 1 \mathrm{H}), 3.11$ (ddd, J 12.0, 8.4, $6.2 \mathrm{~Hz}, 1 \mathrm{H}), 3.60(\mathrm{~s}, 3 \mathrm{H}), 3.67(\mathrm{~s}, 3 \mathrm{H}), 3.71-3.83(\mathrm{~m}, 2 \mathrm{H}), 4.10(\mathrm{dd}, J$ $11.3,6.8 \mathrm{~Hz}, 1 \mathrm{H}$ ), 4.38 (td, J 10.8, $4.2 \mathrm{~Hz}, 1 \mathrm{H}$ ), 5.55 (dd, J 10.0, $2.4 \mathrm{~Hz}$, $1 \mathrm{H}), 5.84(\mathrm{~d}, J 8.6 \mathrm{~Hz}, 1 \mathrm{H}), 5.96(\mathrm{~d}, J 8.4 \mathrm{~Hz}, 1 \mathrm{H}), 6.08(\mathrm{dd}, J$ 16.8, $2.4 \mathrm{~Hz}, 1 \mathrm{H}), 6.20$ (dd, J 16.8, $10.0 \mathrm{~Hz}, 1 \mathrm{H}), 7.23-7.35(\mathrm{~m}, 6 \mathrm{H}), 7.50(\mathrm{~d}, J 7.4 \mathrm{~Hz}, 2 \mathrm{H}), 7.72-7.75(\mathrm{~m}, 2 \mathrm{H})$. 
${ }^{13} \mathrm{C}$ NMR (100 MHz, DMSO-d $\left.6,293 \mathrm{~K}\right): \delta$ 15.68, 20.43, 21.58, 22.33, 24.87, 28.31, 28.76, 30.43, 33.32 , 40.06, 45.85, 46.98, 48.63, 51.59, 51.65, 58.32, 58.75, 60.23, 60.89, 73.71, 126.61 (2C), 127.33, 127.70 (2C), 127.81 (2C), 127.89 (2C), 127.99 (2C), 128.55, 138.55, 139.65, 163.84, $166.77,167.88,171.51,171.73$.

Anal. Calcd for $\mathrm{C}_{39} \mathrm{H}_{48} \mathrm{~N}_{2} \mathrm{O}_{8}$ : C 69.62, H 7.19, N 4.16. Found: C 69.42, H 7.05, N 3.96.

4-((1R,2S,5R)-2-isopropyl-5-methylcyclohexyl) 2-methyl $(2 S, 4 S, 5 R)-1-((2 S, 3 R, 5 R)$-1-acryloyl-2-(4-

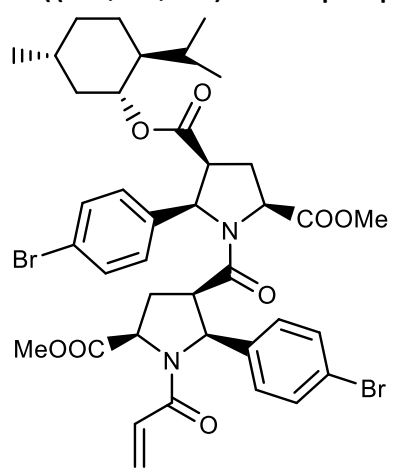
bromophenyl)-5-(methoxycarbonyl)pyrrolidine-3-carbonyl)-5-(4bromophenyl)pyrrolidine-2,4-dicarboxylate L-2b_A Yield $80 \%, 4.560 \mathrm{~g}$, colorless crystals, m.p. $135-137^{\circ} \mathrm{C},[\alpha]_{D}{ }^{20}+26.0^{\circ}(\mathrm{c}$ $\left.1.13, \mathrm{CHCl}_{3}\right)$.

${ }^{1} \mathrm{H} \mathrm{NMR}\left(400 \mathrm{MHz}\right.$, DMSO- $\left.\mathrm{d}_{6}, 293 \mathrm{~K}\right): \delta 0.49(\mathrm{~d}, J 6.1 \mathrm{~Hz}, 3 \mathrm{H}), 0.72-0.81$ $(\mathrm{m}, 1 \mathrm{H}), 0.75(\mathrm{~d}, J 6.5 \mathrm{~Hz}, 3 \mathrm{H}), 0.83(\mathrm{~d}, J 6.5 \mathrm{~Hz}, 3 \mathrm{H}), 0.85-0.95(\mathrm{~m}, 2 \mathrm{H})$, $1.19-1.36(\mathrm{~m}, 3 \mathrm{H}), 1.43-1.48(\mathrm{~m}, 1 \mathrm{H}), 1.53-1.60(\mathrm{~m}, 2 \mathrm{H}), 1.81-1.88(\mathrm{~m}$, $1 \mathrm{H}), 2.07-2.23(\mathrm{~m}, 2 \mathrm{H}), 2.34-2.40(\mathrm{~m}, 1 \mathrm{H}), 3.07-3.14(\mathrm{~m}, 1 \mathrm{H}), 3.61(\mathrm{~s}$, $3 \mathrm{H}), 3.66(\mathrm{~s}, 3 \mathrm{H}), 3.76-3.80(\mathrm{~m}, 1 \mathrm{H}), 3.84-3.91(\mathrm{~m}, 1 \mathrm{H}), 4.13(\mathrm{dd}, J$ 11.4, $6.8 \mathrm{~Hz}, 1 \mathrm{H}), 4.36-4.43(\mathrm{~m}, 1 \mathrm{H}), 5.59(\mathrm{dd}, J 9.8,2.6 \mathrm{~Hz}, 1 \mathrm{H}), 5.88(\mathrm{~d}, J 8.7$ Hz, 1H), 5.98 (d, J 8.5 Hz, 1H), 6.09 (dd, J 16.8, 2.6 Hz, 1H), 6.19 (dd, J 16.8, 9.8 Hz, 1H), 7.15-7$74(\mathrm{~m}, 8 \mathrm{H})$.

${ }^{13} \mathrm{C}$ NMR (100 MHz, DMSO-d $\left.6,293 \mathrm{~K}\right): \delta$ 15.54, 20.39, 21.58, 22.20, 24.88, 28.30, 28.77, 30.39, 33.26, 40.06, 45.75, 46.91, 48.69, 51.72 (2C), 58.27, 58.84, 59.47, 60.23, 73.82, 120.65, 121.45, $128.16,128.39,128.81$ (2C), 129.85 (2C), 130.95 (2C), 137.92, 139.27, 163.74, 166.73, 167.78, $170.56,171.94$.

HRMS (EI) calculated for $\mathrm{C}_{39} \mathrm{H}_{46}{ }^{79} \mathrm{Br}_{2} \mathrm{~N}_{2} \mathrm{O}_{8} 828.1620$, found 828.1622 .

2-(tert-butyl) 4-((1R,2S,5R)-2-isopropyl-5-methylcyclohexyl) $(2 S, 4 S, 5 R)-1-((2 S, 3 R, 5 R)$-1-acryloyl5-(tert-butoxycarbonyl)-2-phenylpyrrolidine-3-carbonyl)-5-phenylpyrrolidine-2,4-dicarboxylate L-2c_A

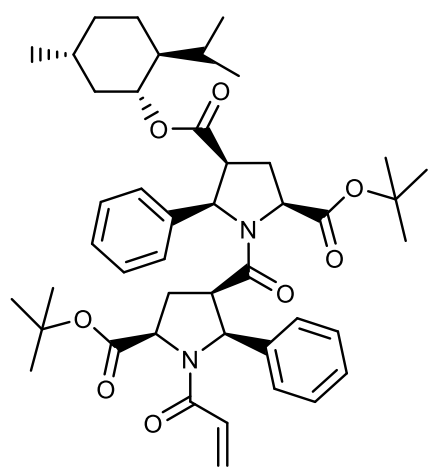

Yield $83 \%, 7.800 \mathrm{~g}$, colorless crystals, m.p. $114-116^{\circ} \mathrm{C},[\alpha]_{D}{ }^{21}+31.9^{\circ}$ (c $0.69, \mathrm{MeOH}$ ).

${ }^{1} \mathrm{H}$ NMR (400 MHz, DMSO-d $\left.6,293 \mathrm{~K}\right): \delta$ 0.51-0.56 (m, 3H), 0.76-0.94 $(\mathrm{m}, 9 \mathrm{H}), 1.15-1.58(\mathrm{~m}, 23 \mathrm{H}), 1.71-1.77(\mathrm{~m}, 1 \mathrm{H}), 2.04-2.23(\mathrm{~m}, 3 \mathrm{H})$, 2.25-2.34 (m, $1 \mathrm{H}), 2.98-3.07(\mathrm{~m}, 1 \mathrm{H}), 3.58-3.68(\mathrm{~m}, 1 \mathrm{H}), 3.70-3.84$ (m, $1 \mathrm{H}), 3.91(\mathrm{dd}, J 11.4,6.8 \mathrm{~Hz}, 1 \mathrm{H}), 4.34-4.43(\mathrm{~m}, 1 \mathrm{H}), 5.53(\mathrm{dd}, J$ 9.7, $2.8 \mathrm{~Hz}, 1 \mathrm{H}), 5.81(\mathrm{~d}, J 8.0 \mathrm{~Hz}, 1 \mathrm{H}), 5.92(\mathrm{~d}, J 8.4 \mathrm{~Hz}, 1 \mathrm{H}), 6.04-$ $6.21(\mathrm{~m}, 2 \mathrm{H}), 7.23-7.39(\mathrm{~m}, 5 \mathrm{H}), 7.45-7.61(\mathrm{~m}, 3 \mathrm{H}), 7.71-7.73(\mathrm{~m}$, $2 \mathrm{H})$.

${ }^{13} \mathrm{C}$ NMR (100 MHz, DMSO-d $\left.6,293 \mathrm{~K}\right): \delta$ 15.95, 20.61, 21.80, 22.60, $25.17,27.56$ (8C), 30.63, 33.52, 40.24, 46.11 (2C), 47.10, 59.54, 60.06, 60.34, 61.23, 73.88, $80.11,80.65,126.88$ (2C), 127.94 (4C), 128.01 (4C), 128.13 (2C), 138.87, 139.98, 163.96, 166.84, $168.22,169.95,170.22$.

HRMS (EI) calculated for $\mathrm{C}_{45} \mathrm{H}_{60} \mathrm{~N}_{2} \mathrm{O}_{8} 756.4344$, found 756.4343 . 
4-((1S,2R,5S)-2-isopropyl-5-methylcyclohexyl) 2-methyl (2R,4R,5S)-1-((2R,3S,5S)-1-acryloyl-5-

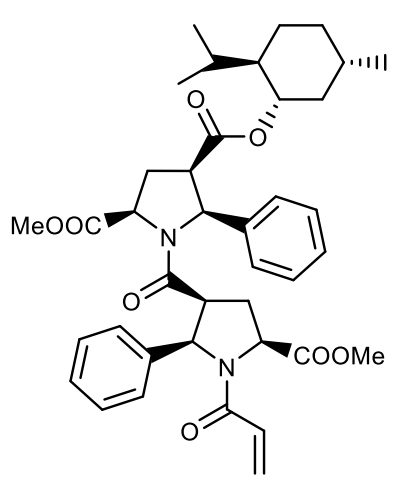

(methoxycarbonyl)-2-phenylpyrrolidine-3-carbonyl)-5-

phenylpyrrolidine-2,4-dicarboxylate D-2a_A

Yield $90 \%, 10.350$ g, yellowish crystals, m.p. $117-119{ }^{\circ} \mathrm{C},[\alpha]_{D}{ }^{21}-52.0^{\circ}$ (c $1.01, \mathrm{MeOH})$.

${ }^{1} \mathrm{H}$ NMR $\left(400 \mathrm{MHz}, \mathrm{DMSO}-\mathrm{d}_{6}, 293 \mathrm{~K}\right): \delta 0.50(\mathrm{~d}, J 6.8 \mathrm{~Hz}, 3 \mathrm{H}), 0.72-0.81$ $(\mathrm{m}, 1 \mathrm{H}), 0.76(\mathrm{~d}, J 6.8 \mathrm{~Hz}, 3 \mathrm{H}), 0.83(\mathrm{~d}, J 6.8 \mathrm{~Hz}, 3 \mathrm{H}), 0.84-0.93(\mathrm{~m}, 2 \mathrm{H})$, $1.25-1.31(\mathrm{~m}, 2 \mathrm{H}), 1.40-1.50(\mathrm{~m}, 2 \mathrm{H}), 1.53-1.62(\mathrm{~m}, 2 \mathrm{H}), 1.76-1.82(\mathrm{~m}$, $1 \mathrm{H}), 2.14-2.26(\mathrm{~m}, 2 \mathrm{H}), 2.30-2.37(\mathrm{~m}, 1 \mathrm{H}), 3.11$ (ddd, J 12.0, 8.4, $6.2 \mathrm{~Hz}$, $1 \mathrm{H}), 3.60(\mathrm{~s}, 3 \mathrm{H}), 3.67(\mathrm{~s}, 3 \mathrm{H}), 3.71-3.83(\mathrm{~m}, 2 \mathrm{H}), 4.10(\mathrm{dd}, J 11.3,6.8$ $\mathrm{Hz}, 1 \mathrm{H}), 4.38(\mathrm{td}, J 10.8,4.2 \mathrm{~Hz}, 1 \mathrm{H}), 5.55(\mathrm{dd}, J 10.0,2.4 \mathrm{~Hz}, 1 \mathrm{H}), 5.84$ (d, J $8.6 \mathrm{~Hz}, 1 \mathrm{H}$ ), 5.96 (d, J $8.4 \mathrm{~Hz}, 1 \mathrm{H}), 6.08$ (dd, J 16.8, $2.4 \mathrm{~Hz}, 1 \mathrm{H}$ ), 6.20 (dd, J 16.8, $10.0 \mathrm{~Hz}, 1 \mathrm{H}$ ), 7.23-7.35 (m, 6H), $7.50(\mathrm{~d}, J 7.4 \mathrm{~Hz}, 2 \mathrm{H}), 7.72-7.75(\mathrm{~m}, 2 \mathrm{H})$.

${ }^{13} \mathrm{C}$ NMR $(100 \mathrm{MHz}$, DMSO-d, $293 \mathrm{~K}): \delta 15.68,20.43,21.58,22.33,24.87,28.31,28.76,30.43$, $33.32,40.06,45.85,46.98,48.63,51.59,51.65,58.32,58.75,60.23,60.89,73.71,126.61$ (2C), $127.33,127.70(2 \mathrm{C}), 127.81$ (2C), 127.89 (2C), 127.99 (2C), 128.55, 138.55, 139.65, 163.84, $166.77,167.88,171.51,171.73$.

Anal. Calcd for $\mathrm{C}_{39} \mathrm{H}_{48} \mathrm{~N}_{2} \mathrm{O}_{8}$ : C 69.62, H 7.19, N 4.16. Found: C 69.42, H 7.05, N 3.96.

HRMS (EI) calculated for $\mathrm{C}_{39} \mathrm{H}_{49} \mathrm{~N}_{2} \mathrm{O}_{8}[\mathrm{M}+\mathrm{H}]^{+} 673.3483$, found 673.3483 .

4-((1R,2S,5R)-2-isopropyl-5-methylcyclohexyl) 2-methyl $\quad(2 S, 4 S, 5 R)-1-((2 S, 3 R, 5 R)$-1-acryloyl-5-

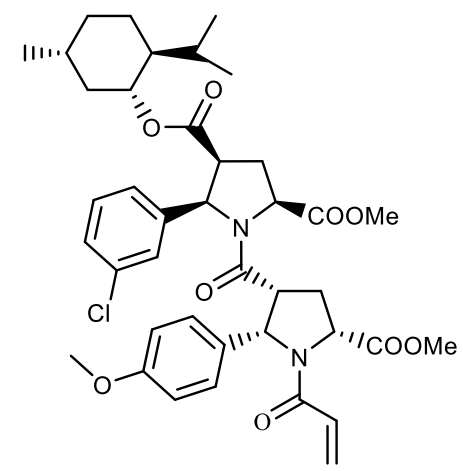
(methoxycarbonyl)-2-(4-methoxyphenyl)pyrrolidine-3-carbonyl)-5(3-chlorophenyl)pyrrolidine-2,4-dicarboxylate L-2d_A

Yield $93 \%, 0.715 \mathrm{~g}$, colorless crystals, m.p. $148-150{ }^{\circ} \mathrm{C},[\alpha]_{D}{ }^{21}$ $-57.9^{\circ}$ (c 1.13, MeOH).

${ }^{1} \mathrm{H}$ NMR $\left(400 \mathrm{MHz}, \mathrm{DMSO}-\mathrm{d}_{6}, 293 \mathrm{~K}\right): \delta 0.53(\mathrm{~d}, \mathrm{~J} 7.0 \mathrm{~Hz}, 3 \mathrm{H}), 0.76-$ $0.81(\mathrm{~m}, 1 \mathrm{H}), 0.79(\mathrm{~d}, J 7.0 \mathrm{~Hz}, 3 \mathrm{H}), 0.86(\mathrm{~d}, J 7.0 \mathrm{~Hz}, 3 \mathrm{H}), 0.86-0.98$ $(\mathrm{m}, 2 \mathrm{H}), 1.27-1.35(\mathrm{~m}, 2 \mathrm{H}), 1.45-1.62(\mathrm{~m}, 4 \mathrm{H}), 1.80-1.86(\mathrm{~m}, 1 \mathrm{H})$, 2.11-2.23 (m, 2H), 2.32-2.38 (m, $1 \mathrm{H}), 3.02-3.08(\mathrm{~m}, 1 \mathrm{H}), 3.61(\mathrm{~s}$, $3 \mathrm{H}), 3.66(\mathrm{~s}, 3 \mathrm{H}), 3.71-3.84(\mathrm{~m}, 2 \mathrm{H}), 3.74(\mathrm{~s}, 3 \mathrm{H}), 4.15(\mathrm{dd}, J 11.3$, $6.9 \mathrm{~Hz}, 1 \mathrm{H}), 4.41(\mathrm{td}, J 10.8,4.2 \mathrm{~Hz}, 1 \mathrm{H}), 5.56(\mathrm{dd}, J 10.1,2.2 \mathrm{~Hz}$, 1H), 5.82 (d, J $8.6 \mathrm{~Hz}, 1 \mathrm{H}), 5.89(\mathrm{~d}, J 8.4 \mathrm{~Hz}, 1 \mathrm{H}), 6.08$ (dd, J 16.8, 2.2 Hz, 1H), 6.21 (dd, J 16.8, $10.1 \mathrm{~Hz}, 1 \mathrm{H}), 6.89$ (d, J 8.6 Hz, 2H), 7.29-7.39 (m, 2H), 7.42 (d, J 8.6 Hz, 2H), 7.75-7.78 (m, 2H).

${ }^{13} \mathrm{C}$ NMR $(100 \mathrm{MHz}$, DMSO-d $6,293 \mathrm{~K}): \delta$ 16.07, 20.81, 22.02, 22.77, 25.45, 28.66, 29.14, 30.88, $33.76,40.50,46.28,47.40,48.98,52.02,52.09,55.07,58.70,59.20,60.20,60.60,74.34,113.80$ (2C), 126.56, 127.99, 128.08, 128.16 (2C), 128.35, 129.02, 130.25, 131.94, 133.12, 141.41, $158.68,164.23,167.41,168.19,172.07,172.24$.

Anal. Calcd for $\mathrm{C}_{40} \mathrm{H}_{49} \mathrm{ClN}_{2} \mathrm{O}_{9}$ : C 65.16, H 6.70, N 3.80. Found: C 64.91, H 6.70, N 3.76.

4-((1S,2R,5S)-2-isopropyl-5-methylcyclohexyl) 2-methyl $\quad(2 R, 4 R, 5 S)-1-((2 R, 3 S, 5 S)$-1-acryloyl-5(methoxycarbonyl)-2-(4-methoxyphenyl)pyrrolidine-3-carbonyl)-

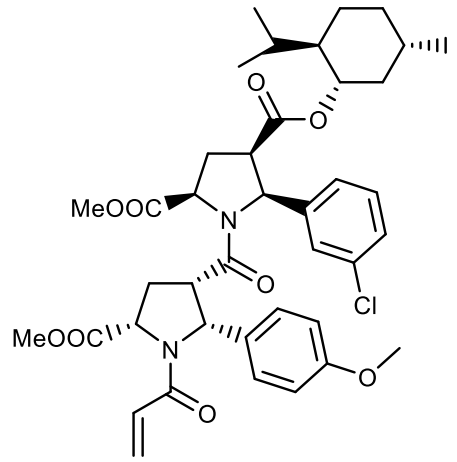
5-(3-chlorophenyl)pyrrolidine-2,4-dicarboxylate D-2d_A Yield $81 \%, 0.799 \mathrm{~g}$, colorless crystals, m.p. $148-150{ }^{\circ} \mathrm{C},[\alpha]_{D}{ }^{21}$ $+44.6^{\circ}$ (c $\left.0.34, \mathrm{MeOH}\right)$.

${ }^{1} \mathrm{H} \mathrm{NMR}\left(400 \mathrm{MHz}, \mathrm{DMSO}-\mathrm{d}_{6}, 293 \mathrm{~K}\right): \delta 0.53(\mathrm{~d}, J 7.0 \mathrm{~Hz}, 3 \mathrm{H}), 0.76-$ $0.81(\mathrm{~m}, 1 \mathrm{H}), 0.79(\mathrm{~d}, J 7.0 \mathrm{~Hz}, 3 \mathrm{H}), 0.86(\mathrm{~d}, J 7.0 \mathrm{~Hz}, 3 \mathrm{H}), 0.86-$ $0.98(\mathrm{~m}, 2 \mathrm{H}), 1.27-1.35(\mathrm{~m}, 2 \mathrm{H}), 1.45-1.62(\mathrm{~m}, 4 \mathrm{H}), 1.80-1.86(\mathrm{~m}$, 
$1 \mathrm{H}), 2.11-2.23(\mathrm{~m}, 2 \mathrm{H}), 2.32-2.38(\mathrm{~m}, 1 \mathrm{H}), 3.02-3.08(\mathrm{~m}, 1 \mathrm{H}), 3.61(\mathrm{~s}, 3 \mathrm{H}), 3.66(\mathrm{~s}, 3 \mathrm{H}), 3.71-3.84$ $(\mathrm{m}, 2 \mathrm{H}), 3.74(\mathrm{~s}, 3 \mathrm{H}), 4.15(\mathrm{dd}, J 11.3,6.9 \mathrm{~Hz}, 1 \mathrm{H}), 4.41(\mathrm{td}, J 10.8,4.2 \mathrm{~Hz}, 1 \mathrm{H}), 5.56$ (dd, J 10.1, $2.2 \mathrm{~Hz}, 1 \mathrm{H}), 5.82(\mathrm{~d}, J 8.6 \mathrm{~Hz}, 1 \mathrm{H}), 5.89(\mathrm{~d}, J 8.4 \mathrm{~Hz}, 1 \mathrm{H}), 6.08(\mathrm{dd}, J$ 16.8, $2.2 \mathrm{~Hz}, 1 \mathrm{H}), 6.21$ (dd, J $16.8,10.1 \mathrm{~Hz}, 1 \mathrm{H}), 6.89(\mathrm{~d}, J 8.6 \mathrm{~Hz}, 2 \mathrm{H}), 7.29-7.39(\mathrm{~m}, 2 \mathrm{H}), 7.42(\mathrm{~d}, J 8.6 \mathrm{~Hz}, 2 \mathrm{H}), 7.75-7.78(\mathrm{~m}$, $2 \mathrm{H})$.

${ }^{13} \mathrm{C}$ NMR (100 MHz, DMSO-d $\left.6,293 \mathrm{~K}\right): \delta$ 16.07, 20.81, 22.02, 22.77, 25.45, 28.66, 29.14, 30.88, $33.76,40.50,46.28,47.40,48.98,52.02,52.09,55.07,58.70,59.20,60.20,60.60,74.34,113.80$ (2C), 126.56, 127.99, 128.08, 128.16 (2C), 128.35, 129.02, 130.25, 131.94, 133.12, 141.41, $158.68,164.23,167.41,168.19,172.07,172.24$.

4-((1R,2S,5R)-2-isopropyl-5-methylcyclohexyl) $\quad 2$-methyl $\quad(2 S, 4 S, 5 R)-1-((2 S, 3 R, 5 R)-5-$

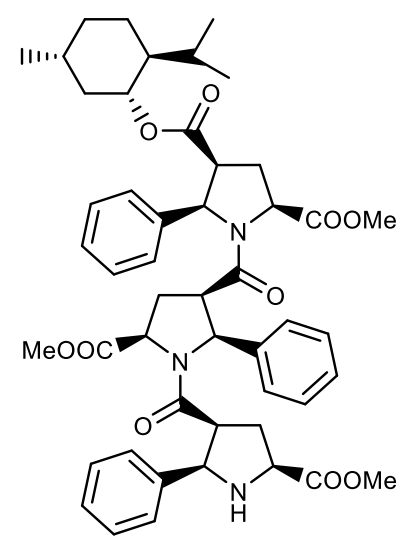
(methoxycarbonyl)-1-((2R,3S,5S)-5-(methoxycarbonyl)-2phenylpyrrolidine-3-carbonyl)-2-phenylpyrrolidine-3-carbonyl)-5phenylpyrrolidine-2,4-dicarboxylate L-3a Yield $89 \%, 1.680 \mathrm{~g}$, colorless crystals, m.p. $144-146{ }^{\circ} \mathrm{C},[\alpha]_{D}{ }^{21}-20.4^{\circ}(\mathrm{c}$ 1.01, $\mathrm{MeOH})$.

${ }^{1} \mathrm{H}$ NMR $(400 \mathrm{MHz}$, DMSO-d $6,293 \mathrm{~K}): \delta 0.52(\mathrm{~d}, J 6.8 \mathrm{~Hz}, 3 \mathrm{H}), 0.71-0.82$ $(\mathrm{m}, 1 \mathrm{H}), 0.77(\mathrm{~d}, J 6.8 \mathrm{~Hz}, 3 \mathrm{H}), 0.85(\mathrm{~d}, J 6.8 \mathrm{~Hz}, 3 \mathrm{H}), 0.85-0.98(\mathrm{~m}, 2 \mathrm{H})$, 1.23-1.34 (m, 2H), 1.41-1.47 (m, 1H), 1.50-1.61 (m, 4H), $1.68(\mathrm{dt}, J$ 12.5, 7.6 Hz, 1H), 1.92 (ddd, J 12.5, 9.3, 7.6 Hz, 1H), 1.98-2.07 (m, $1 \mathrm{H})$, 2.14-2.23 (m, 1H), 2.29-2.37 (m, 1H), 2.82 (ddd, J 11.5, 8.2, $6.6 \mathrm{~Hz}$, $1 \mathrm{H}), 3.00-3.07(\mathrm{~m}, 1 \mathrm{H}), 3.08-3.13(\mathrm{~m}, 2 \mathrm{H}), 3.53(\mathrm{~s}, 3 \mathrm{H}), 3.59(\mathrm{~s}, 3 \mathrm{H})$, $3.64(\mathrm{~s}, 3 \mathrm{H}), 3.64-3.69(\mathrm{~m}, 1 \mathrm{H}), 3.74-3.80(\mathrm{~m}, 2 \mathrm{H}), 4.40(\mathrm{td}, J \mathrm{~J} 10.9,4.3$ $\mathrm{Hz}, 1 \mathrm{H}), 4.66(\mathrm{t}, J 7.6 \mathrm{~Hz}, 1 \mathrm{H}), 5.61(\mathrm{~d}, J 8.6 \mathrm{~Hz}, 1 \mathrm{H}), 5.90(\mathrm{~d}, J 8.6 \mathrm{~Hz}, 1 \mathrm{H}), 7.10-7.19(\mathrm{~m}, 3 \mathrm{H})$, 7.20-7.30 (m, 2H), 7.33-7.43 (m, 6H), $7.60(\mathrm{~d}, J 7.3 \mathrm{~Hz}, 2 \mathrm{H}), 7.72-7.75(\mathrm{~m}, 2 \mathrm{H})$.

${ }^{13} \mathrm{C}$ NMR $\left(100 \mathrm{MHz}, \mathrm{DMSO}-\mathrm{d}_{6}, 293 \mathrm{~K}\right): \delta 15.92,20.64,21.80,22.55,25.11,28.60,29.06,30.65$, $30.91,33.35,33.51,40.25$, 46.11, 46.42, 46.96, 48.98, 51.69, 51.81, 58.23, 58.98, 59.13, 60.47, $61.19,63.21,74.03,126.86$ (3C), 126.97 (2C), 127.49 (2C), 127.53 (2C), 127.64, 128.08 (2C), $128.17,128.40(2 \mathrm{C}), 138.79,139.35,141.04,166.94,168.19,171.27,171.57,171.64,173.32$. Anal. Calcd for $\mathrm{C}_{49} \mathrm{H}_{59} \mathrm{~N}_{3} \mathrm{O}_{10}$ : C 69.24, H 7.00, N 4.94. Found: $\mathrm{C} 68.83, \mathrm{H} 6.79, \mathrm{~N} 4.71$.

4-((1R,2S,5R)-2-isopropyl-5-methylcyclohexyl) 2-methyl $\quad(2 S, 4 S, 5 R)-5$-(4-bromophenyl)-1((2S,3R,5R)-2-(4-bromophenyl)-1-((2R,3S,5S)-2-(4-bromophenyl)-5-(methoxycarbonyl)-

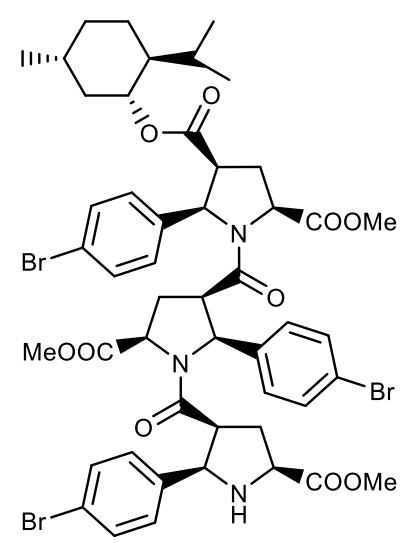
pyrrolidine-3-carbonyl)-5-(methoxycarbonyl)pyrrolidine-3carbonyl)pyrrolidine-2,4-dicarboxylate L-3b Yield $89 \%, 5.100$ g, colorless crystals, m.p. $155-157^{\circ} \mathrm{C},[\alpha]_{D}^{21}-24.4^{\circ}$ (c $\left.1.13, \mathrm{CHCl}_{3}\right)$.

${ }^{1} \mathrm{H}$ NMR $\left(400 \mathrm{MHz}, \mathrm{DMSO}-\mathrm{d}_{6}, 293 \mathrm{~K}\right): \delta 0.51(\mathrm{~d}, J 6.8 \mathrm{~Hz}, 3 \mathrm{H}), 0.71-0.82$ $(\mathrm{m}, 1 \mathrm{H}), 0.77(\mathrm{~d}, J 6.8 \mathrm{~Hz}, 3 \mathrm{H}), 0.85(\mathrm{~d}, J 6.8 \mathrm{~Hz}, 3 \mathrm{H}), 0.85-0.98(\mathrm{~m}, 2 \mathrm{H})$, 1.23-1.43 (m, 4H), 1.48-1.61 (m, 4H), 1.65-1.76 (m, 1H), 1.85-2.02 (m, $2 \mathrm{H}), 2.10-2.22(\mathrm{~m}, 1 \mathrm{H}), 2.33-2.41(\mathrm{~m}, 1 \mathrm{H}), 2.77-2.85(\mathrm{~m}, 1 \mathrm{H}), 3.09(\mathrm{q}, J$ $8.0 \mathrm{~Hz}, 1 \mathrm{H}$ ), 3.19 (dd, J 11.0, $7.0 \mathrm{~Hz}, 1 \mathrm{H}), 3.54(\mathrm{~s}, 3 \mathrm{H}), 3.61(\mathrm{~s}, 3 \mathrm{H}), 3.64$ (s, 3H), 3.64-3.72 (m, 1H), 3.83-3.92 (m, 2H), $4.42(\mathrm{td}, J 10.7,4.3 \mathrm{~Hz}$, $1 \mathrm{H}), 4.68(\mathrm{~d}, J 8.5 \mathrm{~Hz}, 1 \mathrm{H}), 5.75(\mathrm{~d}, J 8.6 \mathrm{~Hz}, 1 \mathrm{H}), 6.06(\mathrm{~d}, J 8.6 \mathrm{~Hz}, 1 \mathrm{H})$, 7.21-7.29 (m, 4H), $7.56(\mathrm{~s}, 4 \mathrm{H})$, 7.61-7.69 (m, 4H).

${ }^{13} \mathrm{C}$ NMR $\left(100 \mathrm{MHz}, \mathrm{CDCl}_{3}, 293 \mathrm{~K}\right): \delta 16.01,20.75,21.94,22.05,22.98,25.76,28.16,29.45$, 31.22 , 33.89, 34.48, 40.73, 46.61, 47.42, 47.50, 50.14, 52.28, 58.81, 59.11, 59.80, 62.04, 62.16, 
$65.47,66.05,75.73,122.06,122.85,123.33,128.52,128.75,128.91,129.40,129.74,130.81$, $131.19,131.29,131.67,131.82,132.18,132.35,136.13,137.41,137.71,167.29,167.57,171.37$, $171.48,171.74,173.02$.

Anal. Calcd for $\mathrm{C}_{49} \mathrm{H}_{56} \mathrm{Br}_{3} \mathrm{~N}_{3} \mathrm{O}_{10}$ : C 54.16, H 5.19, N 3.87. Found: C 53.75, H 5.15, N 3.56. HRMS (ESI) calculated for $\mathrm{C}_{49} \mathrm{H}_{57} \mathrm{Br}_{3} \mathrm{O}_{10} \mathrm{~N}_{3}[\mathrm{M}+\mathrm{H}]^{+} 1084.1589$, found 1084.1603.

2-(tert-butyl) 4-((1R,2S,5R)-2-isopropyl-5-methylcyclohexyl) $\quad(2 S, 4 S, 5 R)-1-((2 S, 3 R, 5 R)-5-($ tertbutoxycarbonyl)-1-((2R,3S,5S)-5-(tert-butoxycarbonyl)-2-phenylpyrrolidine-3-carbonyl)-2phenylpyrrolidine-3-carbonyl)-5-phenylpyrrolidine-2,4-dicarboxylate L-3C

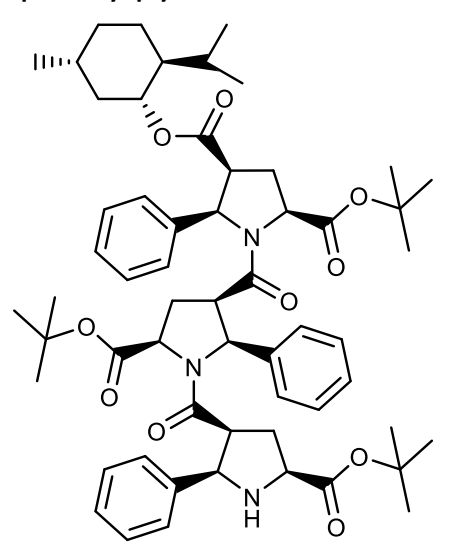

Yield $89 \%, 8.500 \mathrm{~g}$, colorless crystals, m.p. $127-129^{\circ} \mathrm{C},[\alpha]_{D}{ }^{21}-16.6^{\circ}$ (c 1.71, MeOH).

${ }^{1} \mathrm{H}$ NMR $\left(400 \mathrm{MHz}, \mathrm{CDCl}_{3}, 293 \mathrm{~K}\right): \delta$ 0.54-0.59 (m, 3H), 0.77-1.01 (m, $10 \mathrm{H}), 1.19-1.71(\mathrm{~m}, 37 \mathrm{H}), 1.93-1.97(\mathrm{~m}, 1 \mathrm{H}), 2.18-2.42(\mathrm{~m}, 3 \mathrm{H}), 2.69-$ $2.73(\mathrm{~m}, 1 \mathrm{H}), 2.93-2.98(\mathrm{~m}, 1 \mathrm{H}), 3.20-3.27(\mathrm{~m}, 1 \mathrm{H}), 3.63-3.68(\mathrm{~m}, 2 \mathrm{H})$, $4.34(\mathrm{~d}, J 7.2 \mathrm{~Hz}, 1 \mathrm{H}), 4.52$ (td, J 10.8, $4.1 \mathrm{~Hz}, 1 \mathrm{H}), 4.73$ (d, J $8.6 \mathrm{~Hz}$, $1 \mathrm{H}), 5.08(\mathrm{~d}, J 8.4 \mathrm{~Hz}, 1 \mathrm{H}), 7.17-7.58(\mathrm{~m}, 15 \mathrm{H})$.

${ }^{13} \mathrm{C}$ NMR $\left(100 \mathrm{MHz}, \mathrm{CDCl}_{3}, 293 \mathrm{~K}\right): \delta 16.35,20.74,21.94,23.34$, 26.01, 27.94 (6C), 28.03 (3C), 28.41, 29.44, 31.25, 34.03, 35.00, $40.80,46.81,47.57,47.89,49.61,59.99,60.17,60.64,62.35,62.62$, $66.29,75.44,81.18,81.39,81.59,126.94$ (2C), 127.06 (2C), 127.94 (2C), 128.11, 128.39, 128.55 (2C), 128.57 (2C), 128.62, 128.82 (2C), 137.48, 138.77, 139.22, 167.35, 168.06, 169.60, 169.81, 171.84, 171.92 .

HRMS (EI) calculated for $\mathrm{C}_{58} \mathrm{H}_{78} \mathrm{~N}_{3} \mathrm{O}_{10}[\mathrm{M}+\mathrm{H}]^{+}$976.5682, found 976.5680 .

4-((1S,2R,5S)-2-isopropyl-5-methylcyclohexyl)

2-methyl

$(2 R, 4 R, 5 S)-1-((2 R, 3 S, 5 S)-5-$ (methoxycarbonyl)-1-((2S,3R,5R)-5-(methoxycarbonyl)-2-phenylpyrrolidine-3-carbonyl)-2phenylpyrrolidine-3-carbonyl)-5-phenylpyrrolidine-2,4-dicarboxylate D-3a

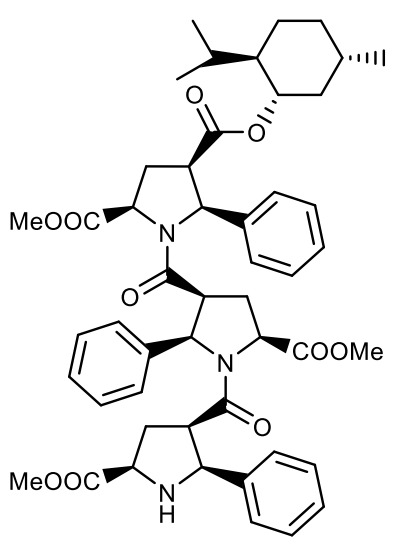

Yield $80 \%, 11.940 \mathrm{~g}$, colorless crystals, m.p. $144-146{ }^{\circ} \mathrm{C},[\alpha]_{D}{ }^{21}+19.9^{\circ}(\mathrm{c}$ $0.94, \mathrm{MeOH})$.

${ }^{1} \mathrm{H}$ NMR $\left(400 \mathrm{MHz}, \mathrm{DMSO}-\mathrm{d}_{6}, 293 \mathrm{~K}\right): \delta 0.52(\mathrm{~d}, J 6.8 \mathrm{~Hz}, 3 \mathrm{H}), 0.71-0.82$ $(\mathrm{m}, 1 \mathrm{H}), 0.77(\mathrm{~d}, J 6.8 \mathrm{~Hz}, 3 \mathrm{H}), 0.85(\mathrm{~d}, J 6.8 \mathrm{~Hz}, 3 \mathrm{H}), 0.85-0.98(\mathrm{~m}, 2 \mathrm{H})$, 1.23-1.34 (m, 2H), 1.41-1.47 (m, 1H), 1.50-1.61 (m, 4H), $1.68(\mathrm{dt}, J$ 12.5, $7.6 \mathrm{~Hz}, 1 \mathrm{H}$ ), 1.92 (ddd, J 12.5, 9.3, 7.6 Hz, 1H), 1.98-2.07 (m, 1H), 2.14$2.23(\mathrm{~m}, 1 \mathrm{H}), 2.29-2.37(\mathrm{~m}, 1 \mathrm{H}), 2.82(\mathrm{ddd}, J 11.5,8.2,6.6 \mathrm{~Hz}, 1 \mathrm{H}), 3.00-$ $3.07(\mathrm{~m}, 1 \mathrm{H}), 3.08-3.13(\mathrm{~m}, 2 \mathrm{H}), 3.53(\mathrm{~s}, 3 \mathrm{H}), 3.59(\mathrm{~s}, 3 \mathrm{H}), 3.64(\mathrm{~s}, 3 \mathrm{H})$, 3.64-3.69 (m, 1H), 3.74-3.80 (m, 2H), $4.40(\mathrm{td}, J 10.9,4.3 \mathrm{~Hz}, 1 \mathrm{H}), 4.66$ (t, J $7.6 \mathrm{~Hz}, 1 \mathrm{H}), 5.61(\mathrm{~d}, J 8.6 \mathrm{~Hz}, 1 \mathrm{H}), 5.90(\mathrm{~d}, J 8.6 \mathrm{~Hz}, 1 \mathrm{H}), 7.10-7.19$ $(\mathrm{m}, 3 \mathrm{H}), 7.20-7.30(\mathrm{~m}, 2 \mathrm{H}), 7.33-7.43(\mathrm{~m}, 6 \mathrm{H}), 7.60(\mathrm{~d}, J 7.3 \mathrm{~Hz}, 2 \mathrm{H})$,

7.72-7.75 (m, 2H).

${ }^{13} \mathrm{C}$ NMR (100 MHz, DMSO-d $\left.6,293 \mathrm{~K}\right): \delta 15.92,20.64,21.80,22.55,25.11,28.60,29.06,30.65$, $30.91,33.35,33.51,40.25,46.11,46.42$, 46.96, 48.98, 51.69, 51.81, 58.23, 58.98, 59.13, 60.47, $61.19,63.21,74.03,126.86(3 \mathrm{C}), 126.97(2 \mathrm{C}), 127.49$ (2C), $127.53(2 \mathrm{C}), 127.64,128.08(2 \mathrm{C})$, $128.17,128.40$ (2C), 138.79, 139.35, 141.04, 166.94, 168.19, 171.27, 171.57, 171.64, 173.32.

Anal. Calcd for $\mathrm{C}_{49} \mathrm{H}_{59} \mathrm{~N}_{3} \mathrm{O}_{10}$ : C 69.24, H 7.00, N 4.94. Found: C 69.56, H 6.87, N 4.87. 
4-((1R,2S,5R)-2-isopropyl-5-methylcyclohexyl) 2-methyl $\quad(2 S, 4 S, 5 R)-5$-(3-chlorophenyl)-1$((2 S, 3 R, 5 R)-5$-(methoxycarbonyl)-1-((2R,3S,5S)-5-(methoxycarbonyl)-2-phenylpyrrolidine-3carbonyl)-2-(4-methoxyphenyl)pyrrolidine-3-carbonyl)pyrrolidine-2,4-dicarboxylate L-3d

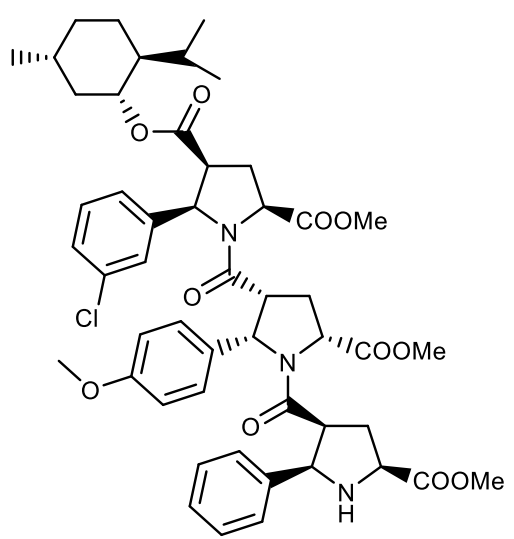
Yield $87 \%, 0.430 \mathrm{~g}$, colorless crystals, m.p. $130-132{ }^{\circ} \mathrm{C},[\alpha]_{D}{ }^{21}$ $+12.3^{\circ}$ (c 1.13, $\mathrm{MeOH}$ ).

${ }^{1} \mathrm{H}$ NMR $\left(400 \mathrm{MHz}, \mathrm{DMSO}-\mathrm{d}_{6}, 293 \mathrm{~K}\right): \delta 0.54(\mathrm{~d}, J 6.8 \mathrm{~Hz}, 3 \mathrm{H})$, 0.74-0.82 (m, $1 \mathrm{H}), 0.78(\mathrm{~d}, J 6.8 \mathrm{~Hz}, 3 \mathrm{H}), 0.85(\mathrm{~d}, J 6.8 \mathrm{~Hz}, 3 \mathrm{H})$, 0.85-0.97 (m, 2H), 1.28-1.34 (m, 2H), 1.43-1.51 (m, 1H), 1.56$1.63(\mathrm{~m}, 4 \mathrm{H}), 1.70(\mathrm{dt}, J 12.4,7.6 \mathrm{~Hz}, 1 \mathrm{H}), 1.92(\mathrm{ddd}, J 12.5,9.3$, $7.6 \mathrm{~Hz}, 1 \mathrm{H}), 1.98-2.07(\mathrm{~m}, 1 \mathrm{H}), 2.10-2.18(\mathrm{~m}, 1 \mathrm{H}), 2.31-2.37(\mathrm{~m}$, $1 \mathrm{H}), 2.69-2.76(\mathrm{~m}, 1 \mathrm{H}), 2.93-3.04(\mathrm{~m}, 1 \mathrm{H}), 3.10-3.16(\mathrm{~m}, 2 \mathrm{H})$, $3.52(\mathrm{~s}, 3 \mathrm{H}), 3.59(\mathrm{~s}, 3 \mathrm{H}), 3.63(\mathrm{~s}, 3 \mathrm{H}), 3.64-3.69(\mathrm{~m}, 1 \mathrm{H}), 3.74-$ $3.80(\mathrm{~m}, 2 \mathrm{H}), 3.75(\mathrm{~s}, 3 \mathrm{H}), 4.42(\mathrm{td}, J 10.9,4.2 \mathrm{~Hz}, 1 \mathrm{H}), 4.63(\mathrm{~d}, J$ $7.8 \mathrm{~Hz}, 1 \mathrm{H}), 5.54(\mathrm{~d}, J 8.4 \mathrm{~Hz}, 1 \mathrm{H}), 5.88(\mathrm{~d}, J 8.4 \mathrm{~Hz}, 1 \mathrm{H}), 6.90$ (d, J 8.6 Hz, 2H), 7.10-7.18 (m, 3H), 7.24-7.29 (m, 1H), 7.31-7.33 (m, 2H), 7.45-7.46 (m, $1 \mathrm{H}), 7.50(\mathrm{~d}, J$ $8.6 \mathrm{~Hz}, 2 \mathrm{H}), 7.75-7.77(\mathrm{~m}, 2 \mathrm{H})$.

${ }^{13} \mathrm{C}$ NMR (100 MHz, DMSO-d $\left.6,293 \mathrm{~K}\right): \delta$ 16.01, 20.72, 21.94, 22.70, 25.40, 28.67, 29.11, 30.82, $33.55,33.66,40.40,46.25,46.48,47.16,49.05,51.80,52.04,55.02,58.33,59.16,59.28,60.20$, $60.68,63.45,64.98,74.43,113.59$ (2C), 126.07, 126.90 (2C), 126.93, 127.51, 127.61 (2C), 128.26 (2C), 128.48, 130.56, 131.22, 133.42, 141.01, 141.37, 158.69, 167.26, 168.27, 171.48, $171.76,171.92,173.44$.

HRMS (EI) calculated for $\mathrm{C}_{50} \mathrm{H}_{61} \mathrm{ClN}_{3} \mathrm{O}_{11}[\mathrm{M}+\mathrm{H}]^{+}$914.3995, found 914.4044.

4-((1S,2R,5S)-2-isopropyl-5-methylcyclohexyl) 2-methyl $\quad(2 R, 4 R, 5 S)-5$-(3-chlorophenyl)-1-

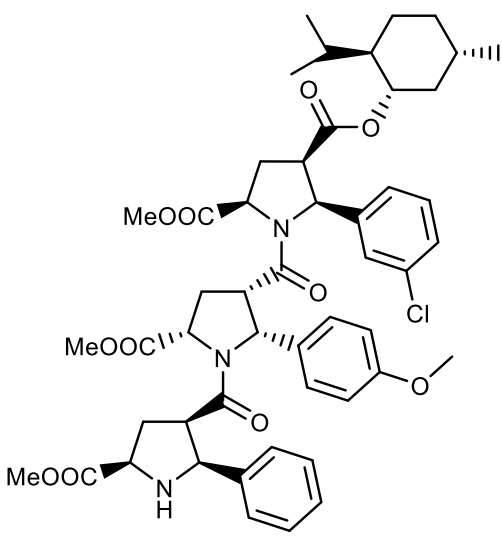
((2R,3S,5S)-5-(methoxycarbonyl)-1-((2S,3R,5R)-5-

(methoxycarbonyl)-2-phenylpyrrolidine-3-carbonyl)-2-(4methoxyphenyl)pyrrolidine-3-carbonyl)pyrrolidine-2,4dicarboxylate D-3d

Yield $79 \%, 0.459 \mathrm{~g}$, colorless crystals, m.p. $130-132{ }^{\circ} \mathrm{C},[\alpha]_{D}{ }^{21}$ $-11.5^{\circ}$ (c 1.13, MeOH).

${ }^{1} \mathrm{H}$ NMR $\left(400 \mathrm{MHz}, \mathrm{DMSO}-\mathrm{d}_{6}, 293 \mathrm{~K}\right): \delta 0.54(\mathrm{~d}, J 6.8 \mathrm{~Hz}, 3 \mathrm{H})$, 0.74-0.82 (m, $1 \mathrm{H}), 0.78(\mathrm{~d}, J 6.8 \mathrm{~Hz}, 3 \mathrm{H}), 0.85(\mathrm{~d}, J 6.8 \mathrm{~Hz}, 3 \mathrm{H})$, 0.85-0.97 (m, 2H), 1.28-1.34 (m, 2H), 1.43-1.51 (m, 1H), 1.56$1.63(\mathrm{~m}, 4 \mathrm{H}), 1.70(\mathrm{dt}, J 12.4,7.6 \mathrm{~Hz}, 1 \mathrm{H}), 1.92$ (ddd, J 12.5, 9.3, $7.6 \mathrm{~Hz}, 1 \mathrm{H}), 1.98-2.07(\mathrm{~m}, 1 \mathrm{H}), 2.10-2.18(\mathrm{~m}, 1 \mathrm{H}), 2.31-2.37(\mathrm{~m}$, $1 \mathrm{H}), 2.69-2.76(\mathrm{~m}, 1 \mathrm{H}), 2.93-3.04(\mathrm{~m}, 1 \mathrm{H}), 3.10-3.16(\mathrm{~m}, 2 \mathrm{H}), 3.52(\mathrm{~s}, 3 \mathrm{H}), 3.59(\mathrm{~s}, 3 \mathrm{H}), 3.63(\mathrm{~s}$, $3 \mathrm{H}), 3.64-3.69(\mathrm{~m}, 1 \mathrm{H}), 3.74-3.80(\mathrm{~m}, 2 \mathrm{H}), 3.75(\mathrm{~s}, 3 \mathrm{H}), 4.42(\mathrm{td}, J 10.9,4.2 \mathrm{~Hz}, 1 \mathrm{H}), 4.63(\mathrm{~d}, J 7.8$ $\mathrm{Hz}, 1 \mathrm{H}), 5.54(\mathrm{~d}, J 8.4 \mathrm{~Hz}, 1 \mathrm{H}), 5.88(\mathrm{~d}, J 8.4 \mathrm{~Hz}, 1 \mathrm{H}), 6.90(\mathrm{~d}, J 8.6 \mathrm{~Hz}, 2 \mathrm{H}), 7.10-7.18(\mathrm{~m}, 3 \mathrm{H})$, 7.24-7.29 (m, 1H), 7.31-7.33 (m, 2H), 7.45-7.46 (m, $1 \mathrm{H}), 7.50(\mathrm{~d}, J 8.6 \mathrm{~Hz}, 2 \mathrm{H}), 7.75-7.77(\mathrm{~m}, 2 \mathrm{H})$. ${ }^{13} \mathrm{C}$ NMR (100 MHz, DMSO-d6, $\left.293 \mathrm{~K}\right): \delta$ 16.01, 20.72, 21.94, 22.70, 25.40, 28.67, 29.11, 30.82, 33.55, 33.66, 40.40, 46.25, 46.48, 47.16, 49.05, 51.80, 52.04, 55.02, 58.33, 59.16, 59.28, 60.20, $60.68,63.45,64.98,74.43,113.59$ (2C), 126.07, 126.90 (2C), 126.93, 127.51, 127.61 (2C), 128.26 (2C), 128.48, 130.56, 131.22, 133.42, 141.01, 141.37, 158.69, 167.26, 168.27, 171.48, $171.76,171.92,173.44$.

HRMS (EI) calculated for $\mathrm{C}_{50} \mathrm{H}_{61} \mathrm{ClN}_{3} \mathrm{O}_{11}[\mathrm{M}+\mathrm{H}]^{+}$914.3995, found 914.4044. 
4-((1R,2S,5R)-2-isopropyl-5-methylcyclohexyl) 2-methyl $\quad(2 S, 4 S, 5 R)-1-((2 S, 3 R, 5 R)-1-((2 R, 3 S, 5 S)-$ 1-acryloyl-5-(methoxycarbonyl)-2-phenylpyrrolidine-3-carbonyl)-5-(methoxycarbonyl)-2phenylpyrrolidine-3-carbonyl)-5-phenylpyrrolidine-2,4-dicarboxylate L-3a_A

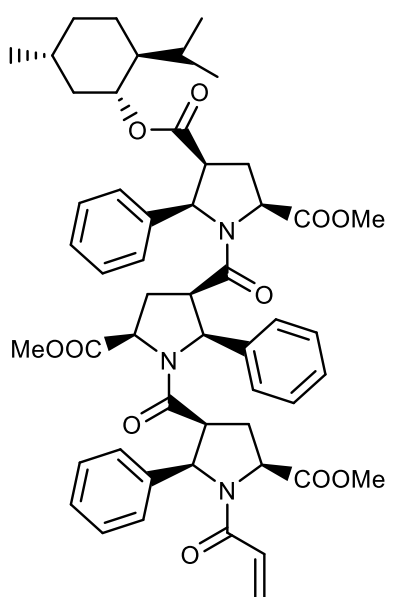

Yield $85 \%, 1.000 \mathrm{~g}$, colorless crystals, m.p. $169-171{ }^{\circ} \mathrm{C},[\alpha]_{D}{ }^{21}-57.5^{\circ}(\mathrm{c}$ $0.445, \mathrm{MeOH})$.

${ }^{1} \mathrm{H}$ NMR $(400 \mathrm{MHz}$, DMSO-d $6,293 \mathrm{~K}): \delta 0.51(\mathrm{~d}, J 6.8 \mathrm{~Hz}, 3 \mathrm{H}), 0.72-0.81$ $(\mathrm{m}, 1 \mathrm{H}), 0.76(\mathrm{~d}, J 6.8 \mathrm{~Hz}, 3 \mathrm{H}), 0.82(\mathrm{~d}, J 6.8 \mathrm{~Hz}, 3 \mathrm{H}), 0.84-0.95(\mathrm{~m}, 2 \mathrm{H})$, $1.20-1.34(\mathrm{~m}, 3 \mathrm{H}), 1.39-1.49(\mathrm{~m}, 1 \mathrm{H}), 1.54-1.60(\mathrm{~m}, 3 \mathrm{H}), 1.76-1.83(\mathrm{~m}$, $1 \mathrm{H}), 1.93-1.99(\mathrm{~m}, 1 \mathrm{H}), 2.02-2.12(\mathrm{~m}, 1 \mathrm{H}), 2.19-2.29(\mathrm{~m}, 2 \mathrm{H}), 2.33-2.40$ $(\mathrm{m}, 1 \mathrm{H}), 2.98-3.05(\mathrm{~m}, 1 \mathrm{H}), 3.17-3.34(\mathrm{~m}, 2 \mathrm{H}), 3.51(\mathrm{~s}, 3 \mathrm{H}), 3.60-3.72$ $(\mathrm{m}, 1 \mathrm{H}), 3.63(\mathrm{~s}, 3 \mathrm{H}), 3.69(\mathrm{~s}, 3 \mathrm{H}), 3.85-3.90(\mathrm{~m}, 1 \mathrm{H}), 4.22(\mathrm{dd}, J 11.5$, $6.6 \mathrm{~Hz}, 1 \mathrm{H}), 4.42(\mathrm{td}, J 10.8,4.2 \mathrm{~Hz}, 1 \mathrm{H}), 5.64(\mathrm{dd}, J$ 8.0, $4.2 \mathrm{~Hz}, 1 \mathrm{H})$, 5.96-5-98 (m, 2H), 6.16-6.21 (m, 2H), 7.03-7.07 (m, 2H), 7.16-7.21 (m, $2 \mathrm{H}), 7.27-7.41(\mathrm{~m}, 7 \mathrm{H}), 7.65(\mathrm{~d}, J 7.3 \mathrm{~Hz}, 2 \mathrm{H}), 7.79-7.81(\mathrm{~m}, 2 \mathrm{H})$.

Anal. Calcd for $\mathrm{C}_{52} \mathrm{H}_{61} \mathrm{~N}_{3} \mathrm{O}_{11}$ : C 69.08, $\mathrm{H} 6.80, \mathrm{~N}$ 4.65. Found: $\mathrm{C} 68.79, \mathrm{H}$ 6.67, N 4.58. HRMS (EI) calculated for $\mathrm{C}_{52} \mathrm{H}_{62} \mathrm{~N}_{3} \mathrm{O}_{11}[\mathrm{M}+\mathrm{H}]^{+} 904.4379$, found 904.4382 .

4-((1R,2S,5R)-2-isopropyl-5-methylcyclohexyl) 2-methyl $\quad(2 S, 4 S, 5 R)-1-((2 S, 3 R, 5 R)-1-((2 R, 3 S, 5 S)$ -

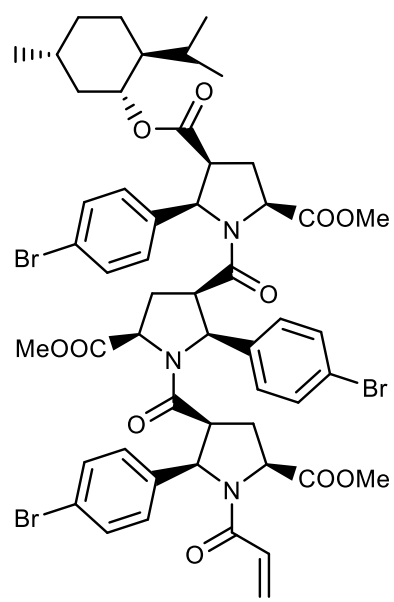
1-acryloyl-2-(4-bromophenyl)-5-(methoxycarbonyl)pyrrolidine-3carbonyl)-2-(4-bromophenyl)-5-(methoxycarbonyl)pyrrolidine-3carbonyl)-5-(4-bromophenyl)pyrrolidine-2,4-dicarboxylate L-3b_A

Yield $86 \%, 2.000 \mathrm{~g}$, colorless crystals, m.p. $175-177^{\circ} \mathrm{C},[\alpha]_{D^{21}}-22.0^{\circ}(\mathrm{c}$ $0.66, \mathrm{MeOH})$.

Anal. Calcd for $\mathrm{C}_{52} \mathrm{H}_{58} \mathrm{Br}_{3} \mathrm{~N}_{3} \mathrm{O}_{11}$ : C 54.75, H 5.13, N 3.68. Found: $\mathrm{C} 54.60$, H 5.28, N 3.37 .

HRMS (ESI) calculated for $\mathrm{C}_{52} \mathrm{H}_{59} \mathrm{Br}_{3} \mathrm{~N}_{3} \mathrm{O}_{11}[\mathrm{M}+\mathrm{H}]^{+}$1138.1694, found 1138.1702 .

2-(tert-butyl) 4-((1R,2S,5R)-2-isopropyl-5-methylcyclohexyl) $(2 S, 4 S, 5 R)-1-((2 S, 3 R, 5 R)-1$ -

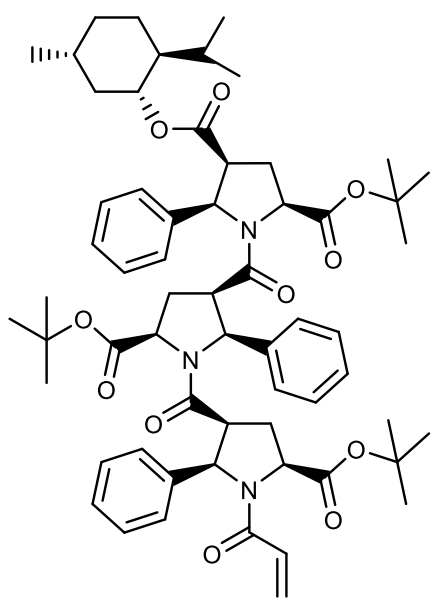

$((2 R, 3 S, 5 S)$-1-acryloyl-5-(tert-butoxycarbonyl)-2-phenylpyrrolidine3-carbonyl)-5-(tert-butoxycarbonyl)-2-phenylpyrrolidine-3carbonyl)-5-phenylpyrrolidine-2,4-dicarboxylate L-3c_A Yield $85 \%, 3.500 \mathrm{~g}$, colorless crystals, m.p. $144-146^{\circ} \mathrm{C},[\alpha]_{D}{ }^{21}-39.3^{\circ}$ (c $0.585, \mathrm{MeOH}$ ).

${ }^{1} \mathrm{H}$ NMR $(600 \mathrm{MHz}$, DMSO-d $6,293 \mathrm{~K}): \delta 0.54(\mathrm{~d}, J 7.3 \mathrm{~Hz}, 3 \mathrm{H}), 0.74-$ $0.95(\mathrm{~m}, 10 \mathrm{H}), 1.31(\mathrm{~s}, 9 \mathrm{H}), 1.39(\mathrm{~s}, 9 \mathrm{H}), 1.44(\mathrm{~s}, 9 \mathrm{H}), 1.48-1.61(\mathrm{~m}$, $6 \mathrm{H}), 1.78-1.80(\mathrm{~m}, 1 \mathrm{H}), 1.93-1.95(\mathrm{~m}, 1 \mathrm{H}), 2.02-2.06(\mathrm{~m}, 1 \mathrm{H}), 2.18-$ $2.28(\mathrm{~m}, 2 \mathrm{H}), 2.33-2.35(\mathrm{~m}, 1 \mathrm{H}), 2.96-2.98(\mathrm{~m}, 1 \mathrm{H}), 3.09-3.14(\mathrm{~m}$, $2 \mathrm{H}), 3.82-3.86(\mathrm{~m}, 1 \mathrm{H}), 3.95(\mathrm{dd}, J 11.0,6.6 \mathrm{~Hz}, 1 \mathrm{H}), 4.04(\mathrm{dd}, J$ 11.7, $5.9 \mathrm{~Hz}, 1 \mathrm{H}), 4.42-4.46(\mathrm{~m}, 1 \mathrm{H}), 5.61(\mathrm{~d}, J 8.8 \mathrm{~Hz}, 1 \mathrm{H}), 5.96(\mathrm{~d}, J$ $8.8 \mathrm{~Hz}, 2 \mathrm{H}), 6.14-6.21(\mathrm{~m}, 2 \mathrm{H}), 7.04-7.07(\mathrm{~m}, 2 \mathrm{H}), 7.12-7.21(\mathrm{~m}$, $3 \mathrm{H}), 7.25-7.33(\mathrm{~m}, 2 \mathrm{H}), 7.37-7.44(\mathrm{~m}, 4 \mathrm{H}), 7.65(\mathrm{~d}, J 7.3 \mathrm{~Hz}, 2 \mathrm{H})$,

7.80 (d, J $7.3 \mathrm{~Hz}, 2 \mathrm{H})$.

HRMS (ESI) calculated for $\mathrm{C}_{61} \mathrm{H}_{80} \mathrm{~N}_{3} \mathrm{O}_{11}[\mathrm{M}+\mathrm{H}]^{+} 1030.5787$, found 1030.5821 . 
4-((1S,2R,5S)-2-isopropyl-5-methylcyclohexyl) 2-methyl $\quad(2 R, 4 R, 5 S)-1-((2 R, 3 S, 5 S)-1-((2 S, 3 R, 5 R)-$ 1-acryloyl-5-(methoxycarbonyl)-2-phenylpyrrolidine-3-carbonyl)-5-(methoxycarbonyl)-2-

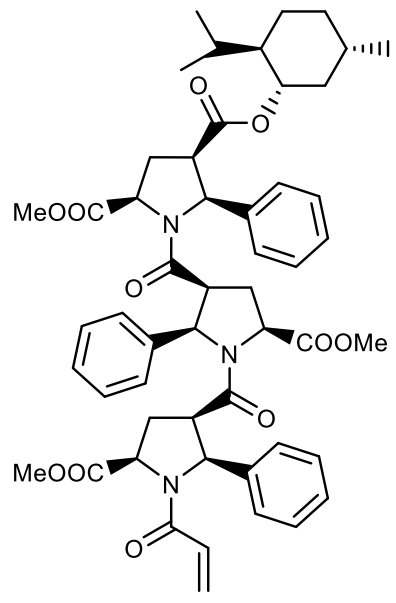

phenylpyrrolidine-3-carbonyl)-5-phenylpyrrolidine-2,4-dicarboxylate

D-3a_A

Yield $84 \%, 8.800 \mathrm{~g}$, colorless crystals, m.p. $169-171^{\circ} \mathrm{C},[\alpha]_{D}^{21}+57.1^{\circ}(c$ $0.515, \mathrm{MeOH})$.

${ }^{1} \mathrm{H}$ NMR $\left(400 \mathrm{MHz}, \mathrm{DMSO}-\mathrm{d}_{6}, 293 \mathrm{~K}\right): \delta 0.51(\mathrm{~d}, J 6.8 \mathrm{~Hz}, 3 \mathrm{H}), 0.72$ $0.81(\mathrm{~m}, 1 \mathrm{H}), 0.76(\mathrm{~d}, J 6.8 \mathrm{~Hz}, 3 \mathrm{H}), 0.82(\mathrm{~d}, J 6.8 \mathrm{~Hz}, 3 \mathrm{H}), 0.84-0.95$ $(\mathrm{m}, 2 \mathrm{H}), 1.20-1.34(\mathrm{~m}, 3 \mathrm{H}), 1.39-1.49(\mathrm{~m}, 1 \mathrm{H}), 1.54-1.60(\mathrm{~m}, 3 \mathrm{H})$, 1.76-1.83 $(\mathrm{m}, 1 \mathrm{H}), 1.93-1.99(\mathrm{~m}, 1 \mathrm{H}), 2.02-2.12(\mathrm{~m}, 1 \mathrm{H}), 2.19-2.29(\mathrm{~m}$, $2 \mathrm{H}), 2.33-2.40(\mathrm{~m}, 1 \mathrm{H}), 2.98-3.05(\mathrm{~m}, 1 \mathrm{H}), 3.17-3.34(\mathrm{~m}, 2 \mathrm{H}), 3.51(\mathrm{~s}$, $3 \mathrm{H}), 3.60-3.72(\mathrm{~m}, 1 \mathrm{H}), 3.63(\mathrm{~s}, 3 \mathrm{H}), 3.69(\mathrm{~s}, 3 \mathrm{H}), 3.85-3.90(\mathrm{~m}, 1 \mathrm{H})$, $4.22(\mathrm{dd}, J 11.5,6.6 \mathrm{~Hz}, 1 \mathrm{H}), 4.42(\mathrm{td}, J 10.8,4.2 \mathrm{~Hz}, 1 \mathrm{H}), 5.64$ (dd, J 8.0, 4.2 Hz, $1 \mathrm{H}), 5.96-5-98(\mathrm{~m}, 2 \mathrm{H}), 6.16-6.21(\mathrm{~m}, 2 \mathrm{H}), 7.03-7.07(\mathrm{~m}$, $2 \mathrm{H}), 7.16-7.21(\mathrm{~m}, 2 \mathrm{H}), 7.27-7.41(\mathrm{~m}, 7 \mathrm{H}), 7.65(\mathrm{~d}, J 7.3 \mathrm{~Hz}, 2 \mathrm{H}), 7.79-7.81(\mathrm{~m}, 2 \mathrm{H})$. HRMS (EI) calculated for $\mathrm{C}_{52} \mathrm{H}_{62} \mathrm{~N}_{3} \mathrm{O}_{11}[\mathrm{M}+\mathrm{H}]^{+} 904.4379$, found 904.4382 .

4-((1R,2S,5R)-2-isopropyl-5-methylcyclohexyl) 2-methyl $\quad(2 S, 4 S, 5 R)-1-((2 S, 3 R, 5 R)-5-$

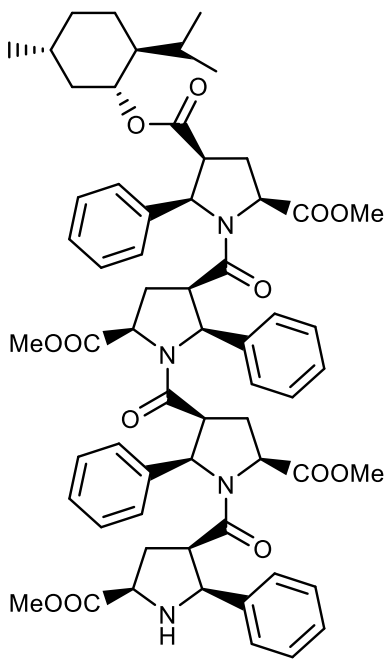
(methoxycarbonyl)-1-((2R,3S,5S)-5-(methoxycarbonyl)-1-((2S,3R,5R)-5(methoxycarbonyl)-2-phenylpyrrolidine-3-carbonyl)-2phenylpyrrolidine-3-carbonyl)-2-phenylpyrrolidine-3-carbonyl)-5phenylpyrrolidine-2,4-dicarboxylate L-4a

Yield $85 \%, 0.950 \mathrm{~g}$, colorless crystals, m.p. $179-181^{\circ} \mathrm{C},[\alpha]_{D}{ }^{21}-18.0^{\circ}$ (c $0.25, \mathrm{MeOH})$.

HRMS (ESI) calculated for $\mathrm{C}_{62} \mathrm{H}_{72} \mathrm{~N}_{4} \mathrm{NaO}_{13}[\mathrm{M}+\mathrm{Na}]^{+} 1103.4988$, found 1103.4998.

4-((1R,2S,5R)-2-isopropyl-5-methylcyclohexyl) 2-methyl $\quad(2 S, 4 S, 5 R)-5$-(4-bromophenyl)-1-

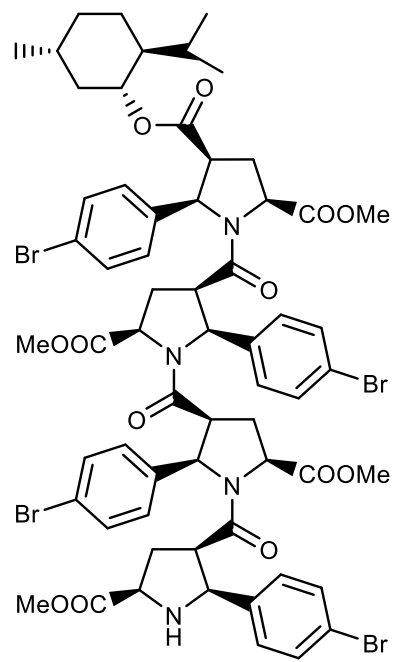
((2S,3R,5R)-2-(4-bromophenyl)-1-((2R,3S,5S)-2-(4-bromophenyl)-1((2S,3R,5R)-2-(4-bromophenyl)-5-(methoxycarbonyl)pyrrolidine-3carbonyl)-5-(methoxycarbonyl)pyrrolidine-3-carbonyl)-5(methoxycarbonyl)pyrrolidine-3-carbonyl)pyrrolidine-2,4dicarboxylate L-4b

Yield $86 \%, 1.980 \mathrm{~g}$, colorless crystals, m.p. $228-230{ }^{\circ} \mathrm{C},[\alpha]_{D}{ }^{21}+7.2^{\circ}(\mathrm{c}$ 1.13, $\left.\mathrm{CHCl}_{3}\right)$.

Anal. Calcd for $\mathrm{C}_{62} \mathrm{H}_{68} \mathrm{Br}_{4} \mathrm{~N}_{4} \mathrm{O}_{13}$ : $\mathrm{C} 53.31, \mathrm{H}$ 4.91, N 4.01. Found: $\mathrm{C} 53.56$, H 5.28, N 3.95 .

HRMS (ESI) calculated for $\mathrm{C}_{62} \mathrm{H}_{69} \mathrm{Br}_{4} \mathrm{~N}_{4} \mathrm{O}_{13}[\mathrm{M}+\mathrm{H}]^{+}$1393.1589, found 1393.1663. 
2-(tert-butyl) $\quad 4-((1 R, 2 S, 5 R)$-2-isopropyl-5-methylcyclohexyl) $\quad(2 S, 4 S, 5 R)-1-((2 S, 3 R, 5 R)-5$-(tertbutoxycarbonyl)-1-((2R,3S,5S)-5-(tert-butoxycarbonyl)-1-((2S,3R,5R)-5-(tert-butoxycarbonyl)-2-

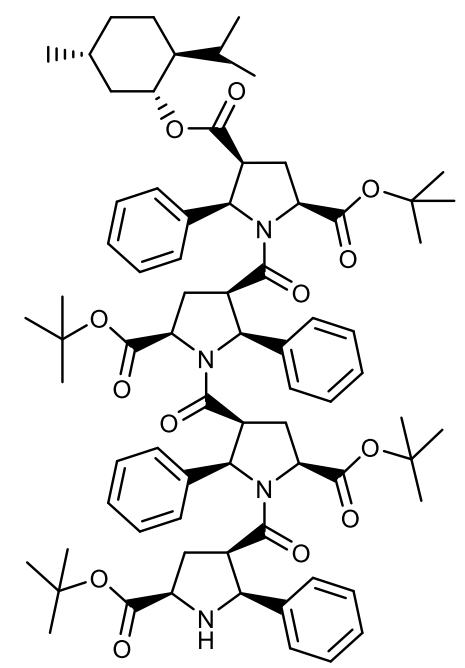
phenylpyrrolidine-3-carbonyl)-2-phenylpyrrolidine-3-carbonyl)-2phenylpyrrolidine-3-carbonyl)-5-phenylpyrrolidine-2,4dicarboxylate L-4C

Yield $81 \%, 3.870 \mathrm{~g}$, colorless crystals, m.p. $150-152^{\circ} \mathrm{C},[\alpha]_{D}{ }^{21}-23.4^{\circ}$ (c $0.535, \mathrm{MeOH})$.

${ }^{1} \mathrm{H}$ NMR $\left(600 \mathrm{MHz}, \mathrm{DMSO}-\mathrm{d}_{6}, 293 \mathrm{~K}\right): \delta 0.52(\mathrm{~d}, J 7.3 \mathrm{~Hz}, 3 \mathrm{H}), 0.58$ (d, J $7.3 \mathrm{~Hz}, 3 \mathrm{H}), 0.73-0.80(\mathrm{~m}, 3 \mathrm{H}), 0.83(\mathrm{~d}, J 7.3 \mathrm{~Hz}, 3 \mathrm{H}), 0.88-0.98$ $(\mathrm{m}, 3 \mathrm{H}), 1.30(\mathrm{~s}, 9 \mathrm{H}), 1.32-1.37(\mathrm{~m}, 3 \mathrm{H}), 1.38-1.42(\mathrm{~m}, 28 \mathrm{H}), 1.61-$ $1.65(\mathrm{~m}, 1 \mathrm{H}), 1.79-1.83(\mathrm{~m}, 1 \mathrm{H}), 1.84-1.87(\mathrm{~m}, 1 \mathrm{H}), 1.92-1.95(\mathrm{~m}$, $1 \mathrm{H}), 1.97-2.00(\mathrm{~m}, 1 \mathrm{H}), 2.06-2.11(\mathrm{~m}, 1 \mathrm{H}), 2.15-2.21(\mathrm{~m}, 1 \mathrm{H}), 2.30-$ $2.37(\mathrm{~m}, 1 \mathrm{H}), 2.81(\mathrm{t}, J 10.3 \mathrm{~Hz}, 1 \mathrm{H}), 2.91-2.94(\mathrm{~m}, 1 \mathrm{H}), 3.07-3.11$ (m, $1 \mathrm{H}), 3.16-3.19(\mathrm{~m}, 1 \mathrm{H}), 3.29(\mathrm{dd}, J 10.3,7.3 \mathrm{~Hz}, 1 \mathrm{H}), 3.59-3.63$ (m, $1 \mathrm{H}), 3.85-3.93(\mathrm{~m}, 2 \mathrm{H}), 4.38(\mathrm{dd}, J 10.3,7.3 \mathrm{~Hz}, 1 \mathrm{H}), 4.41-4.45$ $(\mathrm{m}, 1 \mathrm{H}), 4.73(\mathrm{~d}, J 8.8 \mathrm{~Hz}, 1 \mathrm{H}), 5.33(\mathrm{~d}, J 8.8 \mathrm{~Hz}, 1 \mathrm{H}), 5.54(\mathrm{~d}, J 8.8$ $\mathrm{Hz}, 1 \mathrm{H}), 7.18-7.20(\mathrm{~m}, 2 \mathrm{H}), 7.26-7.52(\mathrm{~m}, 14 \mathrm{H}), 7.55(\mathrm{~d}, J 7.3 \mathrm{~Hz}, 2 \mathrm{H}), 7.78(\mathrm{~d}, J 5.9 \mathrm{~Hz}, 2 \mathrm{H})$. HRMS (ESI) calculated for $\mathrm{C}_{74} \mathrm{H}_{97} \mathrm{~N}_{4} \mathrm{O}_{13}[\mathrm{M}+\mathrm{H}]^{+} 1249.6974$, found 1249.7102 .

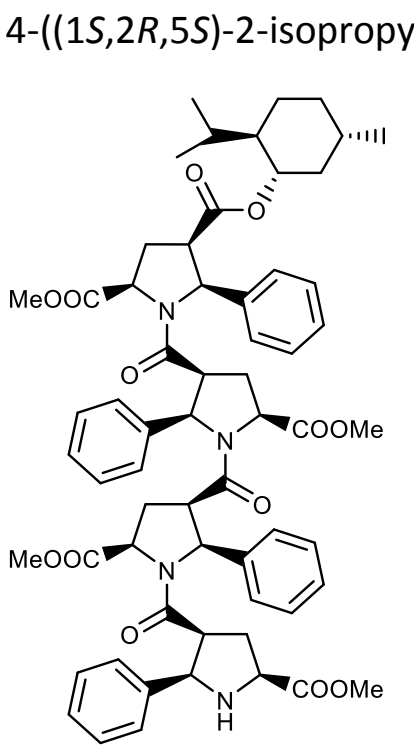

2-methyl

$(2 R, 4 R, 5 S)-1-((2 R, 3 S, 5 S)-5-$ (methoxycarbonyl)-1-((2S,3R,5R)-5-(methoxycarbonyl)-1-((2R,3S,5S)-5(methoxycarbonyl)-2-phenylpyrrolidine-3-carbonyl)-2phenylpyrrolidine-3-carbonyl)-2-phenylpyrrolidine-3-carbonyl)-5phenylpyrrolidine-2,4-dicarboxylate D-4a

Yield $82 \%, 9.000$ g, colorless crystals, m.p. $179-181^{\circ} \mathrm{C},[\alpha]_{D}{ }^{21}+18.8^{\circ}$ (c $0.67, \mathrm{MeOH})$.

Anal. Calcd for $\mathrm{C}_{62} \mathrm{H}_{72} \mathrm{~N}_{4} \mathrm{O}_{13}$ : C 68.87, H 6.71, N 5.18. Found: $68.77, \mathrm{H}$ 6.69, N 4.96.

HRMS (ESI) calculated for $\mathrm{C}_{62} \mathrm{H}_{72} \mathrm{~N}_{4} \mathrm{NaO}_{13}[\mathrm{M}+\mathrm{Na}]^{+} 1103.4988$, found 1103.4998.

4-((1R,2S,5R)-2-isopropyl-5-methylcyclohexyl) 2-methyl $(2 S, 4 S, 5 R)-1-((2 S, 3 R, 5 R)-1-((2 R, 3 S, 5 S)-$

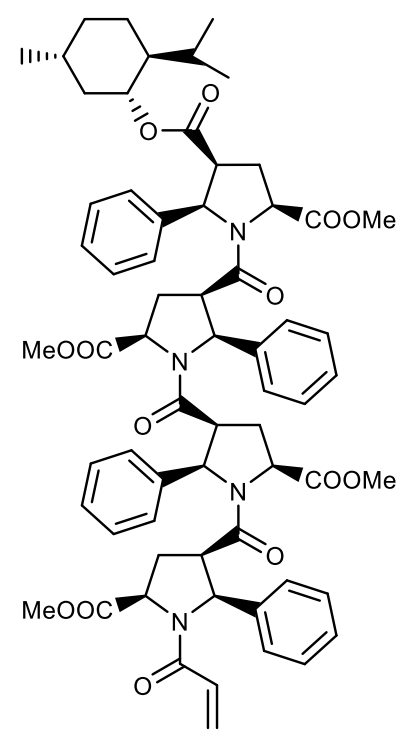
1-((2S,3R,5R)-1-acryloyl-5-(methoxycarbonyl)-2-phenylpyrrolidine-3carbonyl)-5-(methoxycarbonyl)-2-phenylpyrrolidine-3-carbonyl)-5(methoxycarbonyl)-2-phenylpyrrolidine-3-carbonyl)-5phenylpyrrolidine-2,4-dicarboxylate L-4a_A

Yield $82 \%, 0.680$ g, colorless crystals, m.p. $194-196^{\circ} \mathrm{C},[\alpha]_{D}{ }^{21}+36.7^{\circ}(\mathrm{c}$ $0.30, \mathrm{MeOH})$.

HRMS (ESI) calculated for $\mathrm{C}_{65} \mathrm{H}_{74} \mathrm{~N}_{4} \mathrm{NaO}_{14}[\mathrm{M}+\mathrm{Na}]^{+} 1157.5106$, found 1157.5106 . 
4-((1R,2S,5R)-2-isopropyl-5-methylcyclohexyl) 2-methyl $\quad(2 S, 4 S, 5 R)-1-((2 S, 3 R, 5 R)-1-((2 R, 3 S, 5 S)$ -<smiles>C=CC(=O)N1[C@H](c2ccc(Br)cc2)C[C@H](C(C)=O)[C@H]1C(=O)N1[C@H](C(C)=O)C[C@H](c2ccc(Br)cc2)[C@H]1C(=O)N1[C@H](C(C)=O)CC[C@H]1c1ccc(Br)cc1</smiles1-((2S,3R,5R)-1-acryloyl-2-(4-bromophenyl)-5(methoxycarbonyl)pyrrolidine-3-carbonyl)-2-(4-bromophenyl)-5(methoxycarbonyl)pyrrolidine-3-carbonyl)-2-(4-bromophenyl)-5(methoxycarbonyl)pyrrolidine-3-carbonyl)-5-(4bromophenyl)pyrrolidine-2,4-dicarboxylate L-4b_A

Yield $80 \%, 1.100$ g, colorless crystals, m.p. $220-222^{\circ} \mathrm{C}$.

Anal. Calcd for $\mathrm{C}_{65} \mathrm{H}_{70} \mathrm{Br}_{4} \mathrm{~N}_{4} \mathrm{O}_{14}$ : C 53.81, $\mathrm{H}$ 4.86, N 3.86. Found: C 53.56, H 5.20, N 3.95.

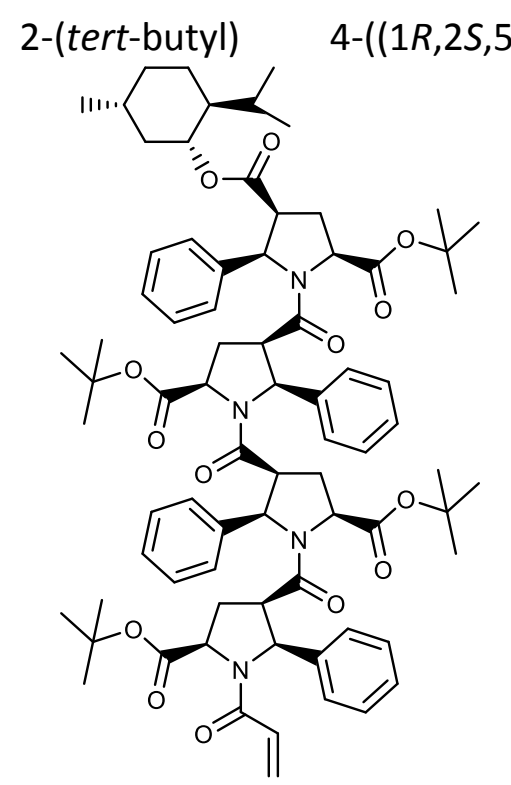

$(2 S, 4 S, 5 R)-1-((2 S, 3 R, 5 R)-1-$ $((2 R, 3 S, 5 S)-1-((2 S, 3 R, 5 R)$-1-acryloyl-5-(tert-butoxycarbonyl)-2phenylpyrrolidine-3-carbonyl)-5-(tert-butoxycarbonyl)-2phenylpyrrolidine-3-carbonyl)-5-(tert-butoxycarbonyl)-2phenylpyrrolidine-3-carbonyl)-5-phenylpyrrolidine-2,4dicarboxylate L-4c_A

Yield $75 \%, 3.510 \mathrm{~g}$, colorless crystals, m.p. $178-180^{\circ} \mathrm{C},[\alpha]_{D}{ }^{21}$ $-32.8^{\circ}\left(c 0.355, \mathrm{CH}_{2} \mathrm{Cl}_{2}\right.$ ).

HRMS (ESI) calculated for $\mathrm{C}_{77} \mathrm{H}_{99} \mathrm{~N}_{4} \mathrm{O}_{14}[\mathrm{M}+\mathrm{H}]^{+} 1303.7152$, found 1303.7211.

4-((1S,2R,5S)-2-isopropyl-5-methylcyclohexyl) 2-methyl $\quad(2 R, 4 R, 5 S)-1-((2 R, 3 S, 5 S)-1-((2 S, 3 R, 5 R)-$

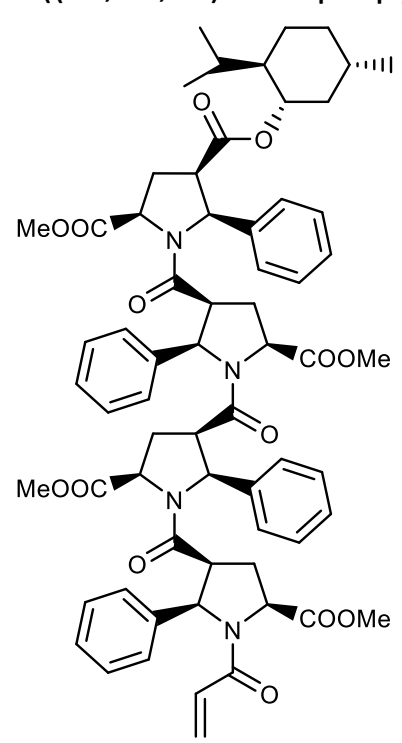
1-((2R,3S,5S)-1-acryloyl-5-(methoxycarbonyl)-2-phenylpyrrolidine-3carbonyl)-5-(methoxycarbonyl)-2-phenylpyrrolidine-3-carbonyl)-5(methoxycarbonyl)-2-phenylpyrrolidine-3-carbonyl)-5phenylpyrrolidine-2,4-dicarboxylate D-4a_A

Yield $85 \%, 4.300$ g, colorless crystals, m.p. $194-196{ }^{\circ} \mathrm{C},[\alpha]_{D}{ }^{21}-37.1^{\circ}(c$ $0.52, \mathrm{MeOH})$.

HRMS (ESI) calculated for $\mathrm{C}_{65} \mathrm{H}_{74} \mathrm{~N}_{4} \mathrm{NaO}_{14}[\mathrm{M}+\mathrm{Na}]^{+} 1157.5106$, found 1157.5094 . 


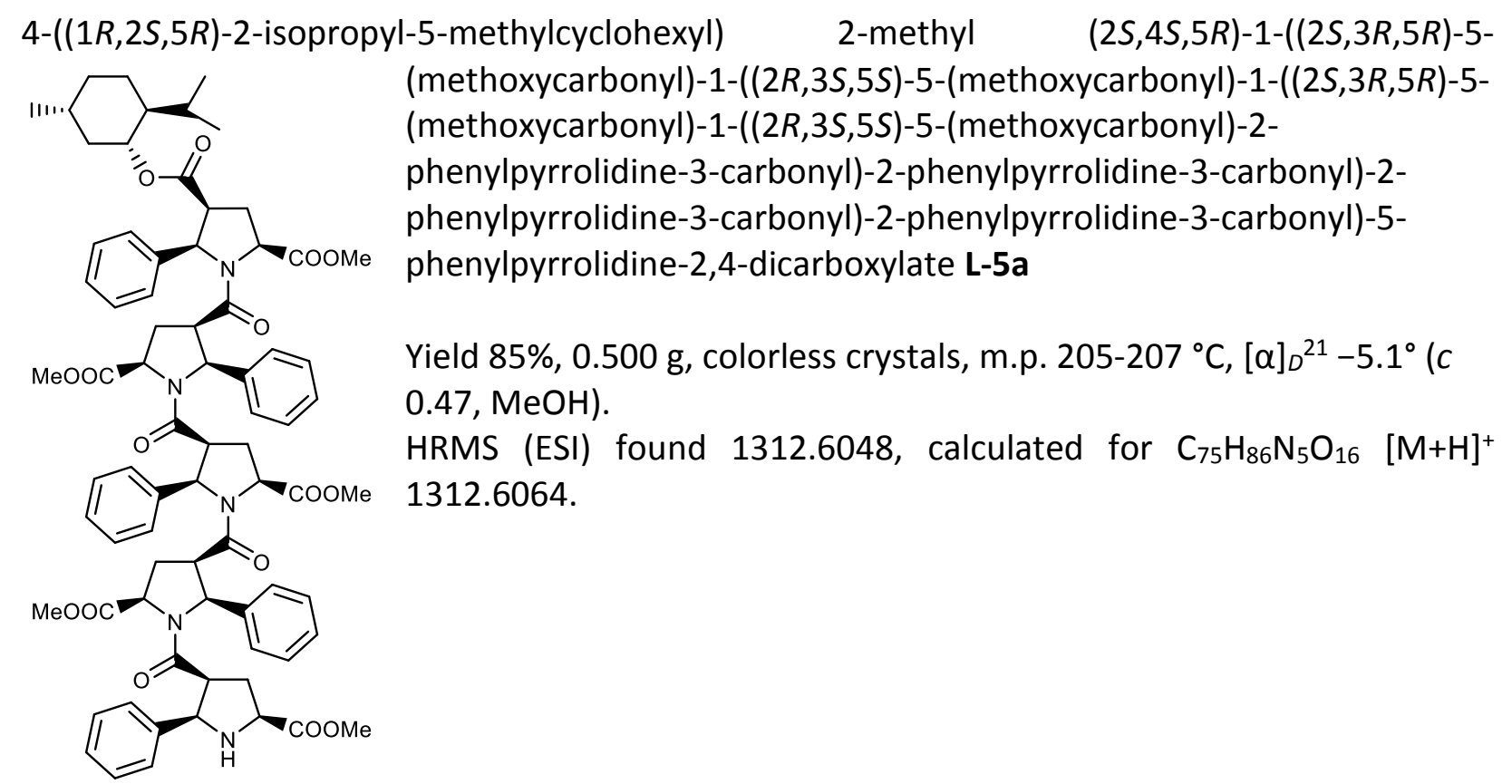

4-((1R,2S,5R)-2-isopropyl-5-methylcyclohexyl) 2-methyl $\quad(2 S, 4 S, 5 R)-5$-(4-bromophenyl)-1-

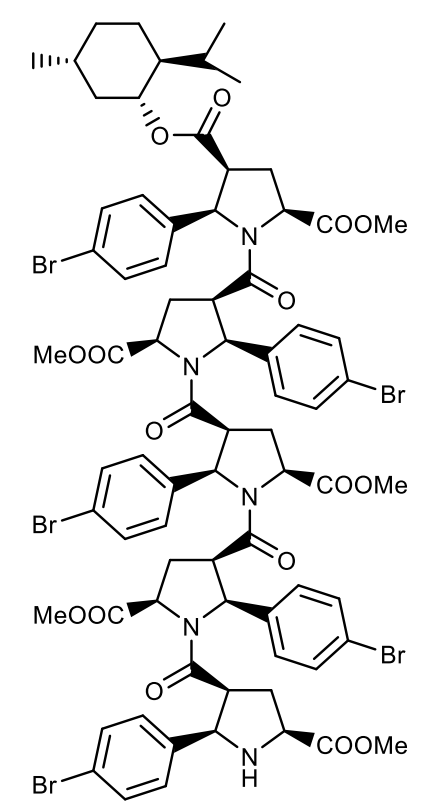

((2S,3R,5R)-2-(4-bromophenyl)-1-((2R,3S,5S)-2-(4-bromophenyl)-1-

((2S,3R,5R)-2-(4-bromophenyl)-1-((2R,3S,5S)-2-(4-bromophenyl)-5-

(methoxycarbonyl)pyrrolidine-3-carbonyl)-5-

(methoxycarbonyl)pyrrolidine-3-carbonyl)-5-

(methoxycarbonyl)pyrrolidine-3-carbonyl)-5-

(methoxycarbonyl)pyrrolidine-3-carbonyl)pyrrolidine-2,4-

dicarboxylate $\mathbf{L}-\mathbf{5 b}$

Yield $78 \%, 0.900 \mathrm{~g}$, colorless crystals, m.p. $216-218^{\circ} \mathrm{C},[\alpha]_{D}{ }^{21}-13.1^{\circ}(\mathrm{c}$ $0.29, \mathrm{CH}_{2} \mathrm{Cl}_{2}$ ).

HRMS (ESI) calculated for $\mathrm{C}_{75} \mathrm{H}_{81} \mathrm{Br}_{5} \mathrm{~N}_{5} \mathrm{O}_{16}[\mathrm{M}+\mathrm{H}]^{+} 1702.1563$, found 1702.1625 .

2-(tert-butyl) $\quad 4-((1 R, 2 S, 5 R)$-2-isopropyl-5-methylcyclohexyl) $\quad(2 S, 4 S, 5 R)-1-((2 S, 3 R, 5 R)-5$-(tert-

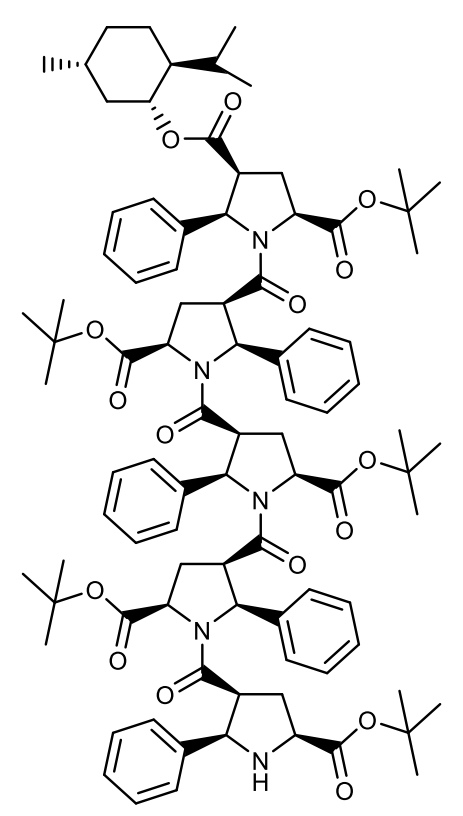
butoxycarbonyl)-1-((2R,3S,5S)-5-(tert-butoxycarbonyl)-1$((2 S, 3 R, 5 R)-5$-(tert-butoxycarbonyl)-1- $((2 R, 3 S, 5 S)-5$-(tertbutoxycarbonyl)-2-phenylpyrrolidine-3-carbonyl)-2phenylpyrrolidine-3-carbonyl)-2-phenylpyrrolidine-3-carbonyl)-2phenylpyrrolidine-3-carbonyl)-5-phenylpyrrolidine-2,4dicarboxylate L-5C

Yield $81 \%, 3.000$ g, colorless crystals, m.p. $176-178^{\circ} \mathrm{C},[\alpha]_{D}{ }^{21}-1.55^{\circ}$ (c $0.75, \mathrm{MeOH})$. 
HRMS (ESI) calculated for $\mathrm{C}_{90} \mathrm{H}_{116} \mathrm{~N}_{5} \mathrm{O}_{16}[\mathrm{M}+\mathrm{H}]^{+} 1522.8412$, found 1522.8465 .

4-((1S,2R,5S)-2-isopropyl-5-methylcyclohexyl) 2-methyl $\quad(2 R, 4 R, 5 S)-1-((2 R, 3 S, 5 S)-5-$

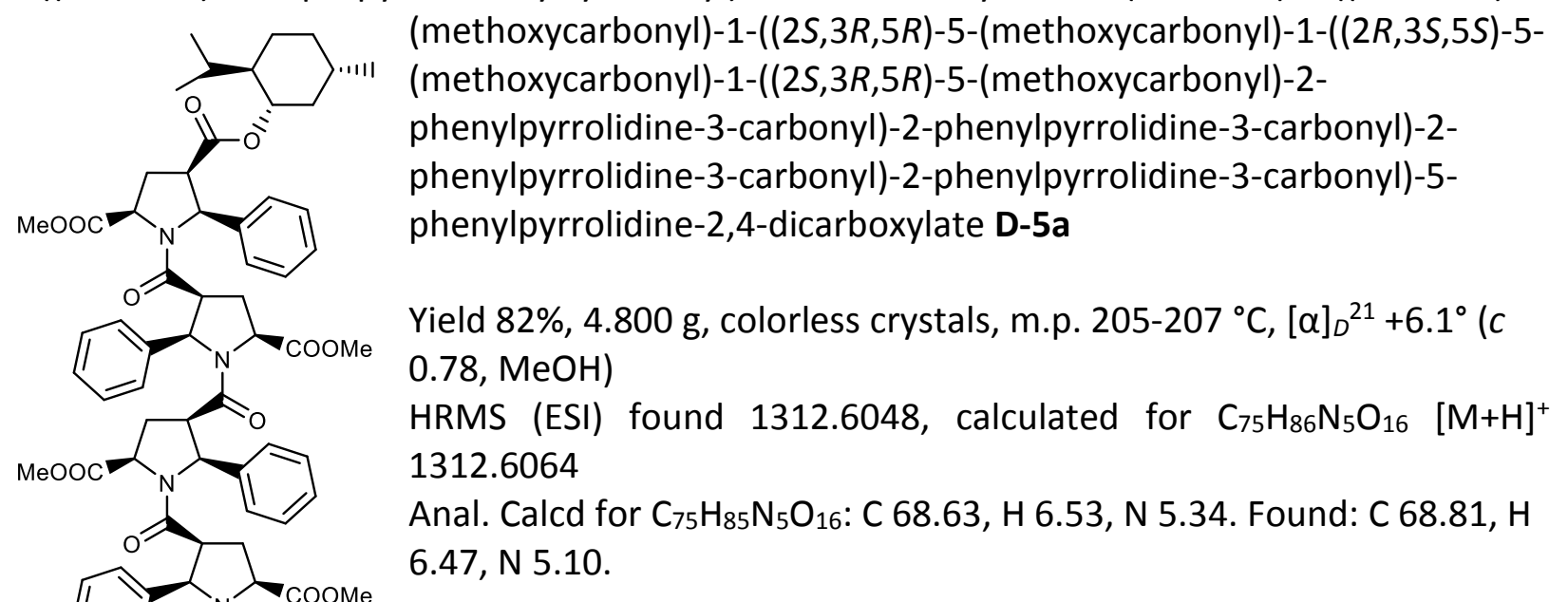

4-((1R,2S,5R)-2-isopropyl-5-methylcyclohexyl) 2-methyl $(2 S, 4 S, 5 R)-1-((2 S, 3 R, 5 R)-1-((2 R, 3 S, 5 S)$ -

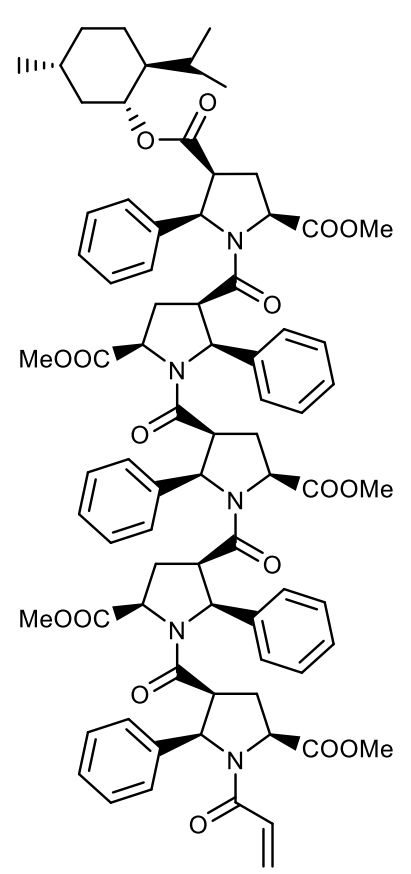
1-((2S,3R,5R)-1-((2R,3S,5S)-1-acryloyl-5-(methoxycarbonyl)-2phenylpyrrolidine-3-carbonyl)-5-(methoxycarbonyl)-2phenylpyrrolidine-3-carbonyl)-5-(methoxycarbonyl)-2phenylpyrrolidine-3-carbonyl)-5-(methoxycarbonyl)-2phenylpyrrolidine-3-carbonyl)-5-phenylpyrrolidine-2,4dicarboxylate L-5a_A

Yield $76 \%, 0.300 \mathrm{~g}$, colorless crystals, m.p. $221-223^{\circ} \mathrm{C},[\alpha]_{D^{21}}-36.0^{\circ}$ (c $0.25, \mathrm{MeOH}$ ).

HRMS (ESI) calculated for $\mathrm{C}_{78} \mathrm{H}_{87} \mathrm{~N}_{5} \mathrm{NaO}_{17}[\mathrm{M}+\mathrm{Na}]^{+}$1388.5994, found 1388.5999. 
4-((1R,2S,5R)-2-isopropyl-5-methylcyclohexyl) 2-methyl $\quad(2 S, 4 S, 5 R)-1-((2 S, 3 R, 5 R)-1-((2 R, 3 S, 5 S)$ -

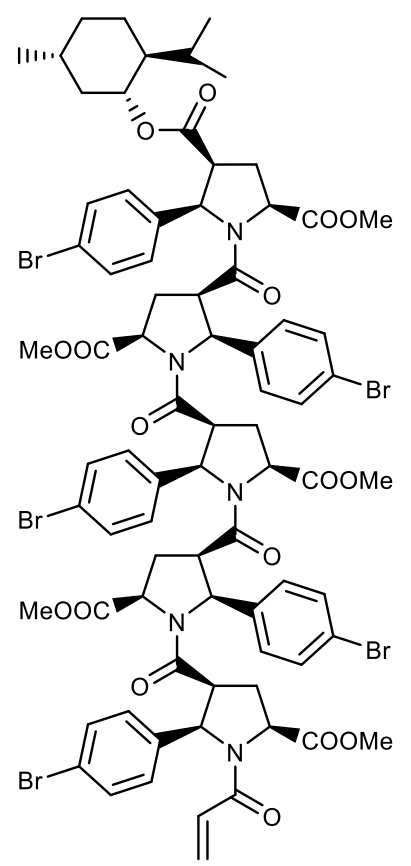
1-((2S,3R,5R)-1-((2R,3S,5S)-1-acryloyl-2-(4-bromophenyl)-5(methoxycarbonyl)pyrrolidine-3-carbonyl)-2-(4-bromophenyl)-5(methoxycarbonyl)pyrrolidine-3-carbonyl)-2-(4-bromophenyl)-5(methoxycarbonyl)pyrrolidine-3-carbonyl)-2-(4-bromophenyl)-5(methoxycarbonyl)pyrrolidine-3-carbonyl)-5-(4bromophenyl)pyrrolidine-2,4-dicarboxylate L-5b_A

Yield $78 \%, 0.510$ g, colorless crystals, m.p. $230-232{ }^{\circ} \mathrm{C},[\alpha]_{D}{ }^{21}-29.6^{\circ}$ (c $0.27, \mathrm{CH}_{2} \mathrm{Cl}_{2}$ ).

HRMS (ESI) calculated for $\mathrm{C}_{78} \mathrm{H}_{83} \mathrm{~N}_{5} \mathrm{Br}_{5} \mathrm{O}_{17}[\mathrm{M}+\mathrm{H}]^{+} 1756.1700$, found 1756.1702 .

\footnotetext{
2-(tert-butyl) $\quad$ 4-((1R,2S,5R)-2-isopropyl-5-methylcyclohexyl) $\quad(2 S, 4 S, 5 R)-1-((2 S, 3 R, 5 R)-1$ -

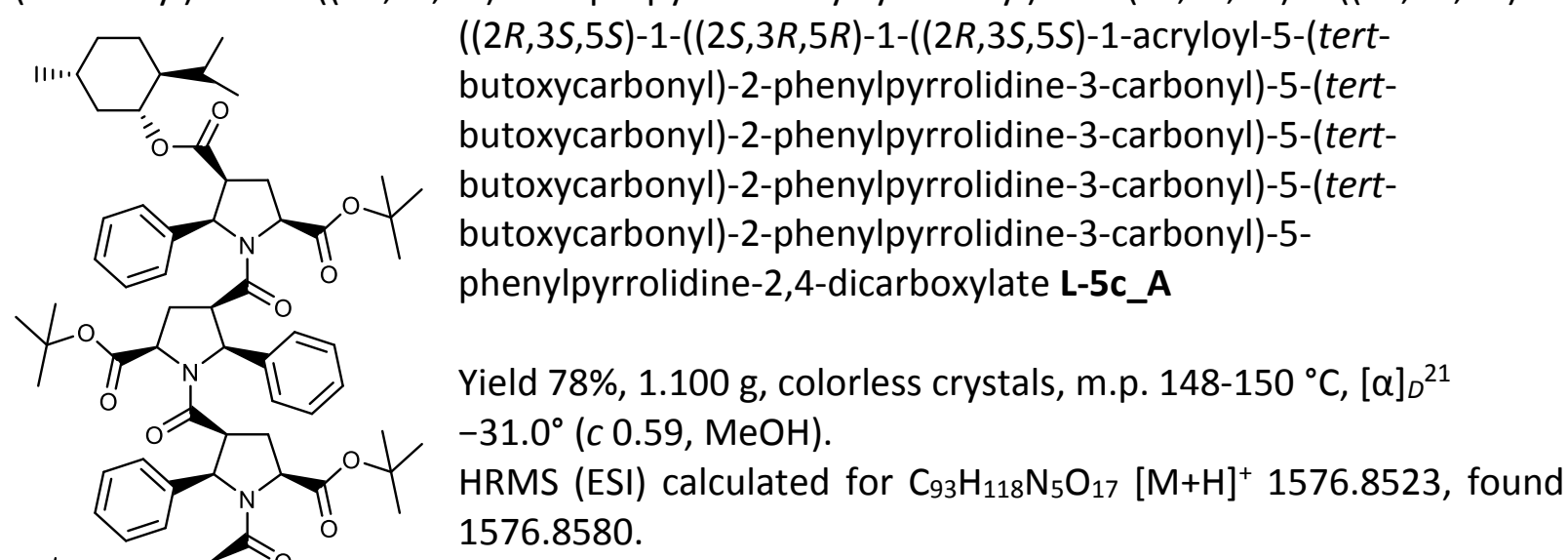


4-((1S,2R,5S)-2-isopropyl-5-methylcyclohexyl) 2-methyl $\quad(2 R, 4 R, 5 S)-1-((2 R, 3 S, 5 S)-1-((2 S, 3 R, 5 R)-$

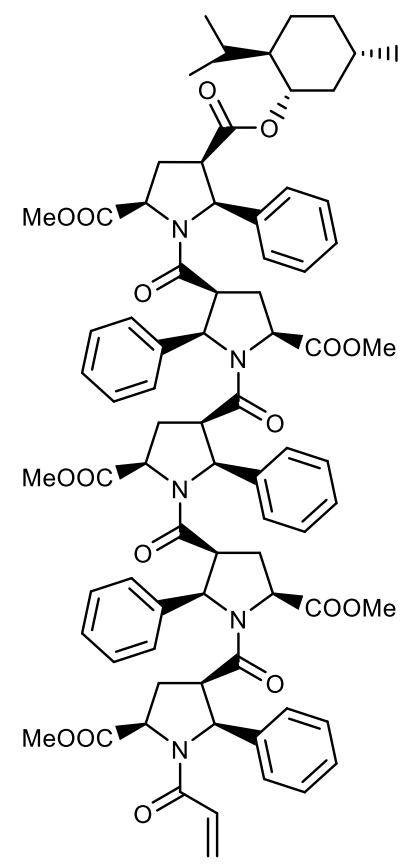
1-((2R,3S,5S)-1-((2S,3R,5R)-1-acryloyl-5-(methoxycarbonyl)-2phenylpyrrolidine-3-carbonyl)-5-(methoxycarbonyl)-2phenylpyrrolidine-3-carbonyl)-5-(methoxycarbonyl)-2phenylpyrrolidine-3-carbonyl)-5-(methoxycarbonyl)-2phenylpyrrolidine-3-carbonyl)-5-phenylpyrrolidine-2,4-dicarboxylate D-5a_A

Yield $80 \%, 3.700$ g, colorless crystals, m.p. $221-223^{\circ} \mathrm{C},[\alpha]_{D}^{21}+38.6^{\circ}(\mathrm{c}$ $0.49, \mathrm{MeOH})$.

HRMS (ESI) calculated for $\mathrm{C}_{78} \mathrm{H}_{87} \mathrm{~N}_{5} \mathrm{NaO}_{17}[\mathrm{M}+\mathrm{Na}]^{+} 1388.5994$, found 1388.5999.

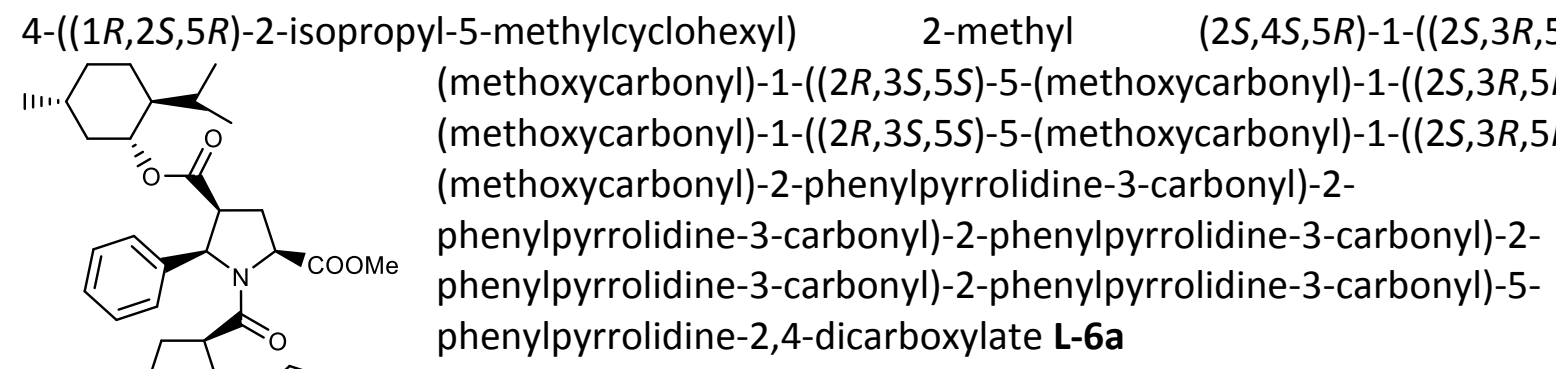

Yield $75 \%, 0.190$ g, colorless crystals, m.p. $208-210^{\circ} \mathrm{C}$, $[\alpha]_{D}{ }^{21}-15.0^{\circ}$ (c $0.86, \mathrm{MeOH})$.

HRMS (ESI) calculated for $\mathrm{C}_{88} \mathrm{H}_{99} \mathrm{~N}_{6} \mathrm{O}_{19}[\mathrm{M}+\mathrm{H}]^{+}$1543.6960, found 1543.7035 
4-((1R,2S,5R)-2-isopropyl-5-methylcyclohexyl) 2-methyl $\quad(2 S, 4 S, 5 R)-5$-(4-bromophenyl)-1-

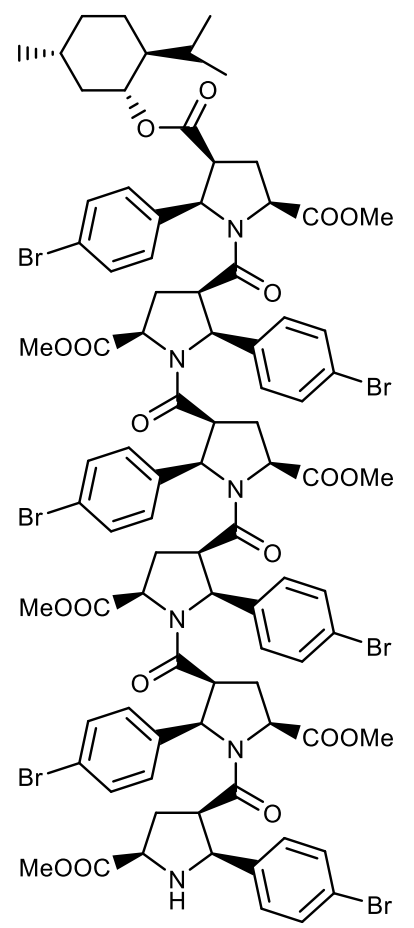
((2S,3R,5R)-2-(4-bromophenyl)-1-((2R,3S,5S)-2-(4-bromophenyl)-1((2S,3R,5R)-2-(4-bromophenyl)-1-((2R,3S,5S)-2-(4-bromophenyl)-1((2S,3R,5R)-2-(4-bromophenyl)-5-(methoxycarbonyl)pyrrolidine-3carbonyl)-5-(methoxycarbonyl)pyrrolidine-3-carbonyl)-5-

(methoxycarbonyl)pyrrolidine-3-carbonyl)-5(methoxycarbonyl)pyrrolidine-3-carbonyl)-5(methoxycarbonyl)pyrrolidine-3-carbonyl)pyrrolidine-2,4dicarboxylate L-6b

Yield $71 \%, 0.530 \mathrm{~g}$, colorless crystals, m.p. $224-226^{\circ} \mathrm{C},[\alpha]_{D}^{21}+35.6^{\circ}(\mathrm{c}$ $\left.0.25, \mathrm{CH}_{2} \mathrm{Cl}_{2}\right)$.

HRMS (ESI), calculated for $\mathrm{C}_{88} \mathrm{H}_{93} \mathrm{Br}_{6} \mathrm{~N}_{6} \mathrm{O}_{19}[\mathrm{M}+\mathrm{H}]^{+} 2011.1596$, found 2011.1590.

2-(tert-butyl) 4-((1R,2S,5R)-2-isopropyl-5-methylcyclohexyl) $(2 S, 4 S, 5 R)-1-((2 S, 3 R, 5 R)-5$-(tert-

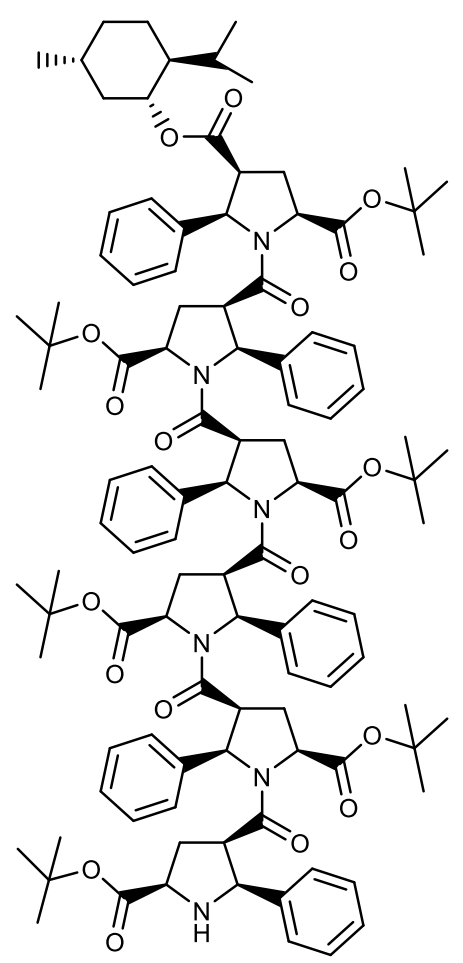
butoxycarbonyl)-1-((2R,3S,5S)-5-(tert-butoxycarbonyl)-1$((2 S, 3 R, 5 R)-5$-(tert-butoxycarbonyl)-1-((2R,3S,5S)-5-(tertbutoxycarbonyl)-1-((2S,3R,5R)-5-(tert-butoxycarbonyl)-2phenylpyrrolidine-3-carbonyl)-2-phenylpyrrolidine-3-carbonyl)-2phenylpyrrolidine-3-carbonyl)-2-phenylpyrrolidine-3-carbonyl)-2phenylpyrrolidine-3-carbonyl)-5-phenylpyrrolidine-2,4dicarboxylate L-6c

Yield $83 \%, 0.640 \mathrm{~g}$, colorless crystals, m.p. $188-190{ }^{\circ} \mathrm{C},[\alpha]_{D^{21}}-19.7^{\circ}$ (c $0.39, \mathrm{MeOH}$ ).

HRMS (ESI) calculated for $\mathrm{C}_{106} \mathrm{H}_{135} \mathrm{~N}_{6} \mathrm{O}_{19}[\mathrm{M}+\mathrm{H}]^{+} 1795.9782$, found 1795.9785. 


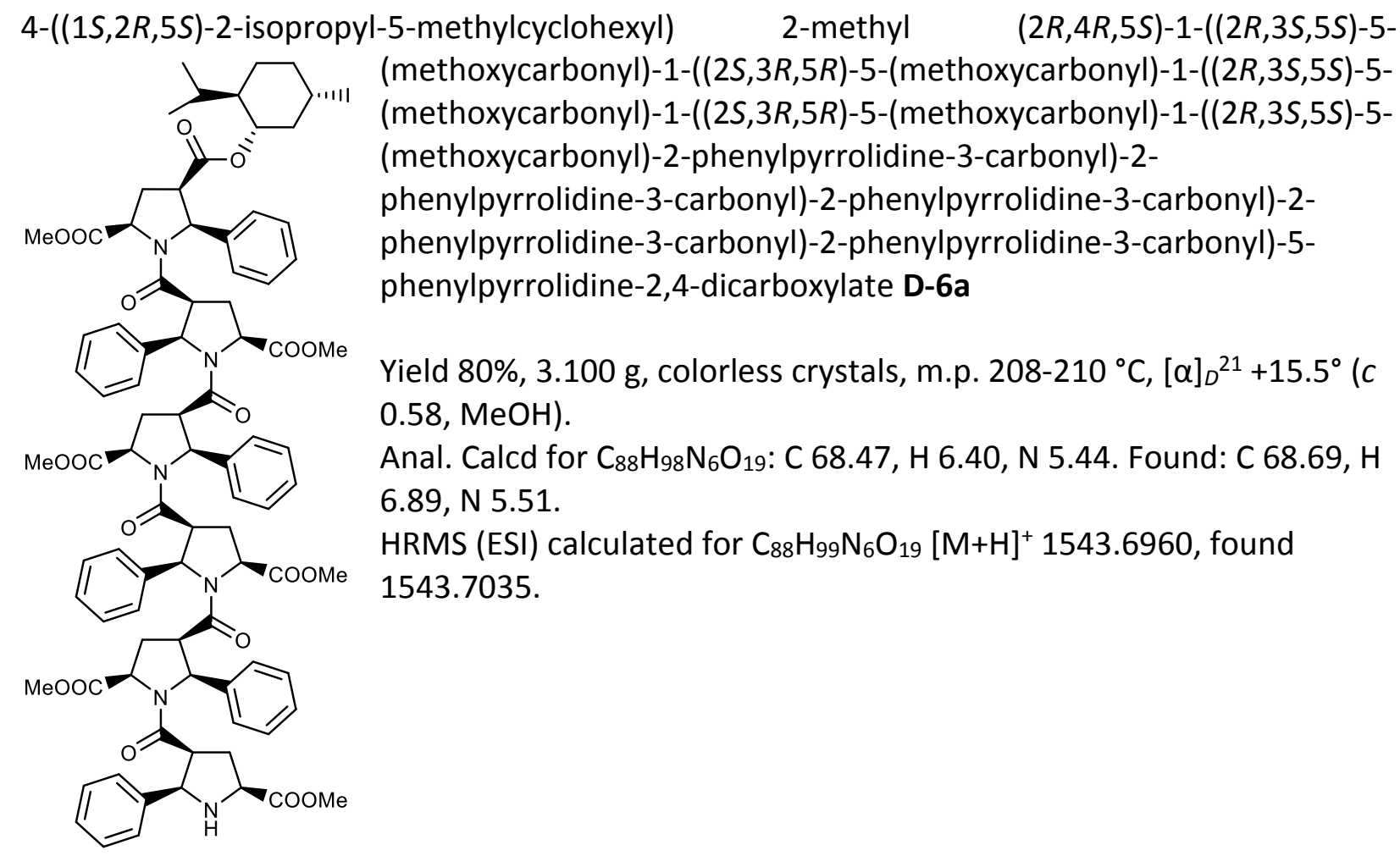



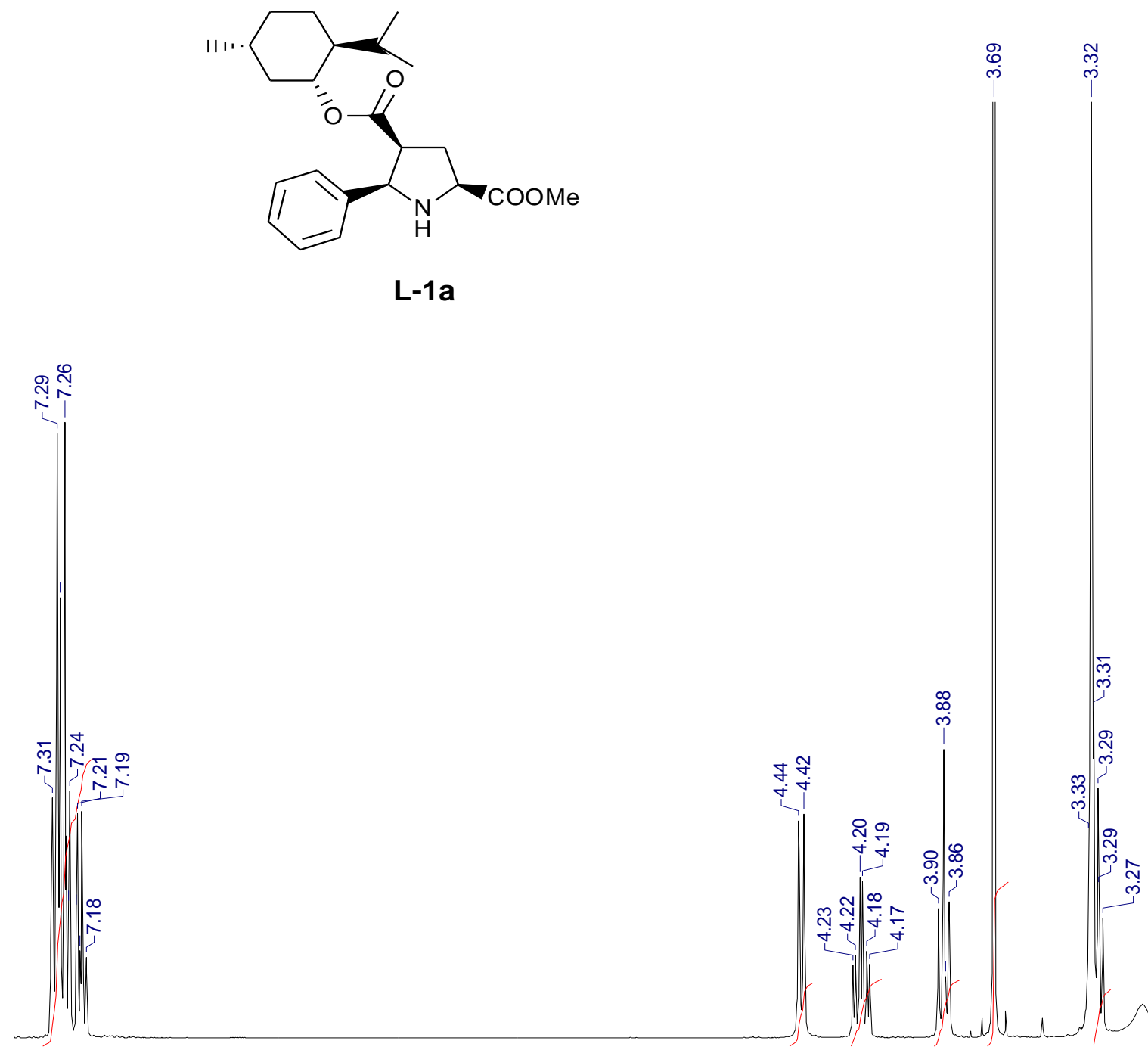

DMSO-d6
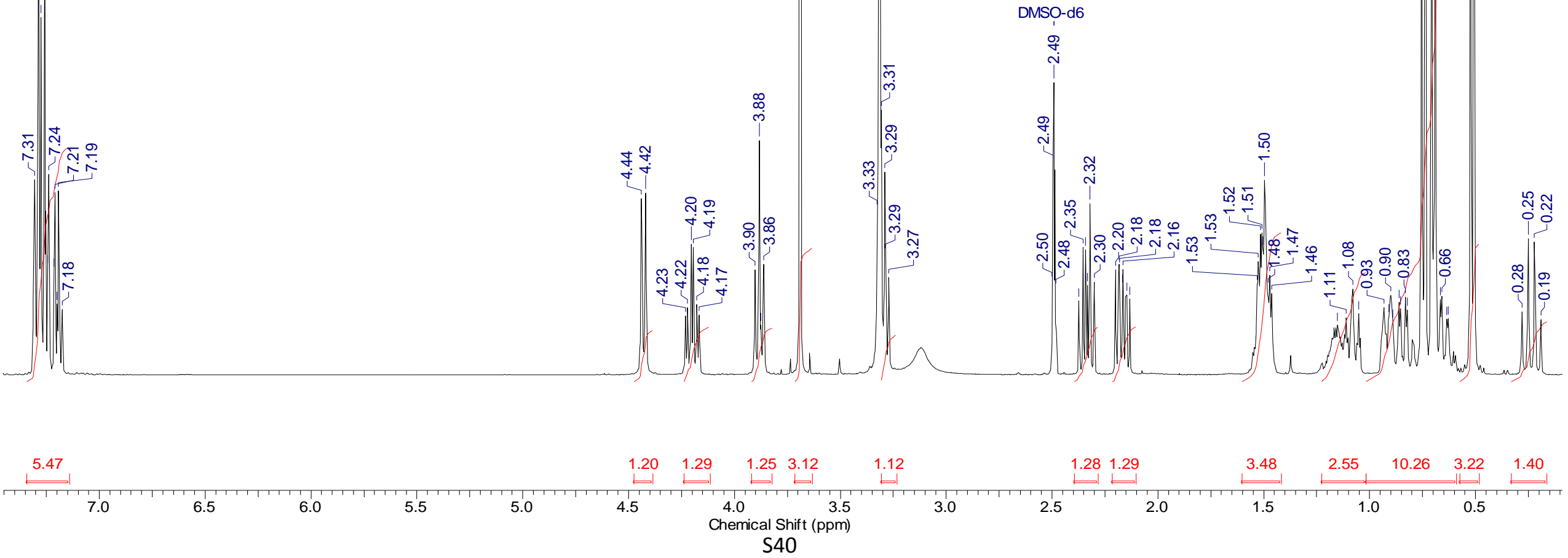


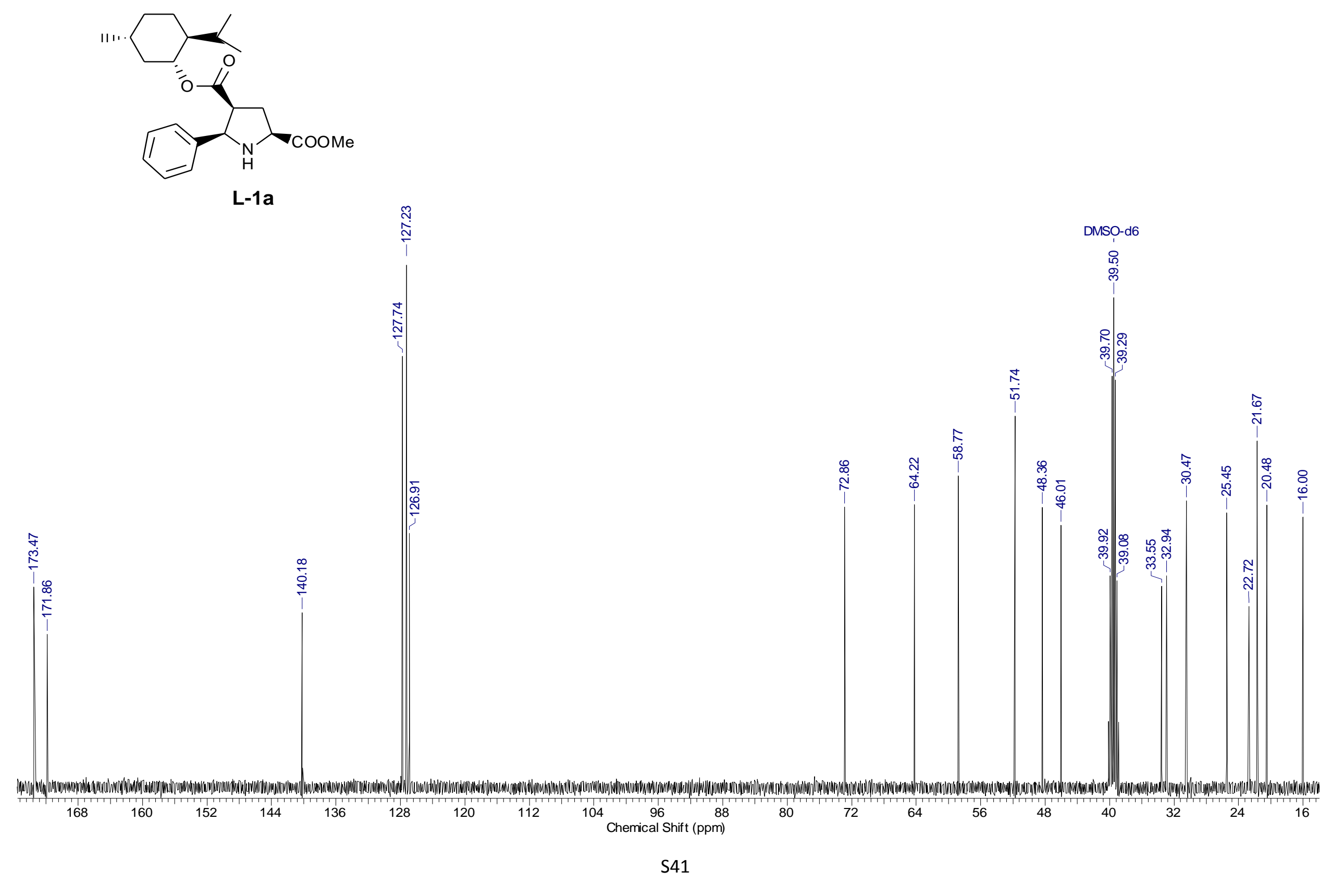



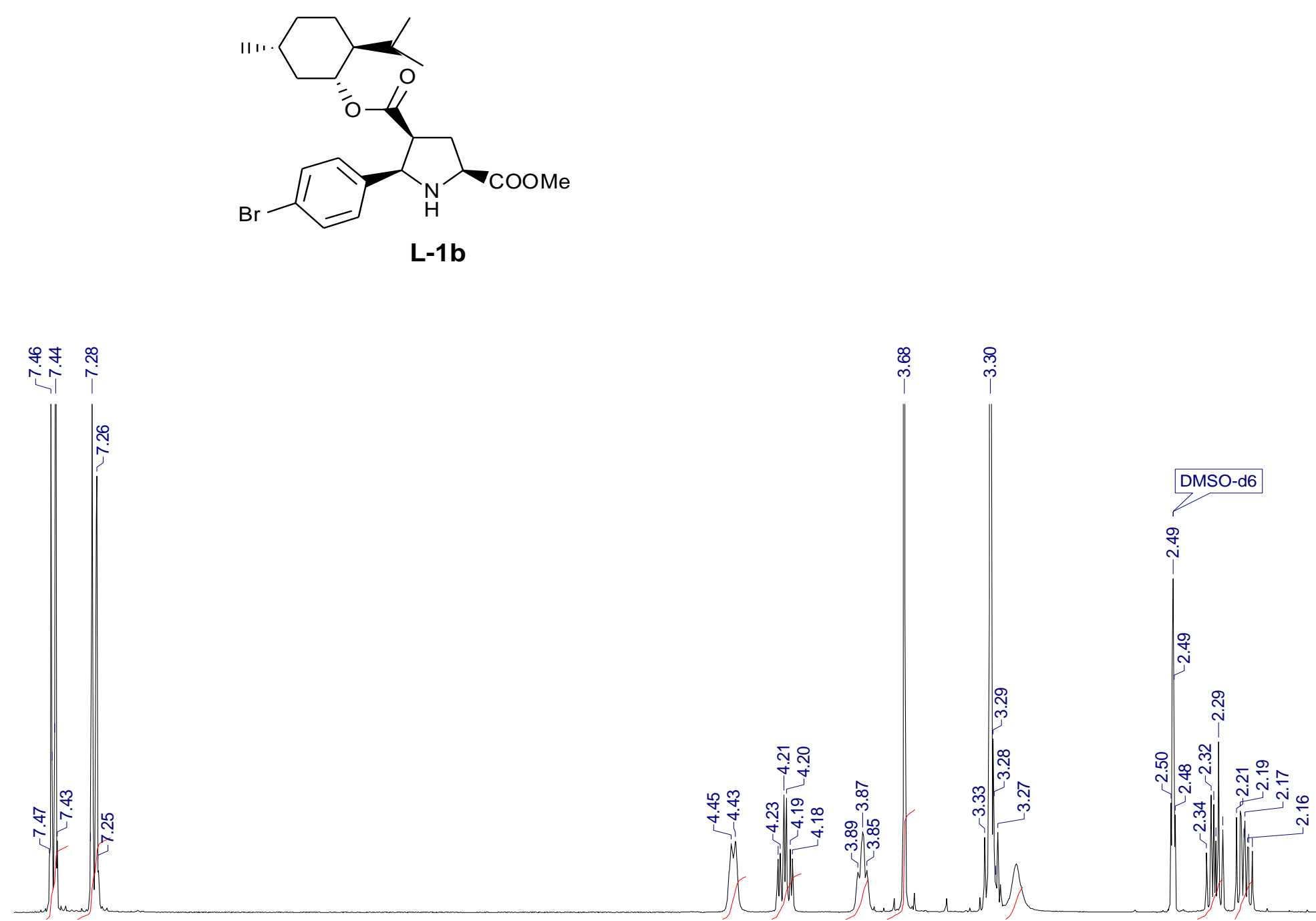

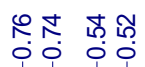

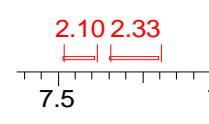



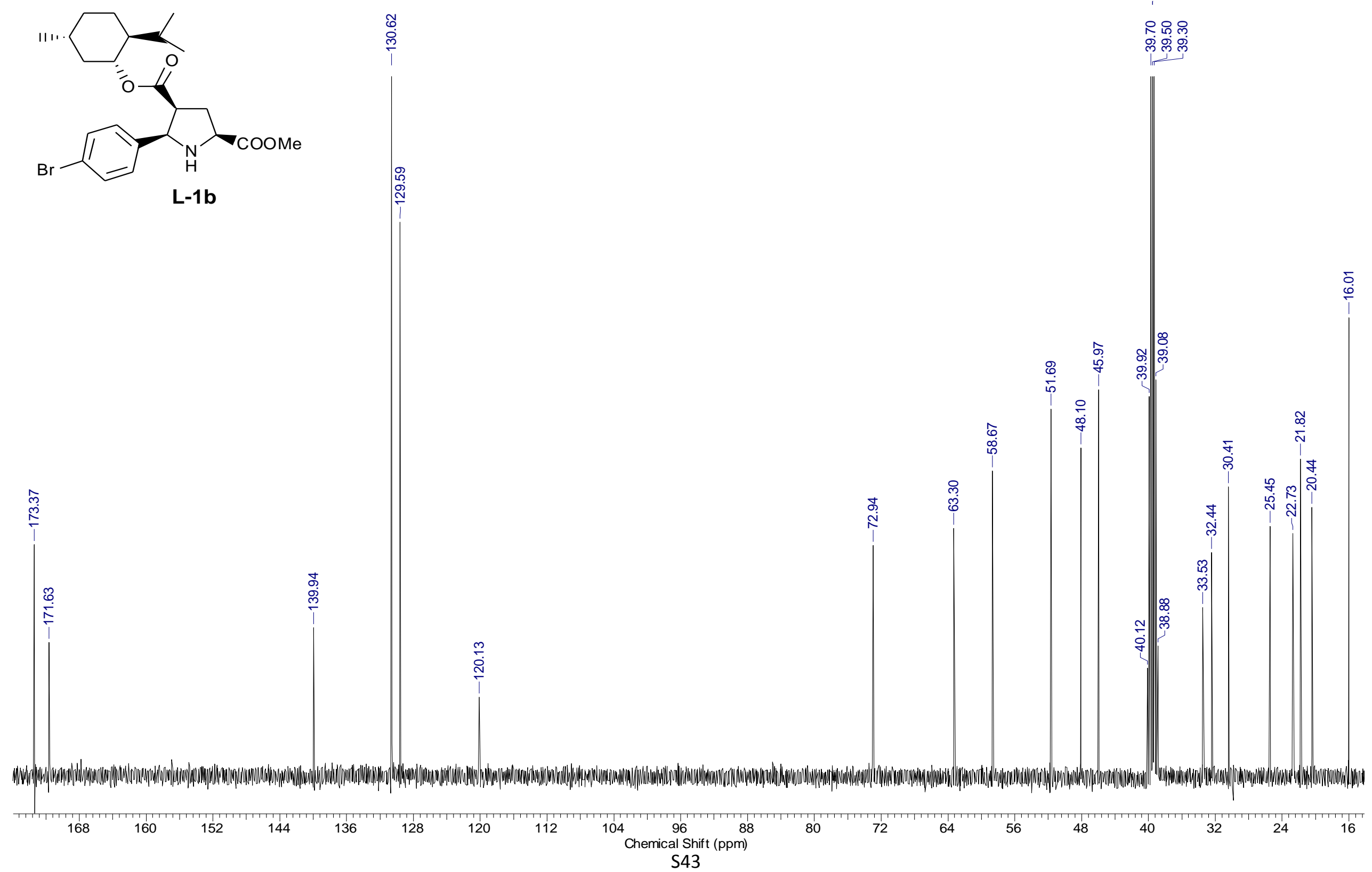

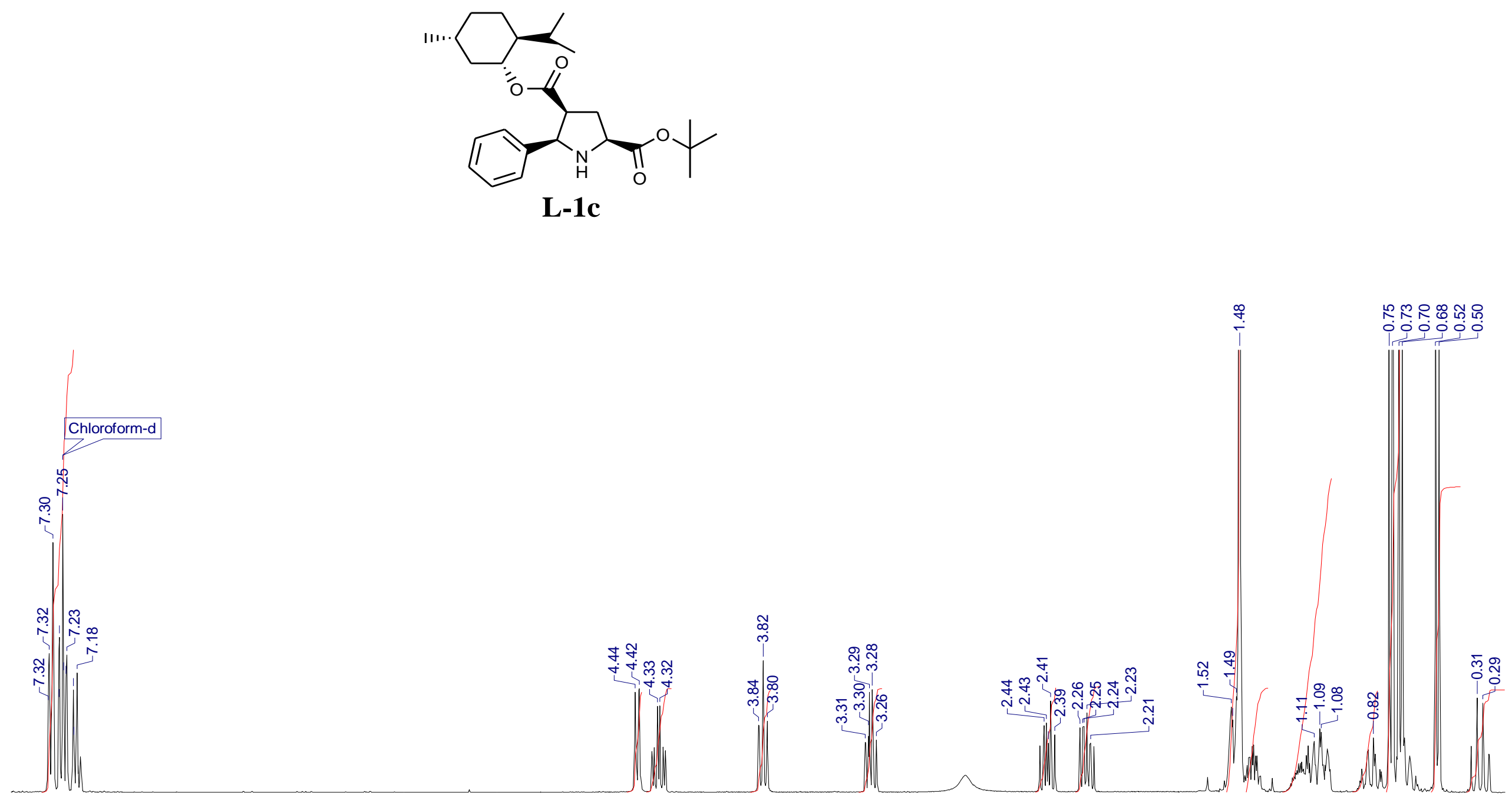

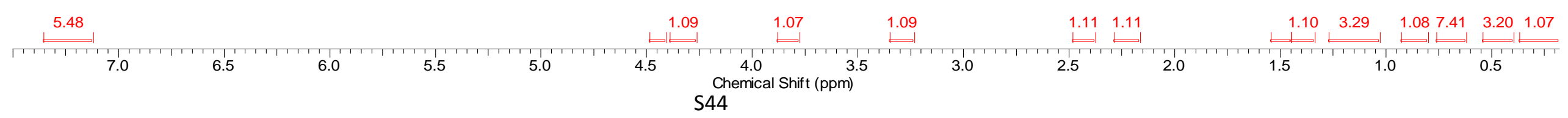



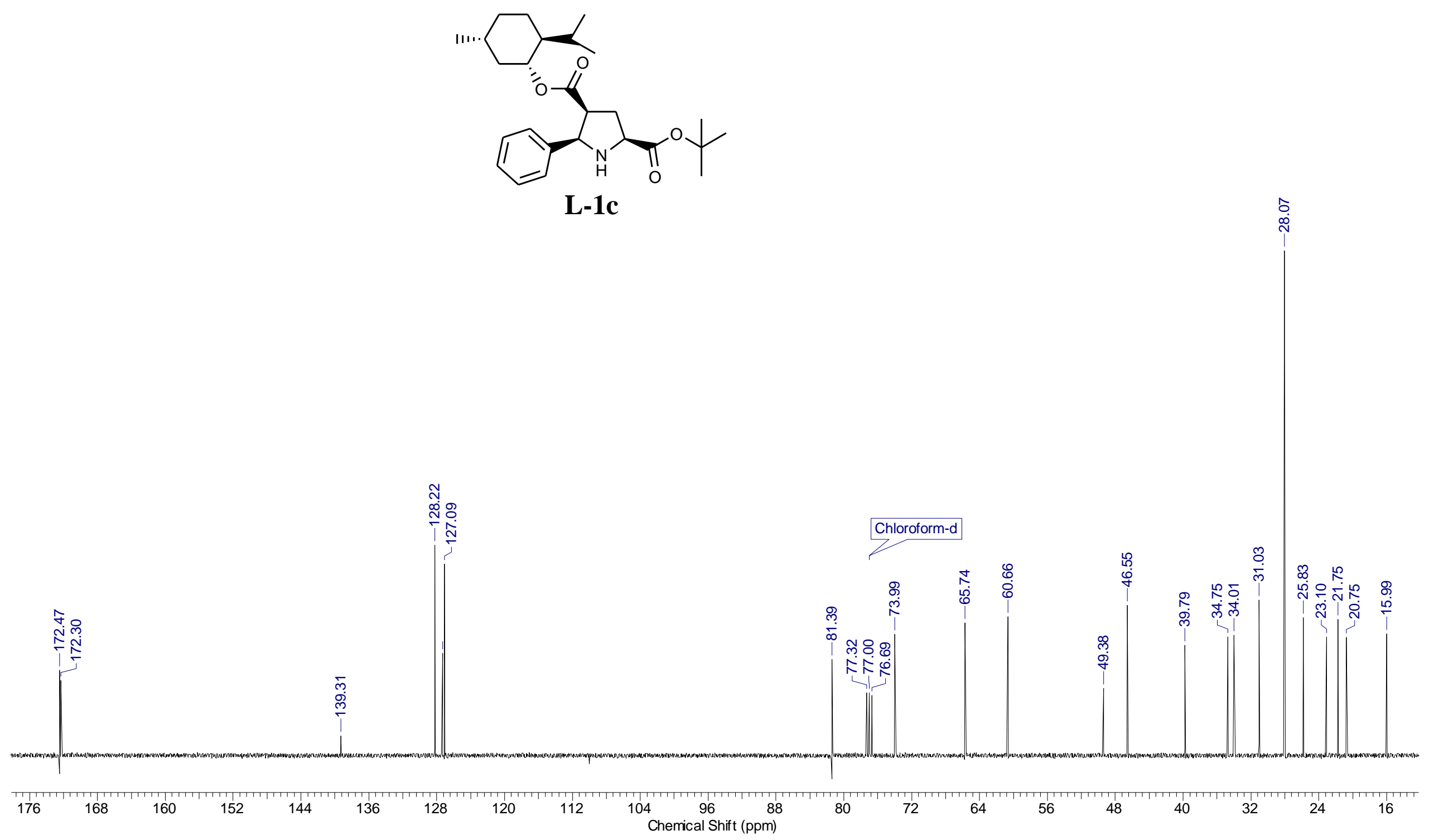


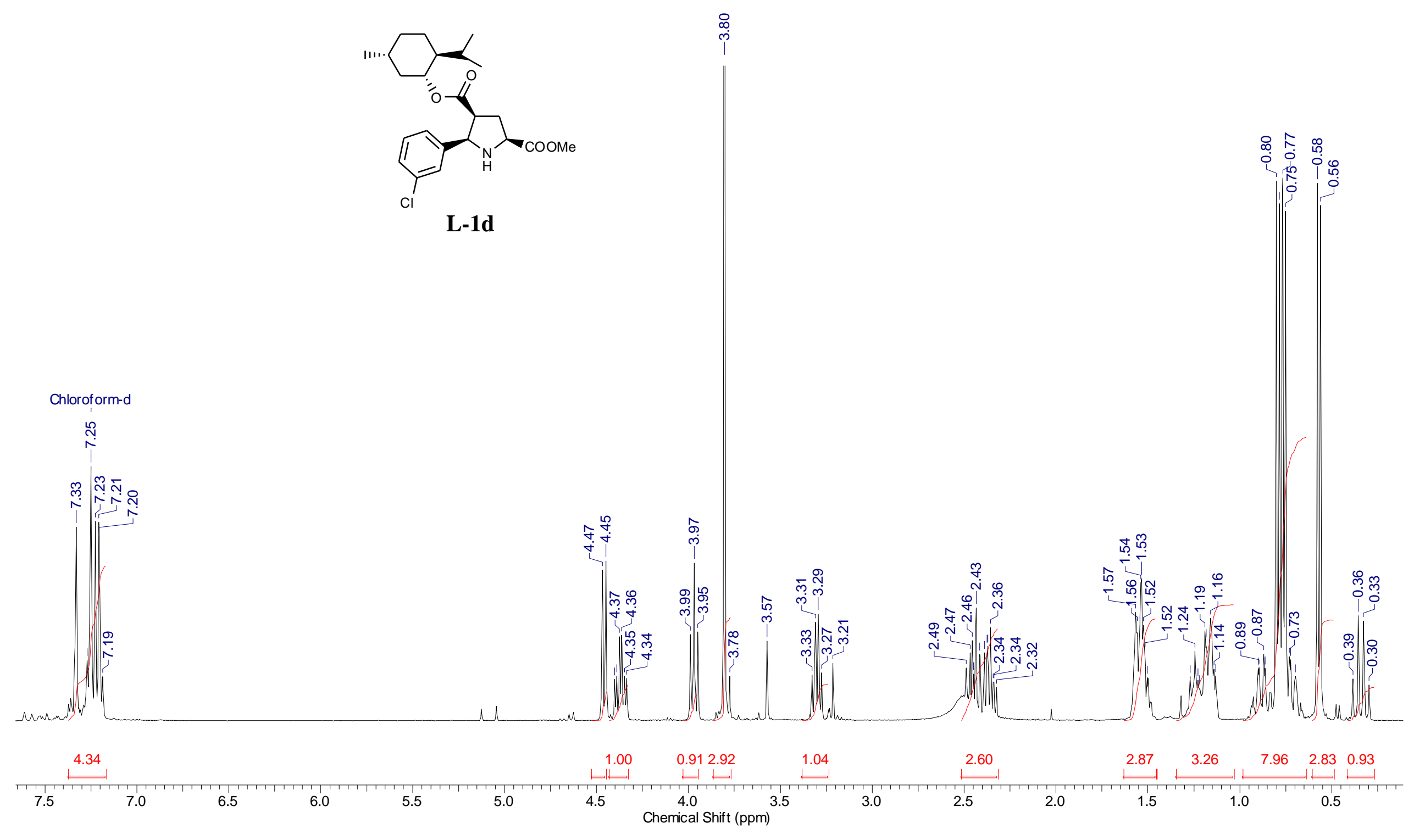



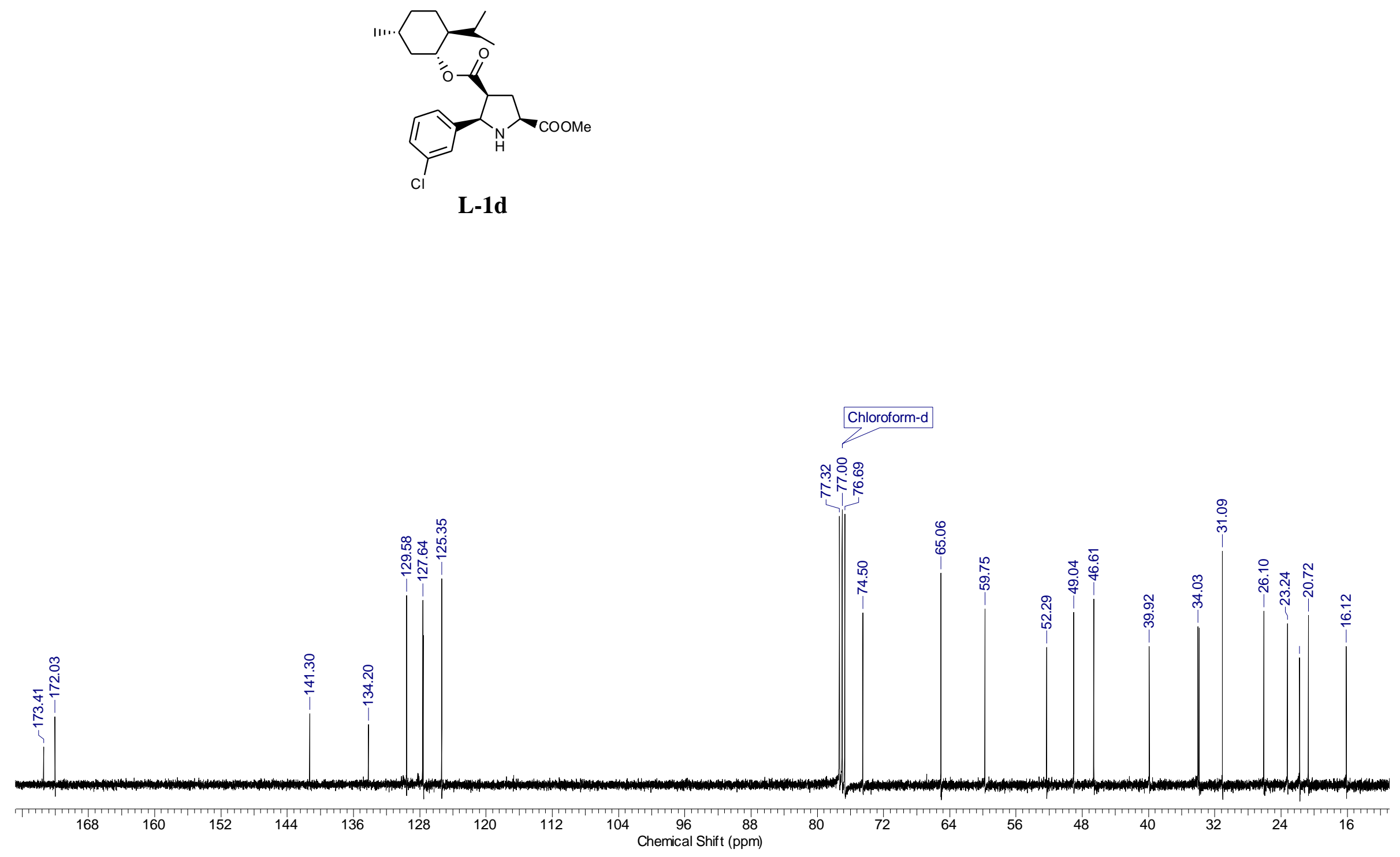


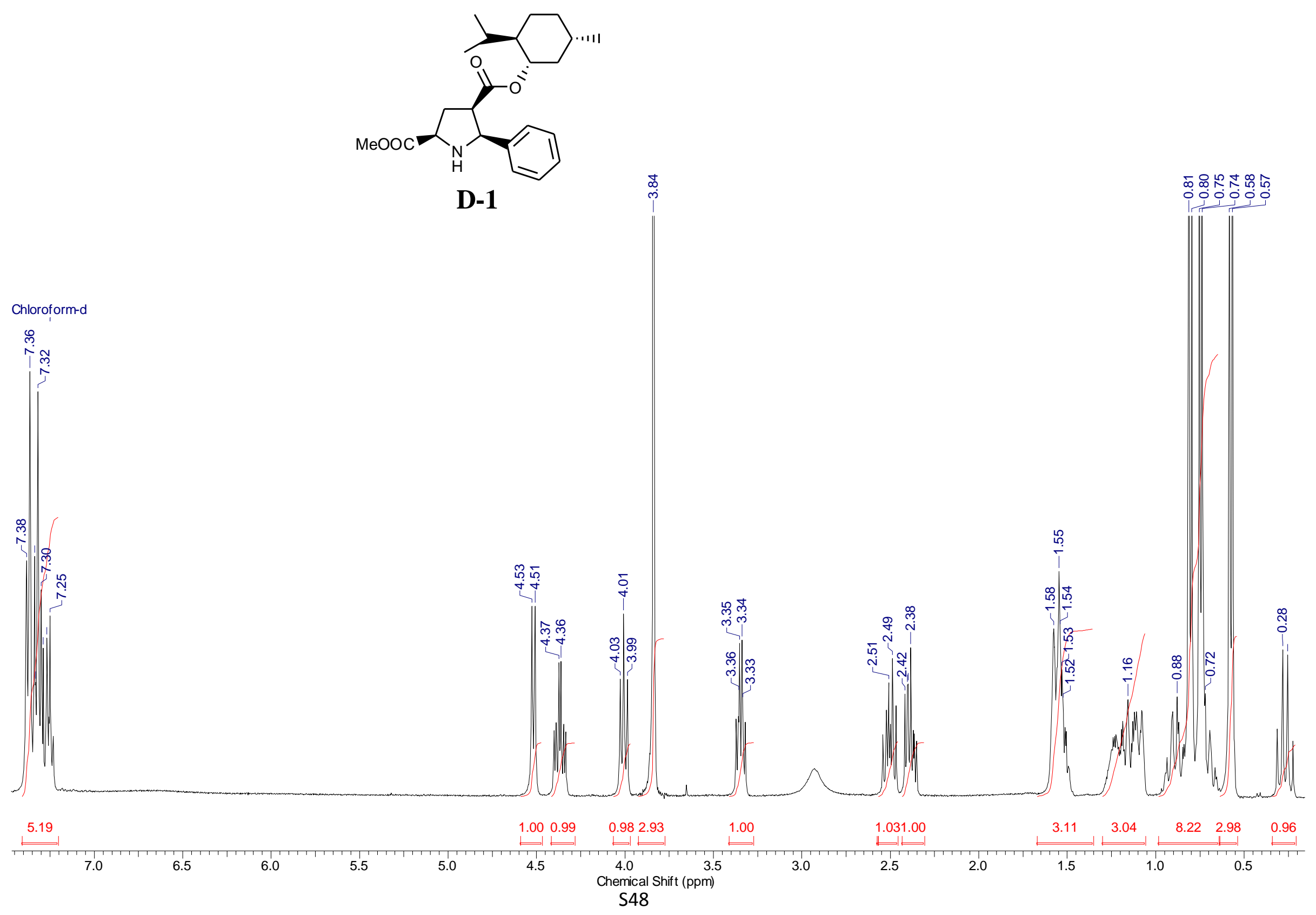



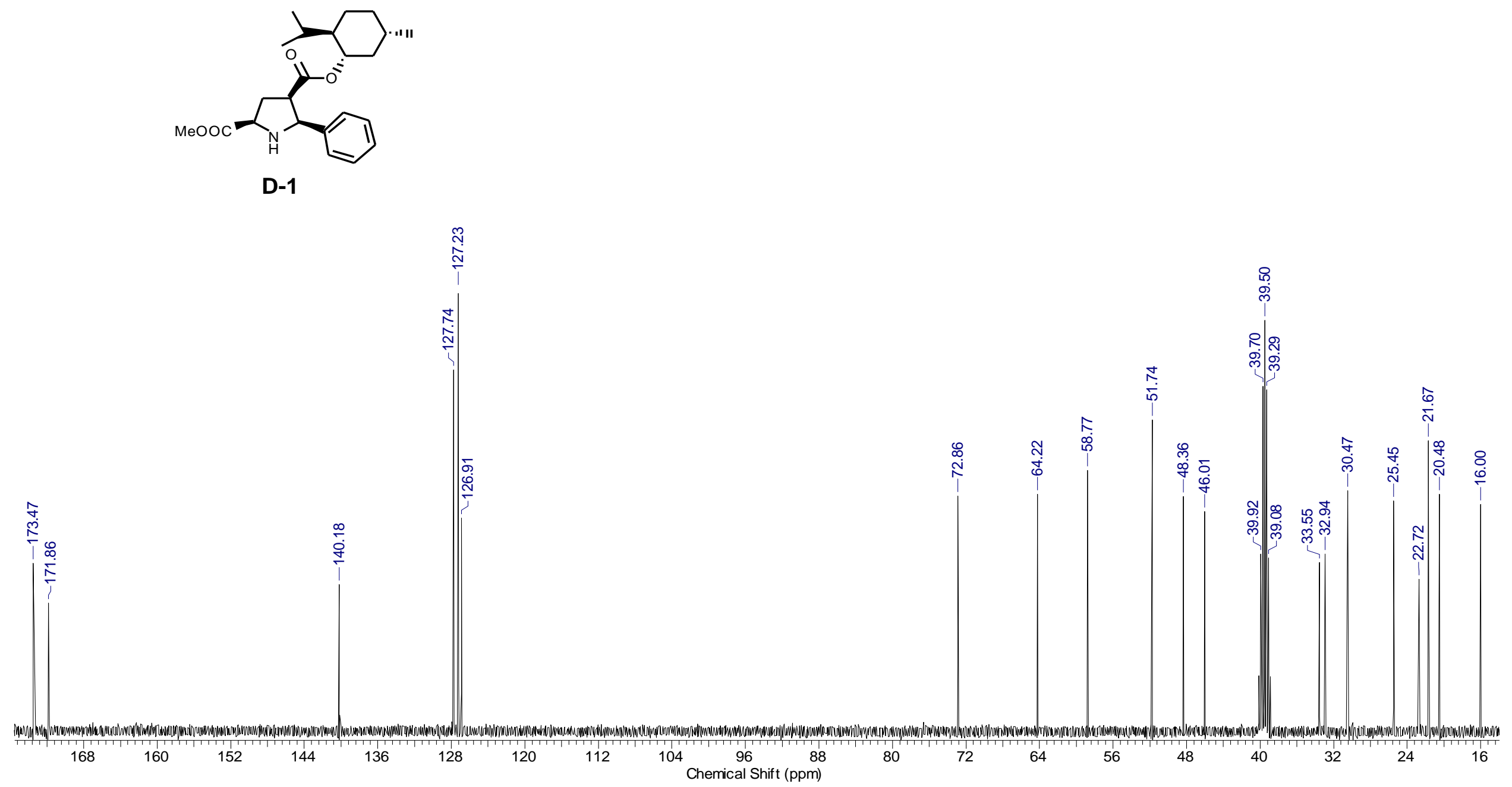


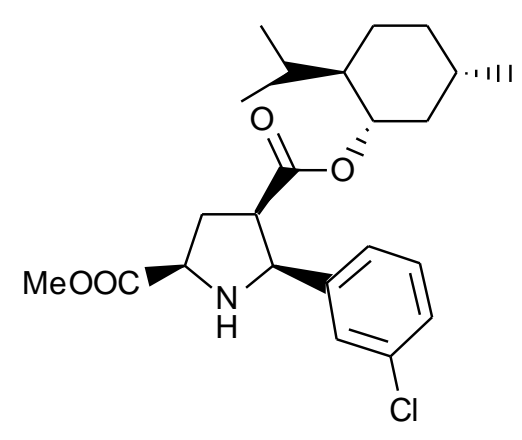

D-1d

Chloroform-d
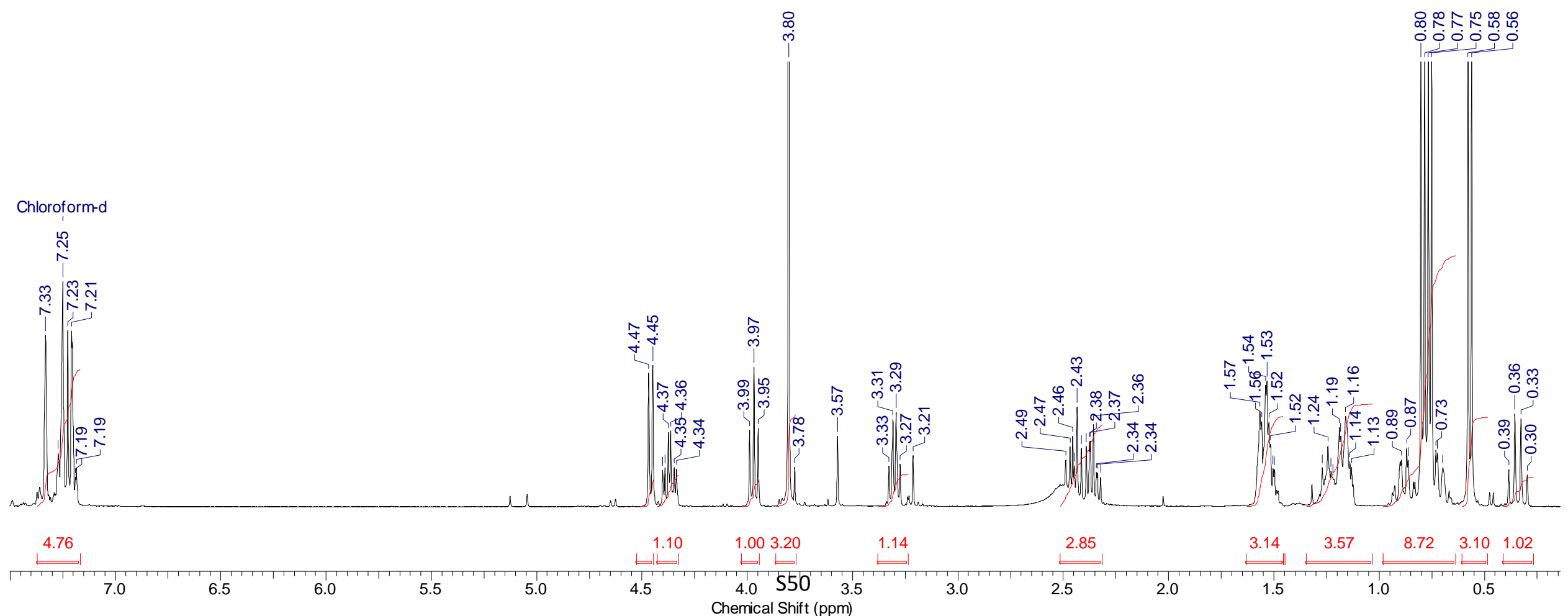


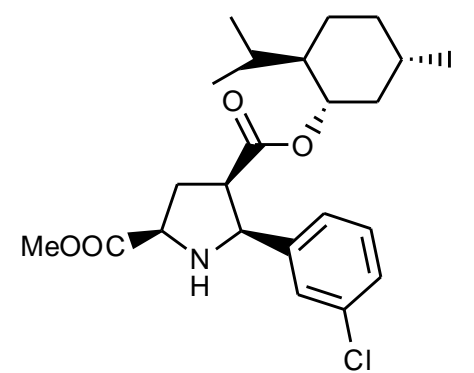

D-1d

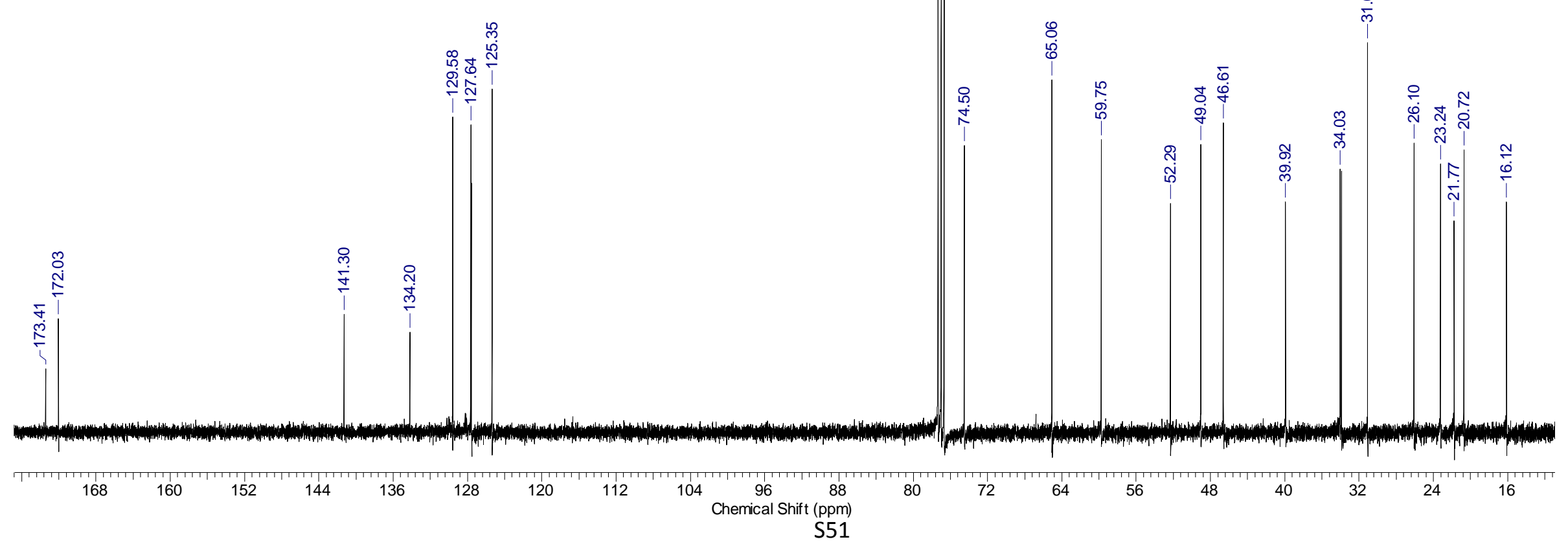



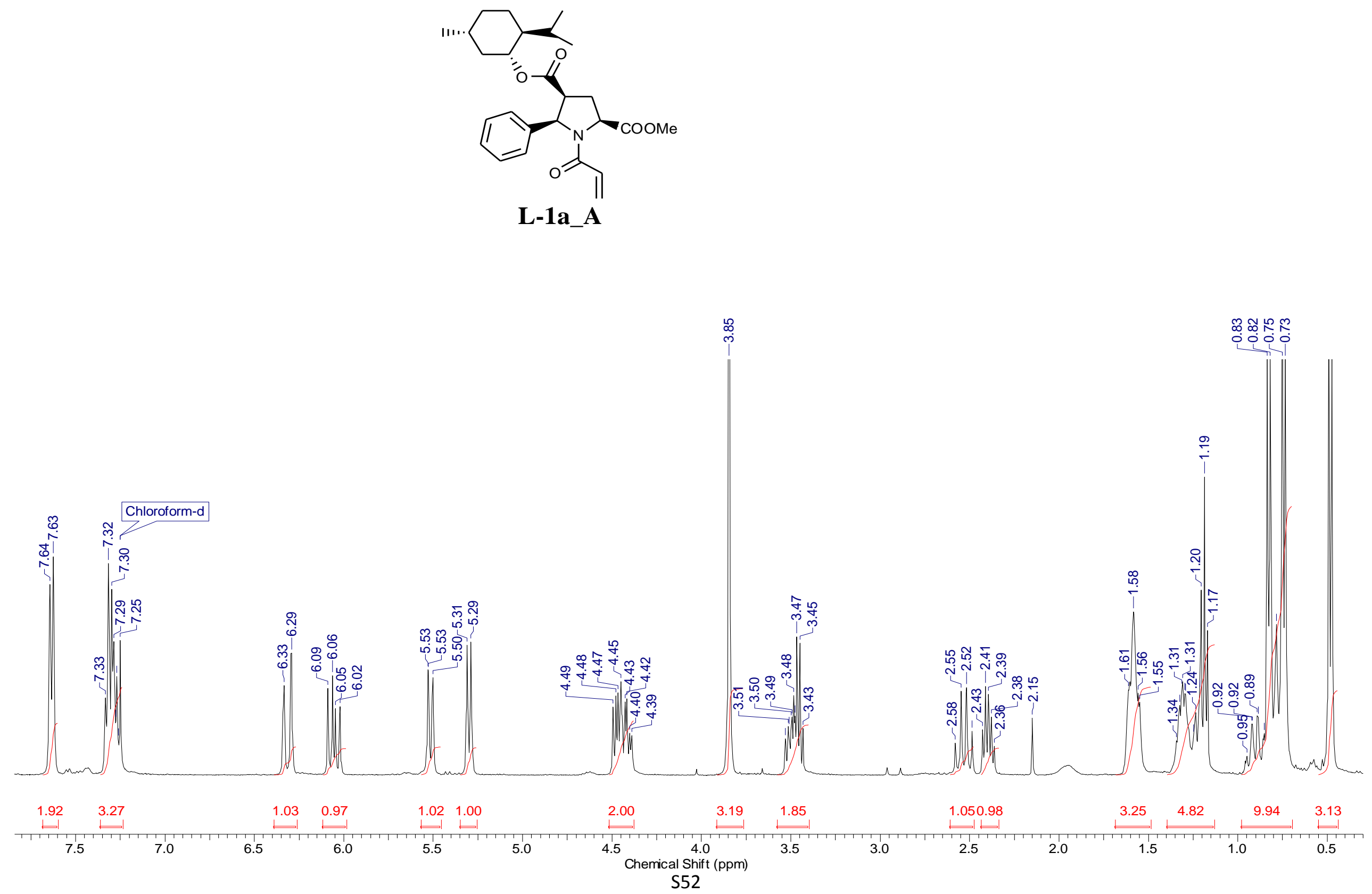


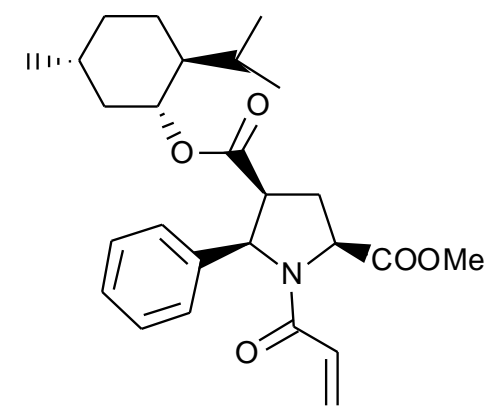

DMSO-d6

L-1a_A

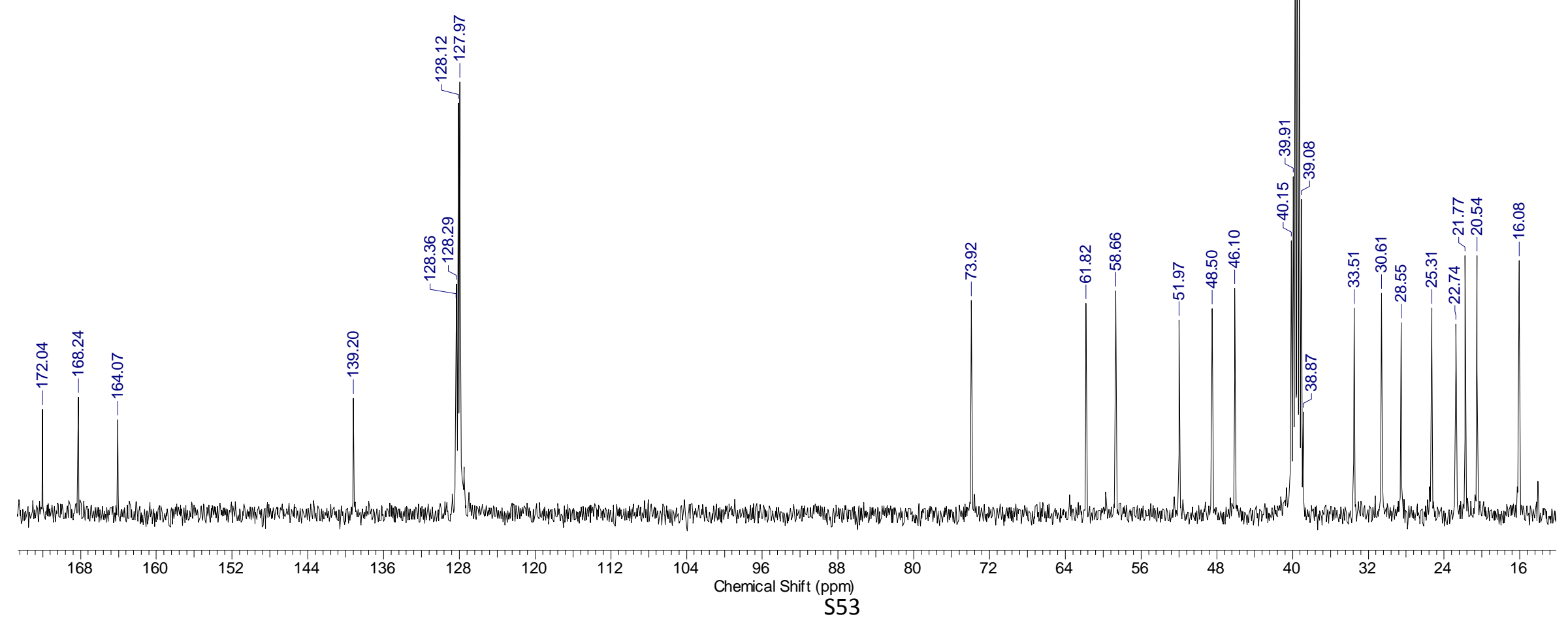



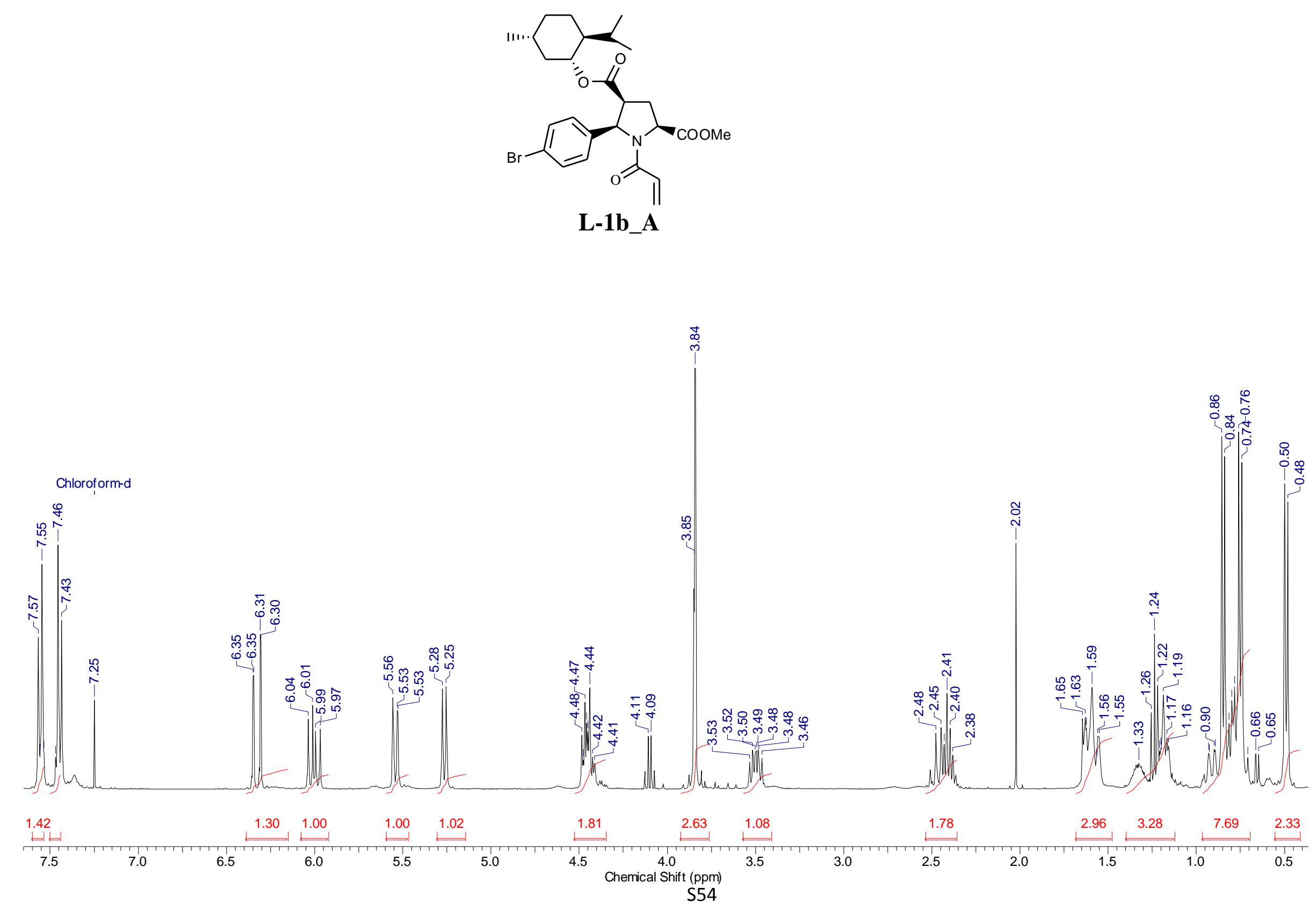
<smiles>CC(=O)[C@@H]1C[C@H](C(=O)O[C@H]2C[C@H](C)CC[C@H]2C(C)C)[C@H](c2ccc(Br)cc2)N1C(=O)C=C[13CH3]</smiles>

L-1b_A

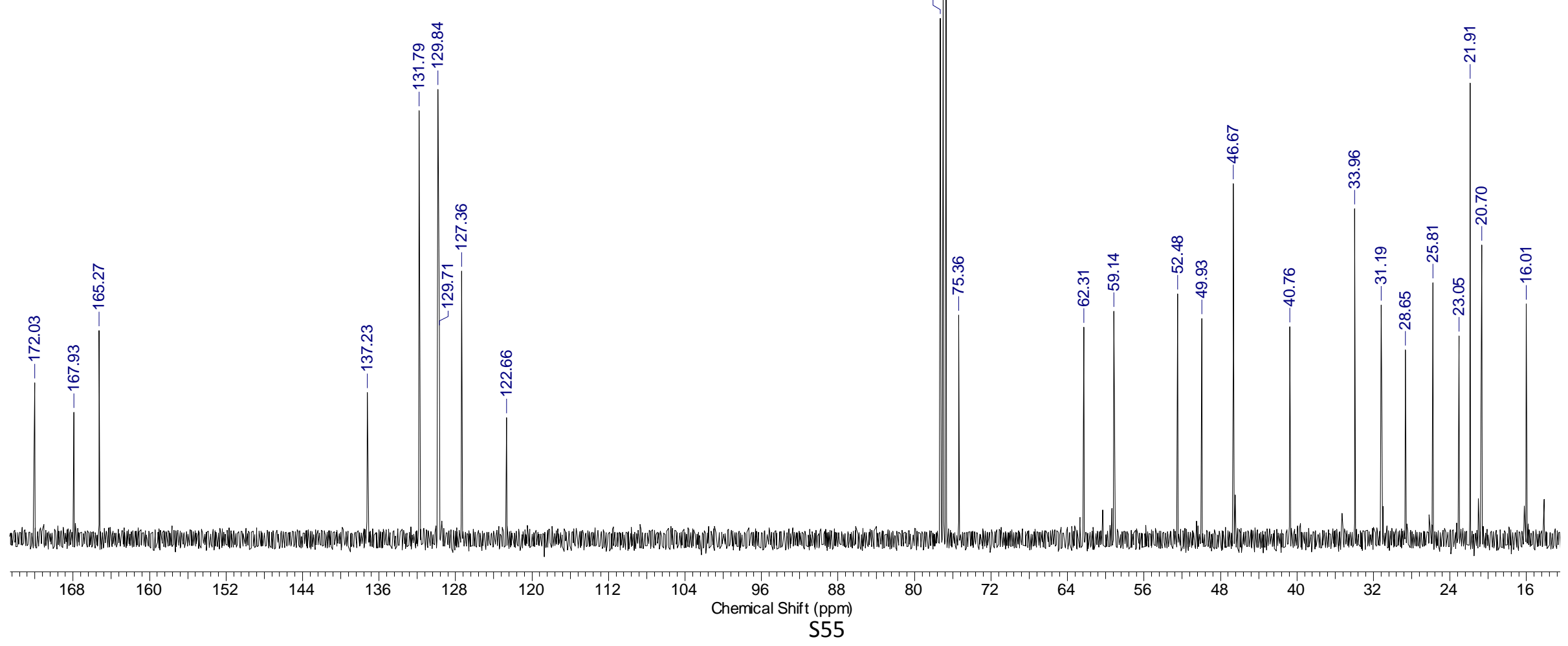



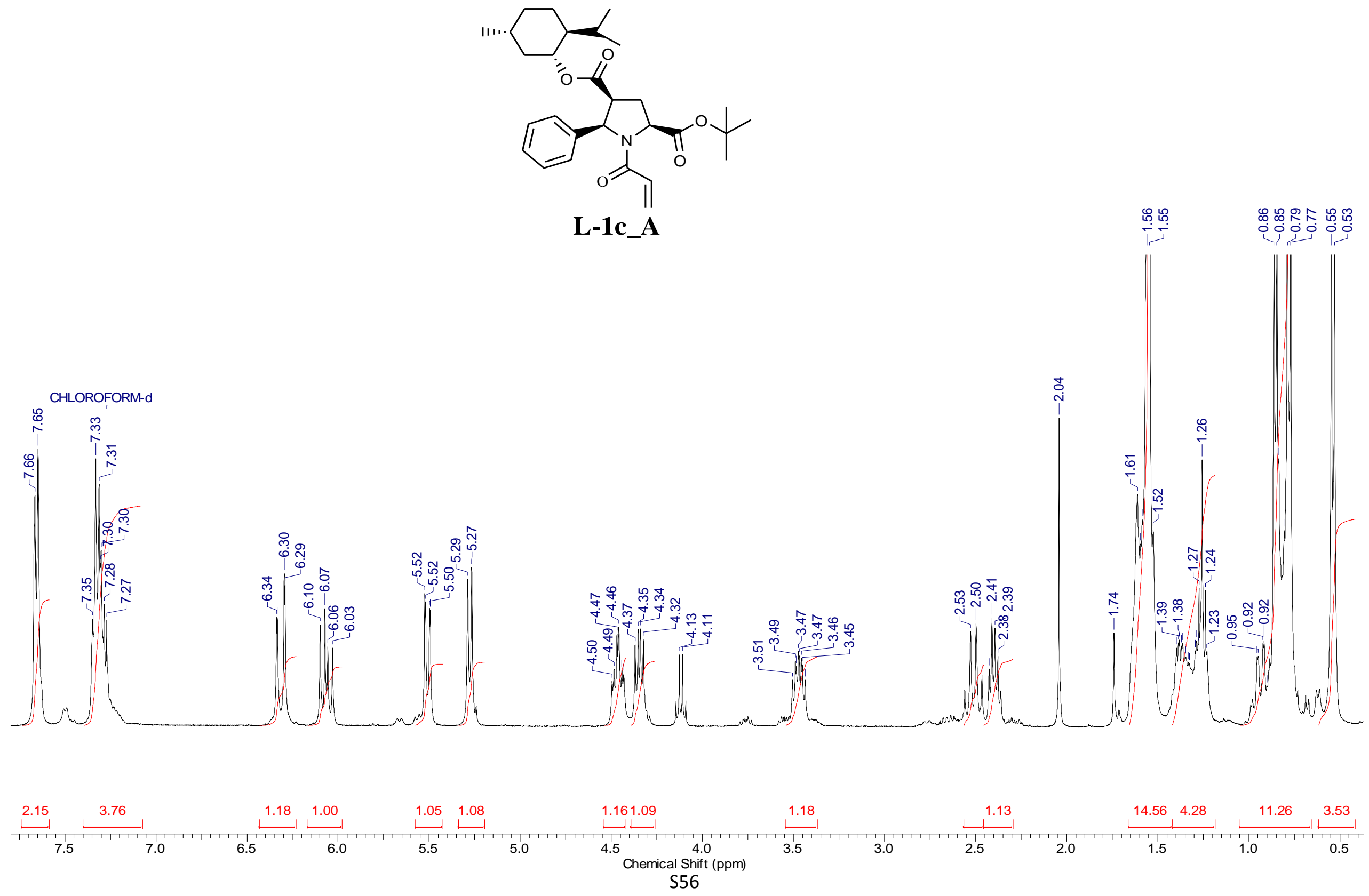

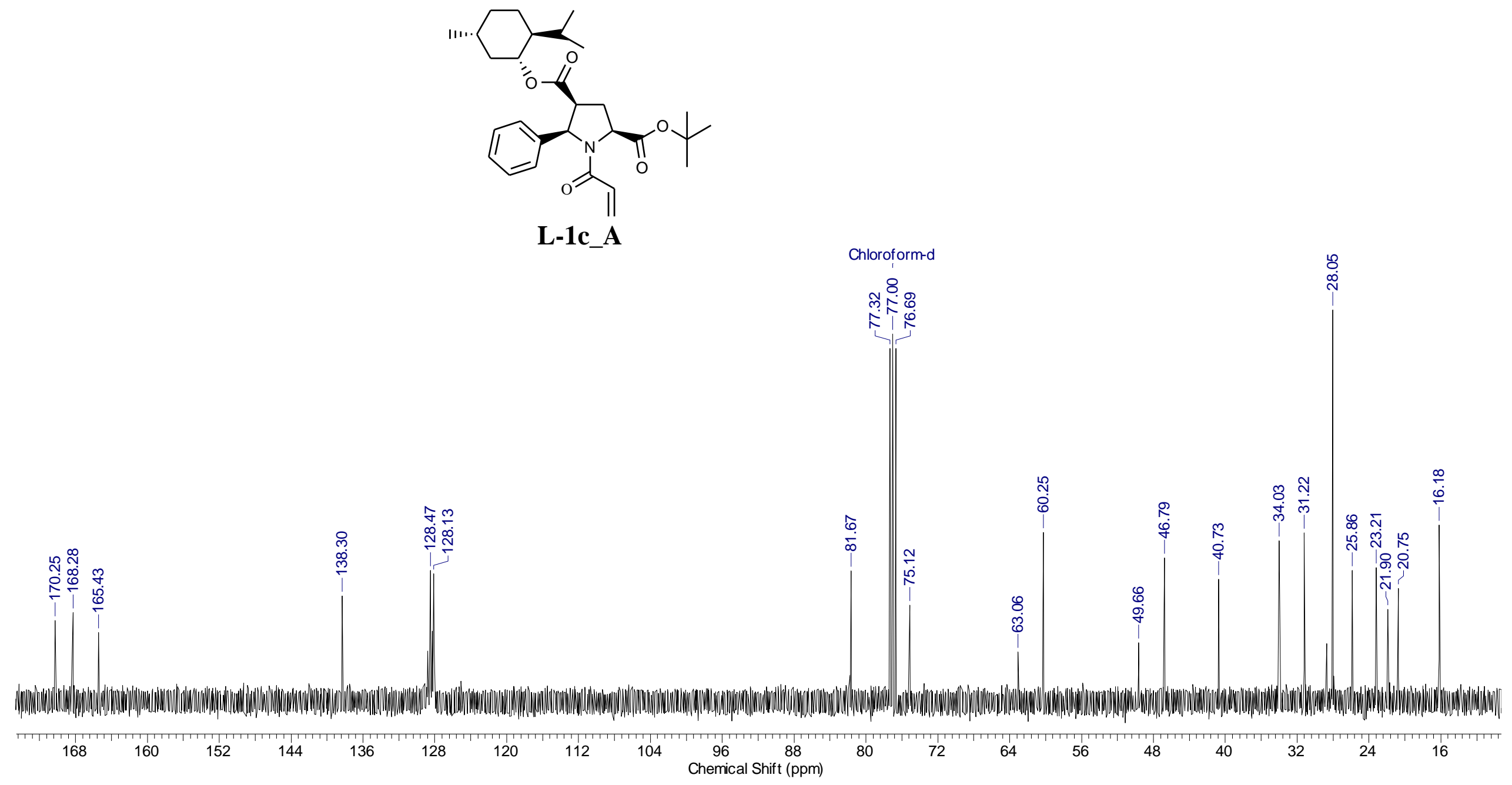


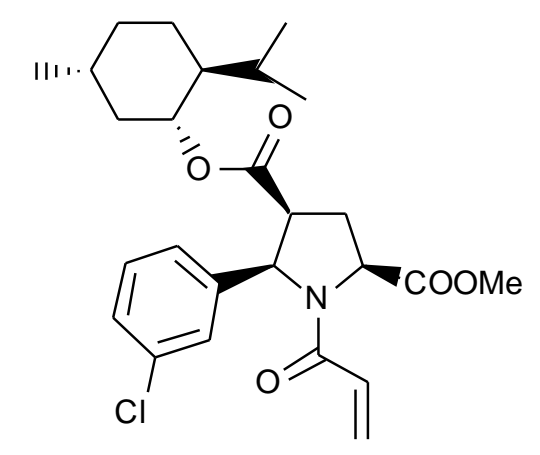

L-1d A
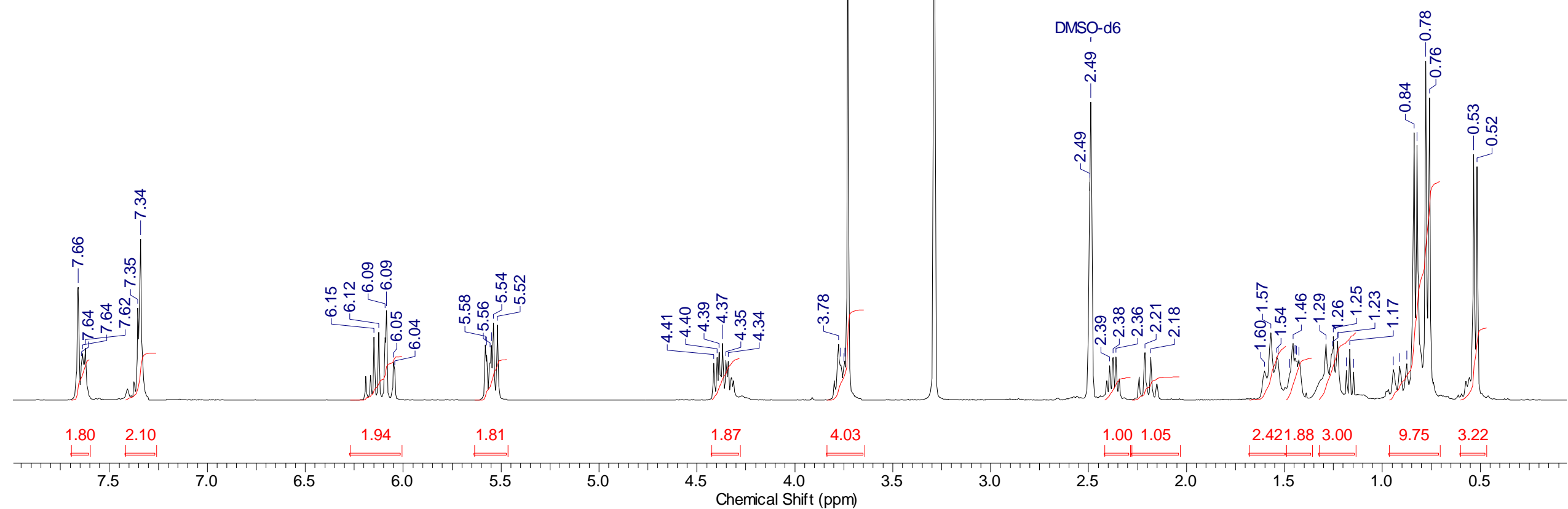

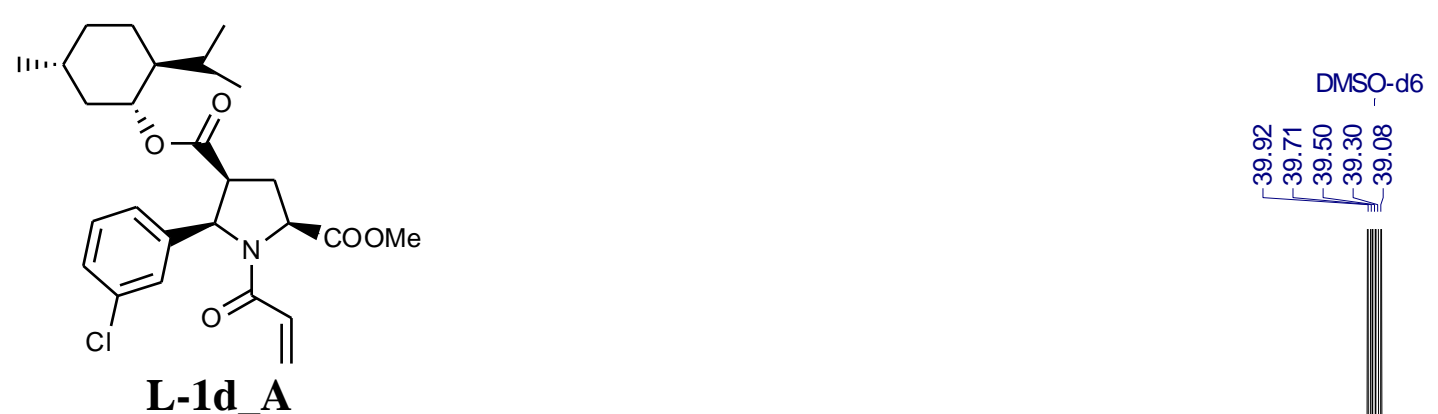

L-1d_A

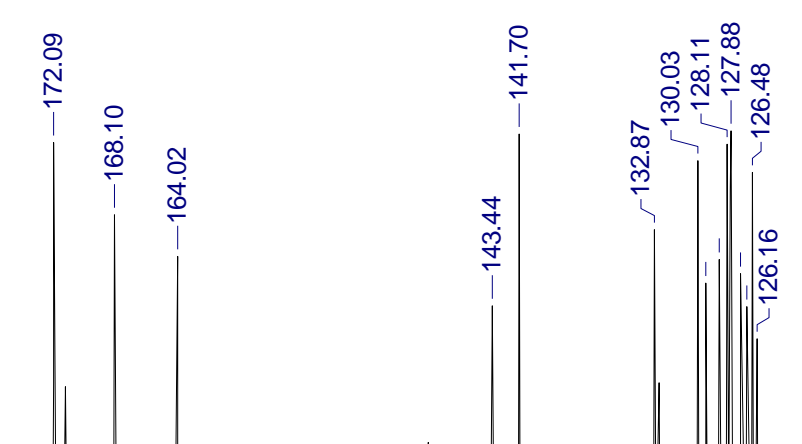

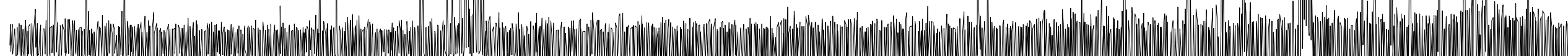

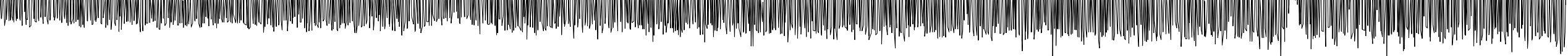

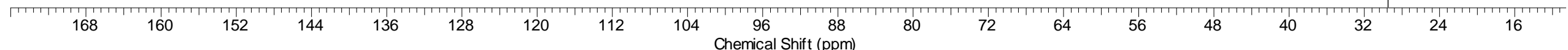



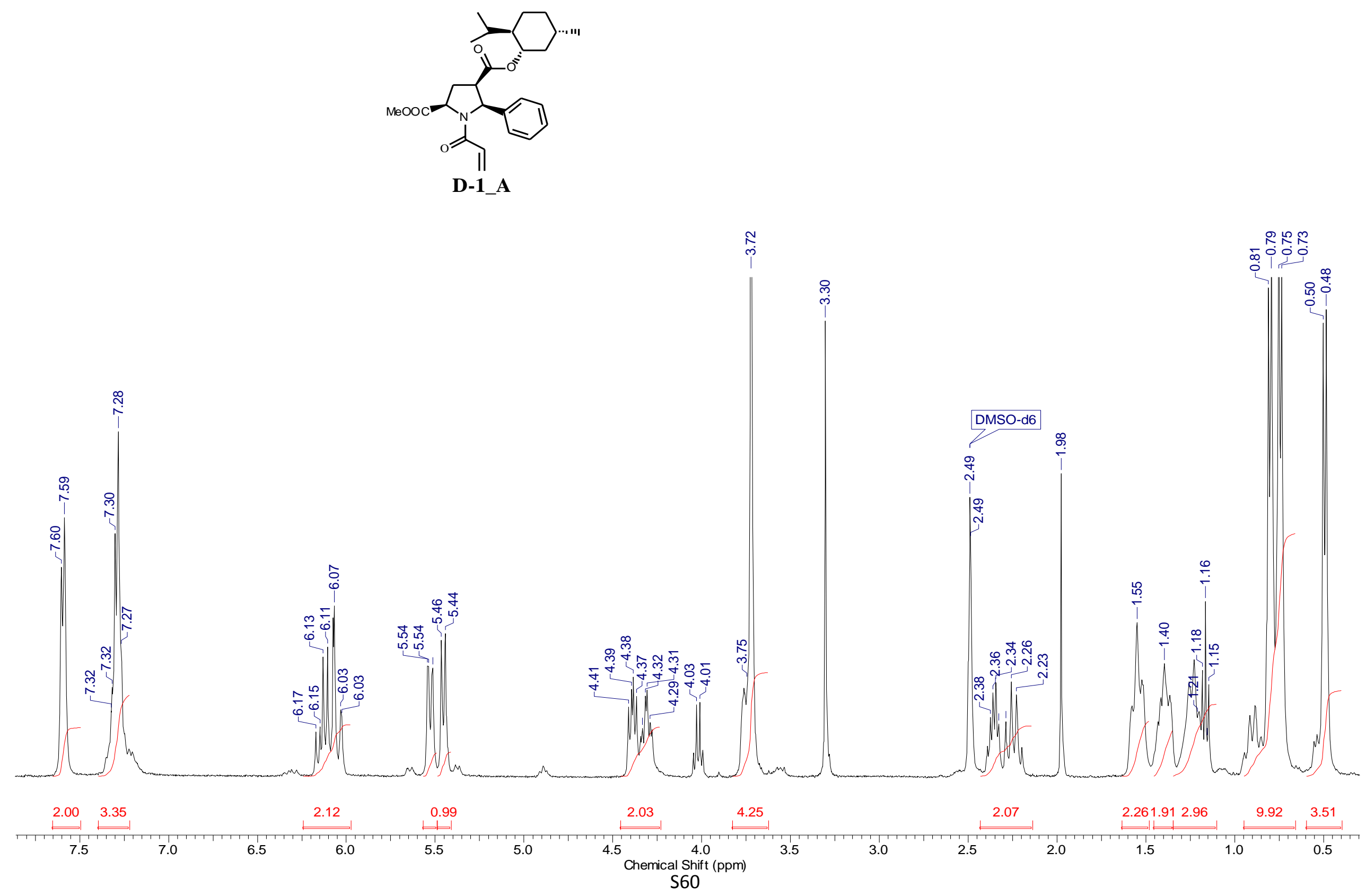

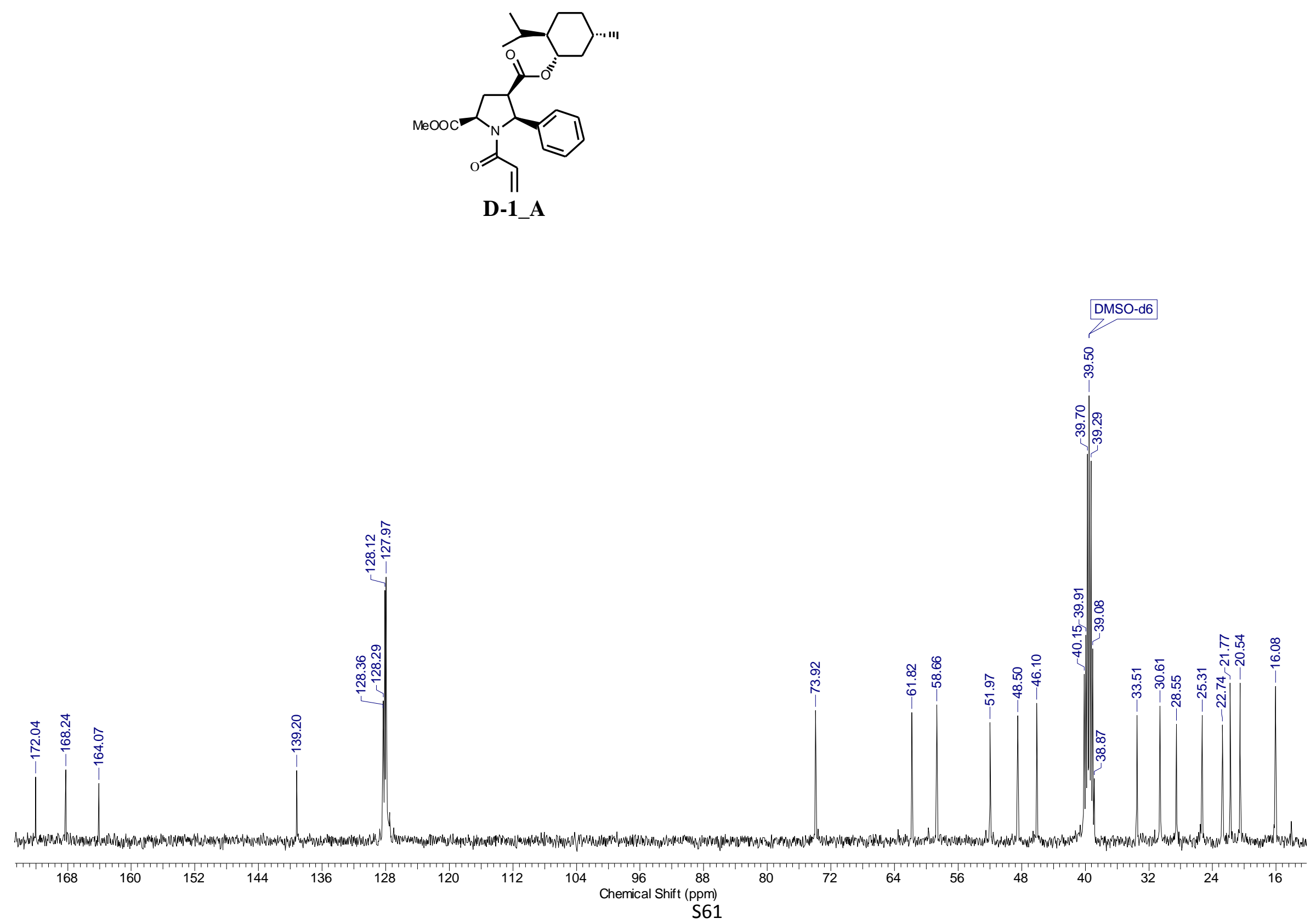

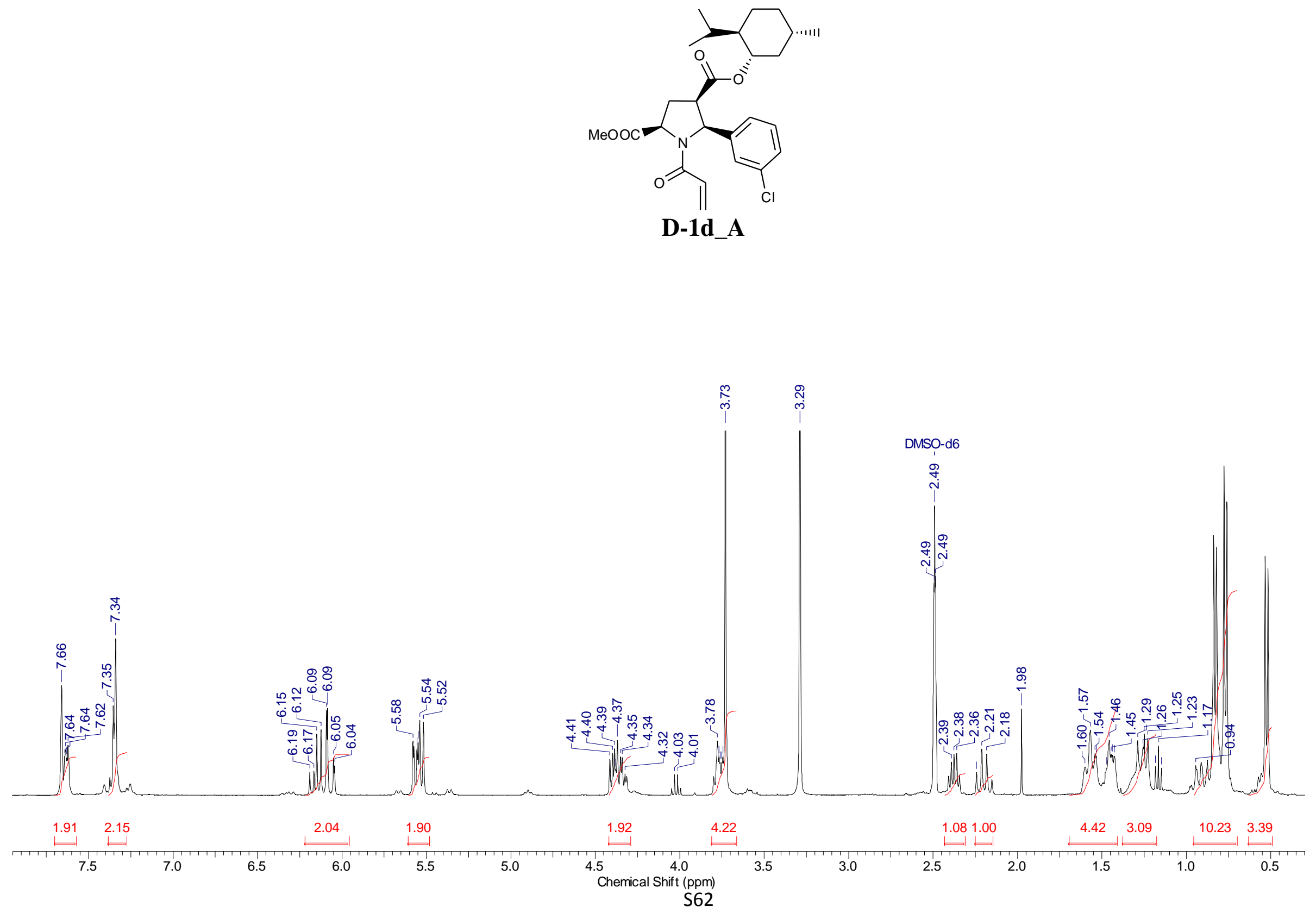

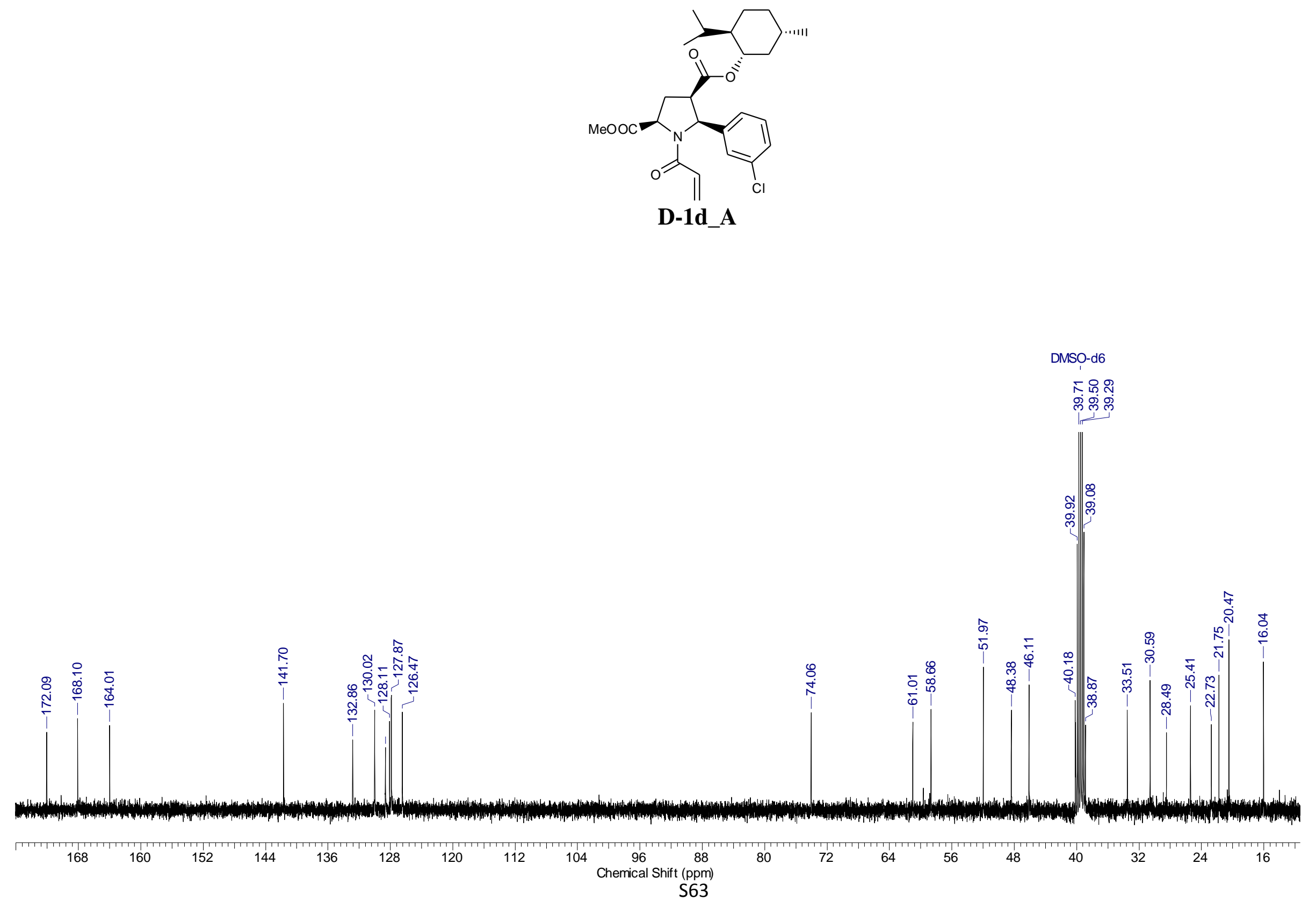

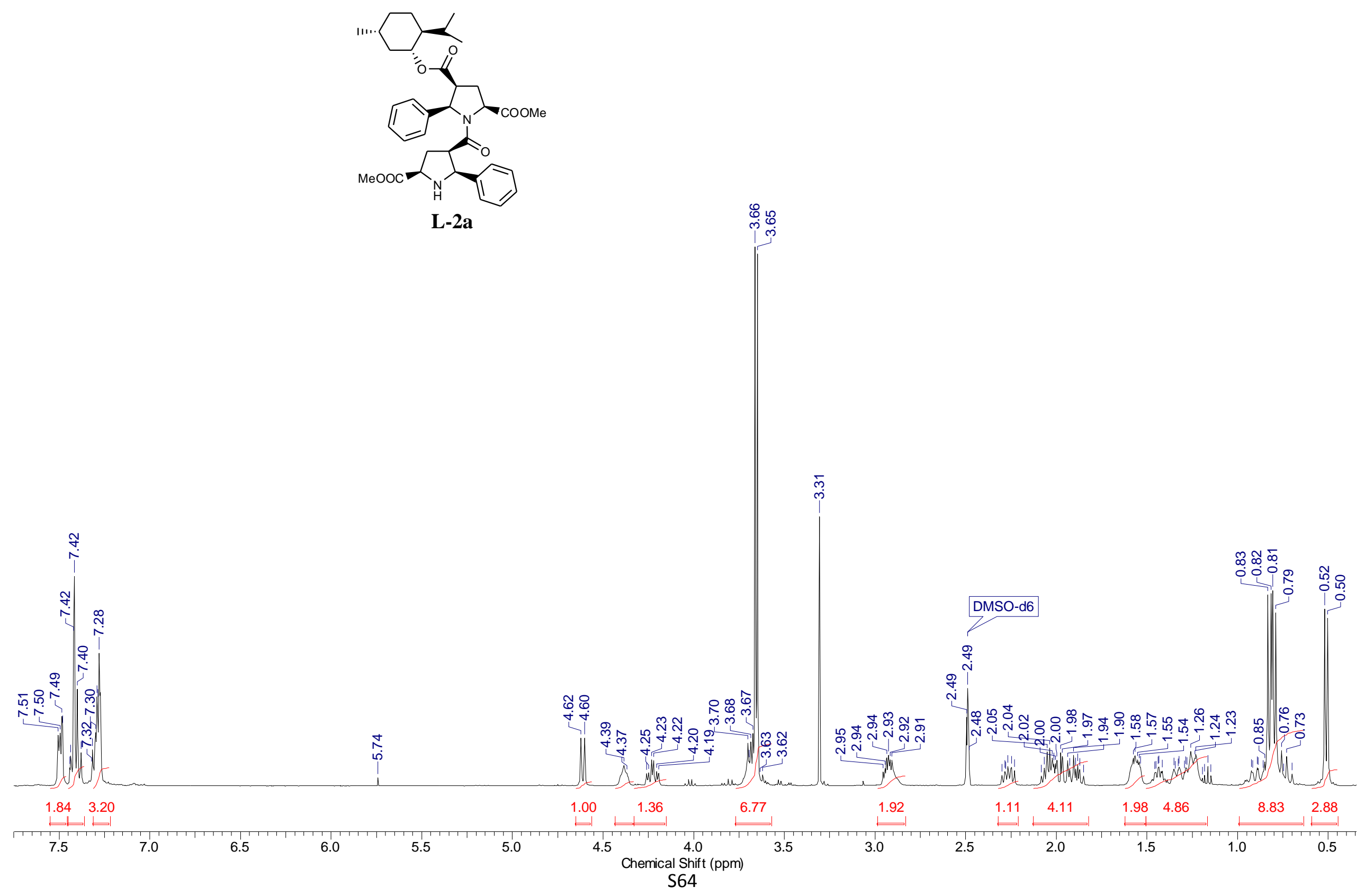


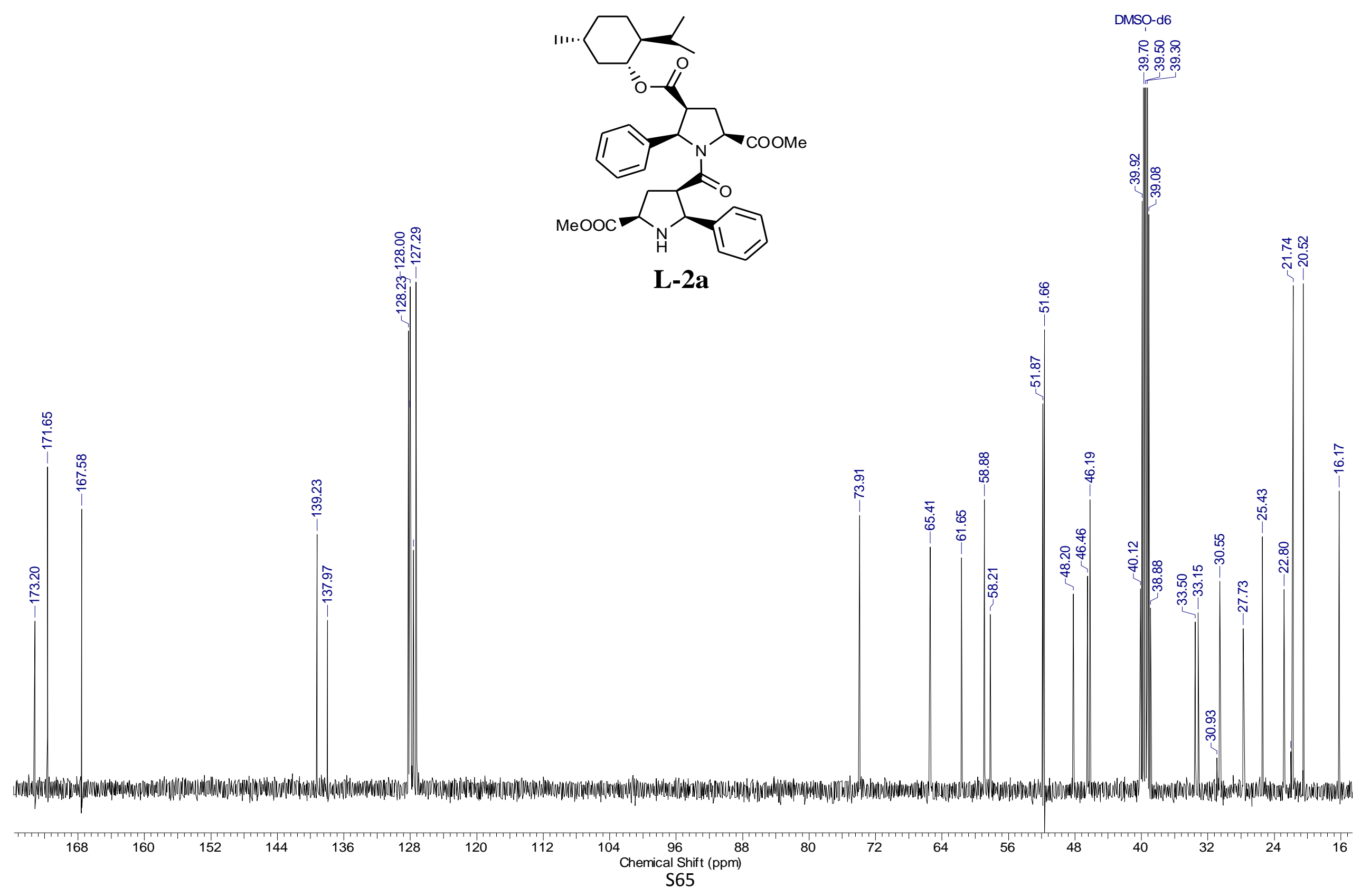



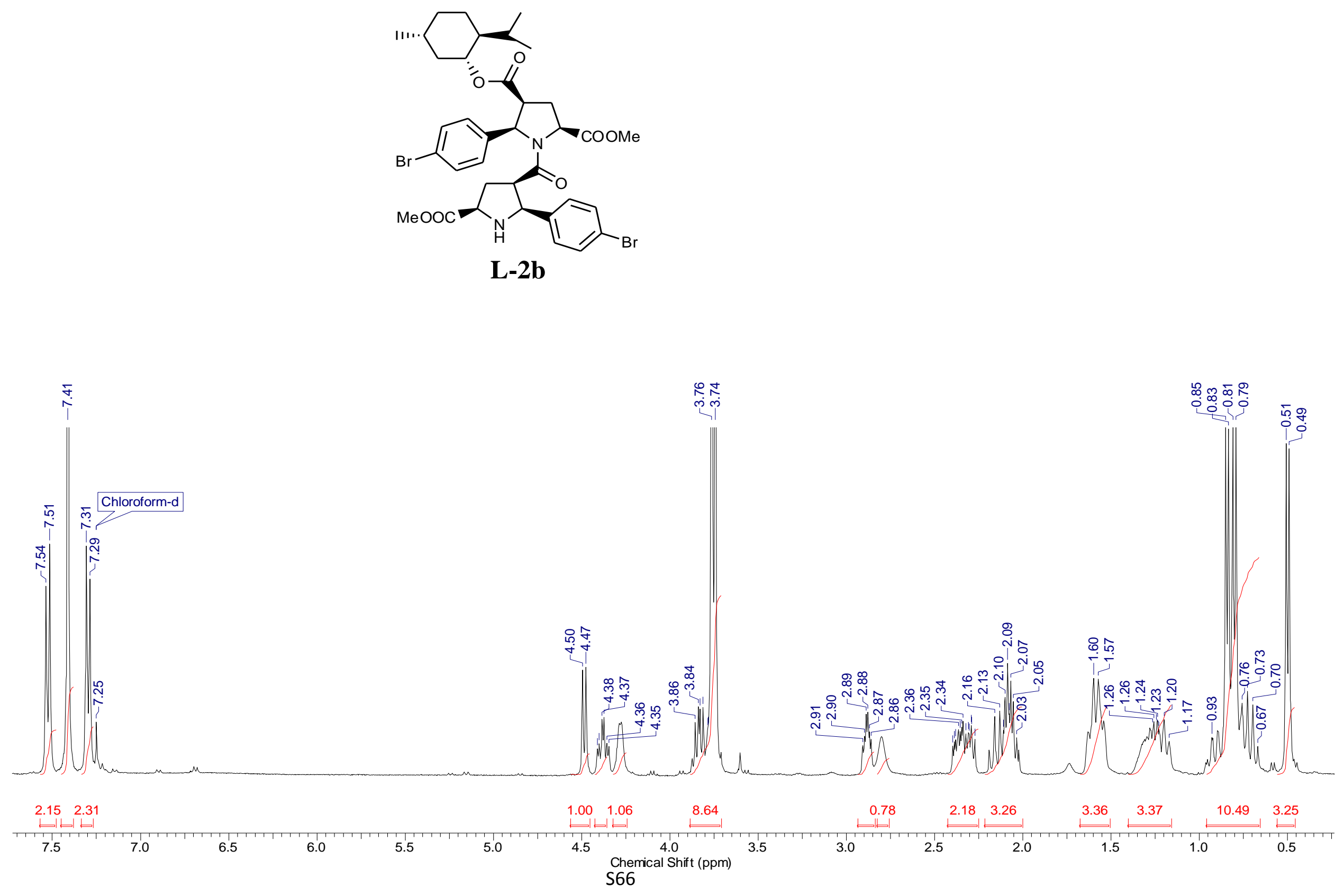


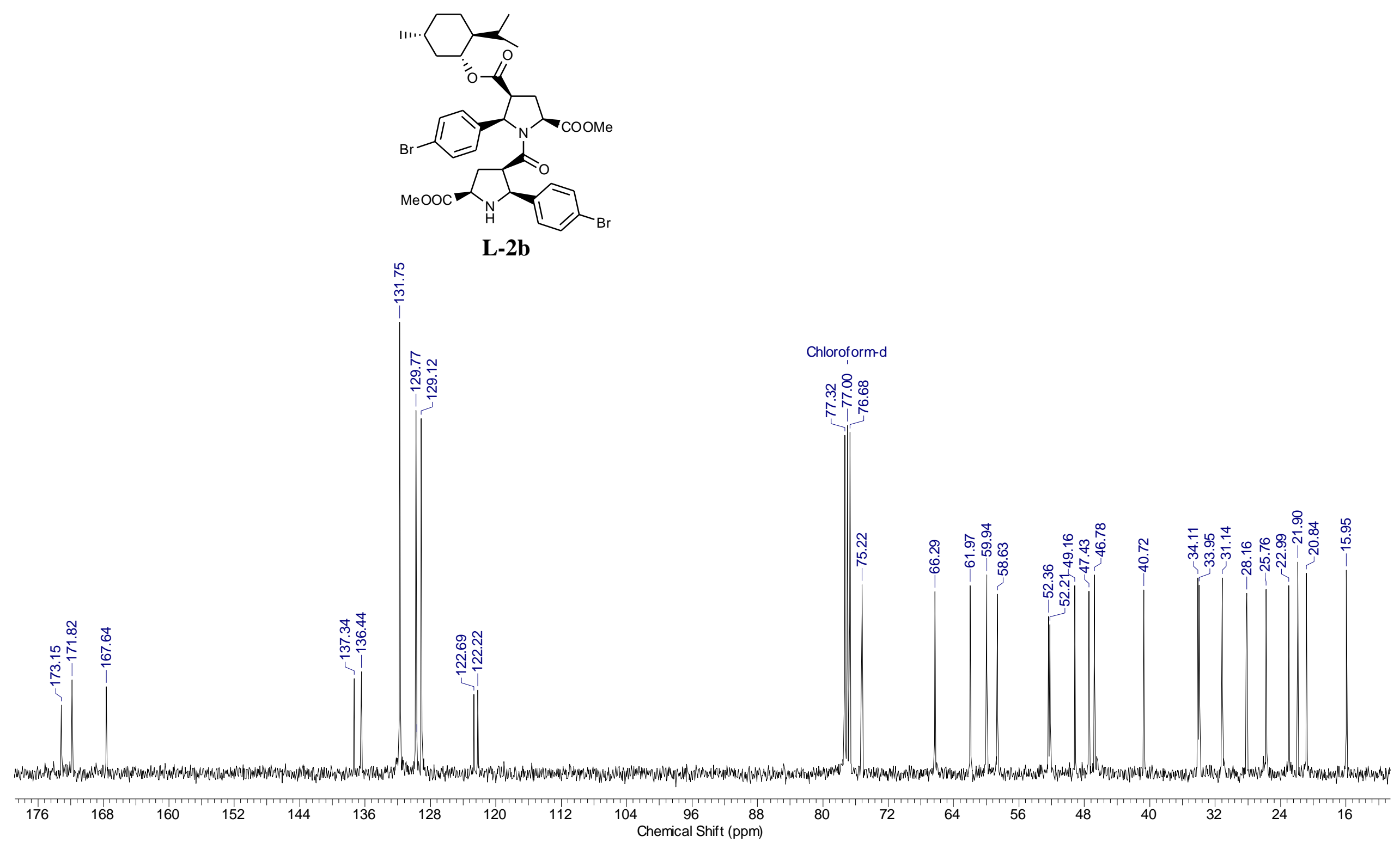




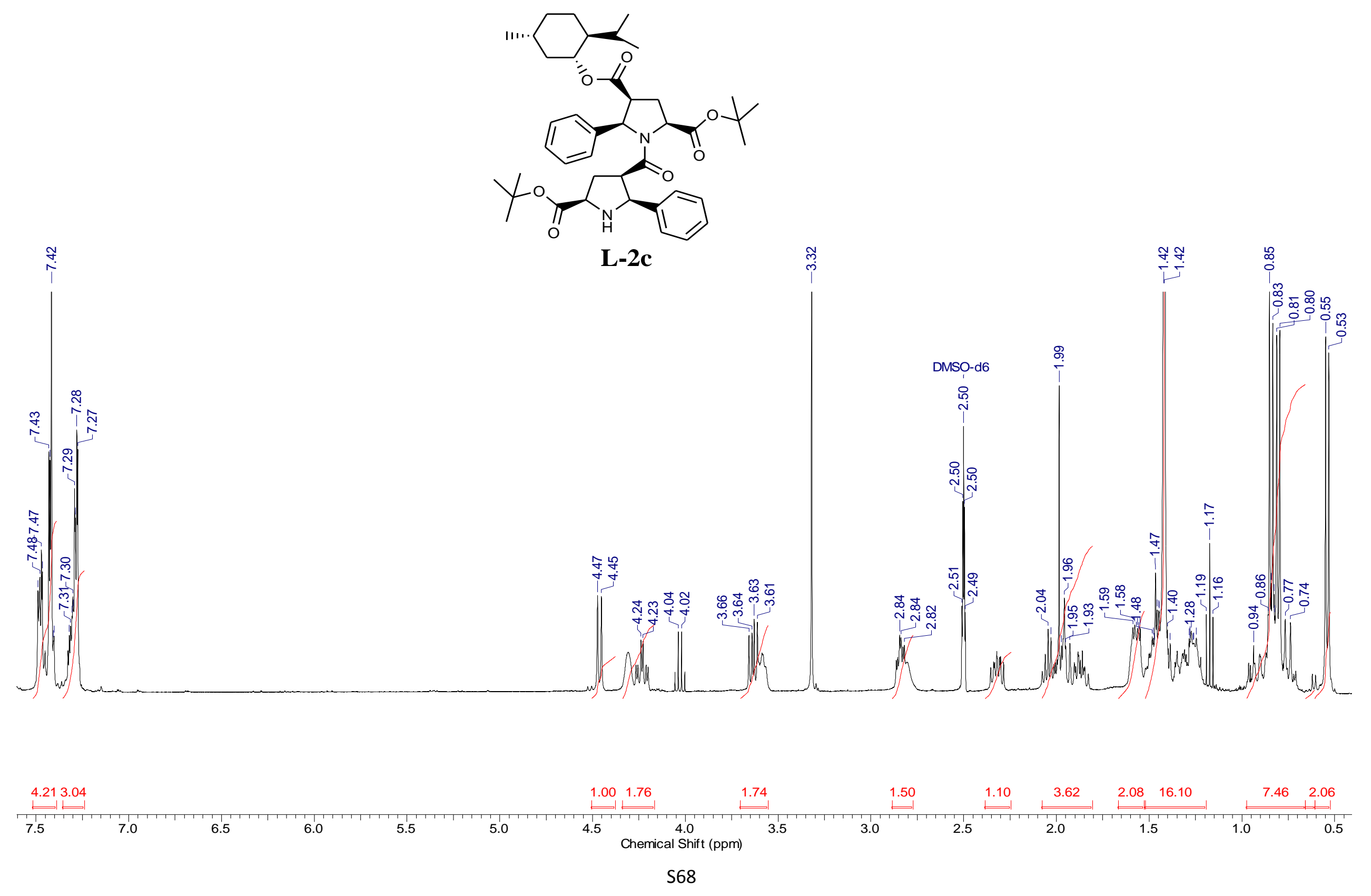




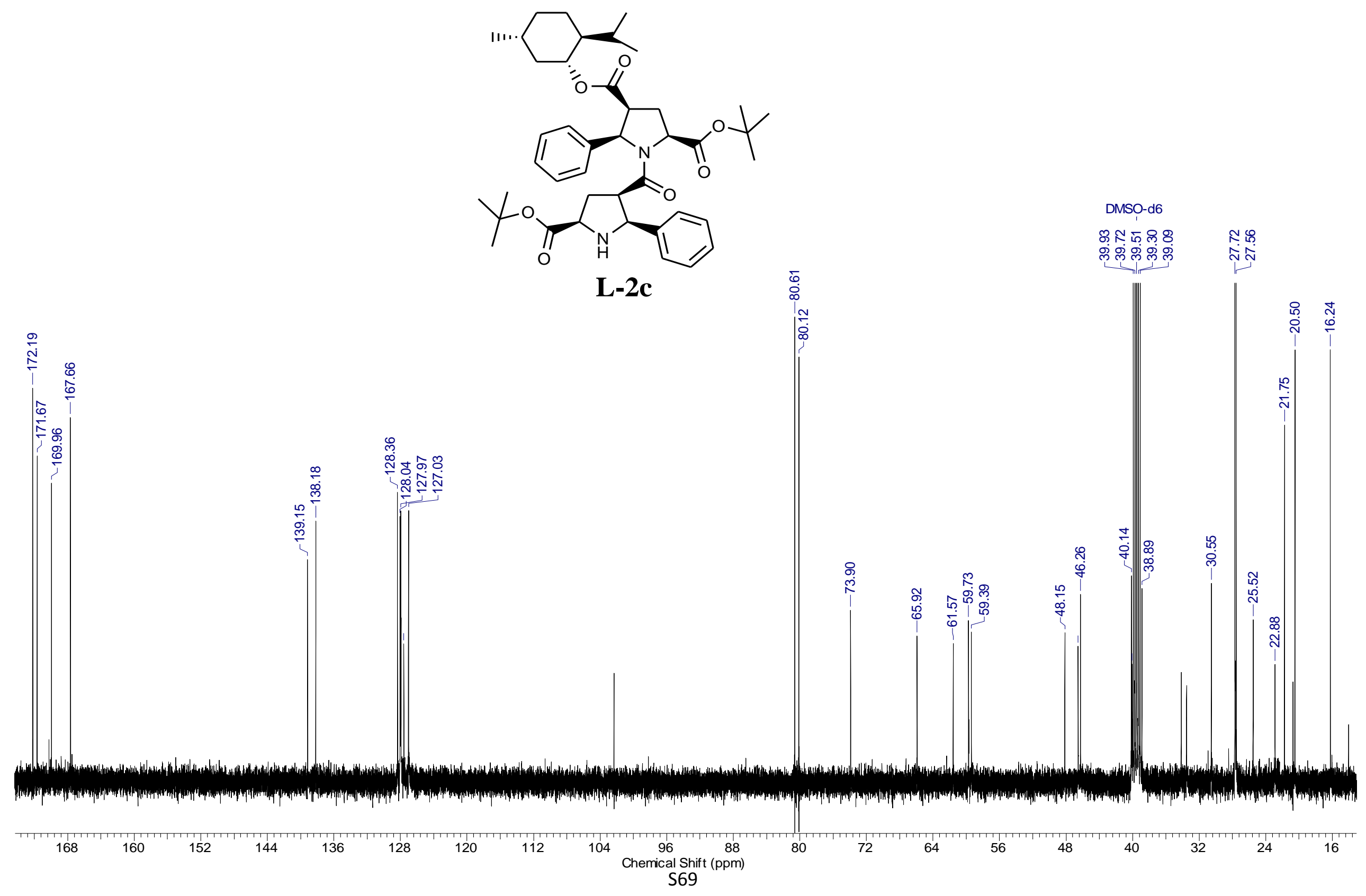



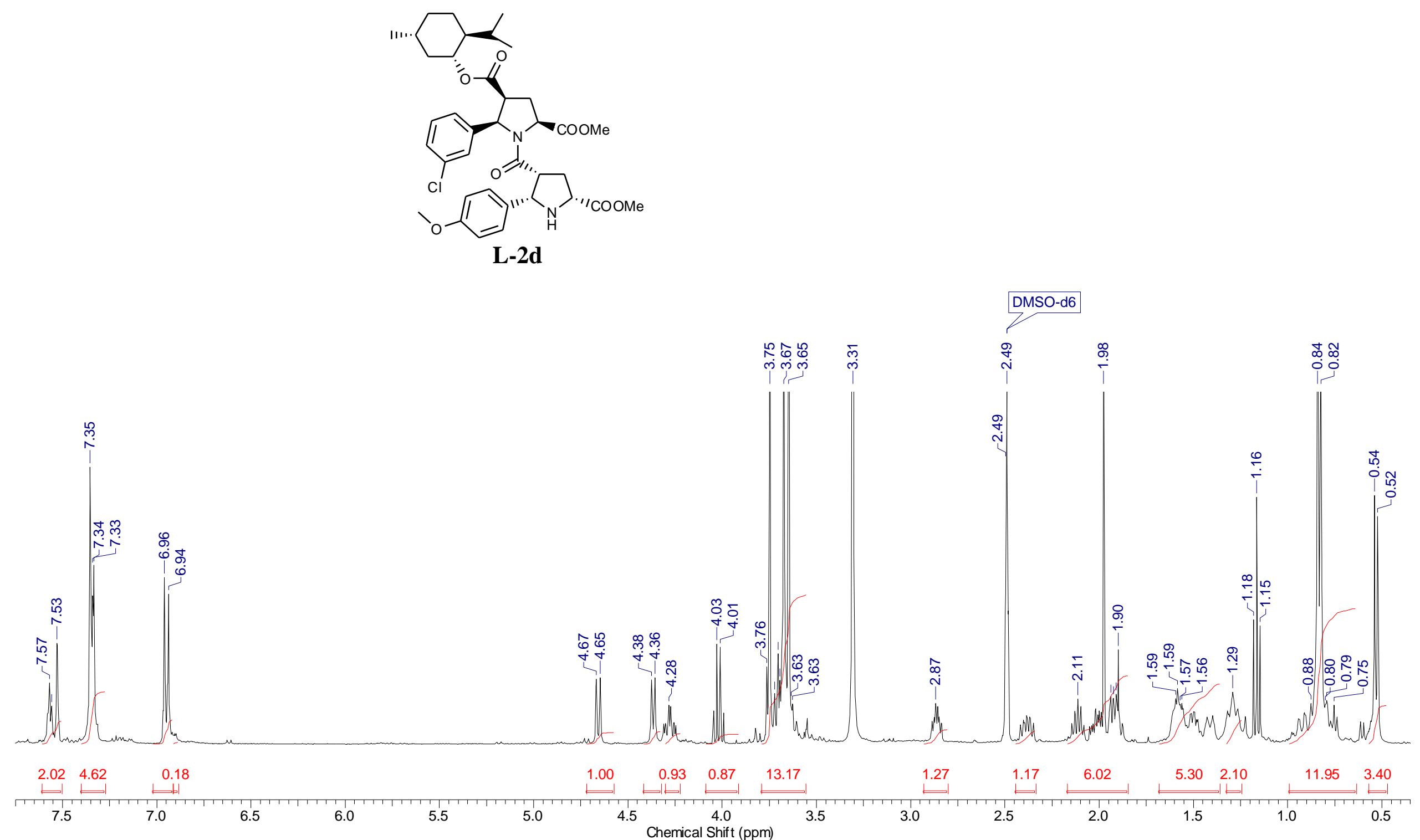


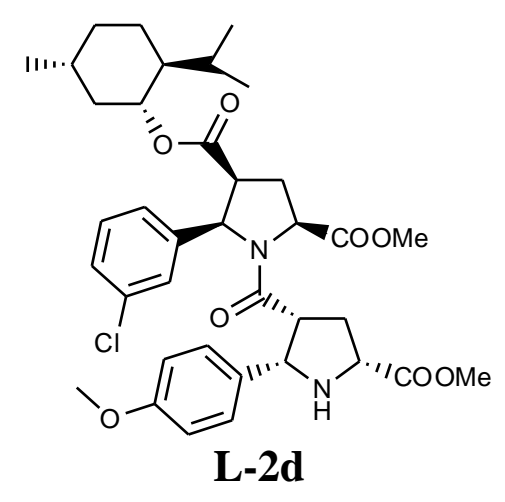

DMSO-d6

주요

。.|

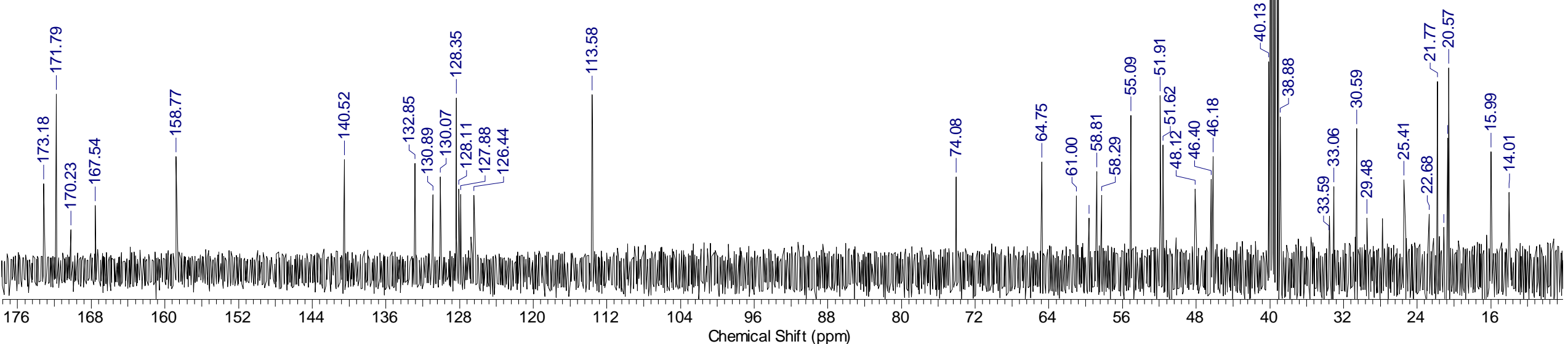



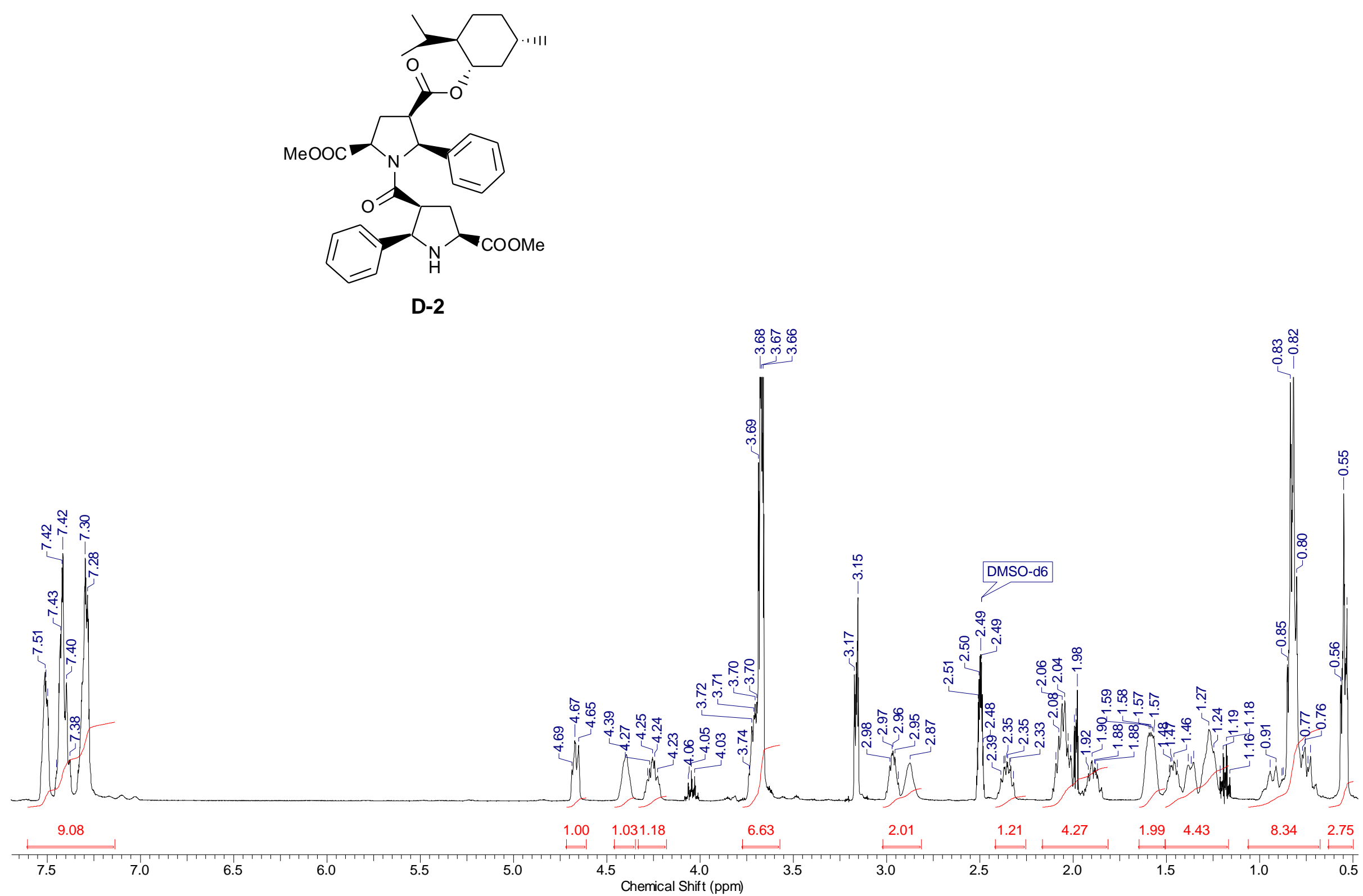


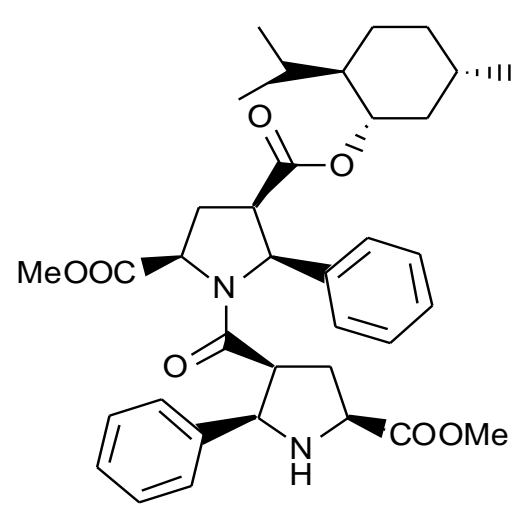

D-2

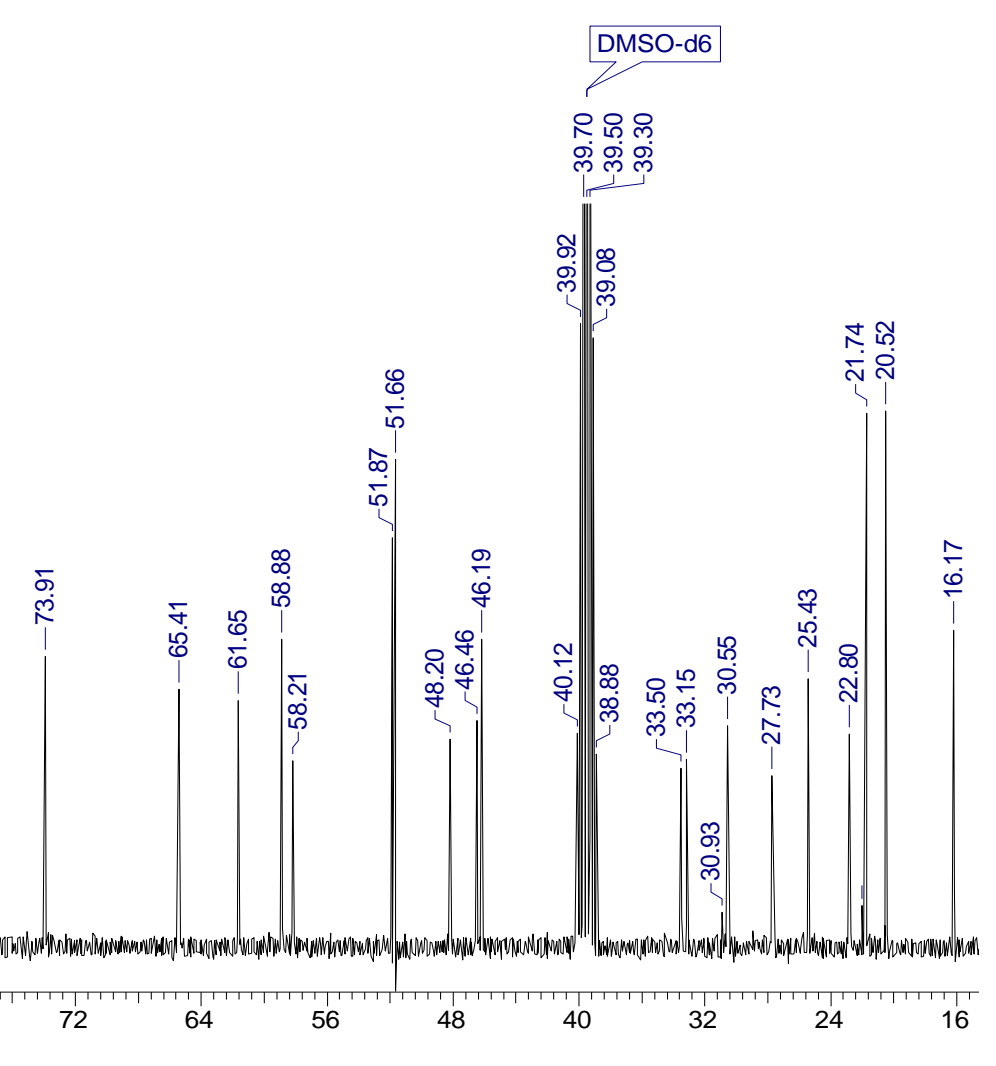



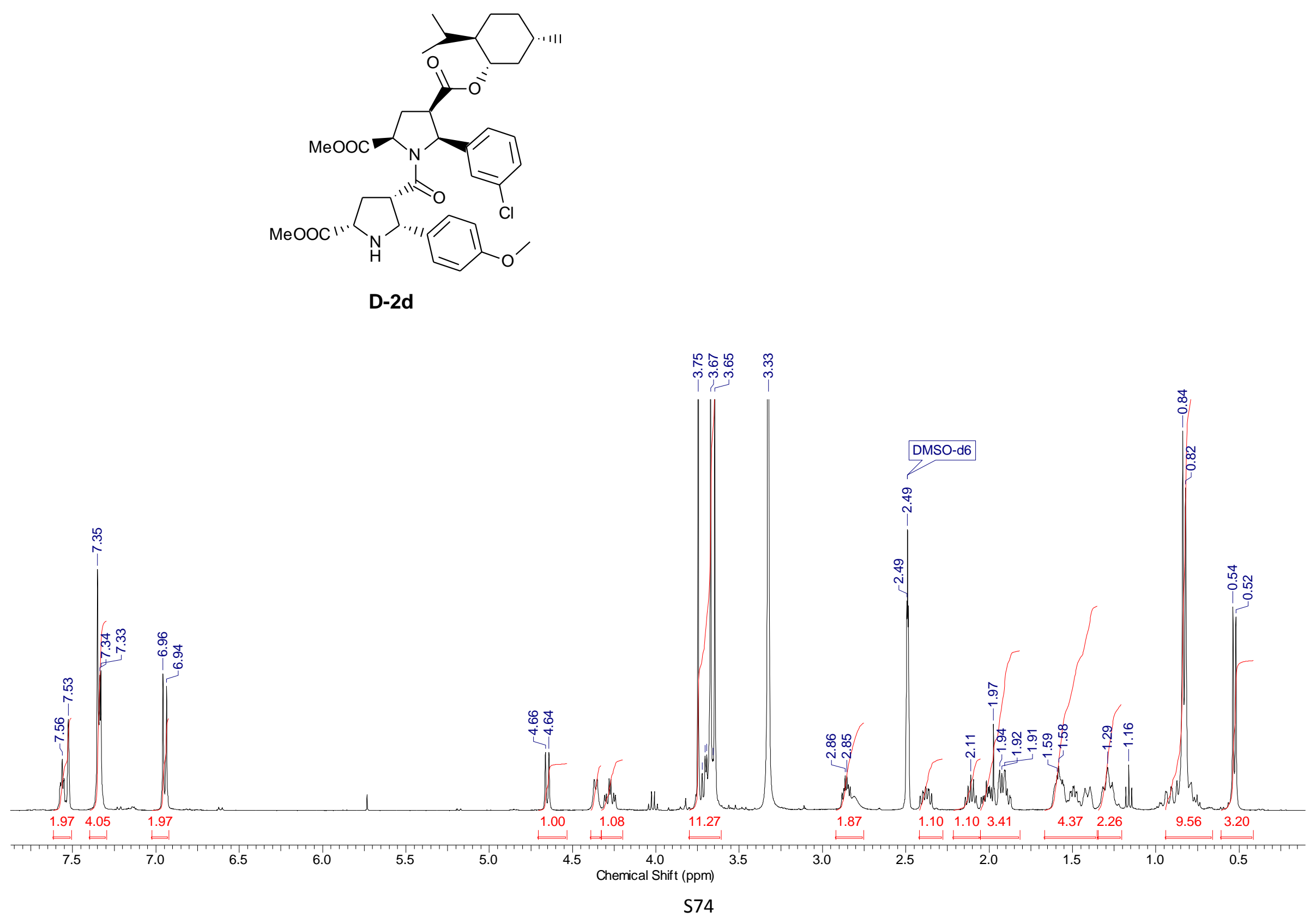


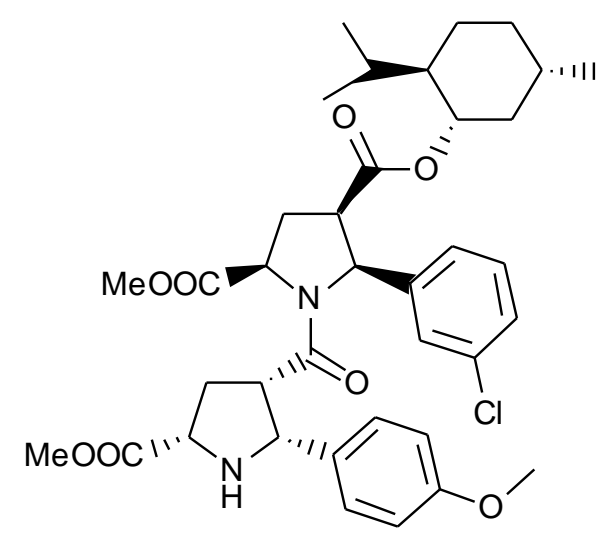

D-2d

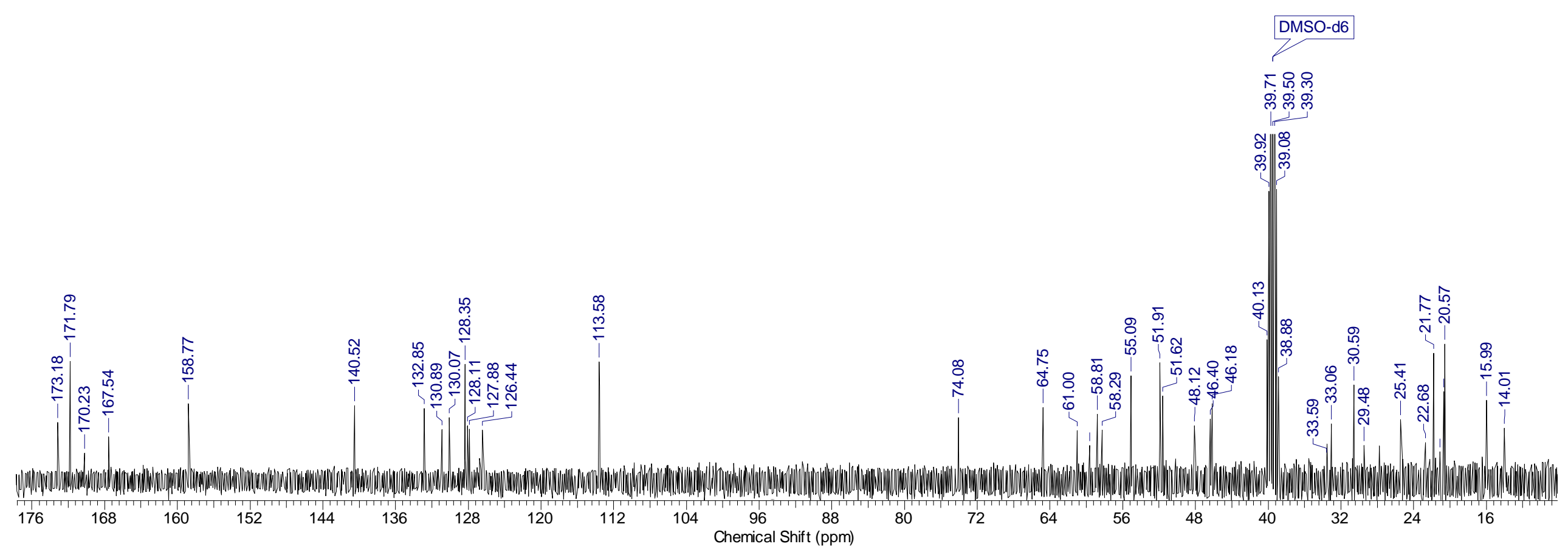



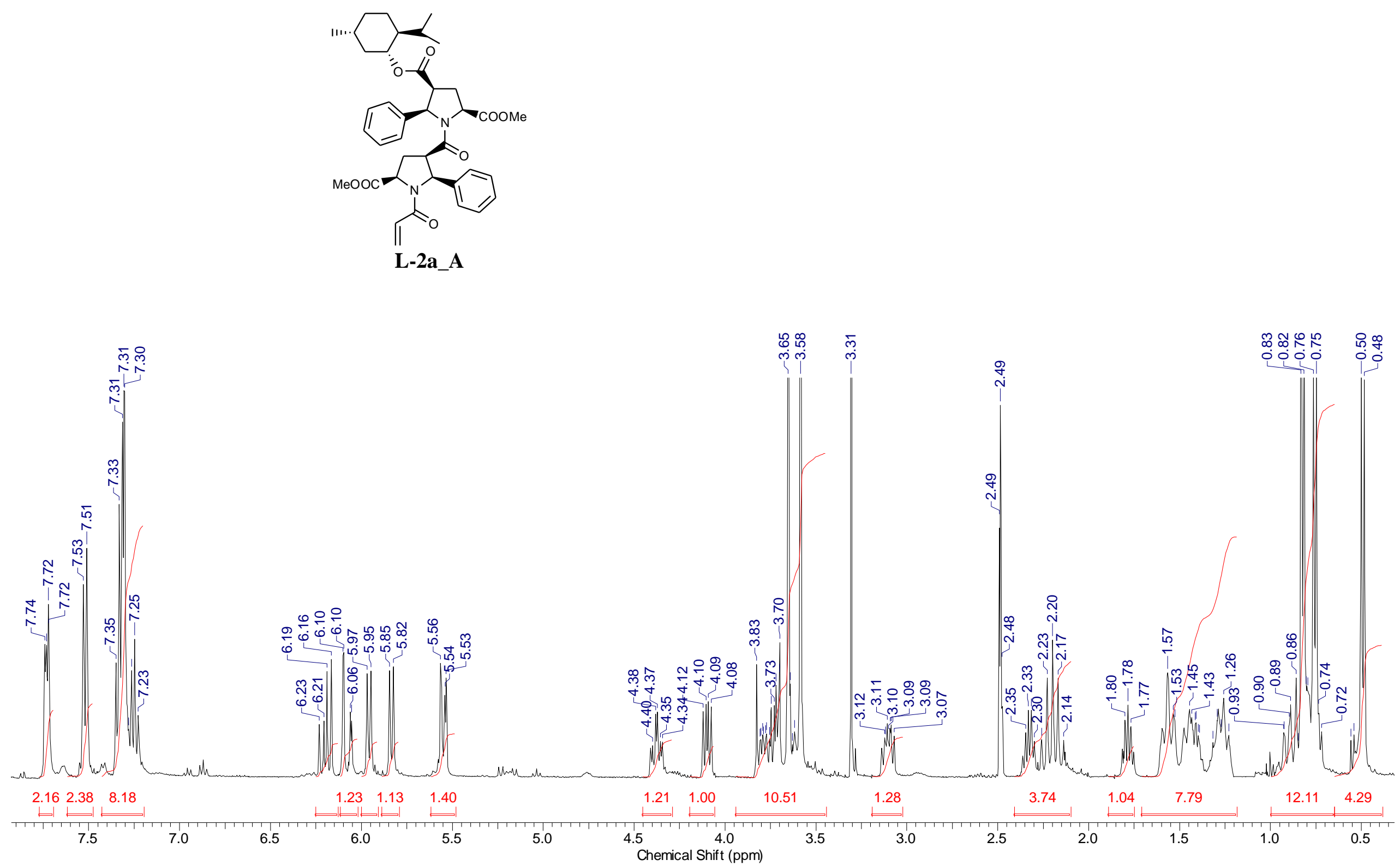

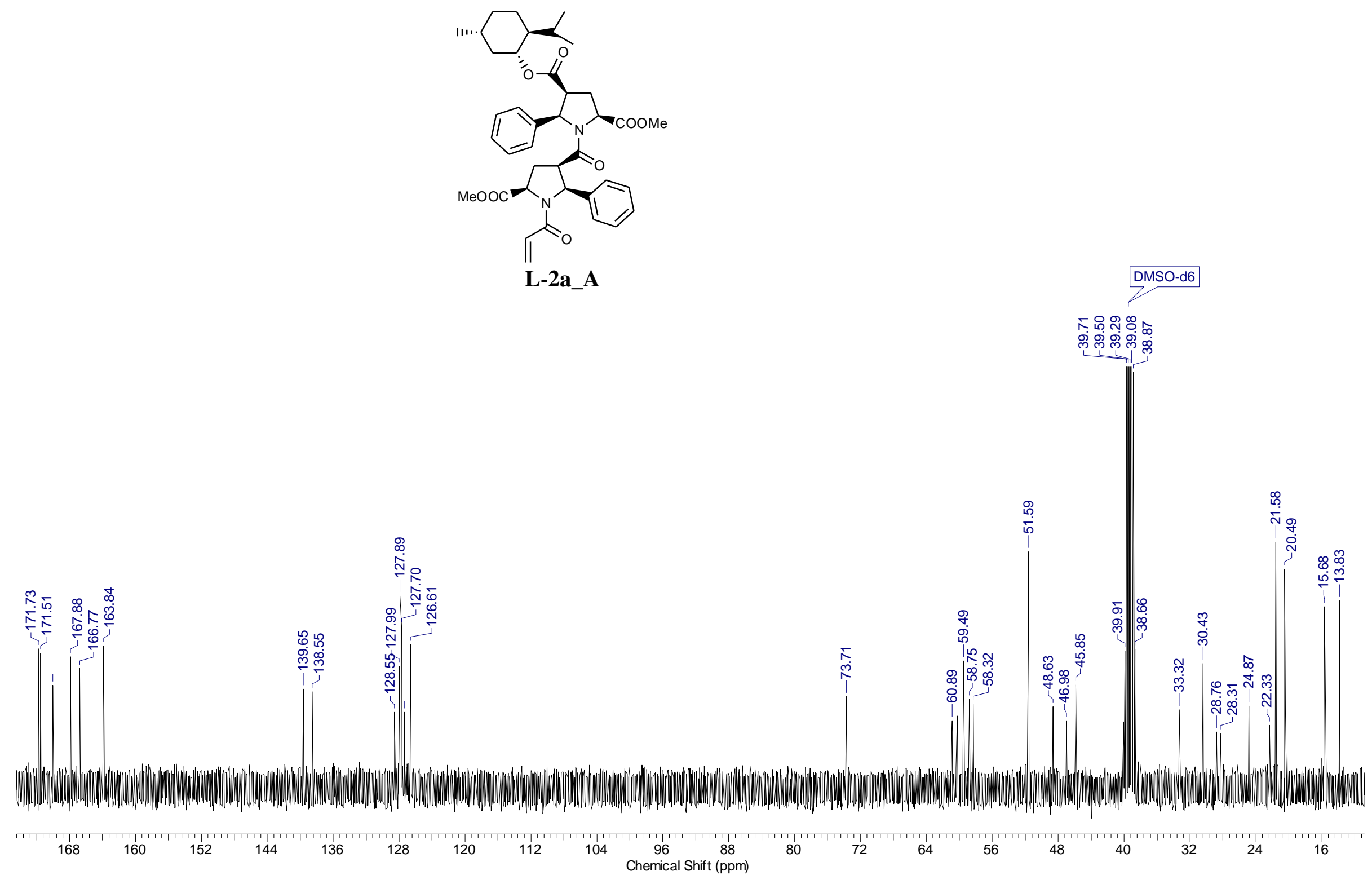

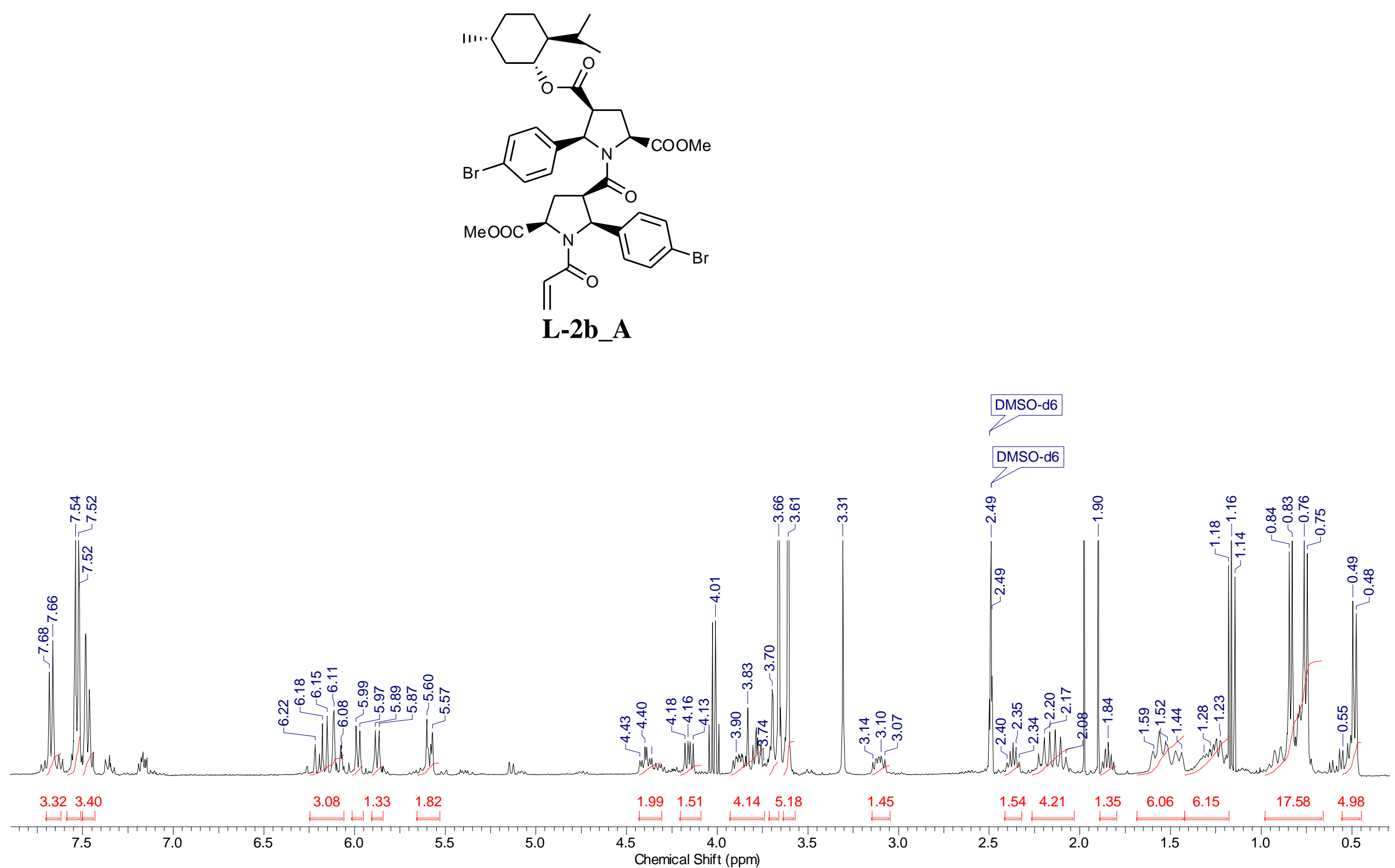


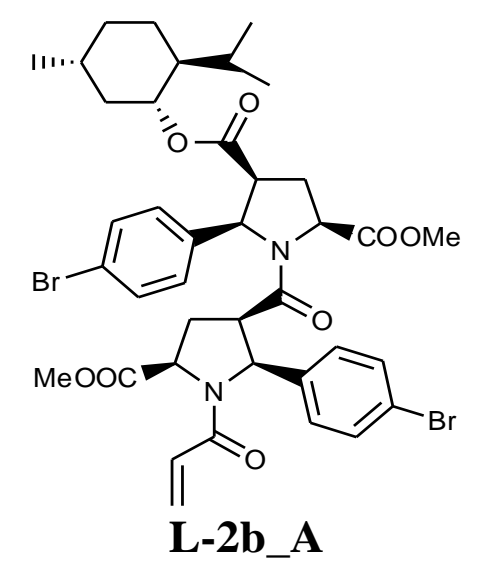

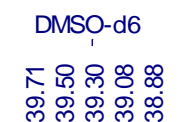
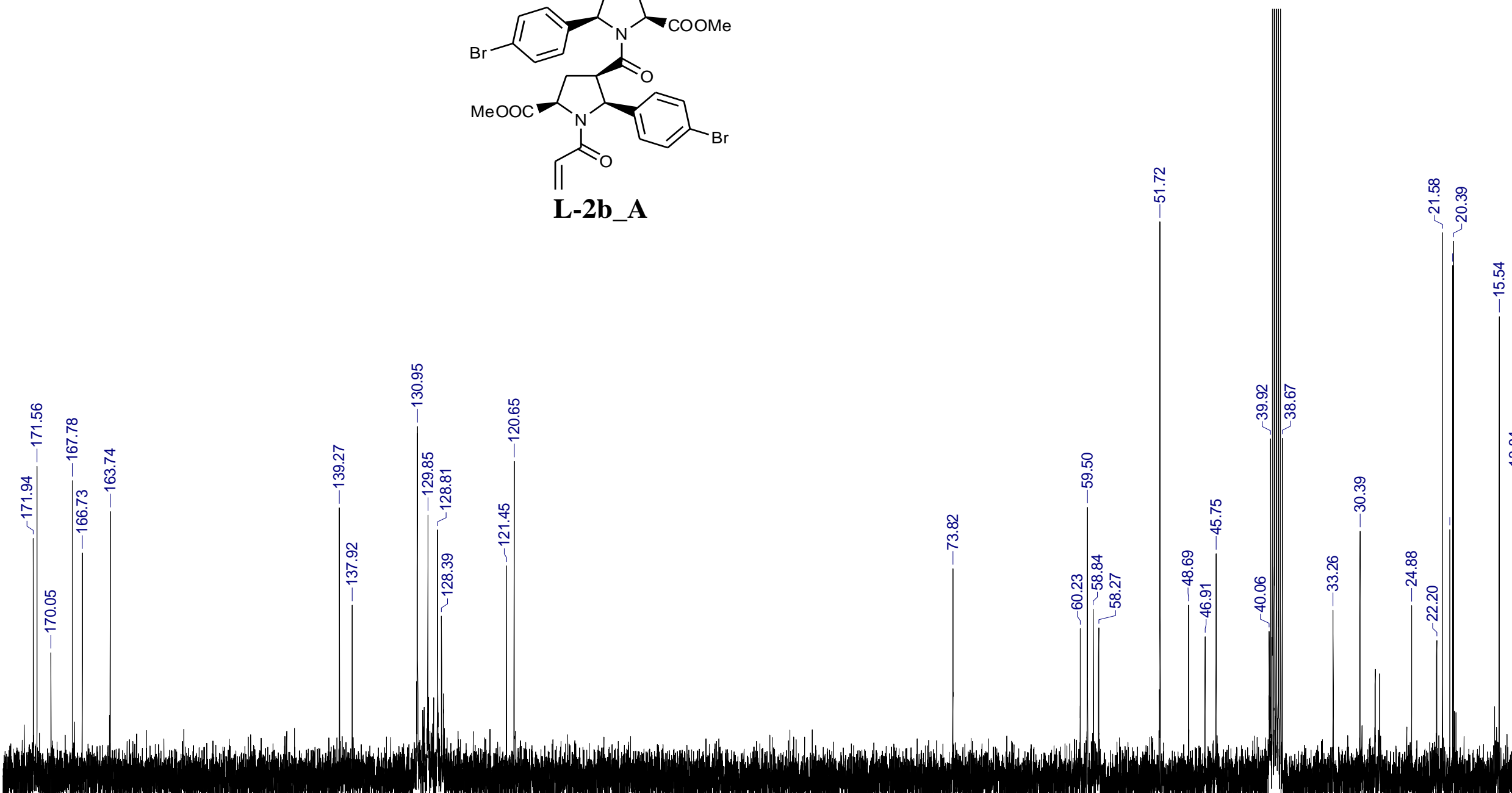

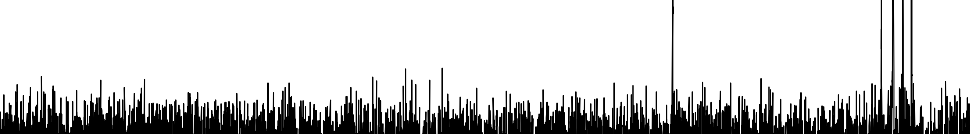
管 

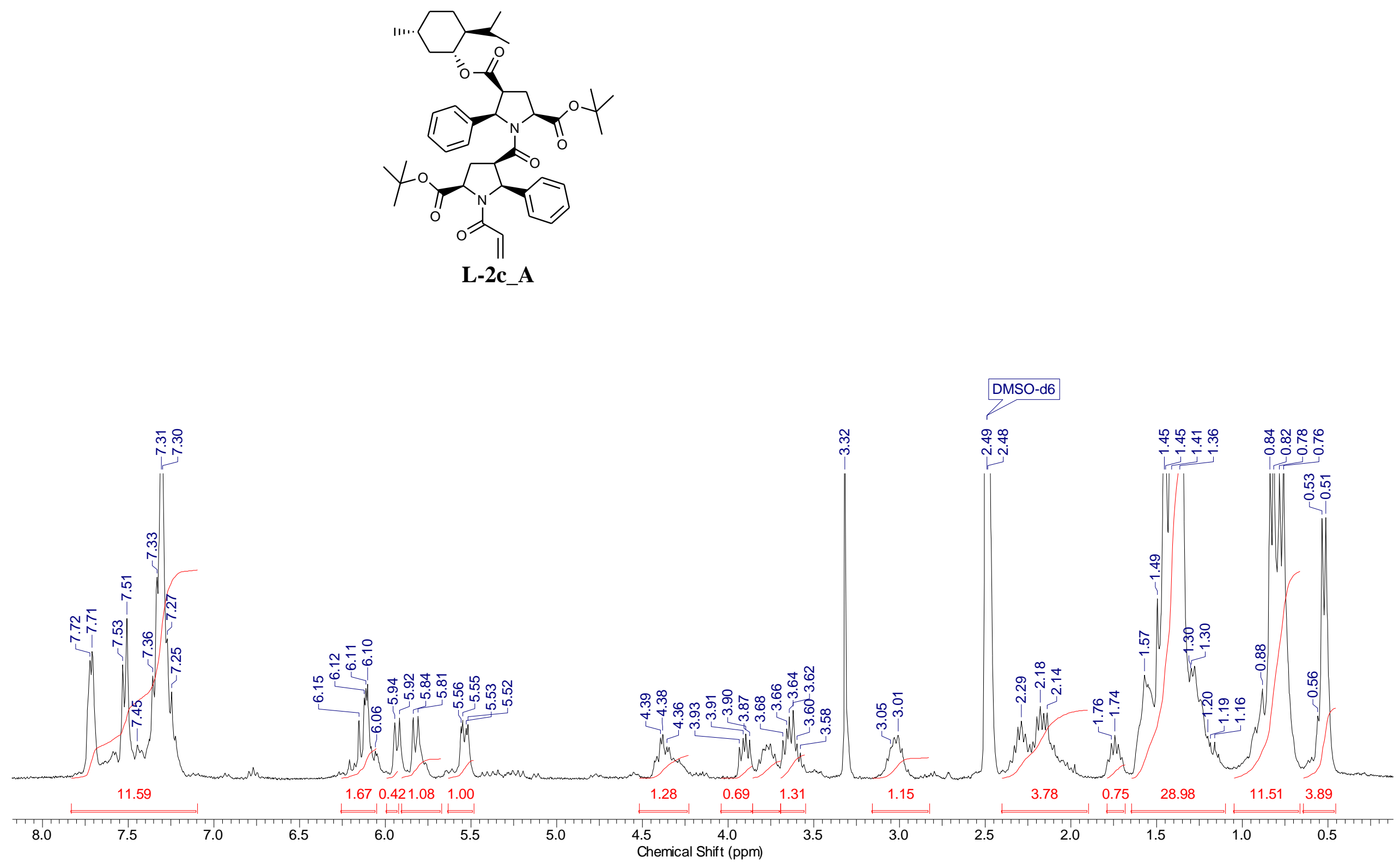


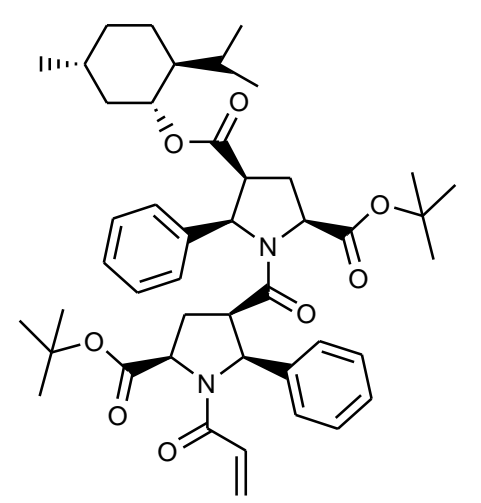

DMSO-d6

L-2c_A

ภㄷํㅇำ

लंग्लंक्ल

N

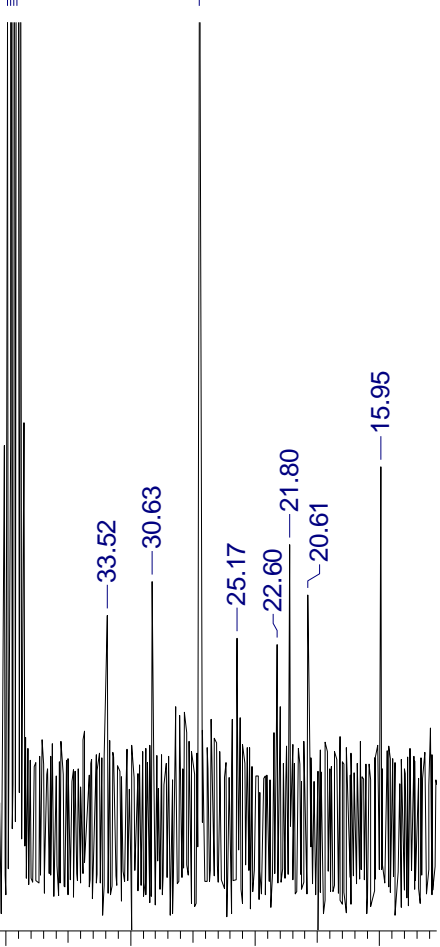

168

160

$\stackrel{\infty}{\stackrel{\sim}{\sim}}$

กิ

กิ

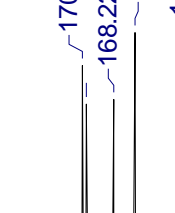

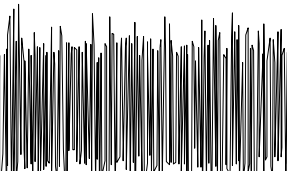

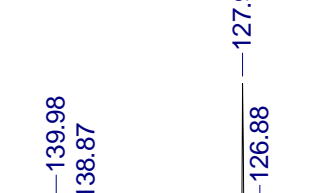

in.

136
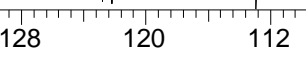

$\begin{array}{lll}104 & 96 & 88\end{array}$ 


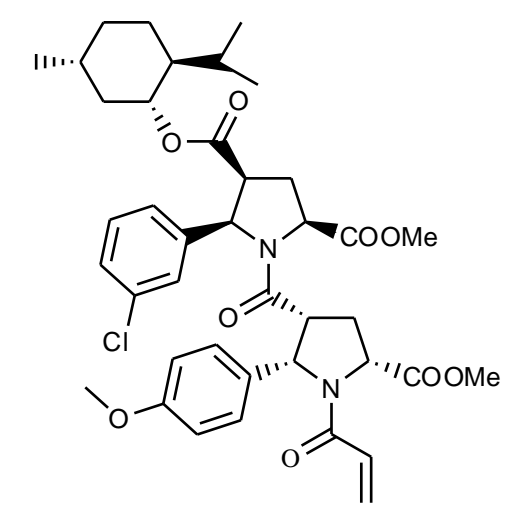

L-2d_A

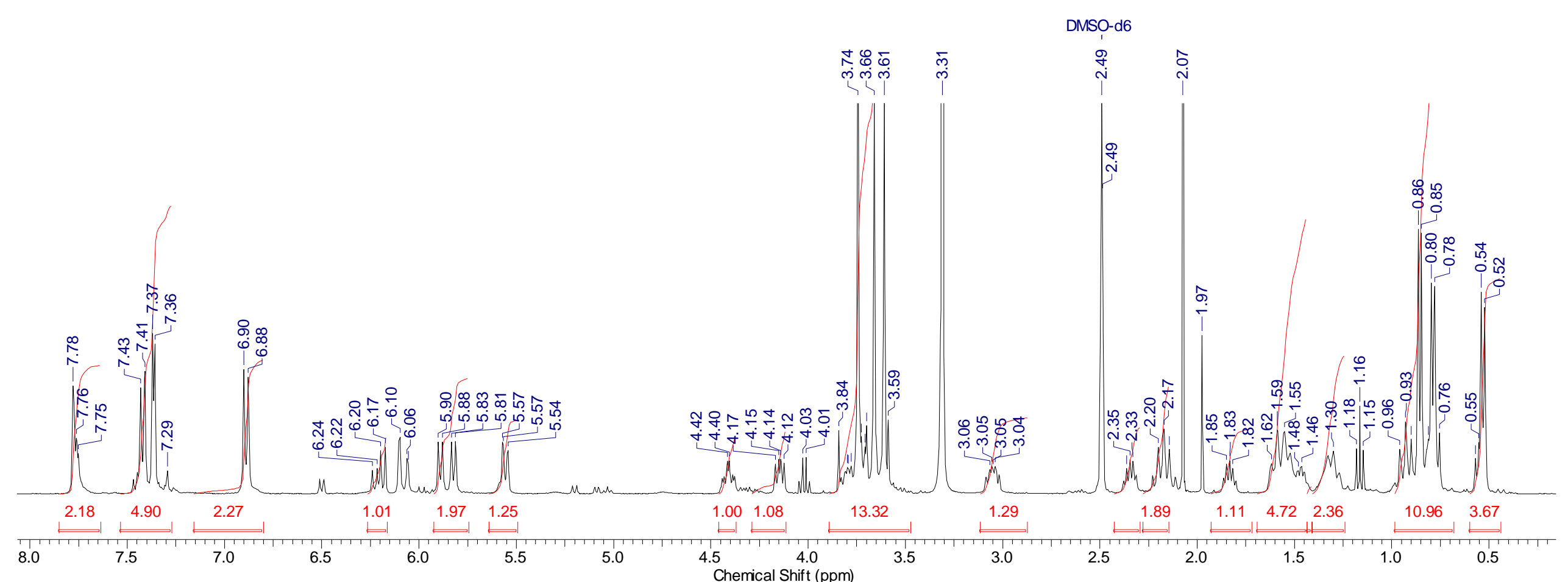



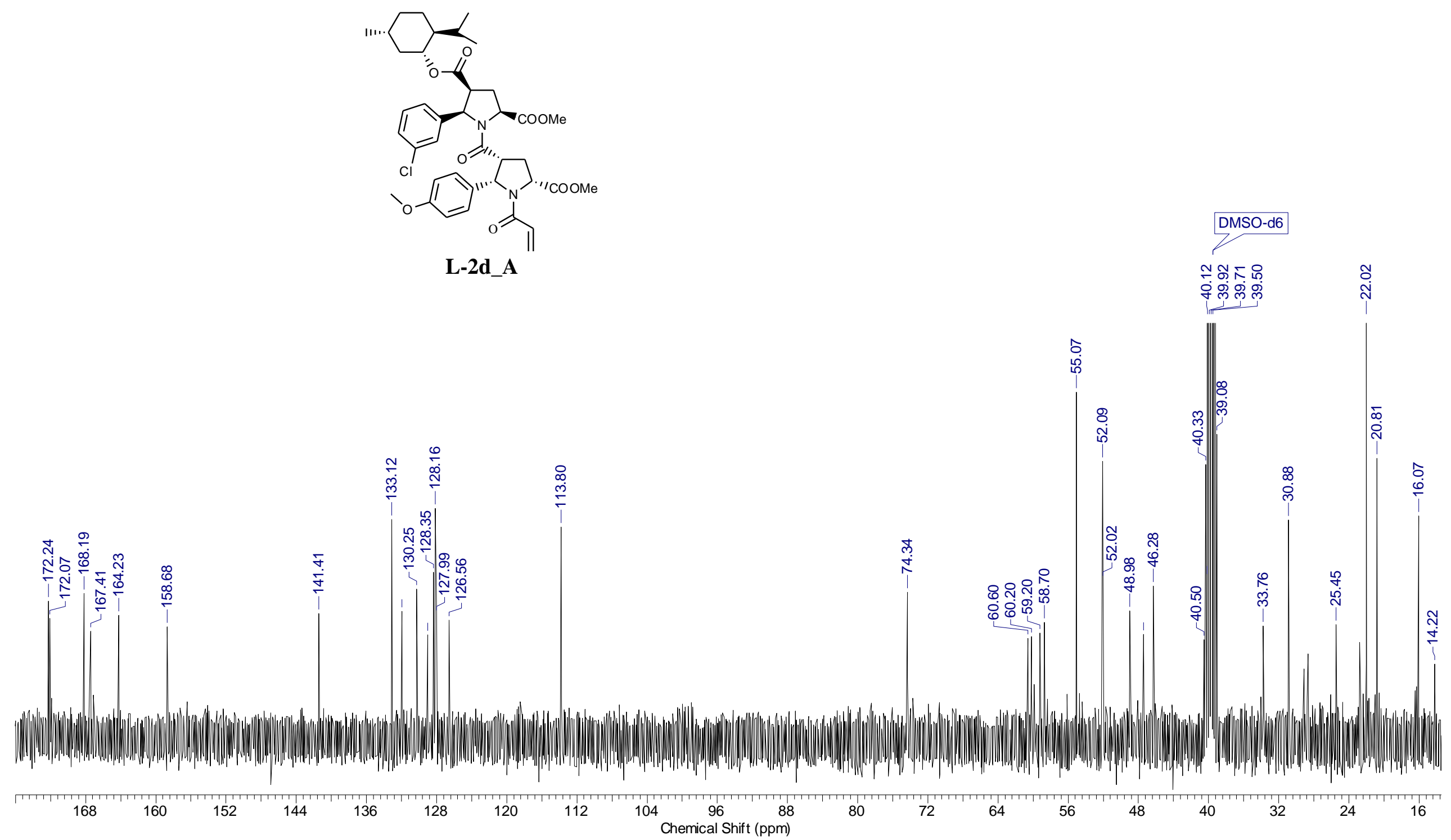


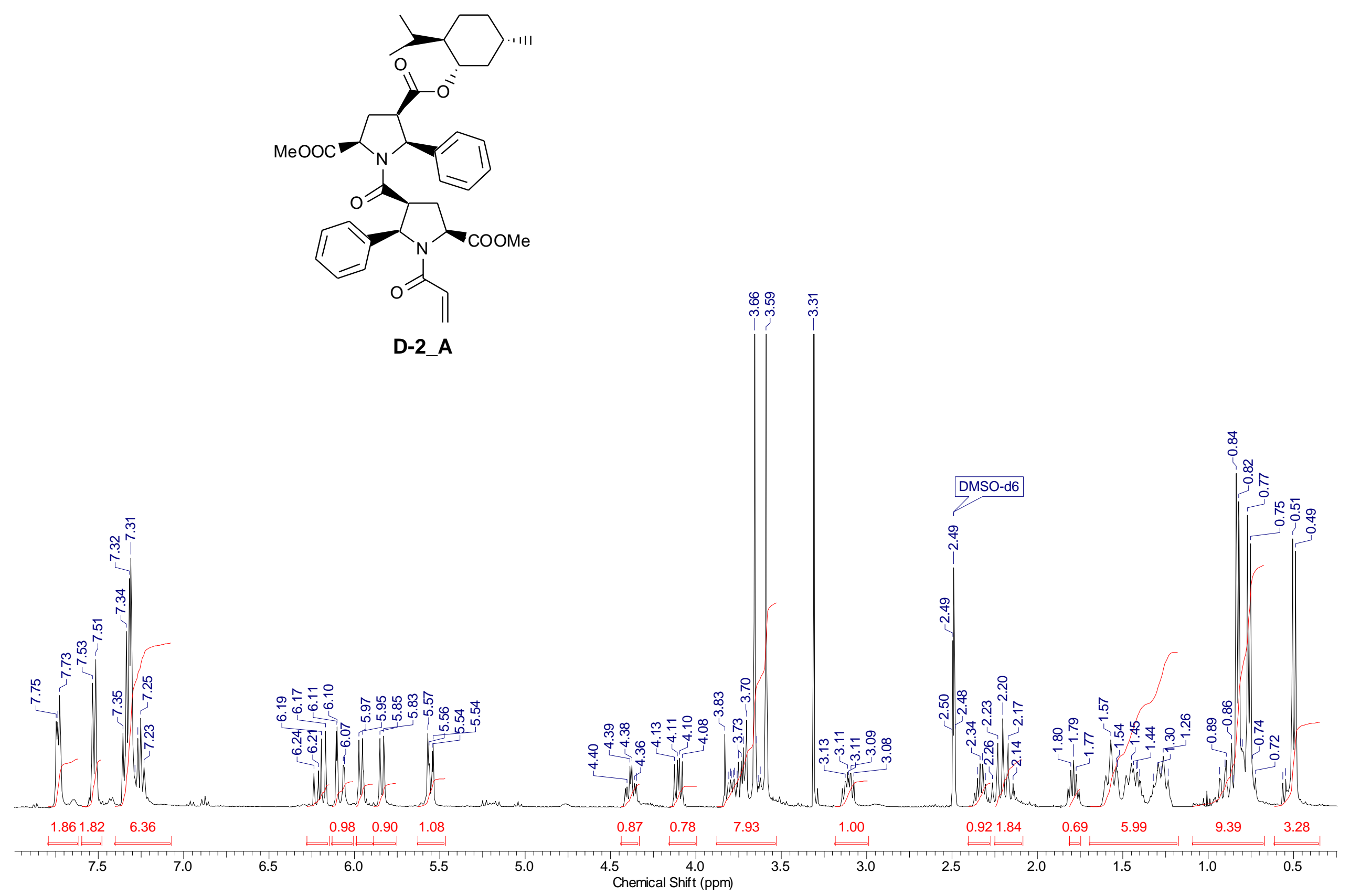



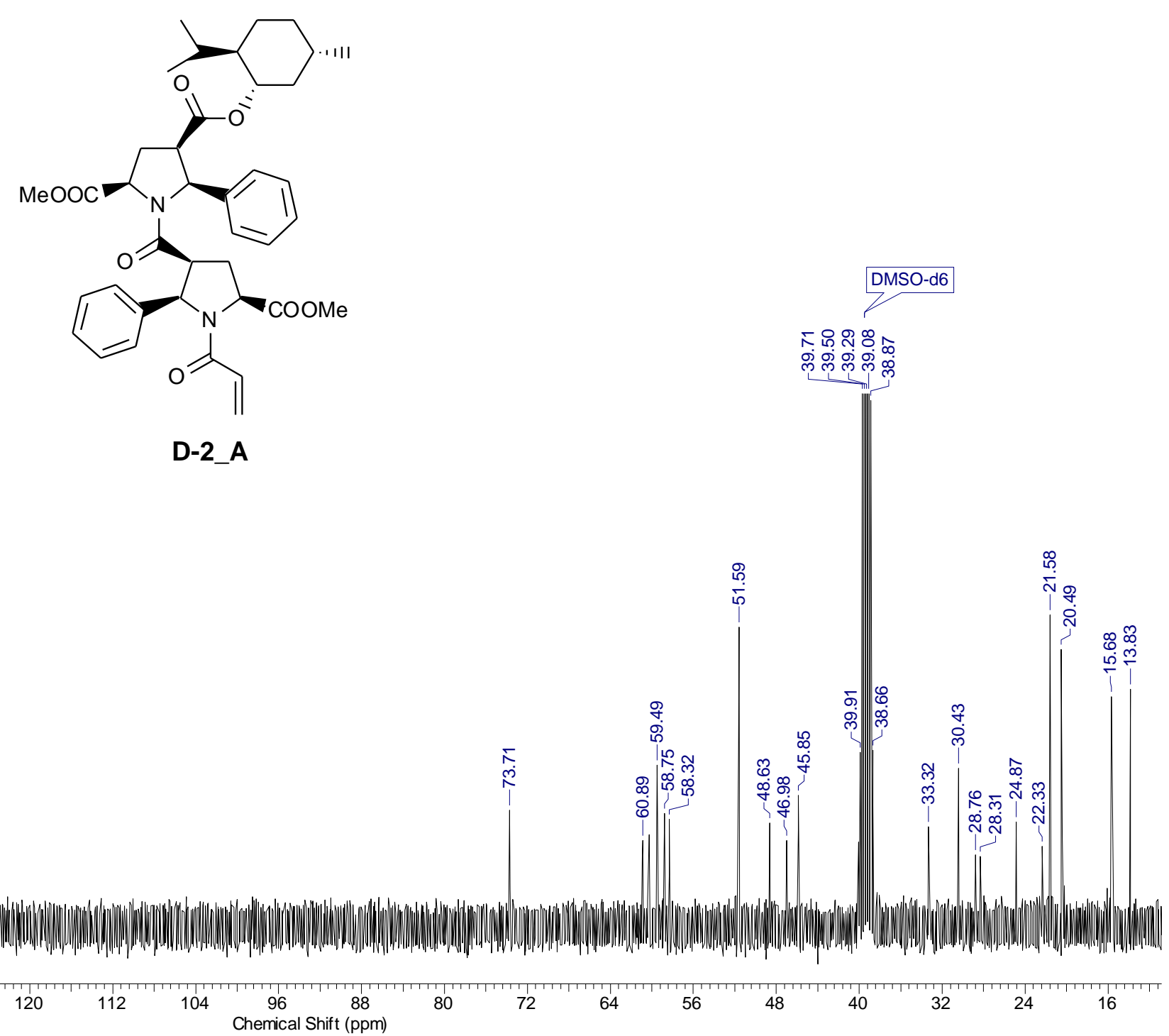


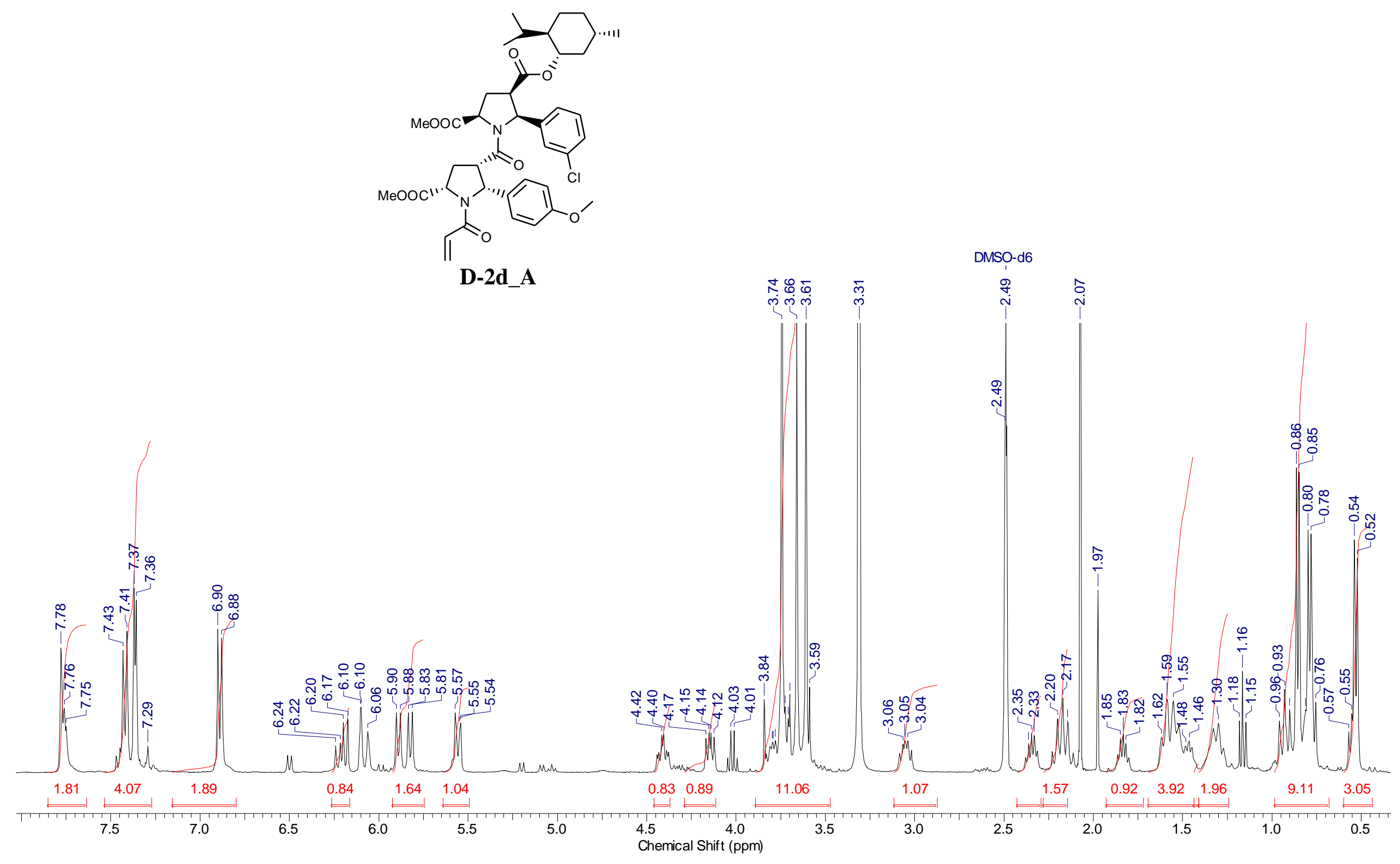




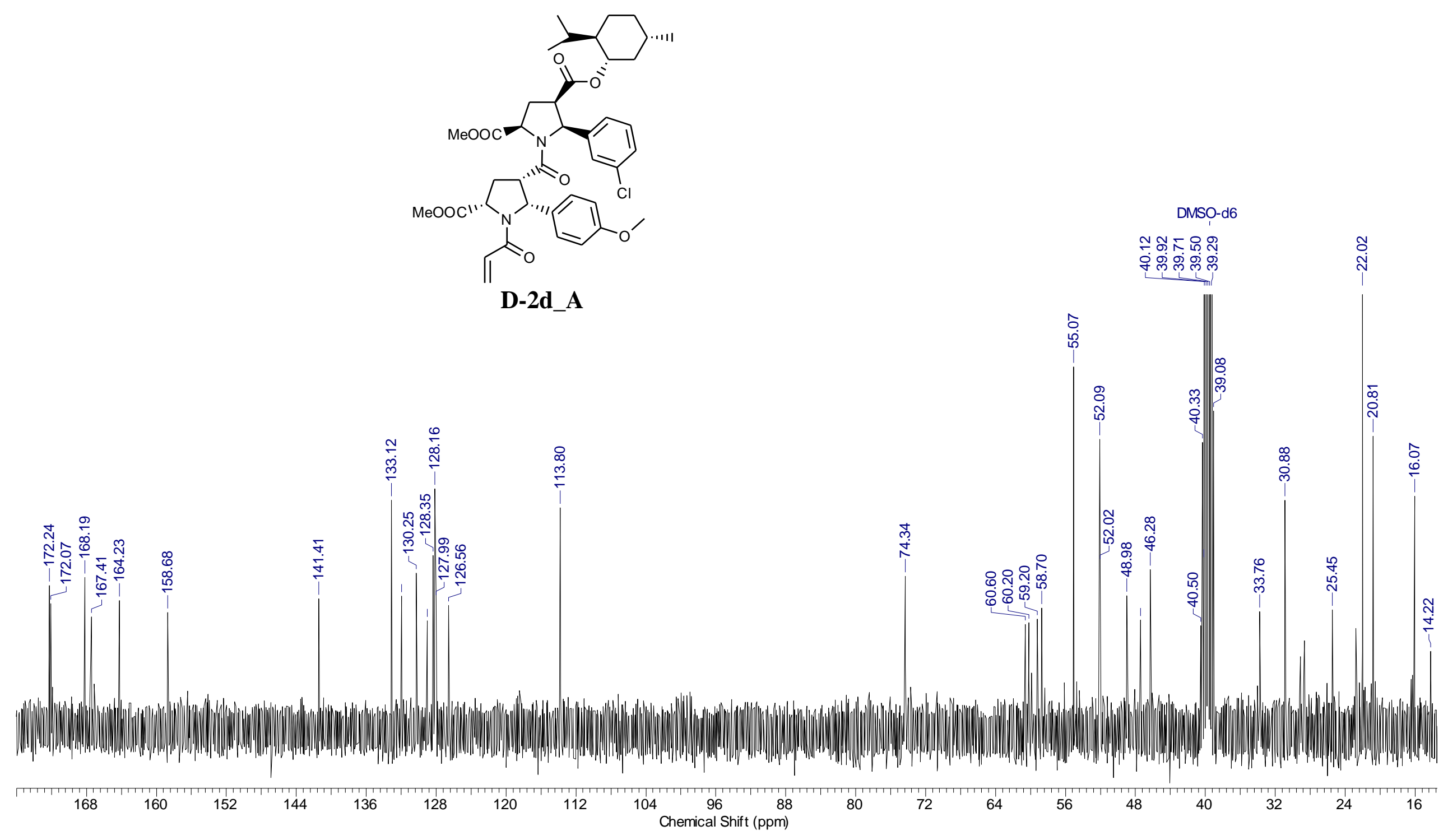



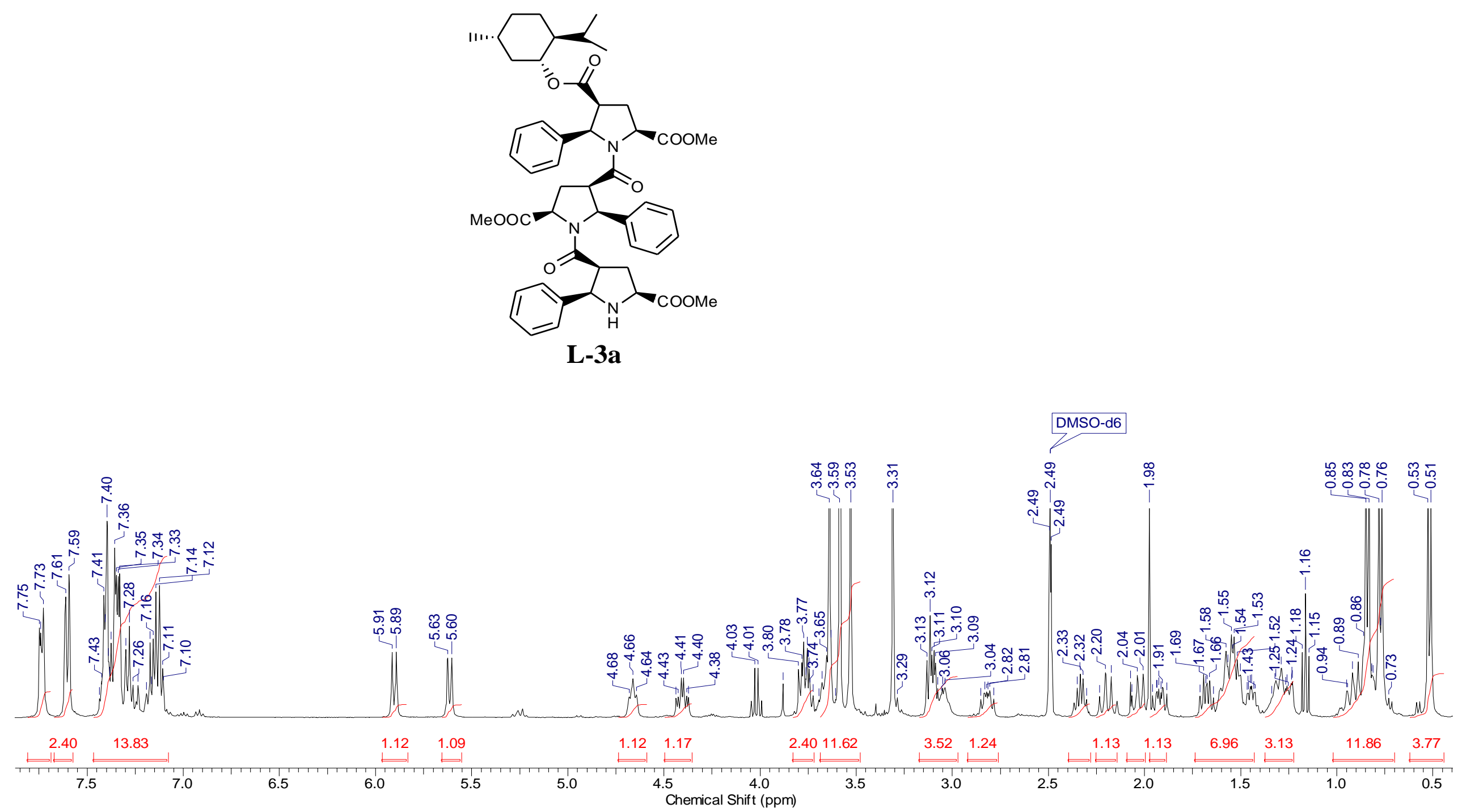

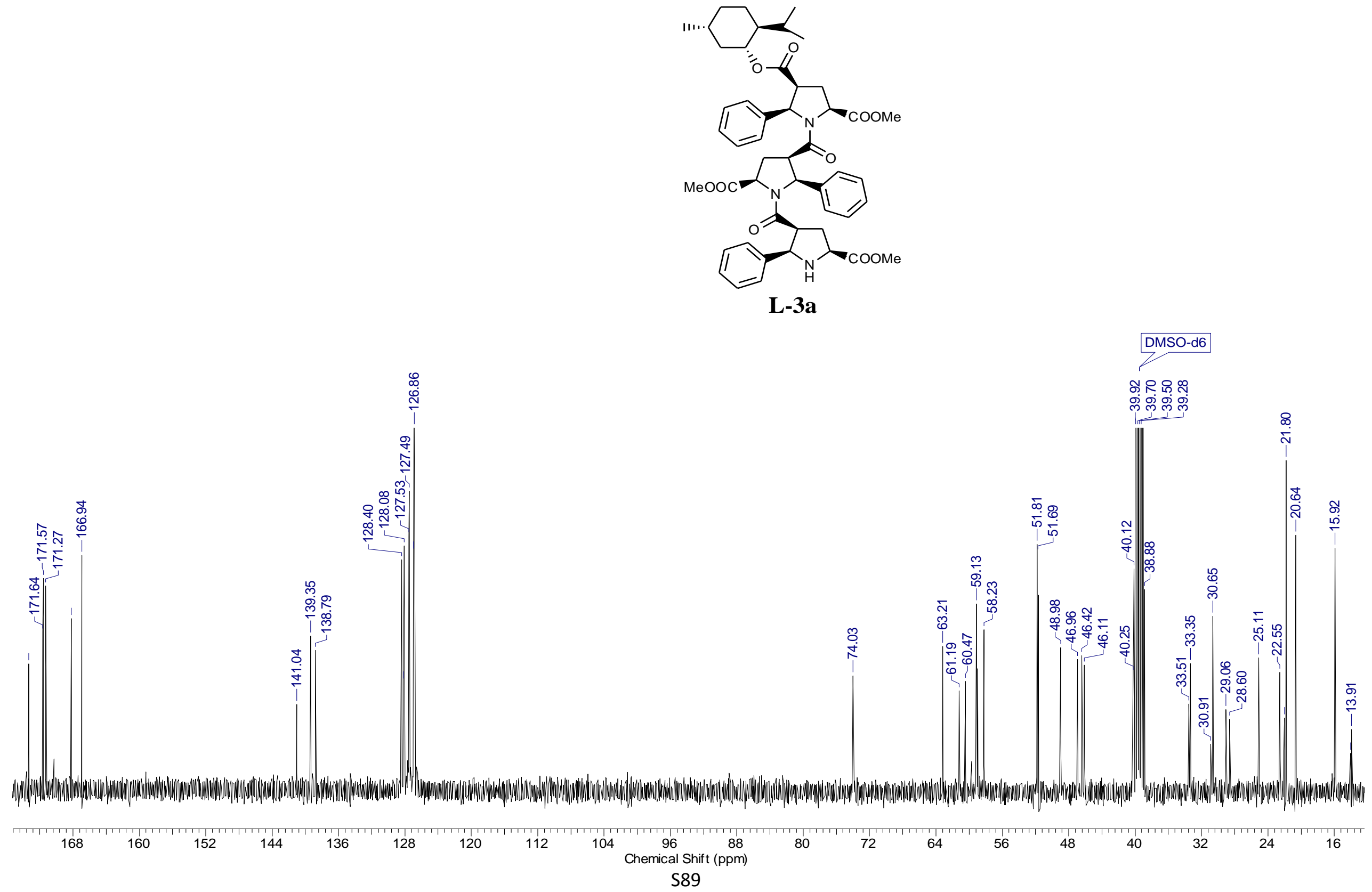


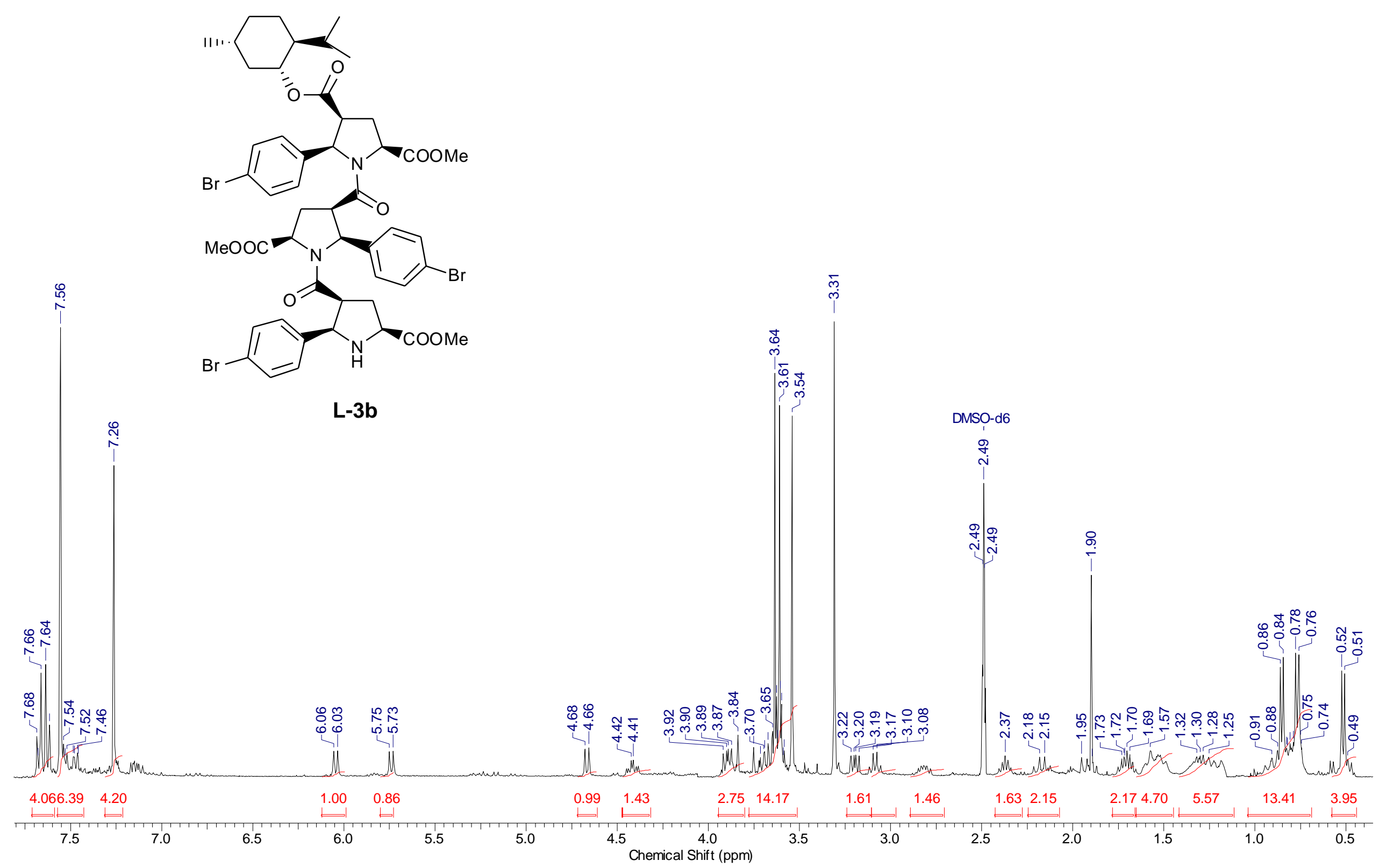



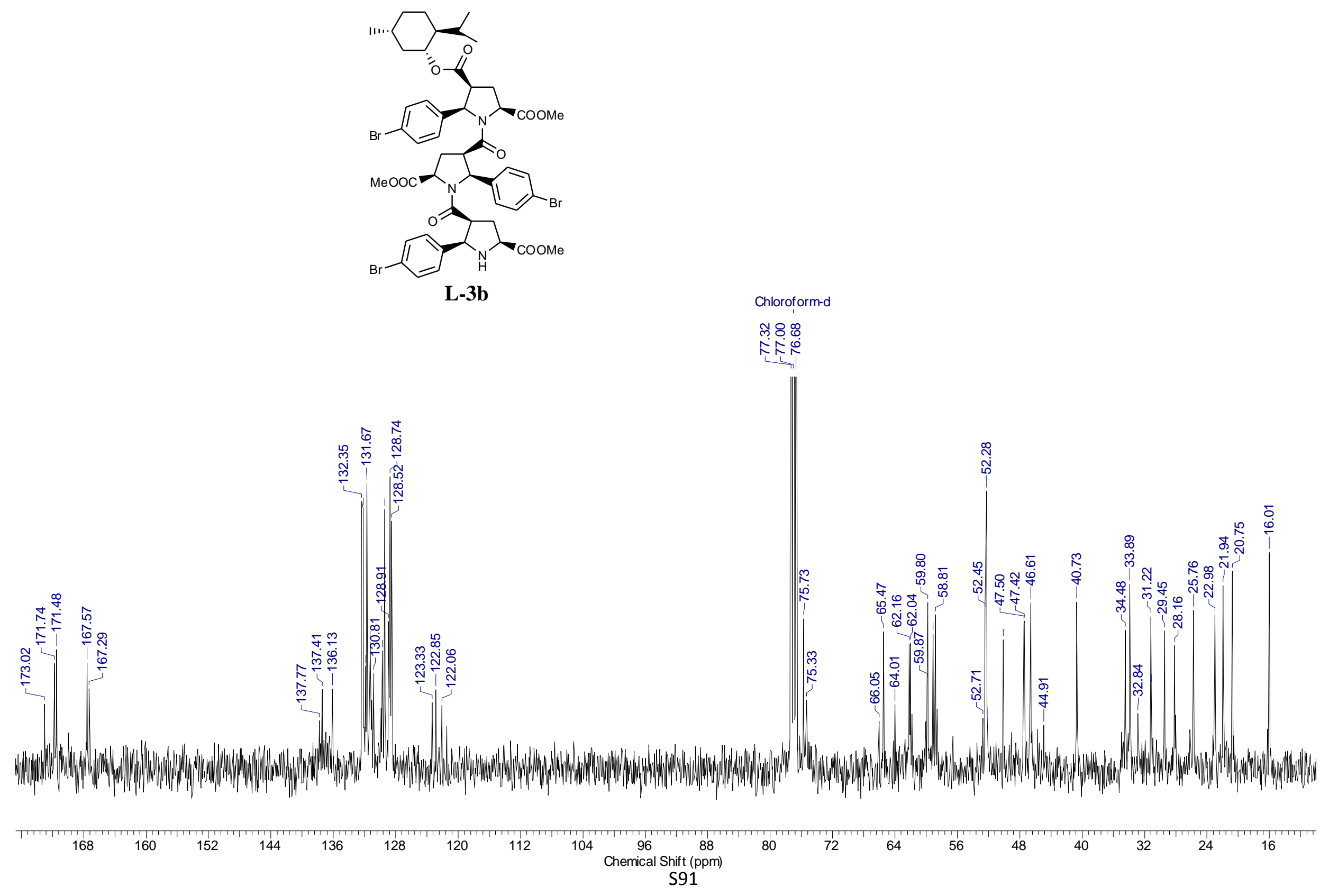

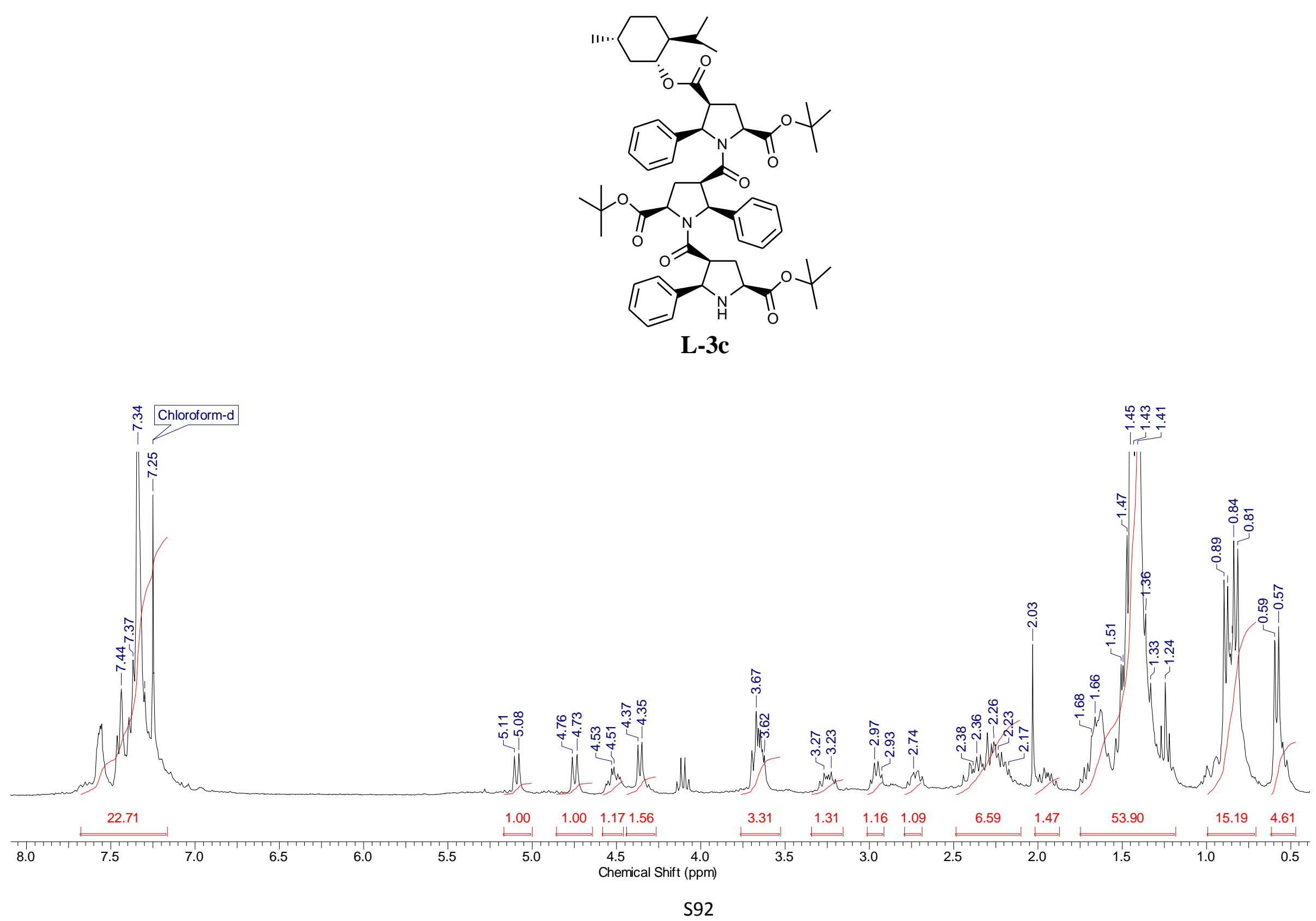

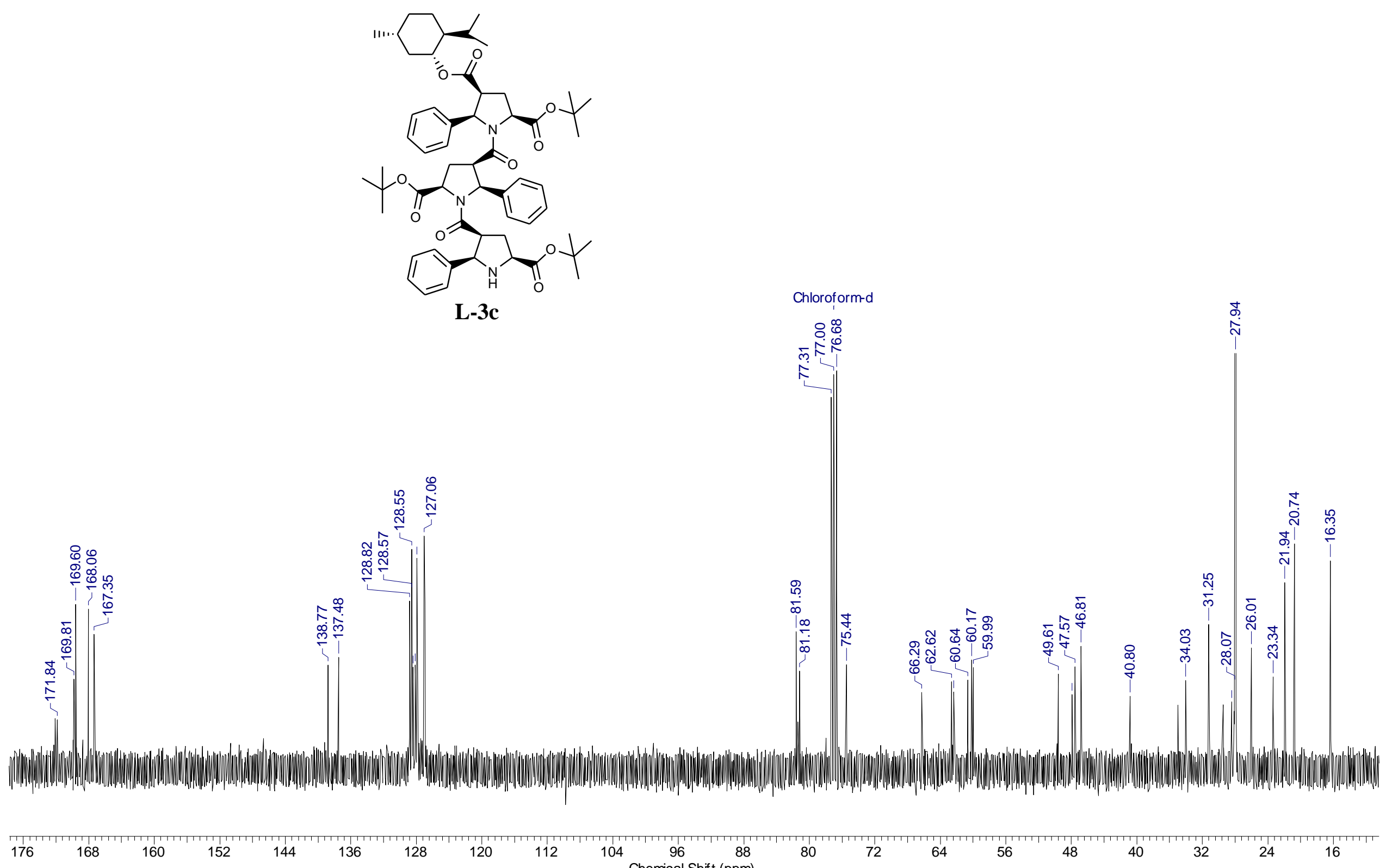

136 128 120 $12 \quad 104 \quad 96 \quad 88$ 80 72 64 48 40 24 


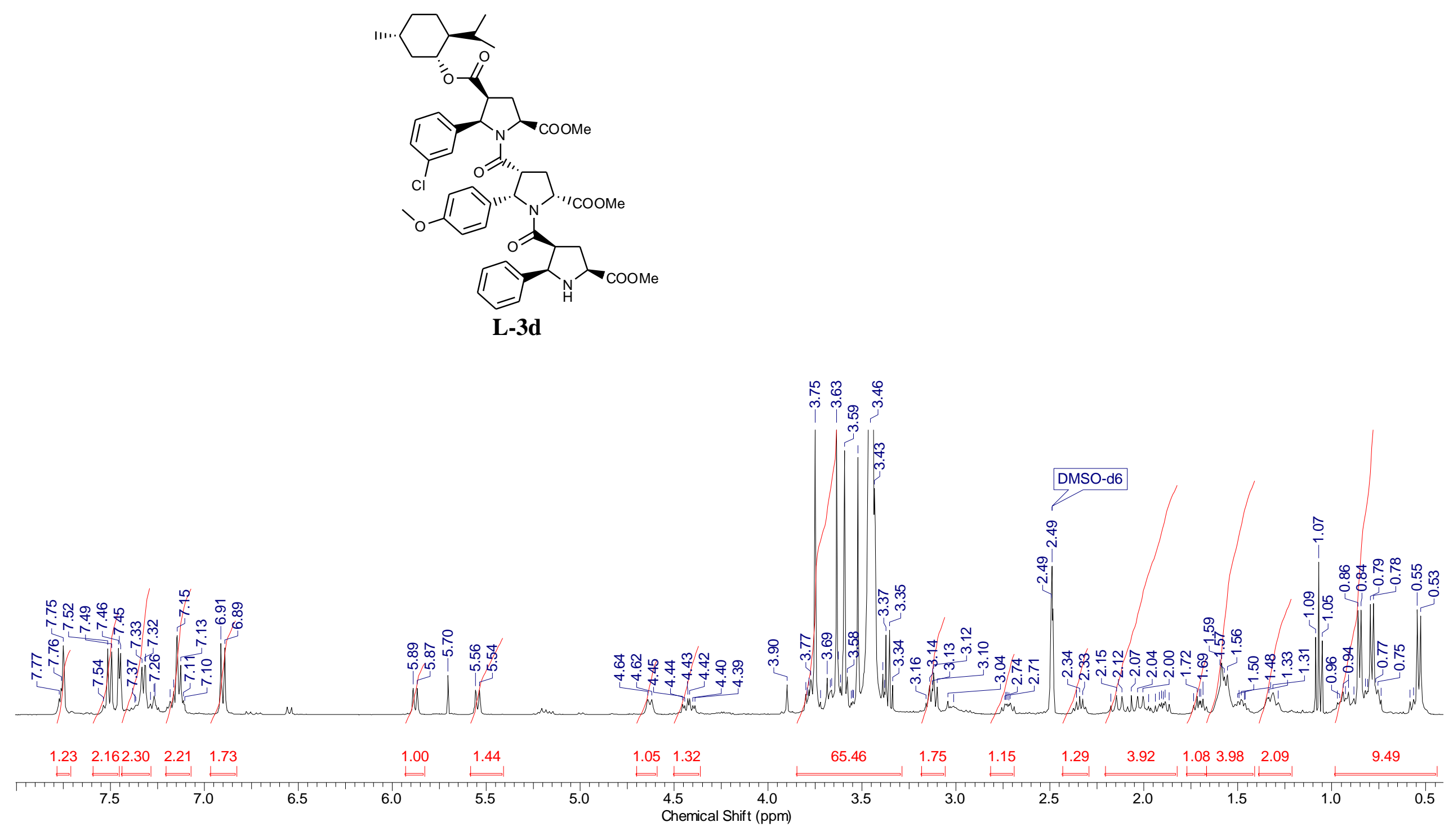




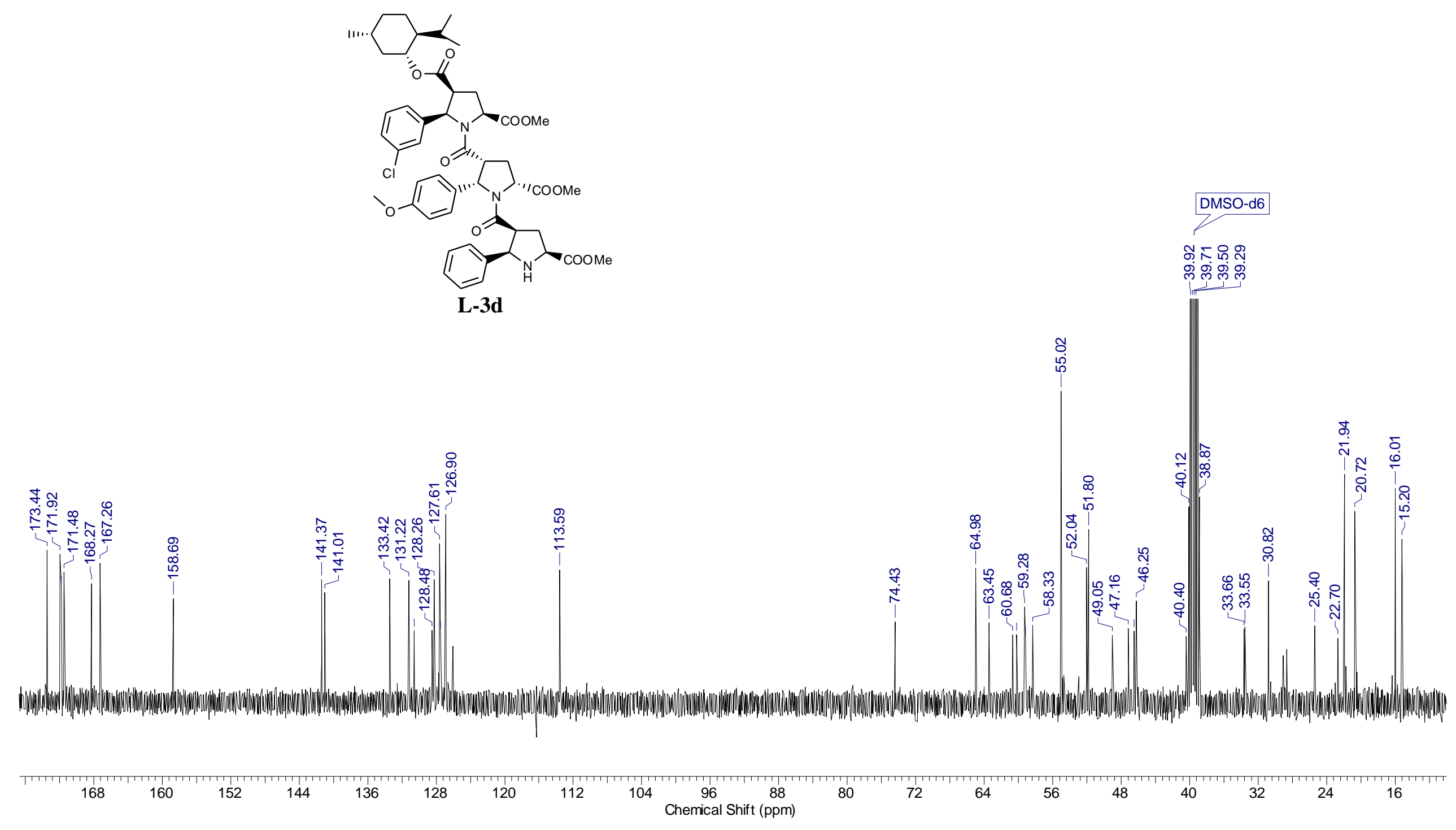



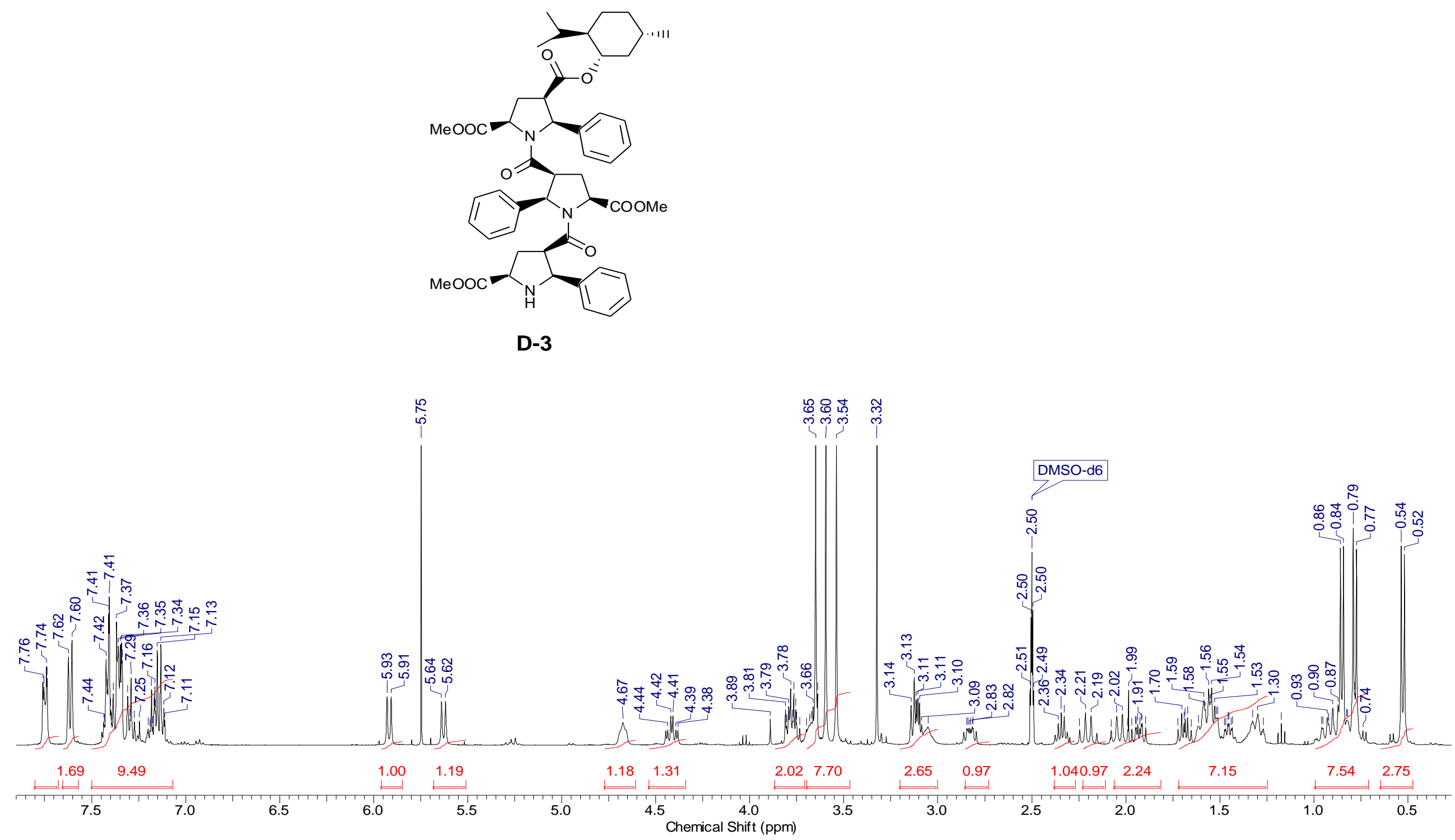

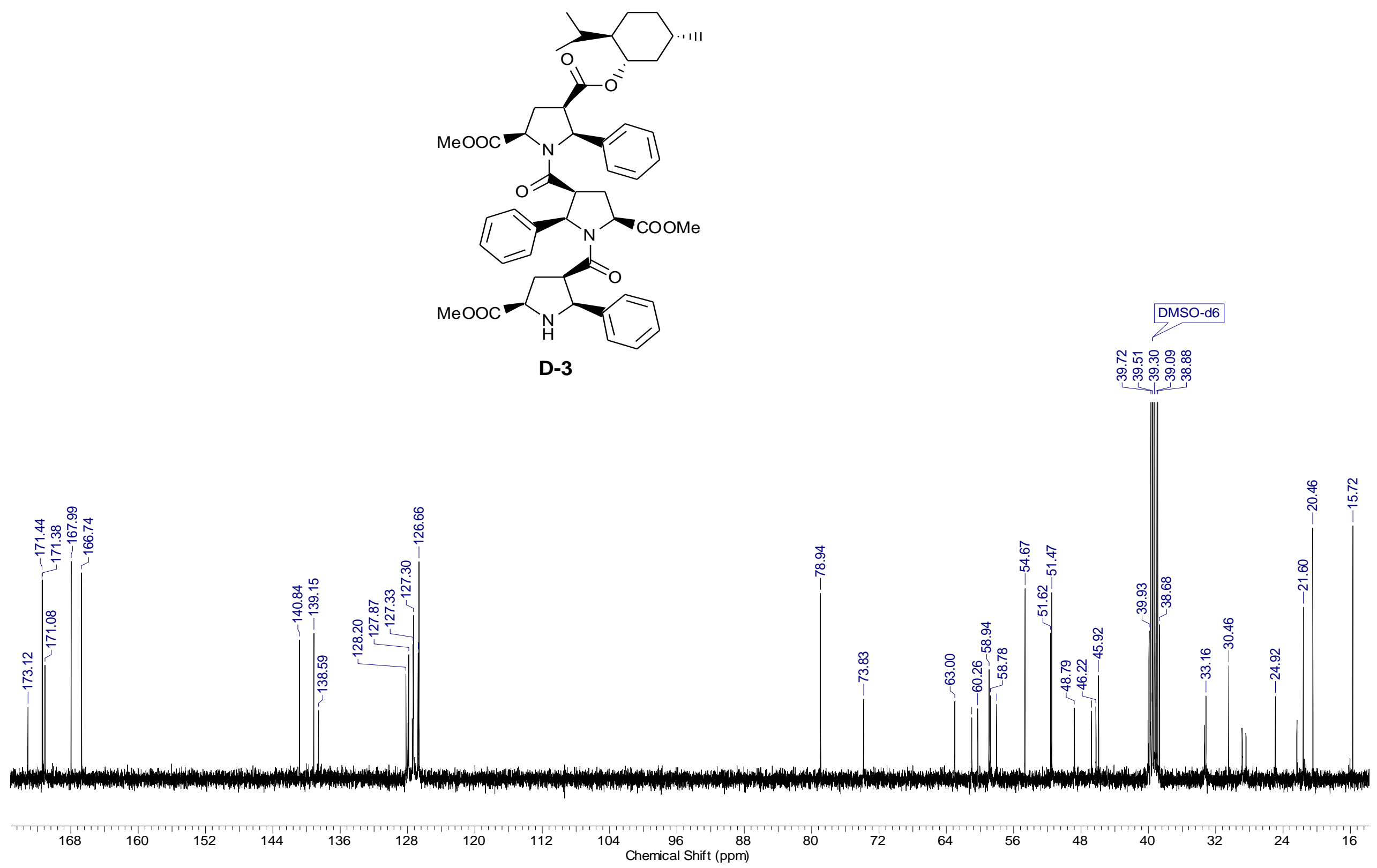


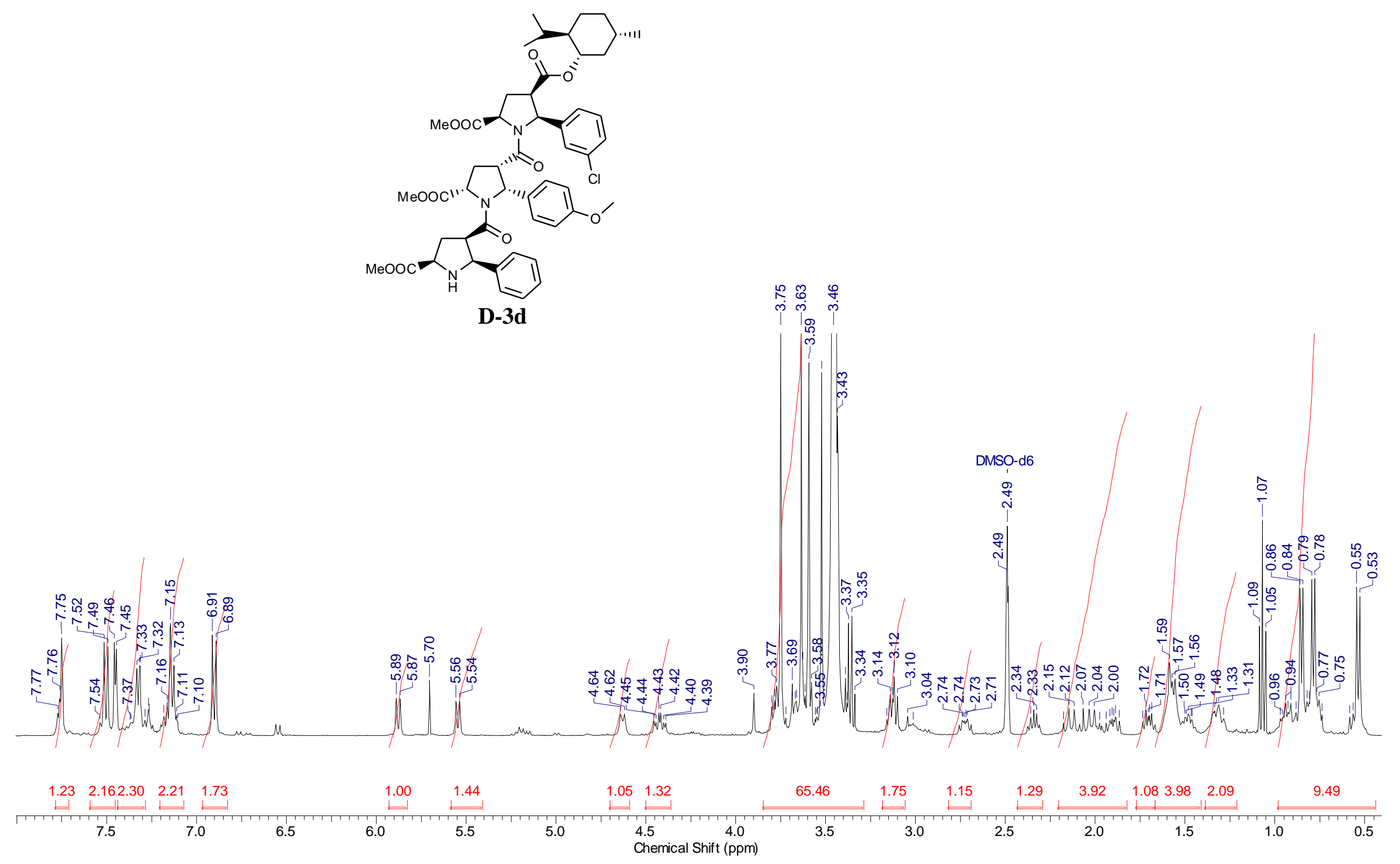



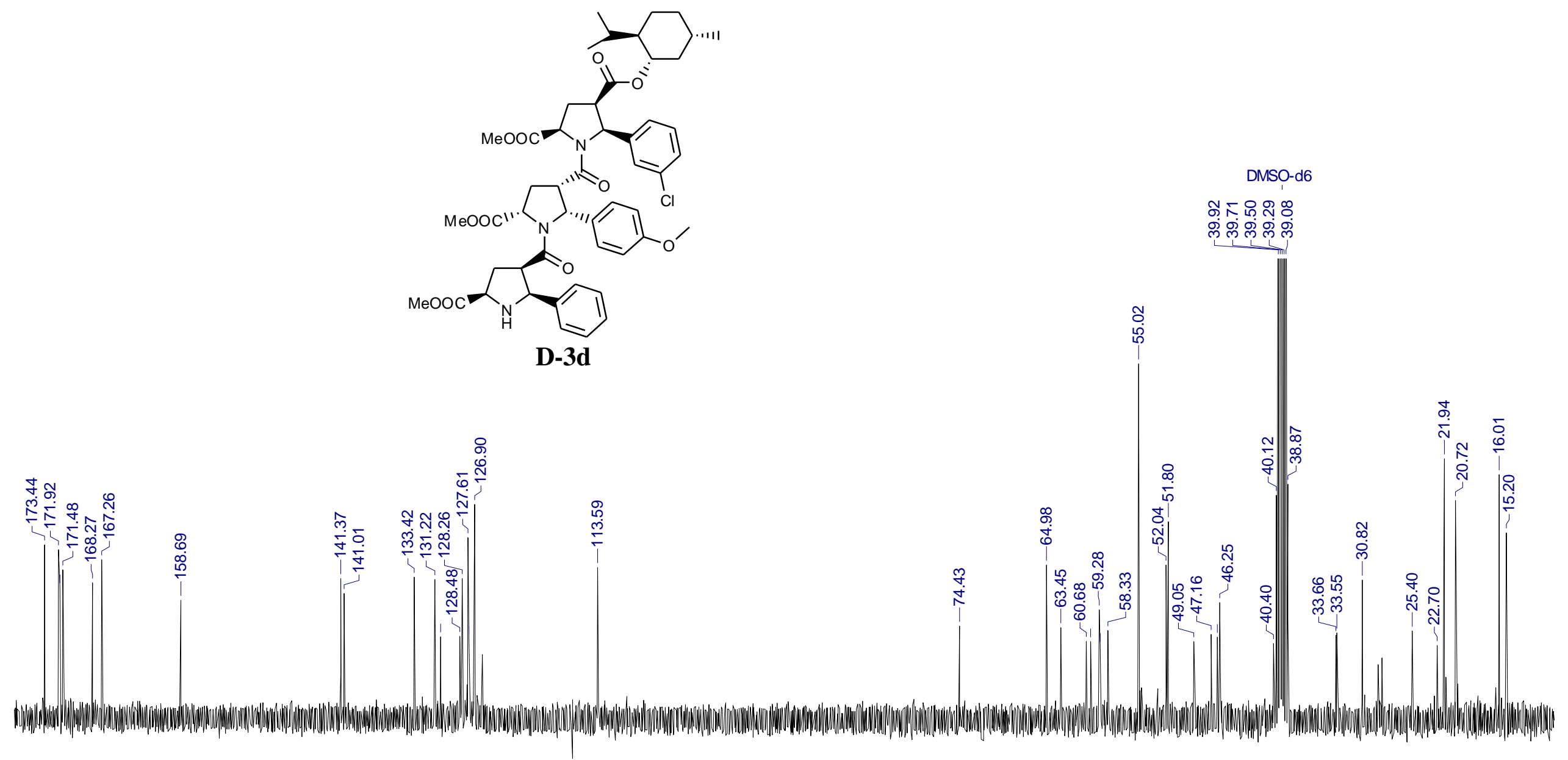

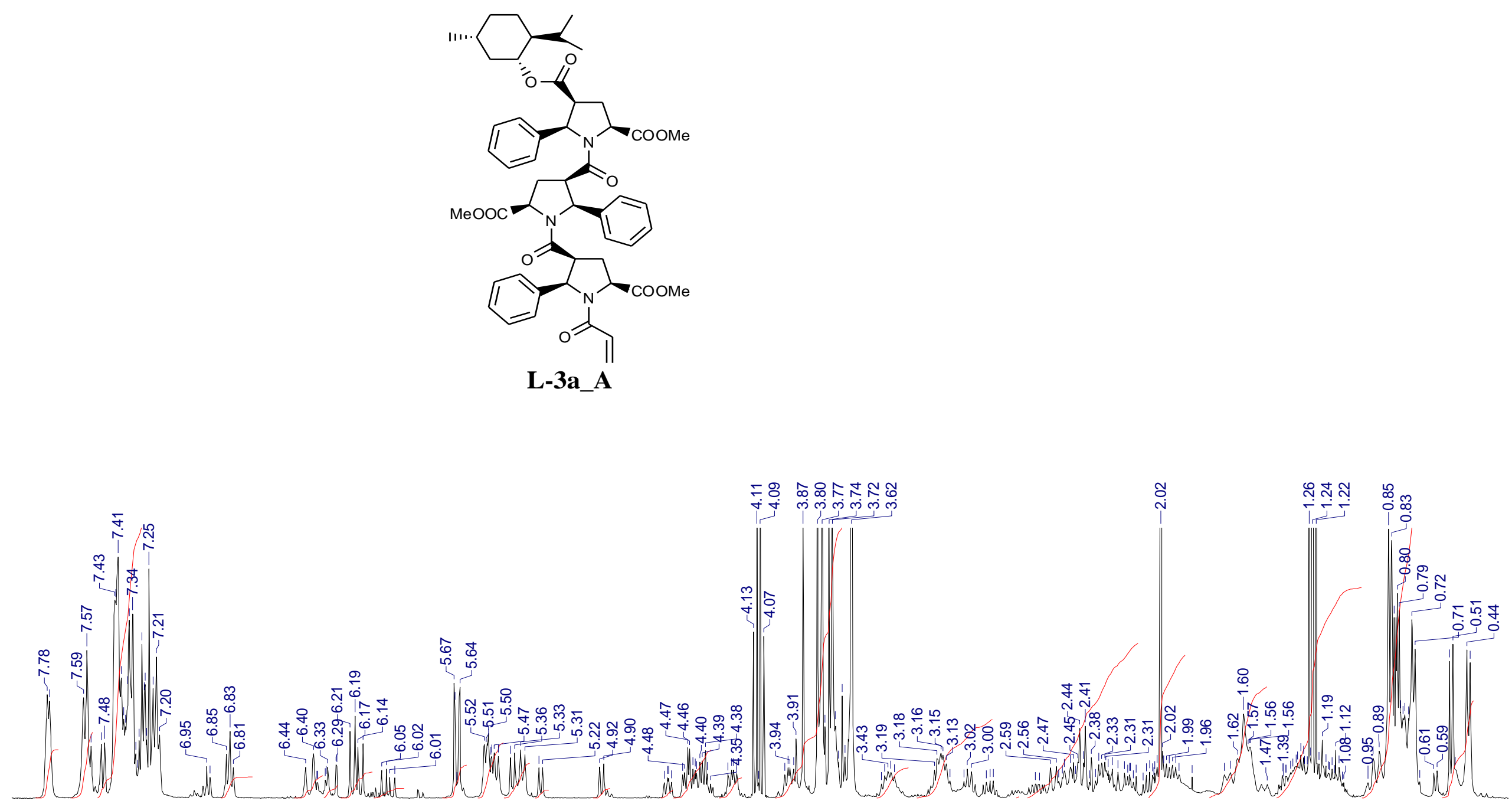

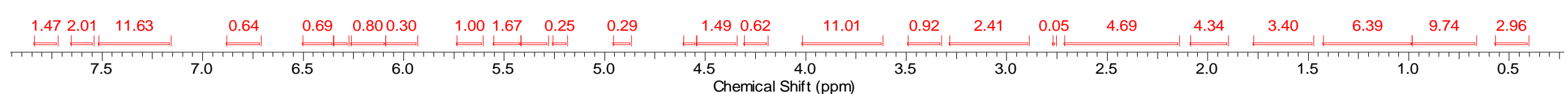




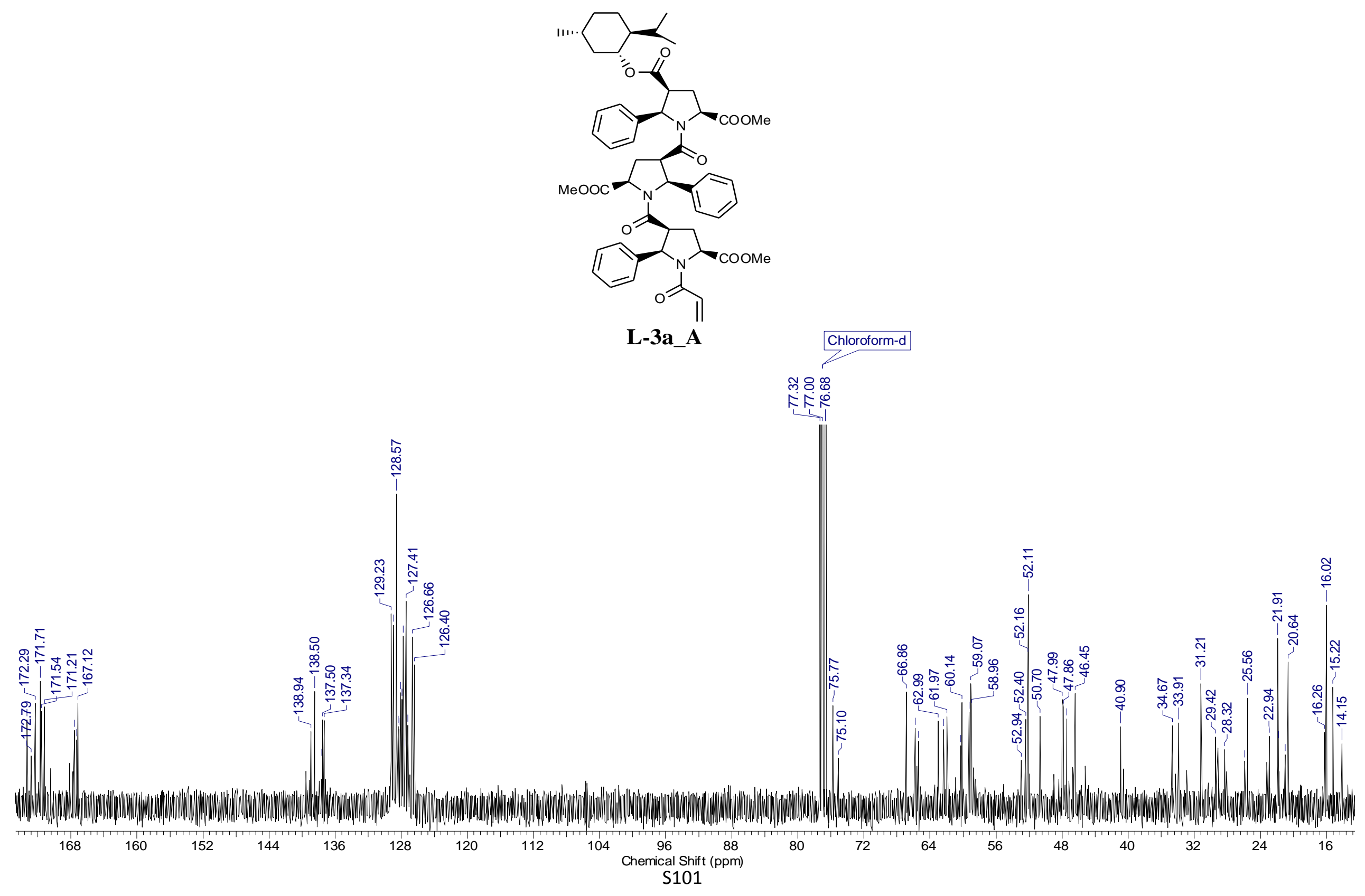




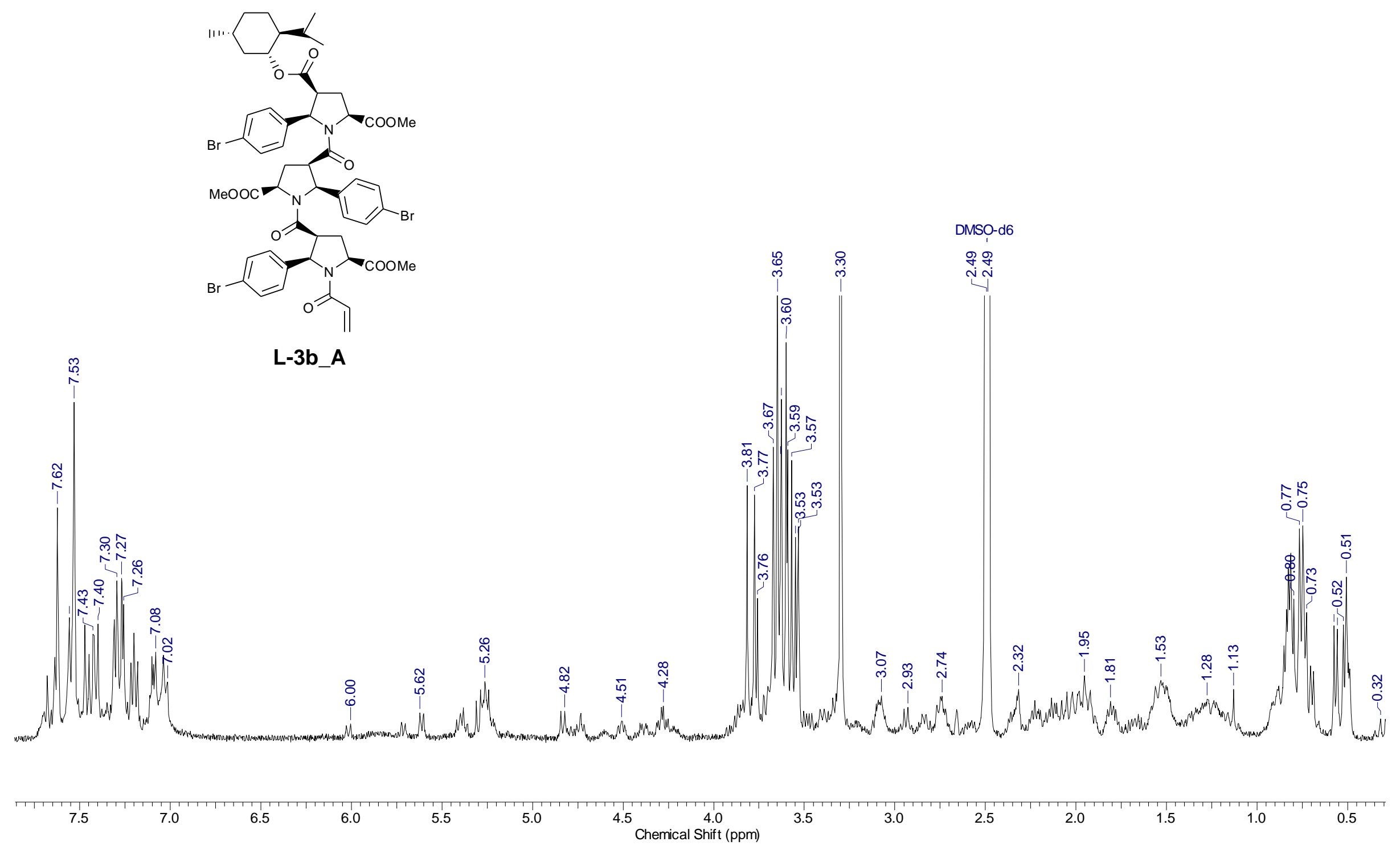




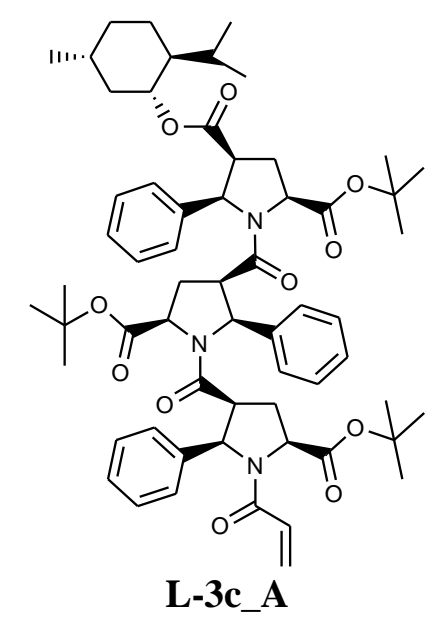

DMSO-d6

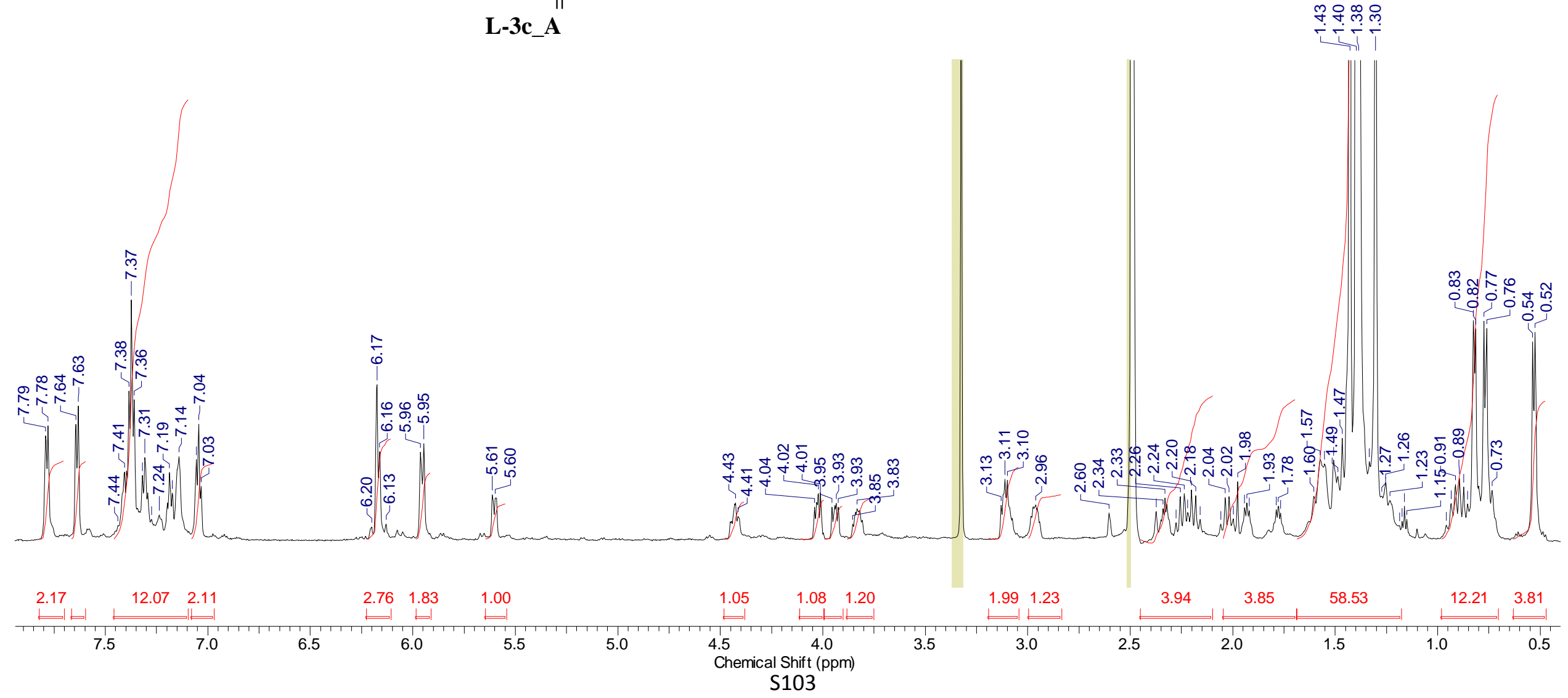




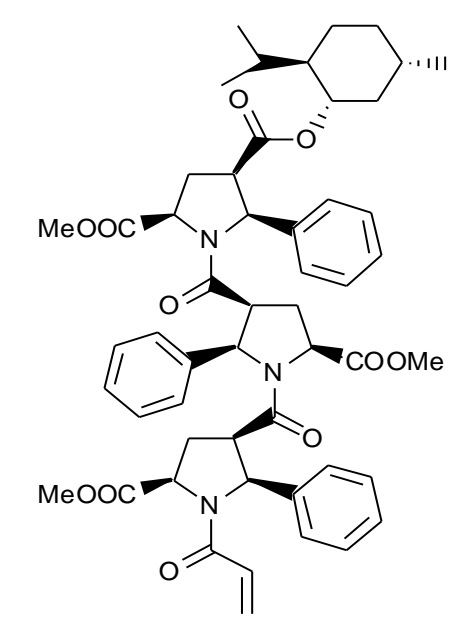

D-3_A

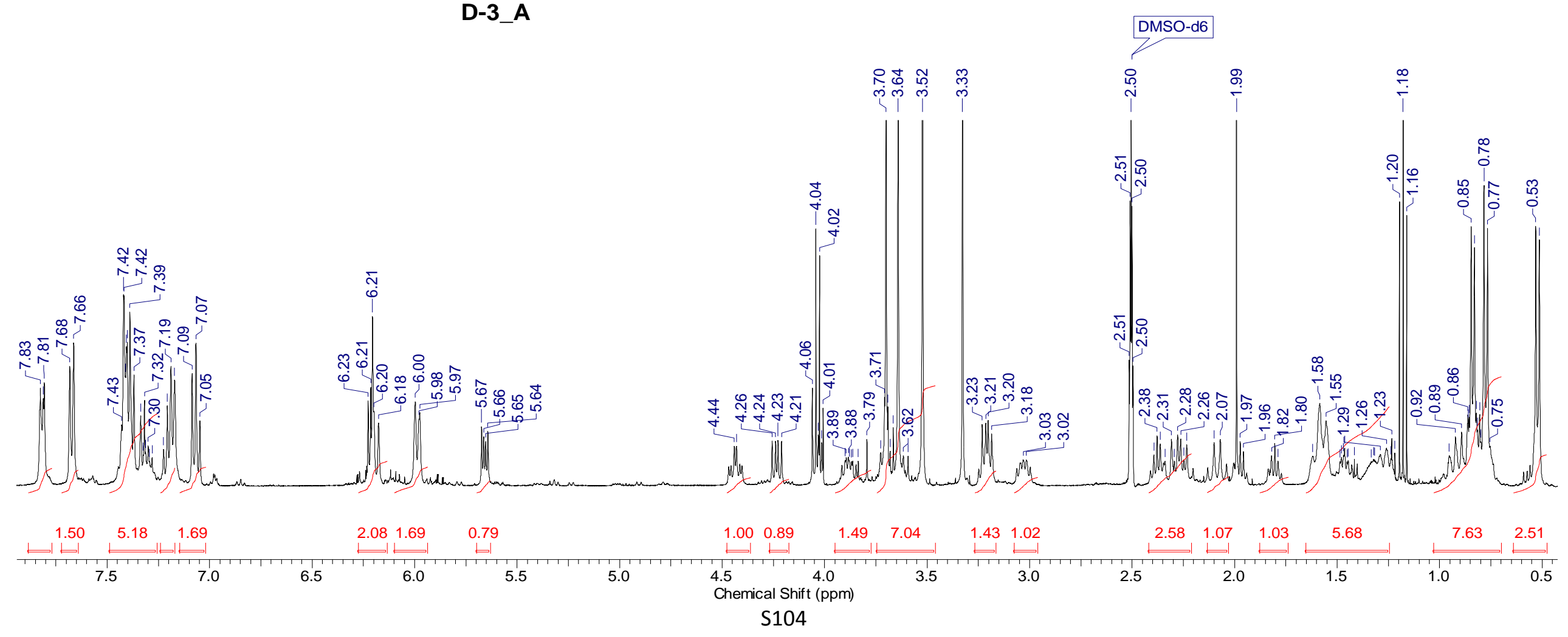




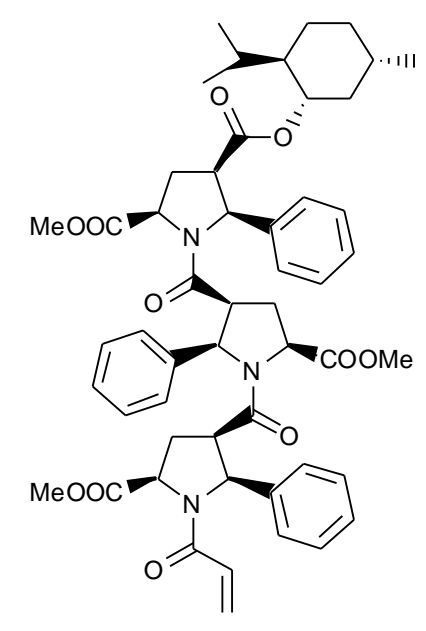

D-3_A

क्लिंल क्ल

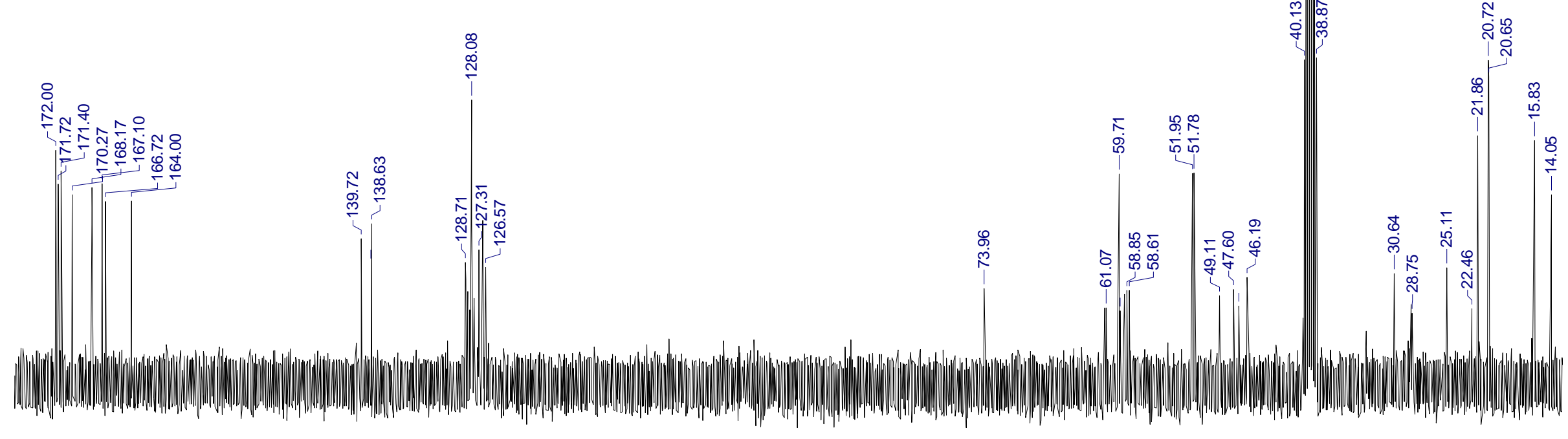

168 160 152144136 128 120 12 $04 \quad 96$ 

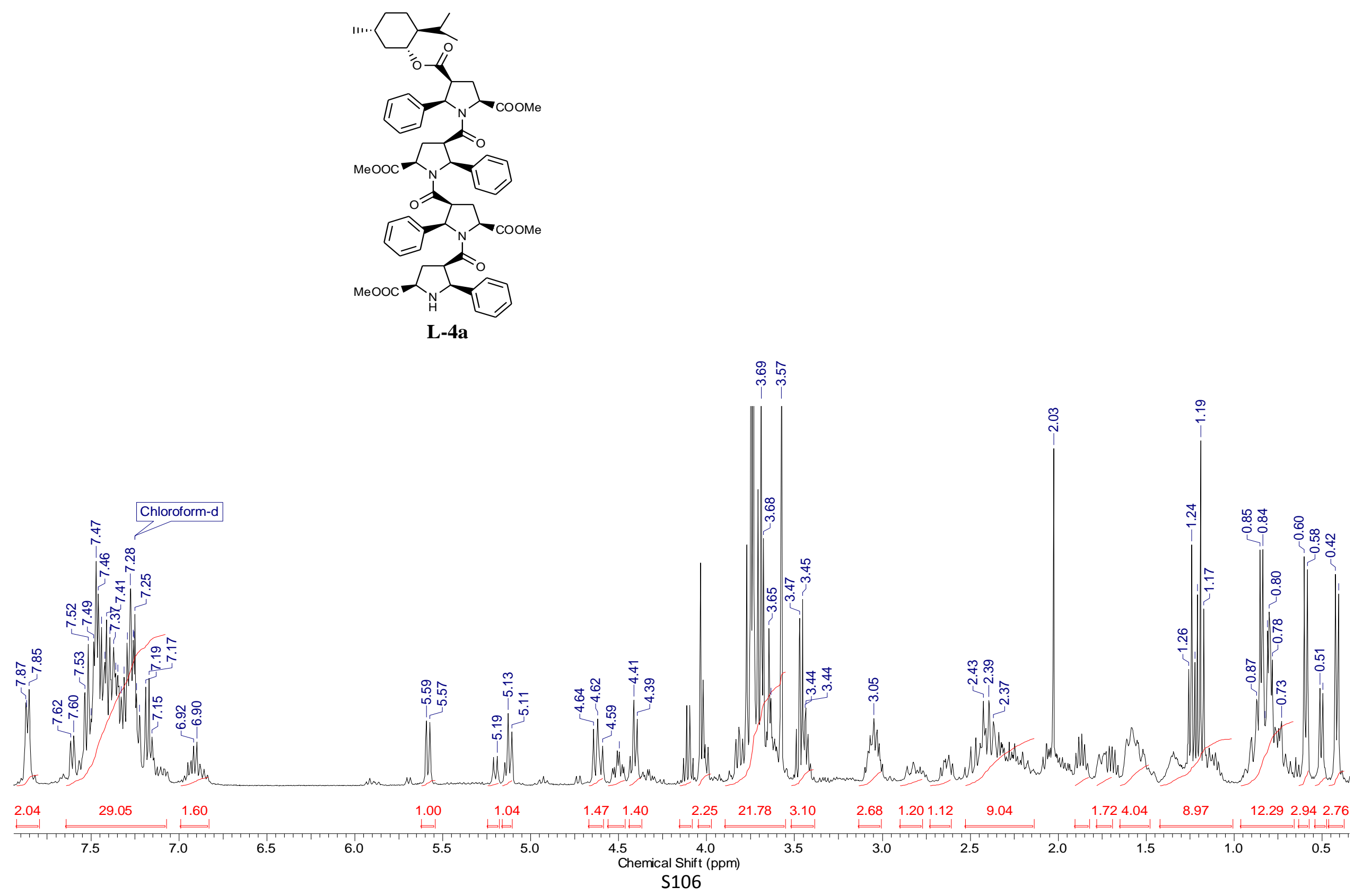


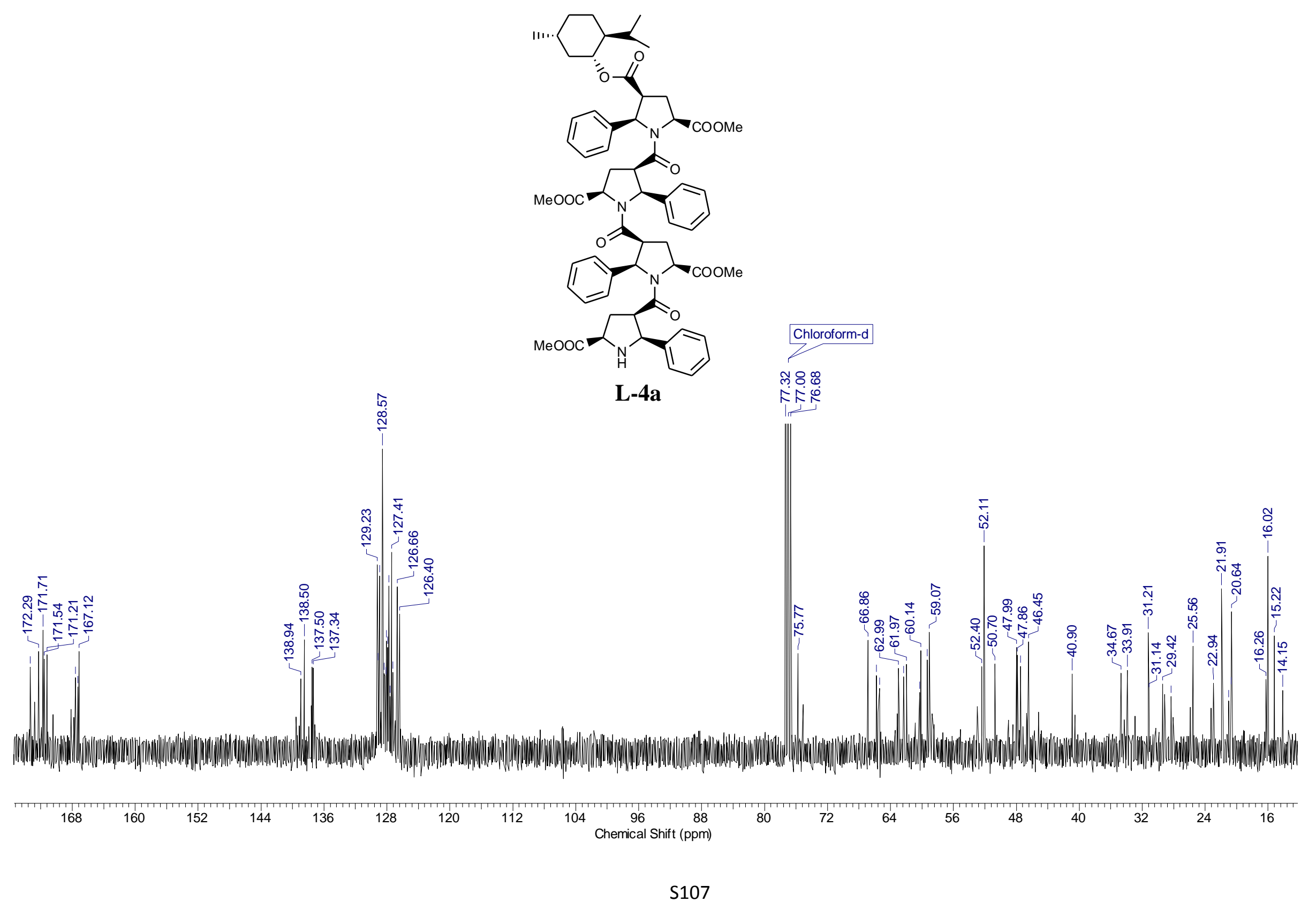




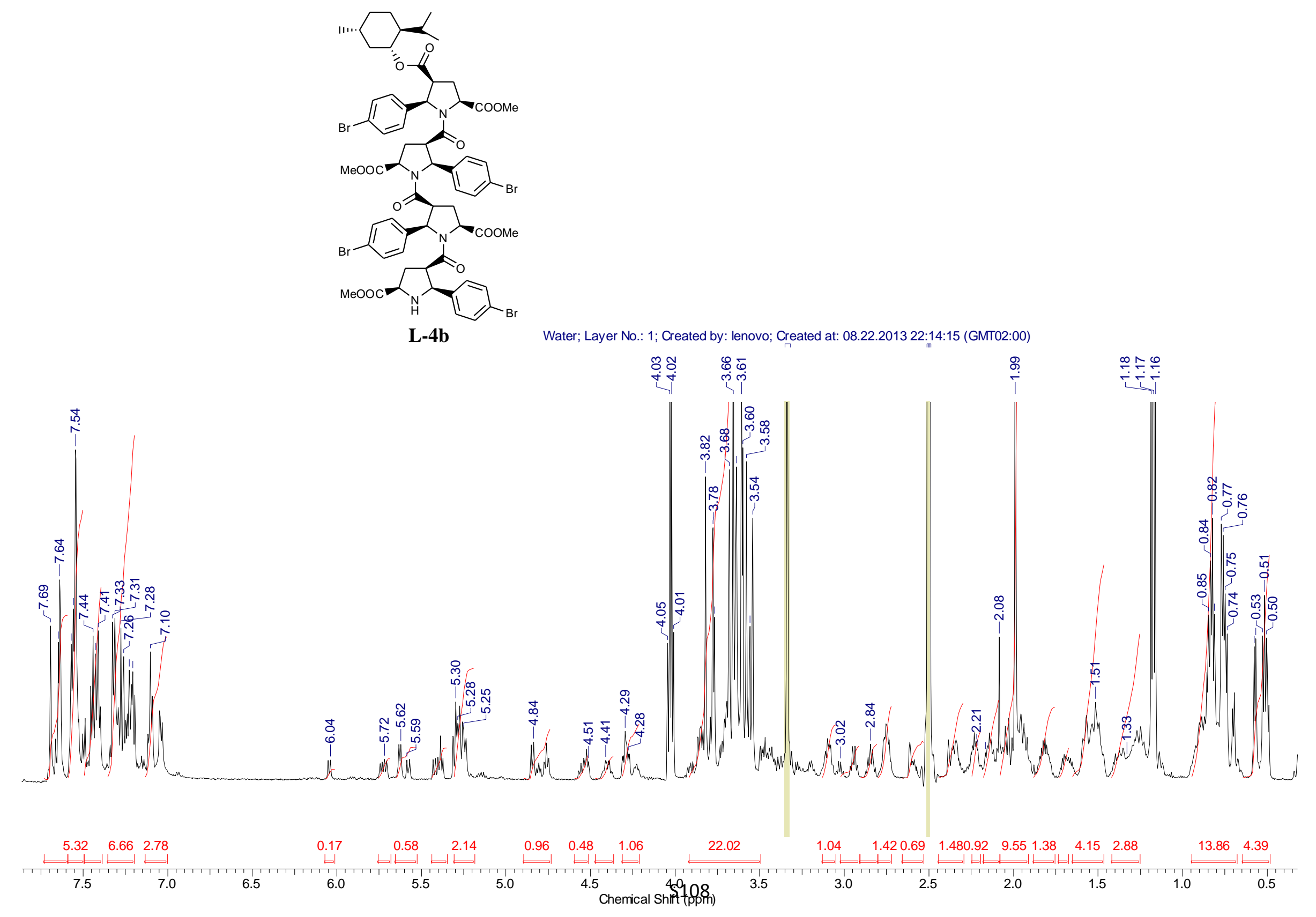




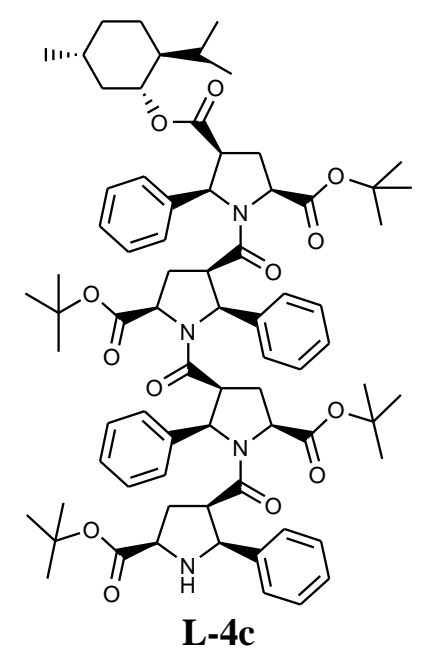

DMSO; Layer No.: 1; Created by: lenovo; Created at: 08.22.2013 22:22:54 (GMT02:00)

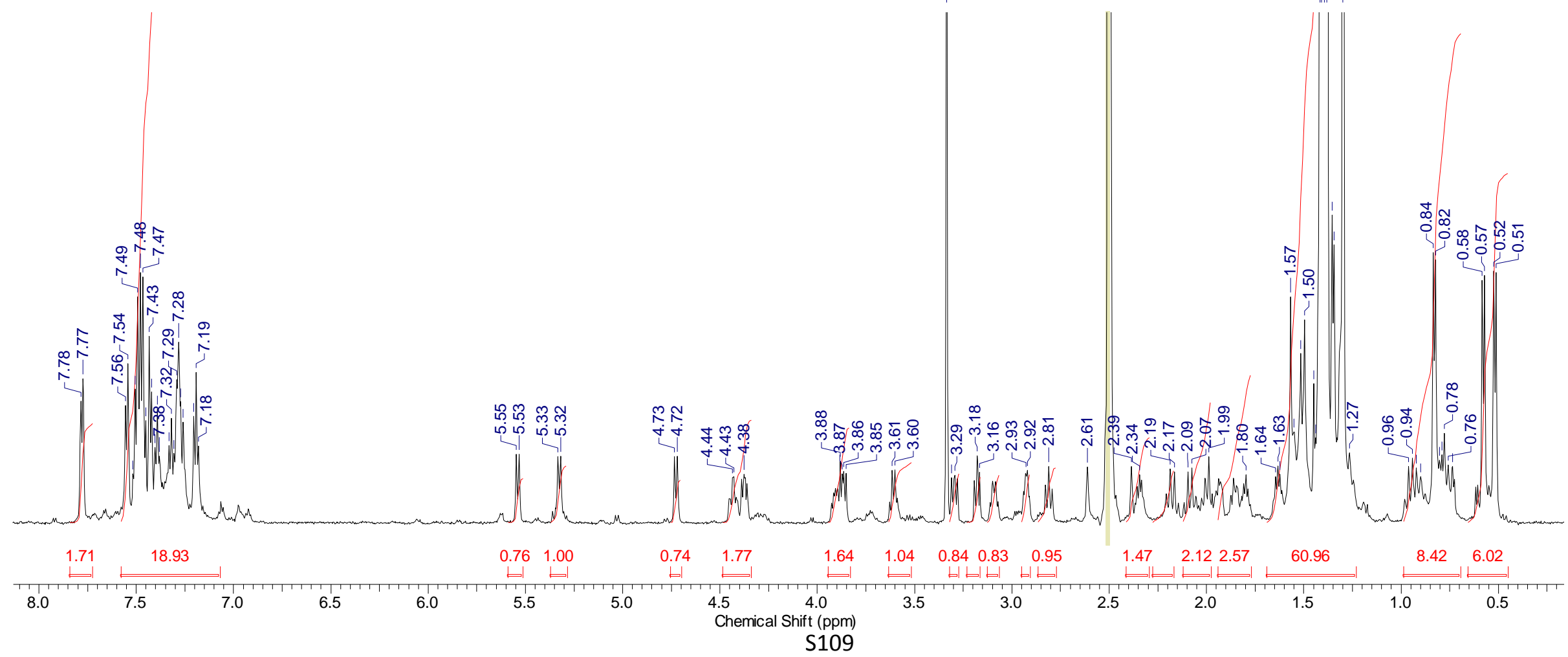



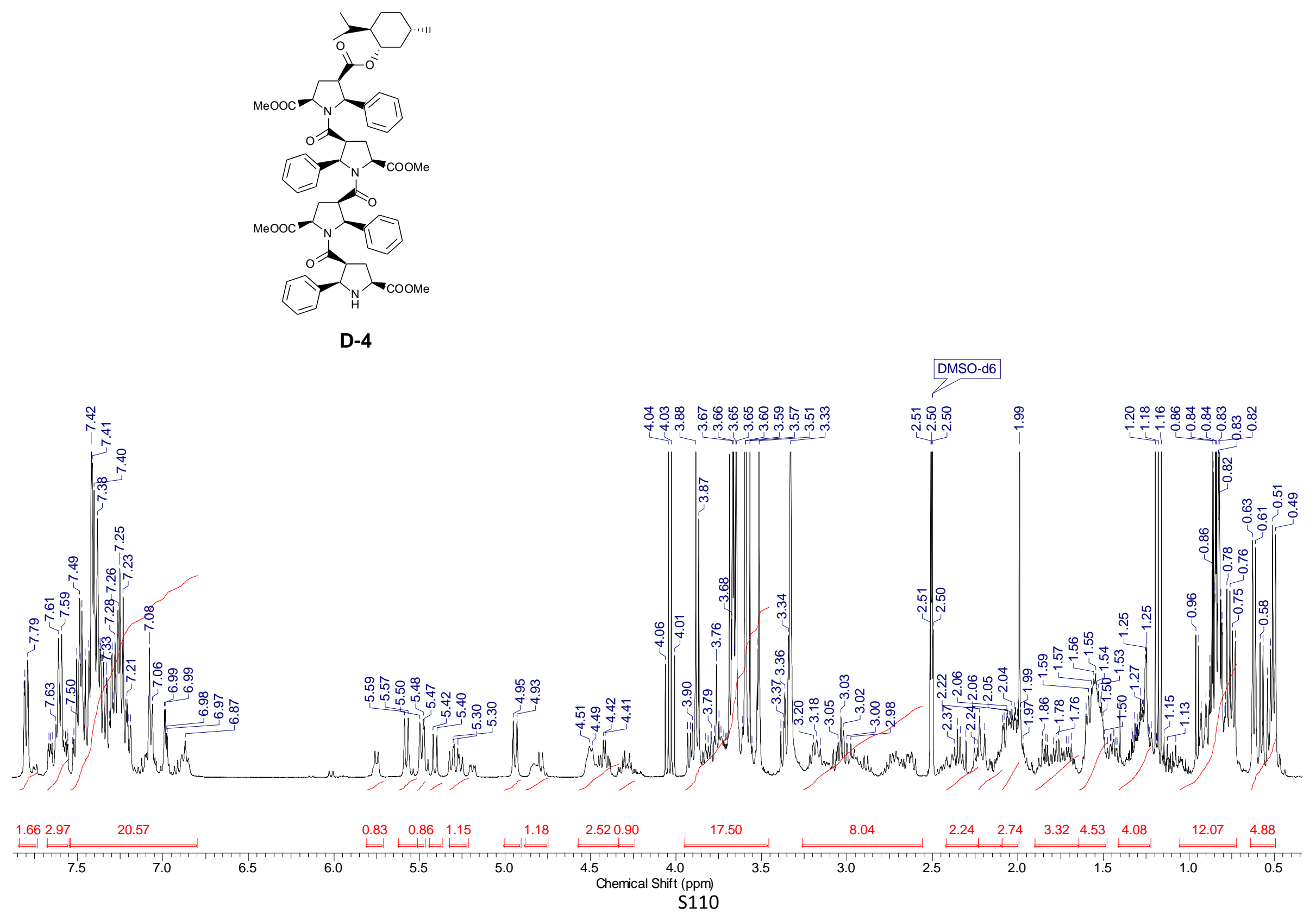

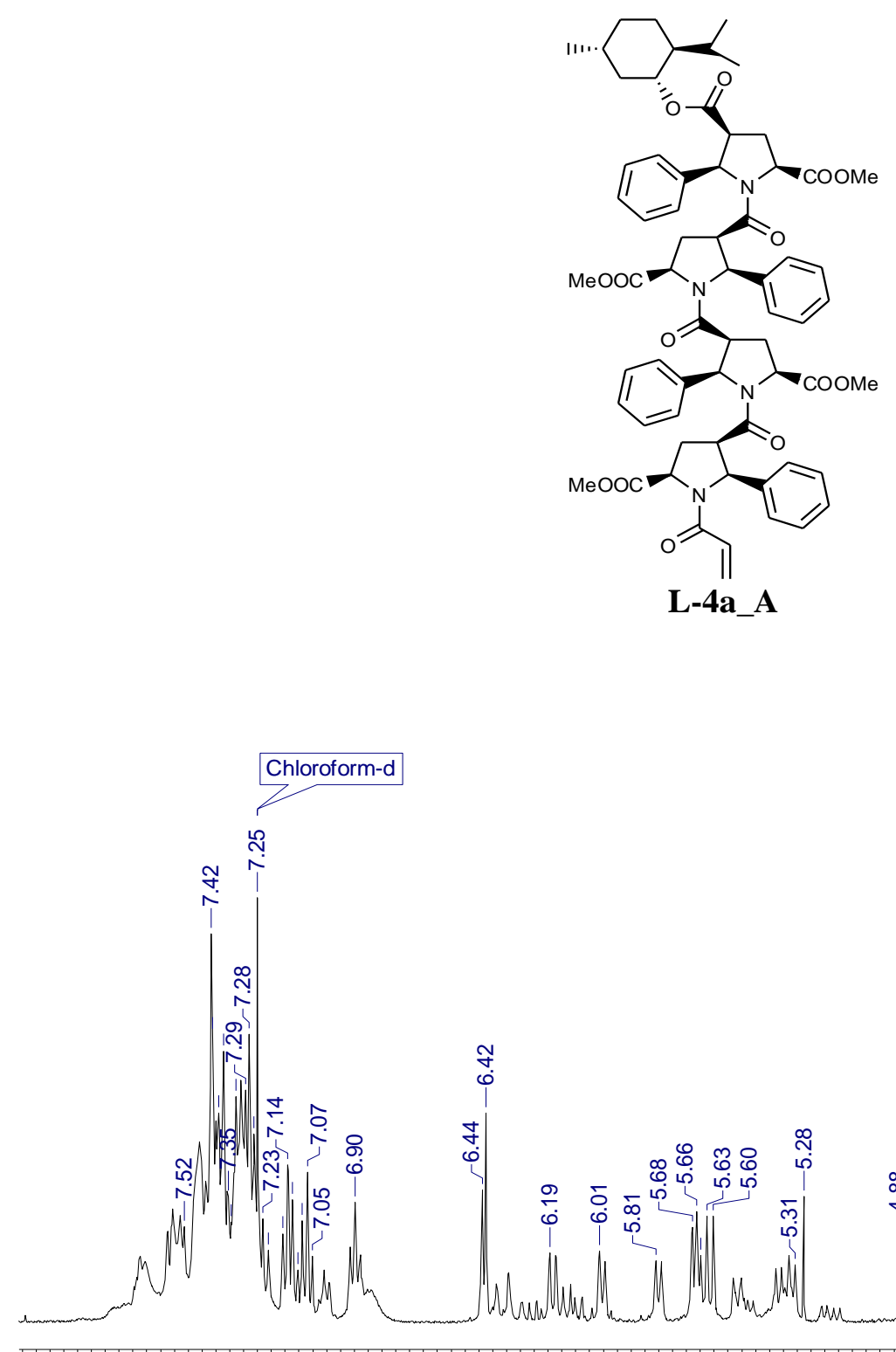

$1111+111111$
8.0

7.0

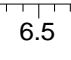




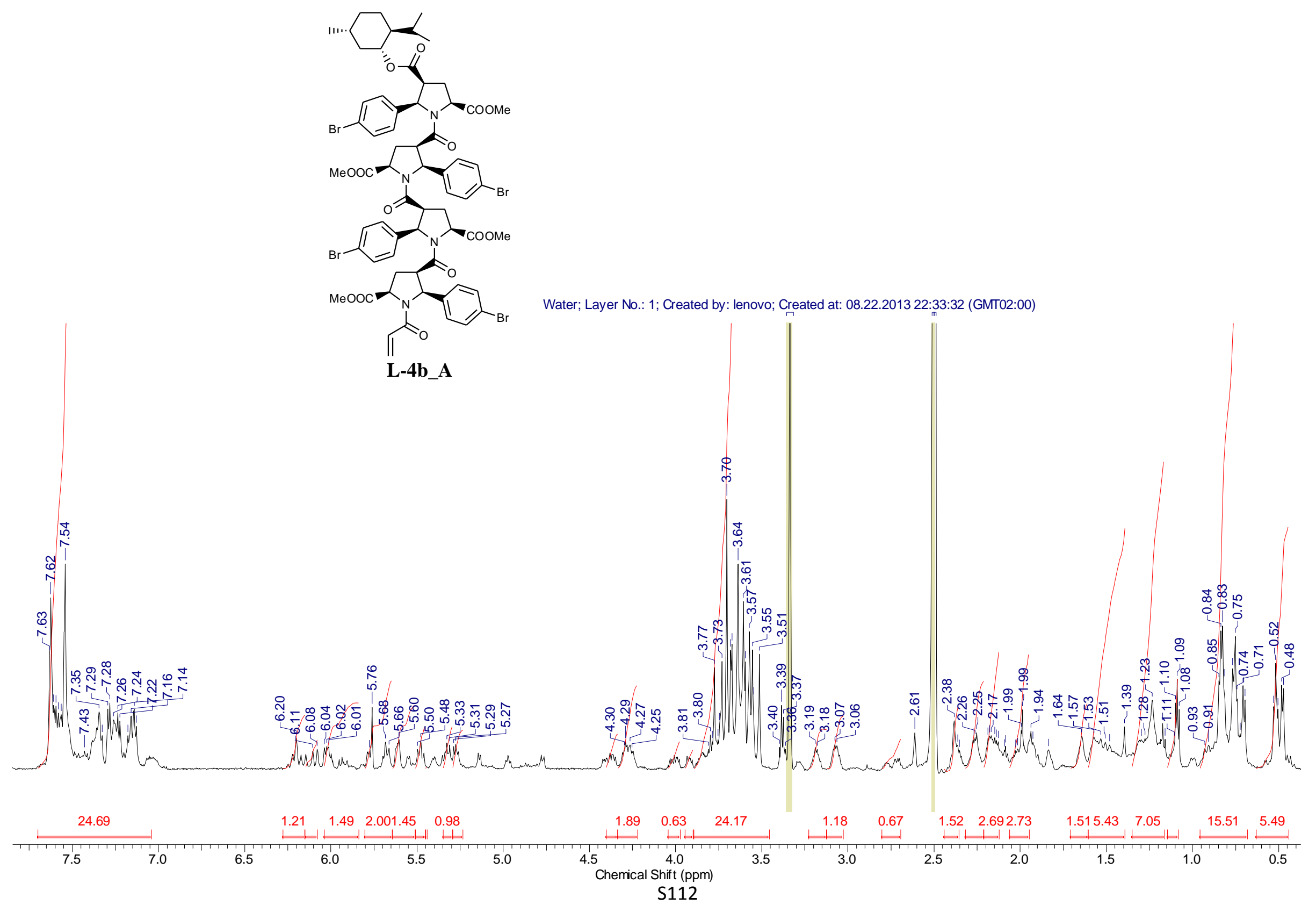




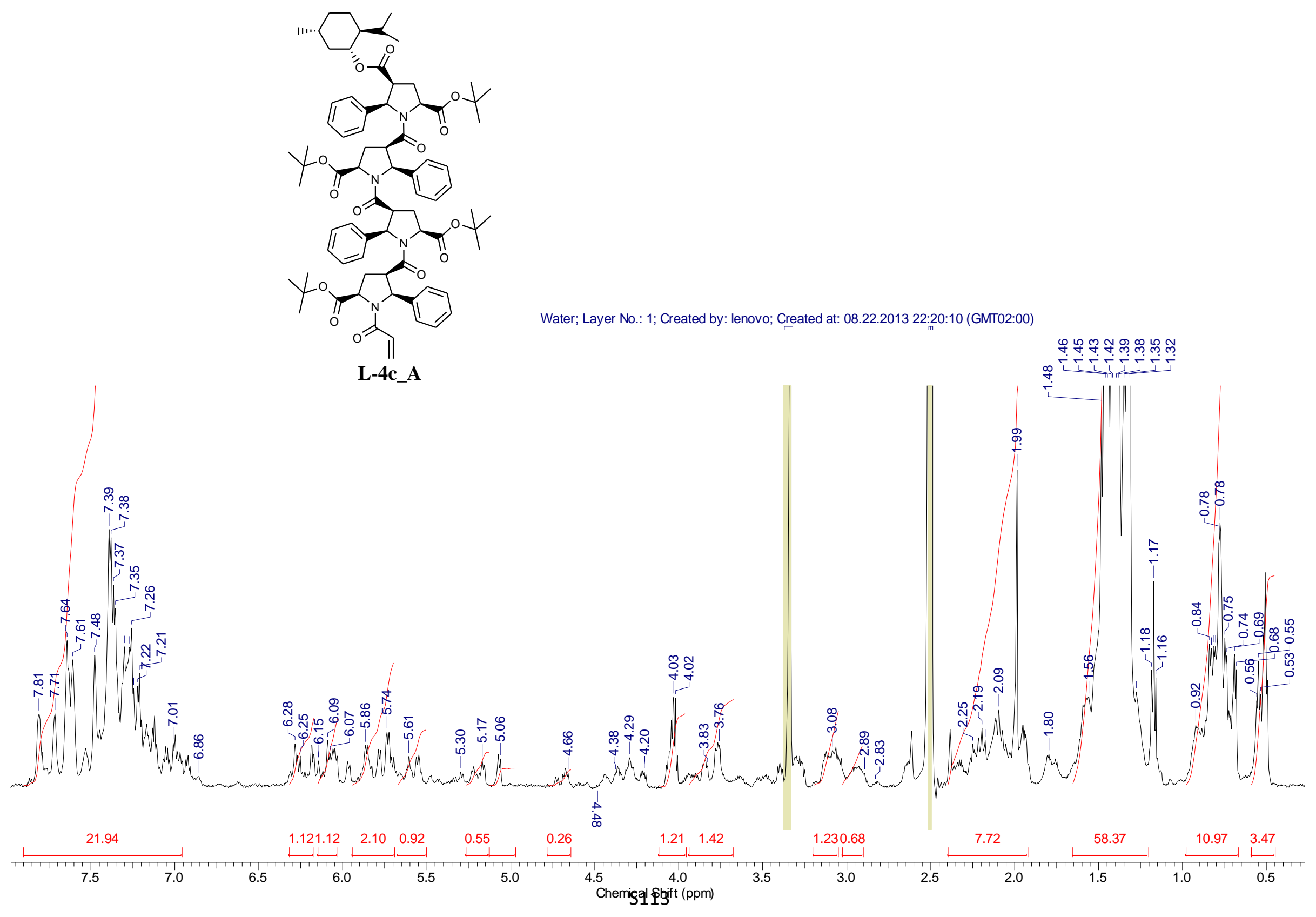




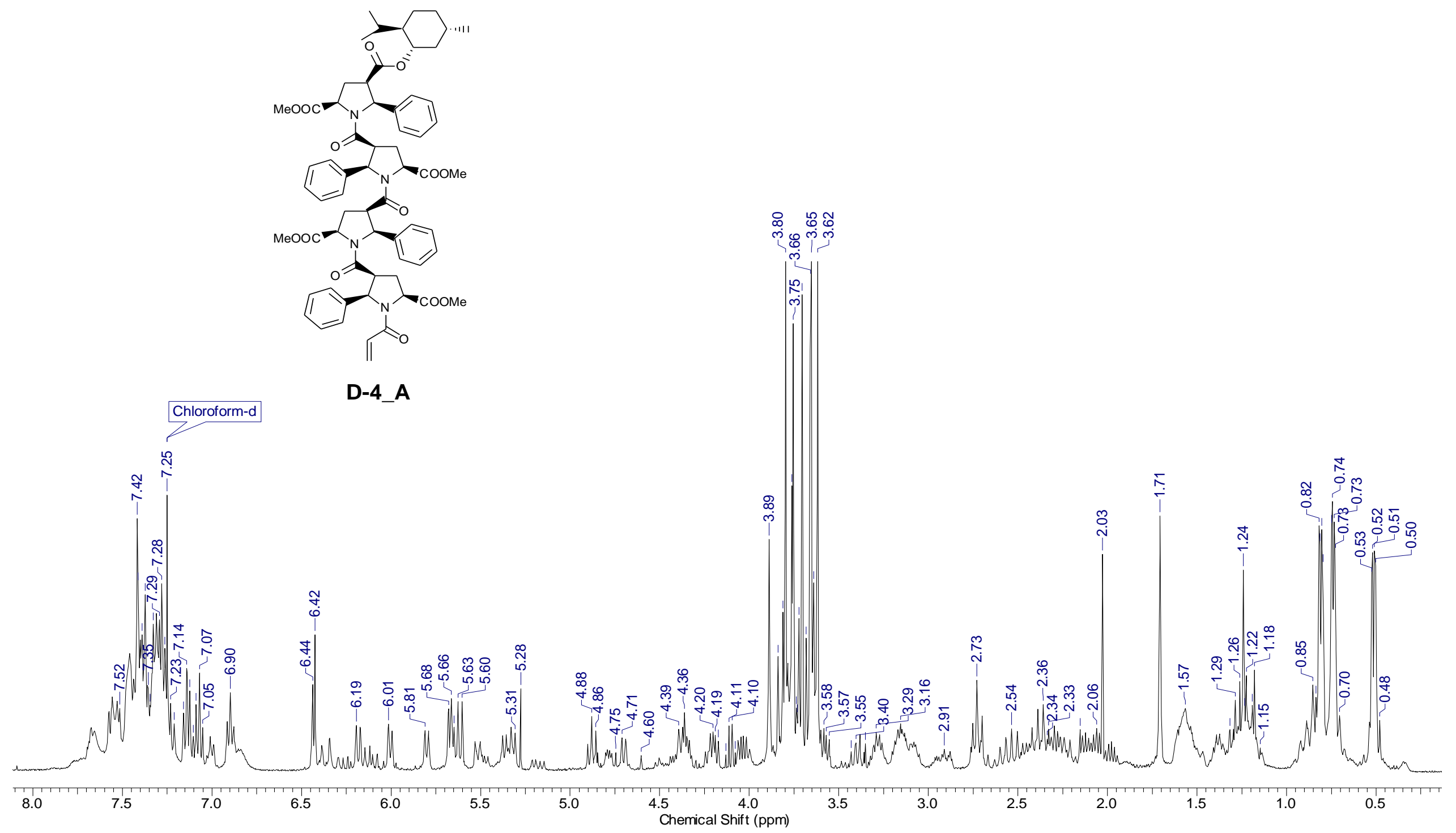




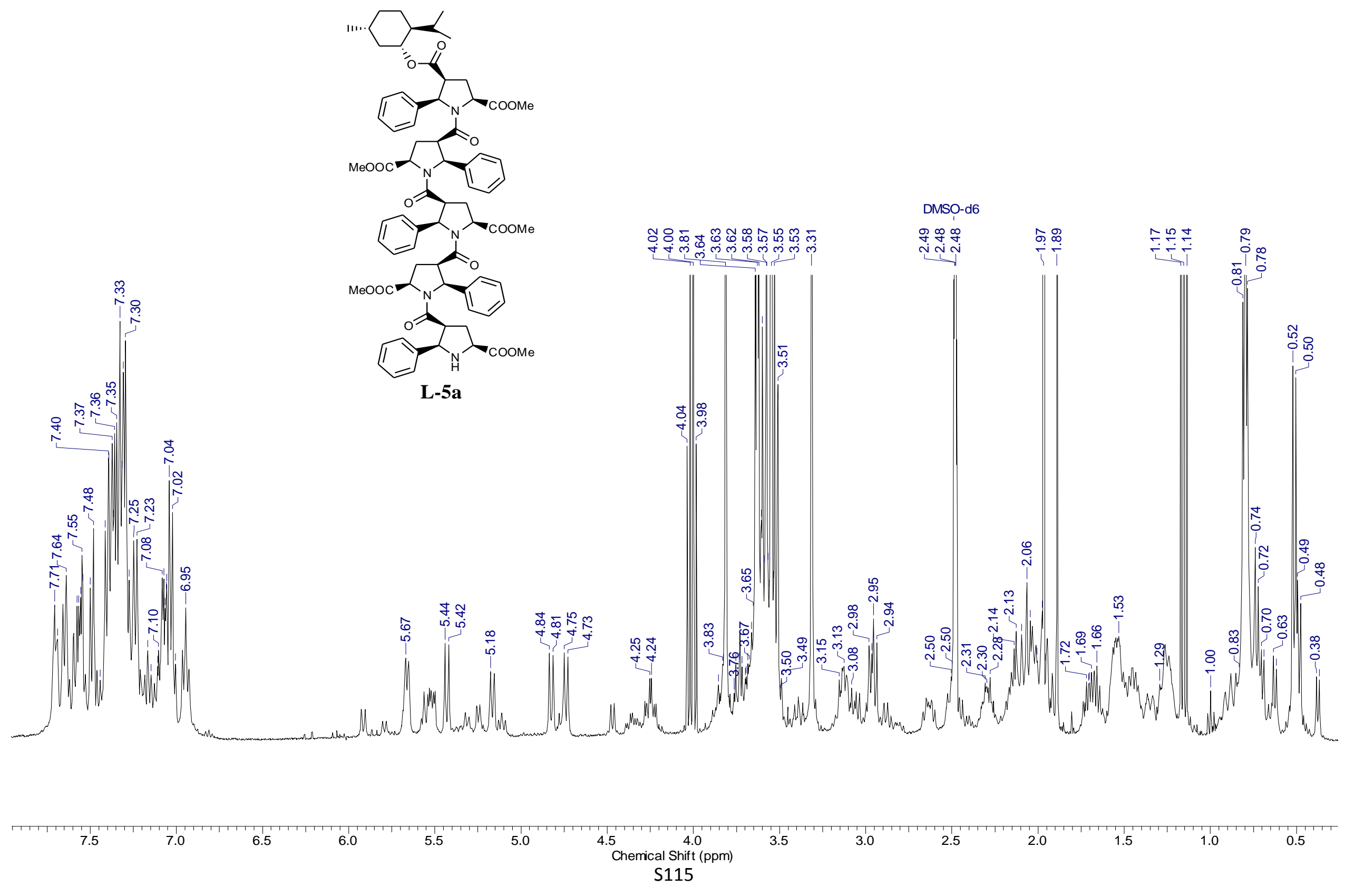




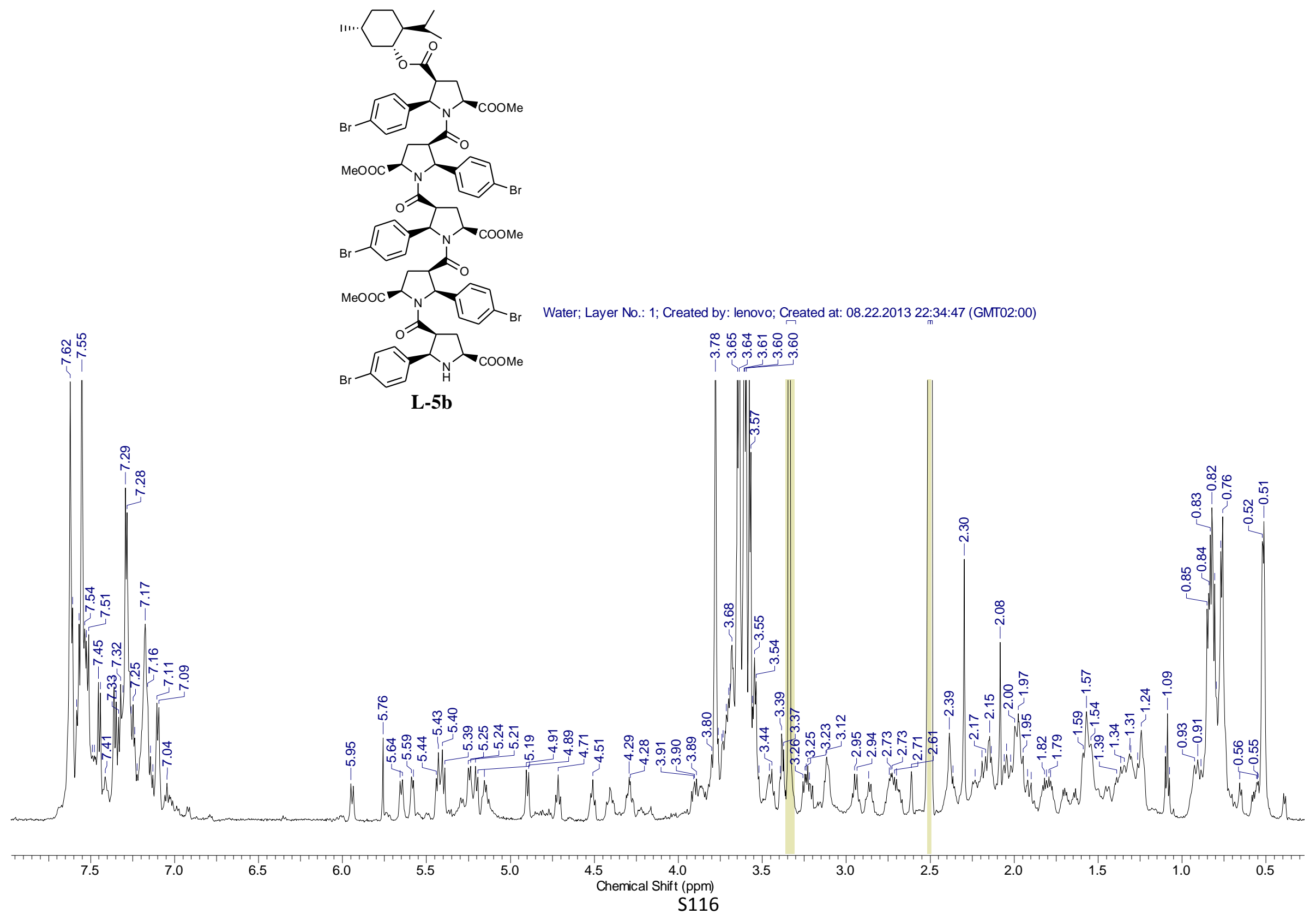




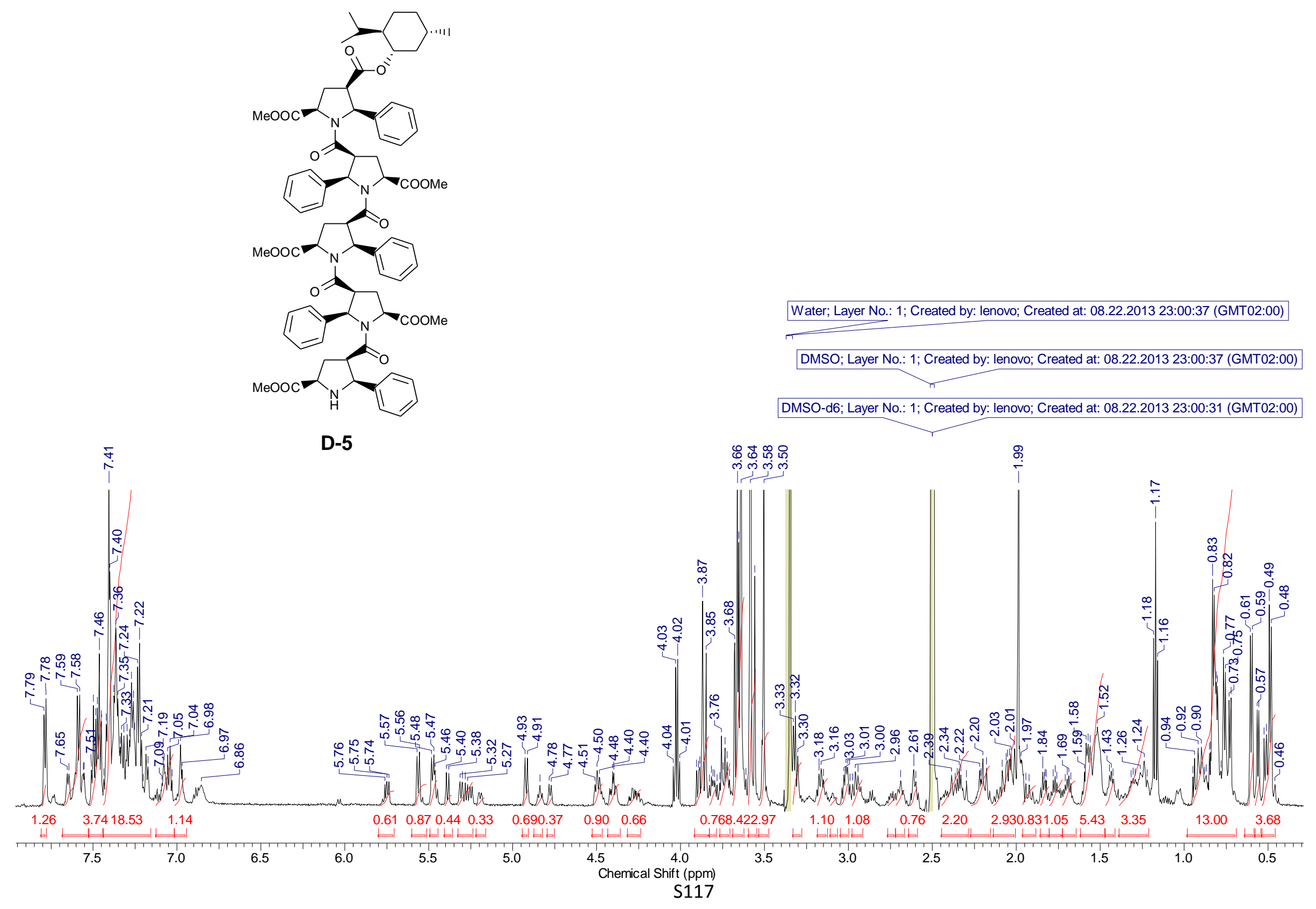




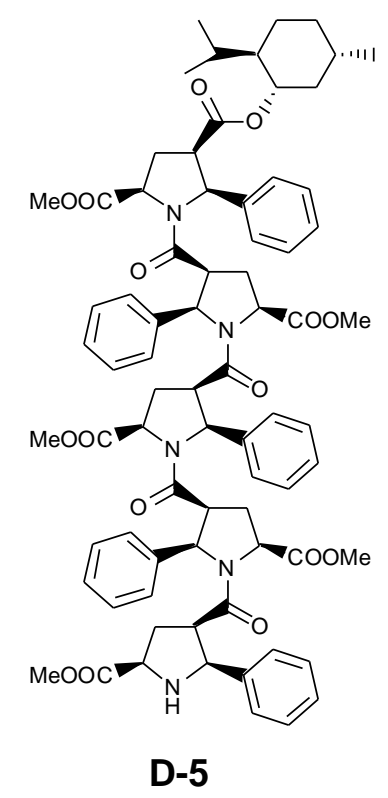

DMSO-d6; Layer No.: 1; Created by: lenovo; Created at: 08.22.2013 23:02:52 (GMT02:00)

둥요

구욤요욤

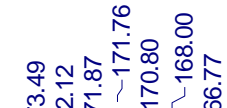

și

(1)

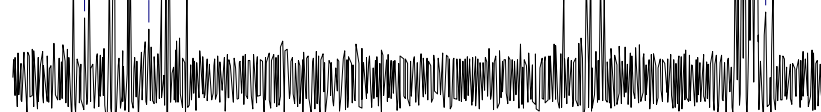

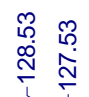

$176 \quad 168$

160

152

144

136

128

120

112

$104 \quad 96 \quad 88$
Chemical Shift (ppm)

80

48

40

32

24 


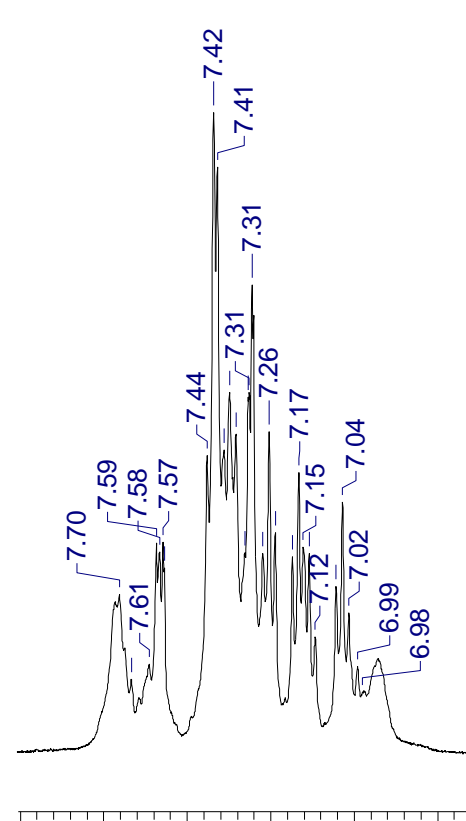

7.5

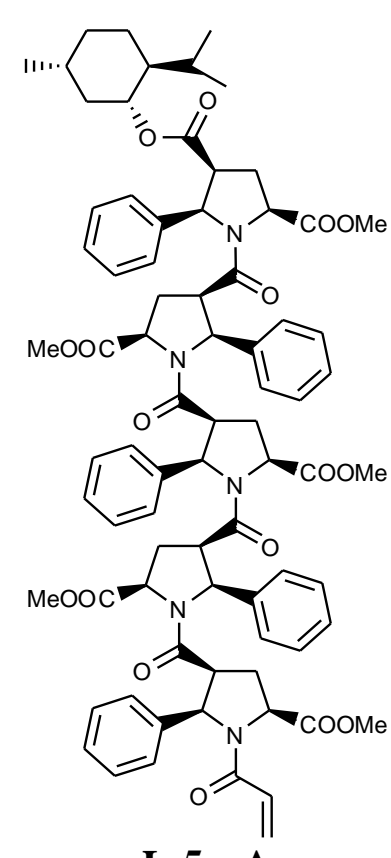

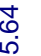

L-5a_A

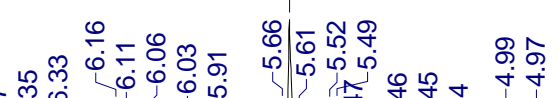
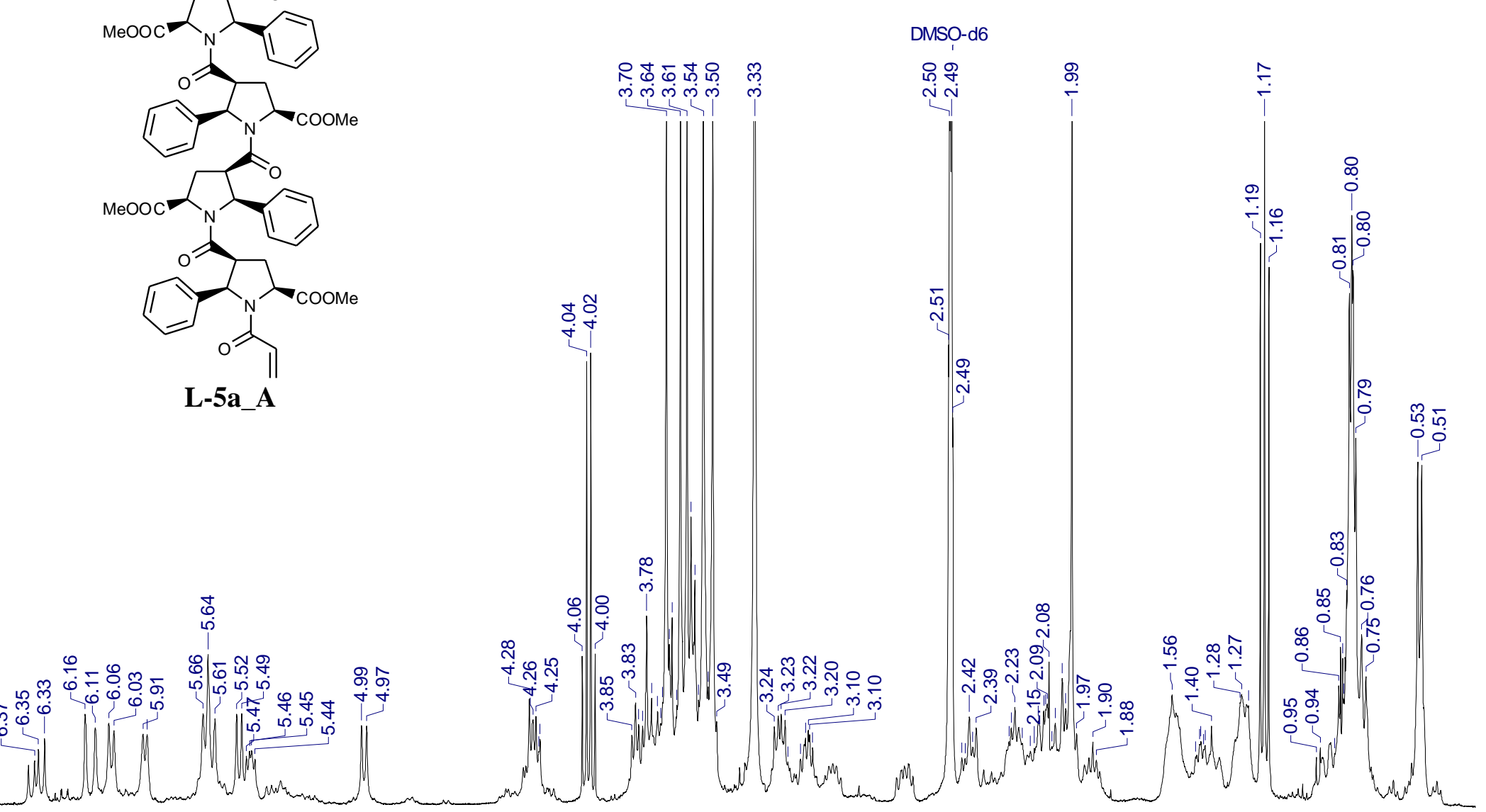

Chemical Shift (ppm)

3.5

3.0

2.5

2.0

1.5

$1.0 \quad 0.5$ 


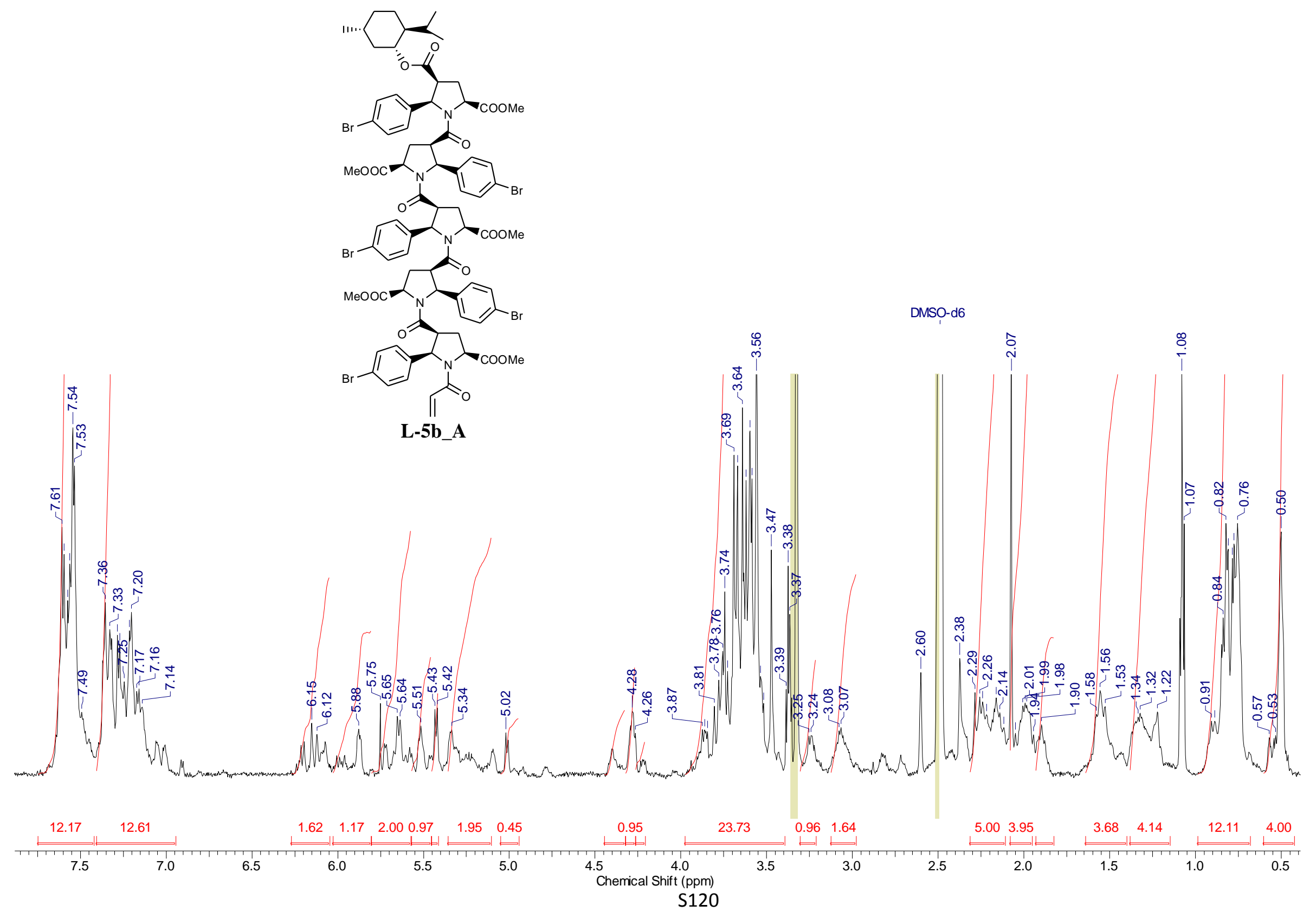




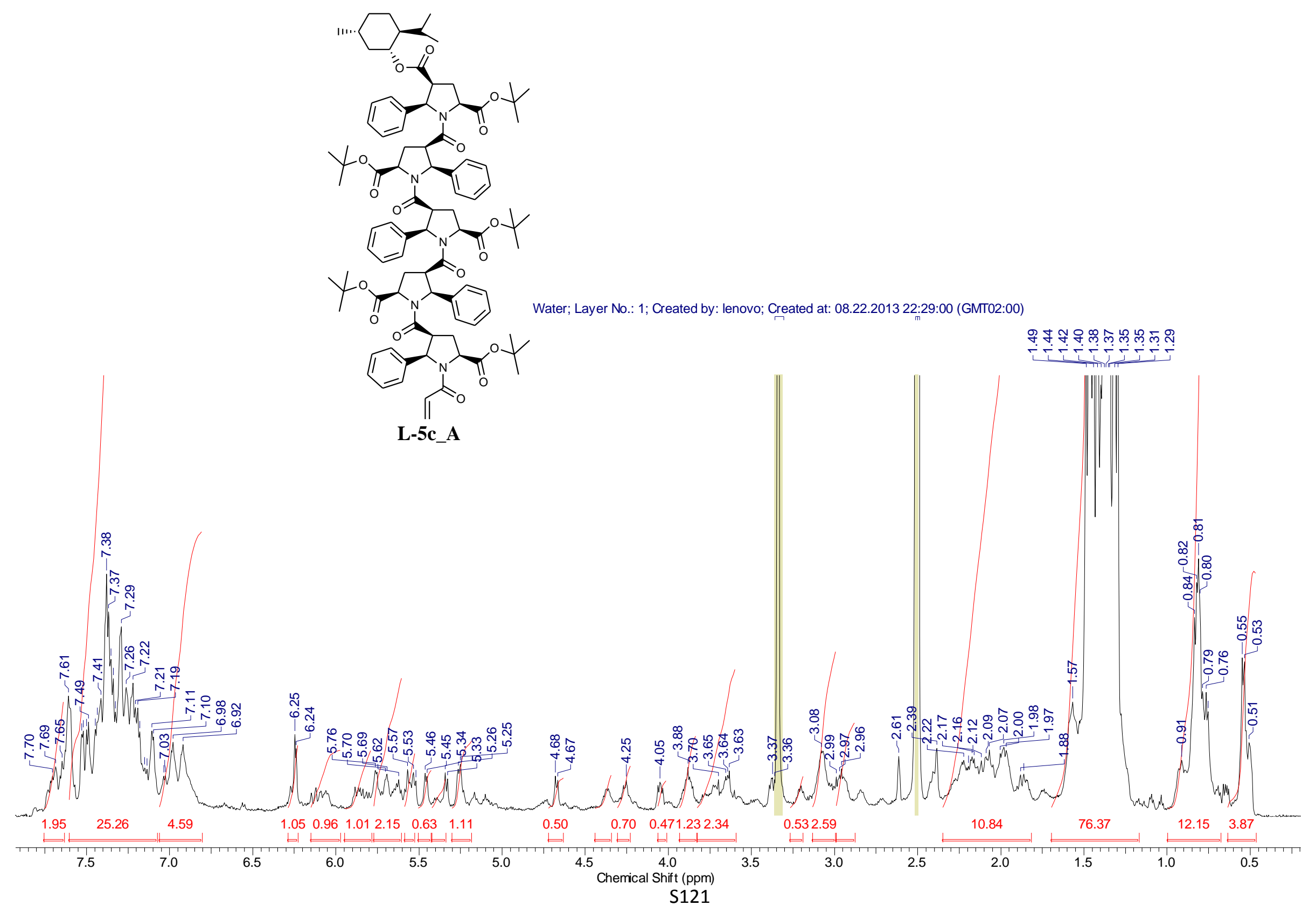




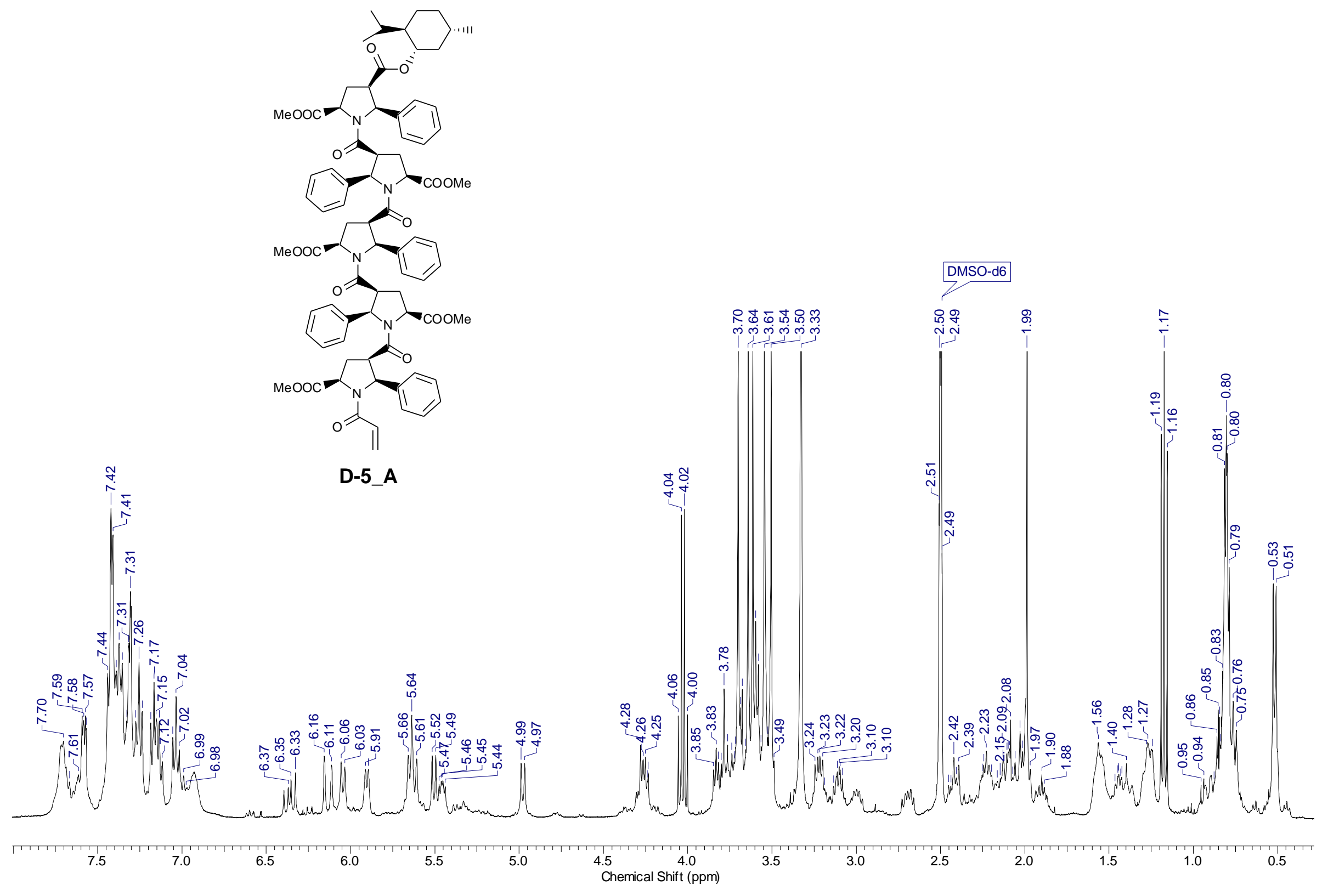

S122 


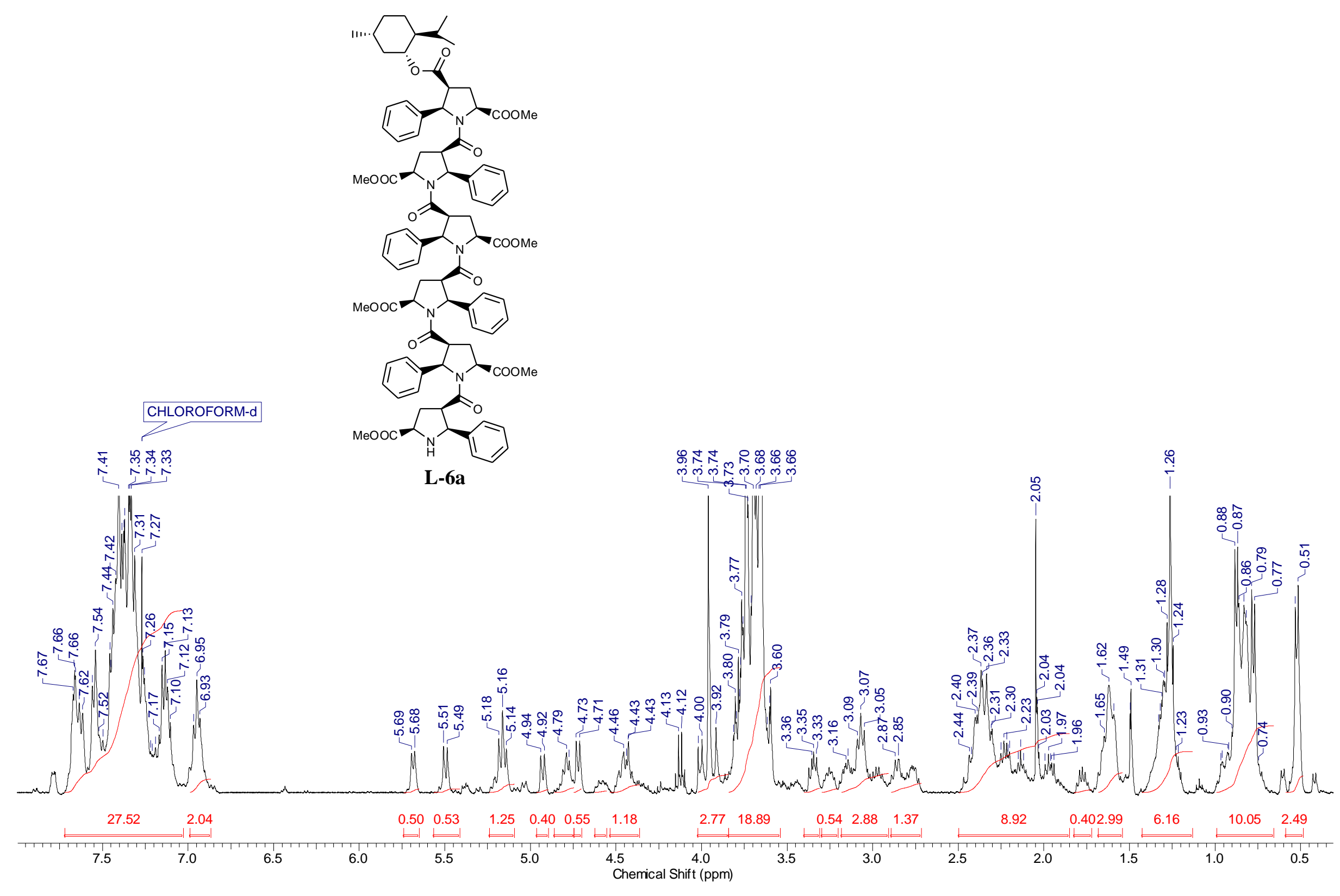

S123 


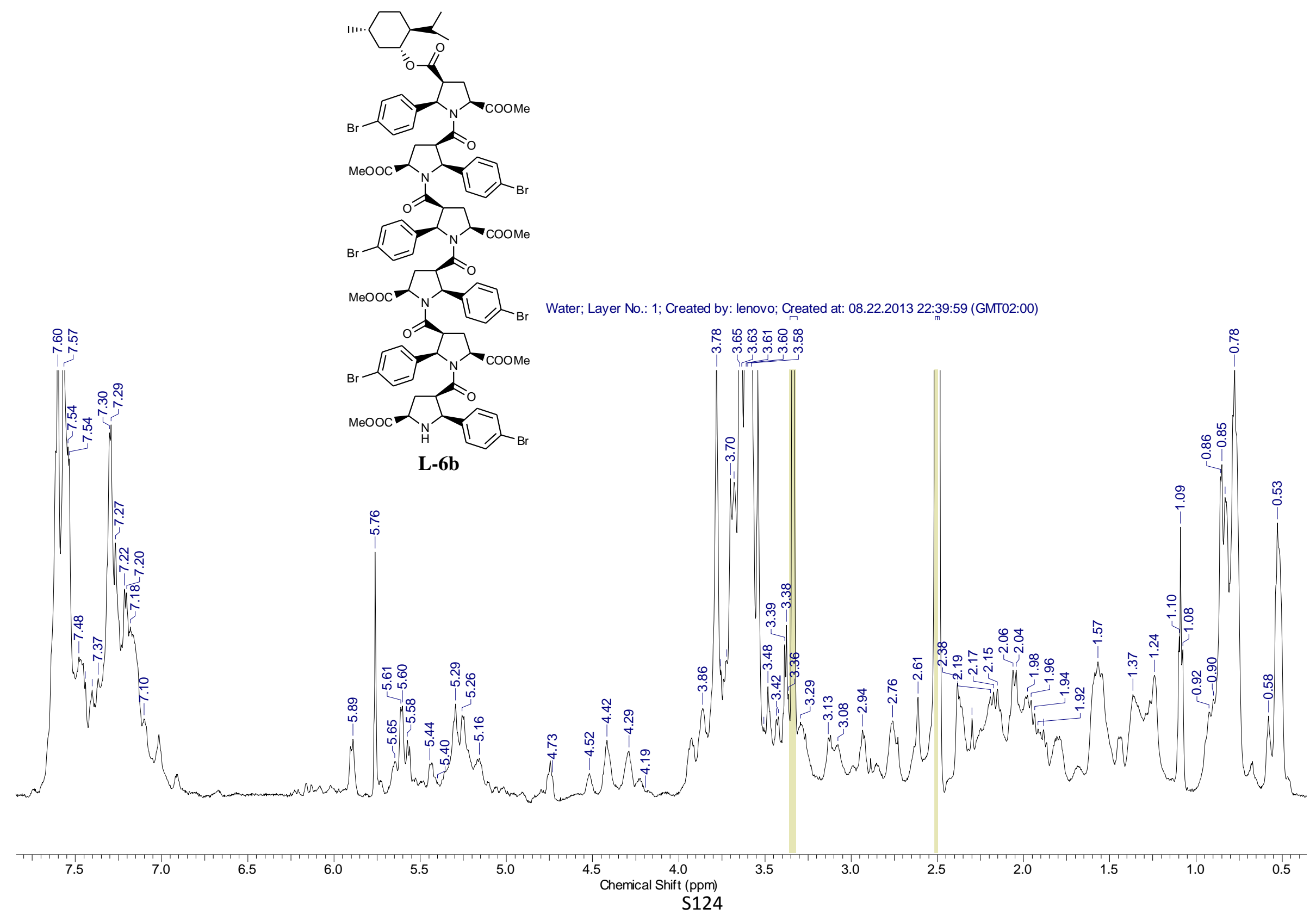




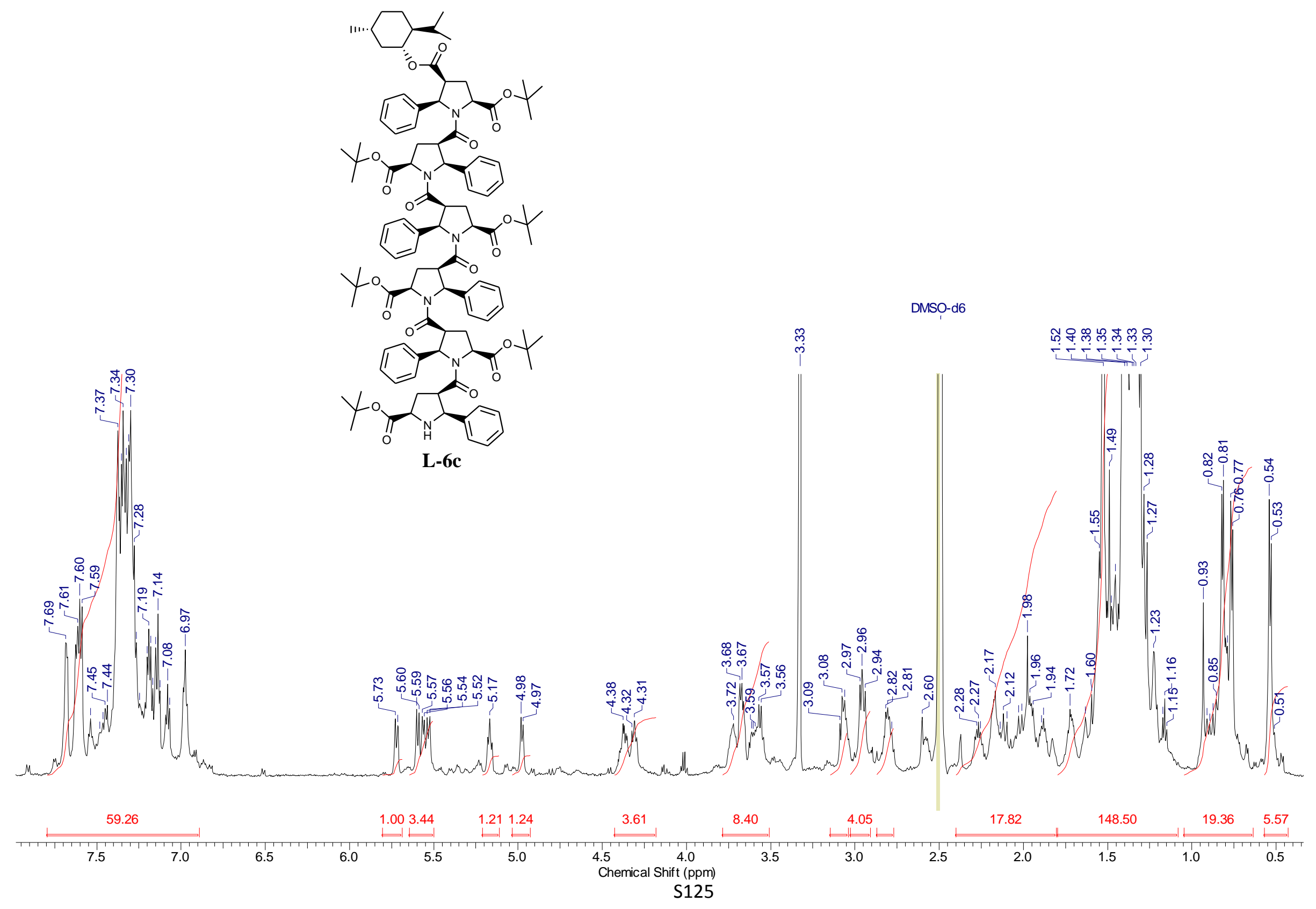




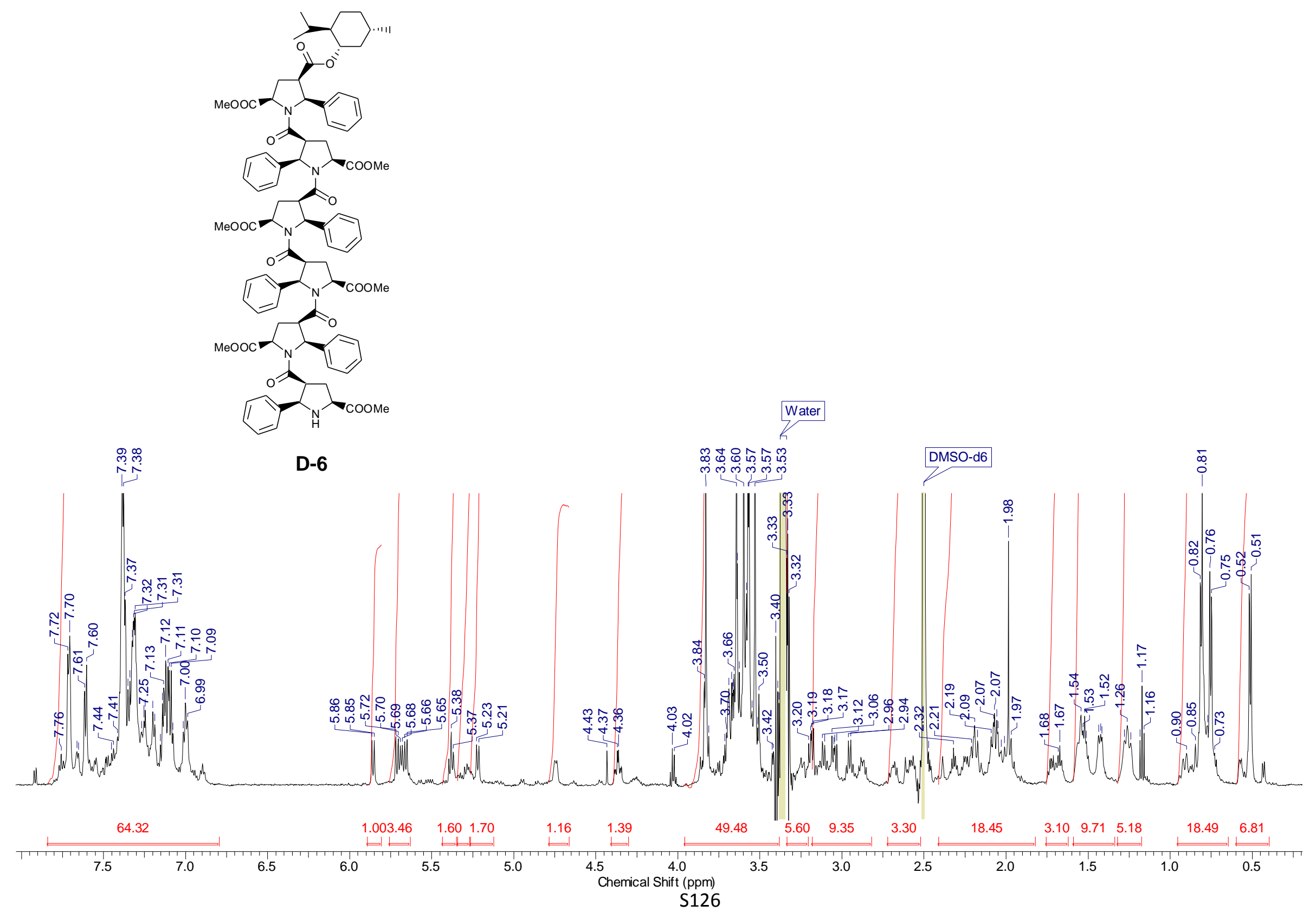

